\author{
Universidade de São Paulo \\ Instituto de Física
}

\title{
Aspectos Estruturais E DinÂMicos DA CORRESPONDÊNCIA ADS/CFT: UMA ABordagEM Rigorosa
}

\author{
Pedro Lauridsen Ribeiro
}

Orientador: João Carlos Alves Barata

Comissão Examinadora:

Prof. Dr. João Carlos Alves Barata (IF-USP)

Prof. Dr. Élcio Abdalla (IF-USP)

Prof. Dr. Frank Michael Forger (IME-USP)

Prof. Dr. Severino Toscano do Rêgo Melo (IME-USP)

Prof. Dr. Ricardo Schwarz Schör (ICEx-UFMG)

São Paulo 


\section{FICHA CATALOGRÁFICA}

Preparada pelo Serviço de Biblioteca e Informação do Instituto de Física da Universidade de São Paulo

Ribeiro, Pedro Lauridsen

Aspectos Estruturais e Dinâmicos da Correspondência AdS/CFT: uma

Abordagem Rigorosa. São Paulo, 2007.

Tese (Doutorado) - Universidade de São Paulo.

Instituto de Física - Departamento de

Física Matemática.

Orientador: Prof. Dr. João Carlos Alves Barata Área de Concentração: Física

Unitermos:

1. Física Matemática;

2. Teoria Quântica de Campo;

3. Relatividade (Física);

4. Geometria Semi-Riemanniana;

5. Álgebras de Operadores. 
Esta tese é dedicada à memória de minha avó, Maria de Lourdes Pimentel Pinto $\left({ }^{\star} 1922-{ }^{\dagger} 1996\right)$ e de minha tia-avó, Edith Pimentel Pinto ( ${ }^{\star} 1921$ - $\left.{ }^{\dagger} 1992\right)$, pois sei que olharam (e olham!) por mim esse tempo todo... 



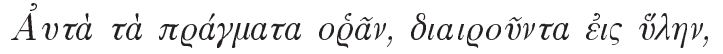

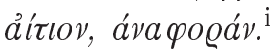

Marco Aurélio

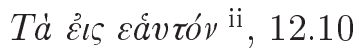

Man hört nur die Fragen, auf welche man imstande ist, eine Antwort zu finden. ${ }^{\mathrm{iii}}$

FriedRICH Wilhelm NietzSche Die fröhliche Wissenschaftiv ${ }^{\mathrm{iv}}, \S 196$ 



\section{Resumo.}

Elaboramos um estudo detalhado de alguns aspectos d(e uma versão d)a correspondência AdS/CFT, conjeturada por Maldacena [Mal98] e Witten [Wit98], entre teorias quânticas de campo num fundo gravitacional dado por um espaço-tempo assintoticamente anti-de Sitter (AAdS), e teorias quânticas de campos conformalmente covariantes no infinito conforme (no sentido de Penrose) deste espaço-tempo, aspectos estes: (a) independentes d(o par d)e modelos específicos em Teoria Quântica de Campos, e (b) suscetíveis a uma reformulação em moldes matematicamente rigorosos. Adotamos como ponto de partida o teorema demonstrado por Rehren [Reh00] no contexto da Física Quântica Local (também conhecida como Teoria Quântica de Campos Algébrica) em espaços-tempos anti-de Sitter (AdS), denominado holografia algébrica ou dualidade de Rehren.

O corpo do presente trabalho consiste em estender o resultado de Rehren para uma classe razoavelmente geral de espaços-tempos AAdS $d$-dimensionais $(d>3)$, escrutinar como as propriedades desta extensão são enfraquecidas e/ou modificadas em relação ao espaço-tempo AdS, e como efeitos gravitacionais não-triviais se manifestam na teoria quântica no infinito conforme.

Dentre os resultados obtidos, citamos: condições razoavelmente gerais sobre geodésicas nulas no interior (cuja plausibilidade justificamos por meio de resultados de rigidez geométrica) não só garantem que a nossa generalização é geometricamente consistente com causalidade, como também permite uma reconstrução "holográfica" da topologia do interior na ausência de horizontes e singularidades; a implementação das simetrias conformes na fronteira, que associamos explicitamente a uma família de isometrias assintóticas do interior construída de maneira intrínseca, ocorre num caráter puramente assintótico e é atingida dinamicamente por um processo de retorno ao equilíbrio, mediante condições de contorno adequadas no infinito; efeitos gravitacionais podem eventualmente causar obstruções à reconstrução da teoria quântica no interior, ou por torná-la trivial em regiões suficientemente pequenas ou devido à existência de múltiplos vácuos inequivalentes, que por sua vez levam à existência de excitações solitônicas localizadas ao redor de paredes de domínio no interior, similares a D-branas. As demonstrações fazem uso extensivo de geometria Lorentziana global. A linguagem empregada para as teorias quânticas relevantes para nossa generalização da dualidade de Rehren segue a formulação funtorial de Brunetti, Fredenhagen e Verch para a Física Quântica Local [BFV03], estendida posteriormente por Sommer [Som06] para incorporar condições de contorno. 



\begin{abstract}
.
We elaborate a detailed study of certain aspects of (a version of) the AdS/CFT correspondence, conjectured by Maldacena [Mal98] and Witten [Wit98], between quantum field theories in a gravitational background given by an asymptotically anti-de Sitter (AAdS) spacetime, and conformally covariant quantum field theories in the latter's conformal infinity (in the sense of Penrose), aspects such that: (a) are independent from (the pair of) specific models in Quantum Field Theory, and (b) susceptible to a recast in a mathematically rigorous mould. We adopt as a starting point the theorem demonstrated by Rehren [Reh00] in the context of Local Quantum Physics (also known as Algebraic Quantum Field Theory) in anti-de Sitter (AdS) spacetimes, called algebraic holography or Rehren duality.

The main body of the present work consists in extending Rehren's result to a reasonably general class of $d$-dimensional AAdS spacetimes $(d>3)$, scrutinizing how the properties of such an extension are weakened and/or modified as compared to AdS spacetime, and probing how non-trivial gravitational effects manifest themselves in the conformal infinity's quantum theory.

Among the obtained results, we quote: not only does the imposition of reasonably general conditions on bulk null geodesics (whose plausibility we justify through geometrical rigidity techniques) guarantee that our generalization is geometrically consistent with causality, but it also allows a "holographic" reconstruction of the bulk topology in the absence of horizons and singularities; the implementation of conformal symmetries in the boundary, which we explicitly associate to an intrinsically constructed family of bulk asymptotic isometries, have a purely asymptotic character and is dynamically attained through a process of return to equilibrium, given suitable boundary conditions at infinity; gravitational effects may cause obstructions to the reconstruction of the bulk quantum theory, either by making the latter trivial in sufficiently small regions or due to the existence of multiple inequivalent vacua, which on their turn lead to the existence of solitonic excitations localized around domain walls, similar to D-branes. The proofs make extensive use of global Lorentzian geometry. The language employed for the quantum theories relevant for our generalization of Rehren duality follows the functorial formulation of Local Quantum Physics due to Brunetti, Fredenhagen and Verch [BFV03], extended afterwards by Sommer [Som06] in order to incorporate boundary conditions.
\end{abstract}





\section{Agradecimentos}

Meu sincero obrigado para o Prof. João Barata, por me acolher no Departamento de Física Matemática, por ter-me proposto o presente tema de investigação e por ter acreditado em mim mesmo nos momentos mais difíceis do desenvolvimento deste projeto, dando assim suporte sólido e essencial.

A meu antigo grupo de iniciação científica, amigos até hoje que muito estimo: Profs. Alinka Lépine-Szily, Rubens Lichtenthäler Filho e Valdir Guimarães, pelo apoio e incentivo quando decidi migrar da Física Nuclear experimental/computacional para a Física Matemática, e sobretudo por me ensinarem o bê-a-bá da pesquisa científica e uma atitude íntegra frente a ela.

Aos (ex-)colegas do Departamento de Física Matemática (IFUSP) Alessandro Marques, Alex Dias, Amílcar Queiroz, Brenno Vallilo, Davi Giugno, Ivan Pontual, Karlucio Castello Branco, Leandro Beviláqua, Marcelo Pires, "digníssimo" Leonardo Sioufi e Patrícia Teles, pelas conversas impagáveis (em ambos os sentidos!) e pela companhia ao longo desses mais que quatro anos.

Aos mestres e companheiros do Instituto de Matemática e Estatística, por terem sido abertos às enriquecedoras trocas de idéias que muito contribuíram para meu aprendizado, em particular o grupo de Álgebras de Operadores (Cíntia da Silva, David Dias, Marcela Merklen, Patrícia Hess e Profs. Cristina Cerri, Martha Salerno, Ricardo Bianconi e Severino Toscano), o grupo do Prof. Michael Forger (Bruno Soares, Eder Annibale, Fernando Antoneli, Getúlio Bulhões, Leandro Gomes, Mário Salles, Paola Gaviria, Sandra Yepes e Sandro Romero) e também aos Profs. Helena Ávila, Paulo Cordaro e aos colegas Alexandre Kawano, Arthur Julião, Antônio Ronaldo Garcia e Nestor Centurión.

Gostaria também de agradecer aos Profs. Bert Schroer, Christian Jäkel, Daniel Vanzella, Fernando Auil, Jens Mund e, uma vez mais, ao Prof. Michael Forger pela paciência e entusiasmo durante sua participação na série de seminários informais ao longo dos anos 2004-2005 dedicada ao estudo da referência [BFV03], que constitui pe- 
dra fundadora do arcabouço conceitual subjacente à presente Tese. Agradeço outrossim a estes e aos Profs. Aiyalam Balachandran, Detlev Buchholz, Henning Rehren, Klaus Fredenhagen, Kostas Skenderis, Sumati Surya e Walter Wreszinski, pelos diálogos e/ou sequências de troca de emails inspiradores em momentos cruciais do desenvolvimento do presente trabalho, e que, assim, constituíram auxílio inestimável com suas sugestões e críticas construtivas.

Às secretárias do Departamento de Física Matemática: Amélia Genova, Elizabeth Vargas e Simone Shinomiya, pela solicitude e incansável simpatia.

À FAPESP, pelo apoio financeiro (este projeto foi agraciado com Bolsa de Doutorado Direto referente ao processo 01/14360-1).

Bom, é bastante gente, e certamente esqueci alguém acima... Obrigado e desculpas a estes!

E, por último (e primeiro), os agradecimentos mais que especiais:

A meus pais, pois esta jornada também foi deles, de todos nós!

Ao meu irmão Francisco, pelo contraponto espiritual.

À Clau, por manter (aturar!) a minha (in)sanidade mental, e me ensinar a importância das coisas que precisam ser ditas.

A Deus, Pai Eterno Todo-Poderoso, por me mostrar um caminho que me fascinou e me dar forças para segui-lo. Oxalá renda bons frutos em Sua honra! 


\section{Sumário}

Prefácio $\quad$ xvii

$\begin{array}{ll}\text { Lista de Figuras } & \text { xxv }\end{array}$

Notações e convenções de uso freqüente $\quad$ xxvii

I A correspondência AdS/CFT e suas manifestações geométricas

1 A geometria dos espaços-tempos assintoticamente anti-DE SITTER 3

1.1 Generalidades sobre formas espaciais . . . . . . . . . . . . 3

1.2 Geometria de $A d S_{d}$ : condições de contorno no infinito . . . . . . . . . 6

1.2.1 Estrutura do infinito conforme . . . . . . . . . . . . . 7

1.2.2 Cunhas em AdS e diamantes na fronteira . . . . . . . . . . . . 9

1.3 Espaços-tempos AAdS . . . . . . . . . . . . . . . . . 14

1.3.1 Geometria global e geodésicas nulas . . . . . . . . . . . . 16

1.3.1.1 Causalidade (interior para fronteira) . . . . . . . 16

1.3.1.2 Conexidade simples (interior versus fronteira) . . . . 23

1.3.1.3 Localização (fronteira para interior) . . . . . . . . 24

2 Dinâmica e evolução temporal $\quad 31$

2.1 Gravitação . . . . . . . . . . . . . . . . . . . . . 31

2.2 A expansão de Fefferman-Graham . . . . . . . . . . . . . . . 33

2.3 "Termodinâmica" gravitacional em cunhas AAdS . . . . . . . . . . . . 40

2.3.1 Evolução temporal geométrica em diamantes . . . . . . . . . . . . 40

2.3.2 Construção de isometrias assintóticas . . . . . . . . . . . . . 47

2.3.3 "Retorno ao equilíbrio" em cunhas . . . . . . . . . . . . . 51 


\section{Avatares holográficos da Física Quântica Local}

3 Teorias Quânticas Localmente Covariantes $\quad 59$

3.1 Definições básicas . . . . . . . . . . . . . . . . . . . . 60

3.2 Relação com os axiomas usuais . . . . . . . . . . . . . . . . 61

3.3 Estados quânticos em espaços-tempos curvos . . . . . . . . . . . 64

3.4 Campos quânticos localmente covariantes . . . . . . . . . . . . 64

4 Implementando o Princípio Holográfico em espaços-tempos AAdS 69

4.1 Covariância local com condições de contorno . . . . . . . . . . . 69

4.2 Teorias quânticas localmente covariantes em AdS . . . . . . . . . . . . 74

4.2.1 Valores de fronteira como condição dinâmica . . . . . . . . 75

4.3 Holografia algébrica em espaços-tempos AAdS . . . . . . . . . . . 84

4.4 Análise holográfica dos setores de superseleção . . . . . . . . . . . . 87

4.4 .1 Uma digressão . . . . . . . . . . . . . . . . . . . . . . 88

4.4.2 Dualidade de HAAg (essencial) em espaços-tempos (A)AdS . . 91

4.4.3 Obstruções à inversão da dualidade de REHREn . . . . . . . . 97

$\begin{array}{ll}\text { III Uma síntese } & 99\end{array}$

$\begin{array}{ll}\text { Coda } & 101\end{array}$

$\begin{array}{ll}\text { Apêndices } & 104\end{array}$

A Elementos de geometria Lorentziana 107

A.1 Teoria local . . . . . . . . . . . . . . . . . . . . . . 107

A.2 Teoria global . . . . . . . . . . . . . . . . . . . . 113

A.2.1 Estrutura causal, completeza geodésica . . . . . . . . . 113

A.2.2 Infinito conforme . . . . . . . . . . . . . . 119

B Elementos de álgebras de operadores 123

B.1 *-Álgebras . . . . . . . . . . . . . . . . . . . . . . . 124

B.2 $\mathrm{C}^{*}$-álgebras e álgebras de von Neumann $\ldots \ldots \ldots \ldots \ldots$

B.2.1 C*álgebras . . . . . . . . . . . . . . . . . . . 129

B.2.2 Álgebras de von Neumann . . . . . . . . . . . . . . . . . 132

B.2.3 Teoria modular de Tomita-TAKESAKi . . . . . . . . . . 136

B.2.4 Sistemas $\mathrm{C}^{*}$ - e $\mathrm{W}^{*}$-dinâmicos. Condição KMS . . . . . . . . 140

B.3 *álgebras localmente convexas e álgebras de Borchers-Uhlmann . 144 
$\begin{array}{llr}\text { C Categorias e funtores } & 149\end{array}$

C.1 Rudimentos . . . . . . . . . . . . . . . . . . . . . . . . 149

C.2 Funtores e transformações naturais . . . . . . . . . . . . . . . 152

D Rudimentos de homotopia $\quad 157$

D.1 Aplicações homotópicas . . . . . . . . . . . . . . . . . . . . . . . 157

D.2 O grupo fundamental e espaços de recobrimento . . . . . . . . . . 158

$\begin{array}{ll}\text { Referências Bibliográficas } & 161\end{array}$

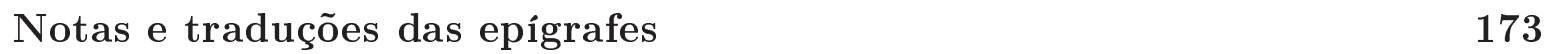





\section{Prefácio}

\section{SemÂnTicA}

Eu sei lá o que querem dizer as palavras.

Sou caminho

e se imanto as limalhas a meu jeito

o desenho não é figurativo.

E se empreendo, gaguejando,

a leitura do meu campo de esforço

o feito

infante ainda

é mensagem cifrada.

Edith Pimentel Pinto

"Normativa" (Sinais e Conhecenças ${ }^{\mathrm{v}}$, 1986)

Dois dos maiores problemas em aberto da Física Teórica contemporânea são a falta de compreensão quantitativa dos aspectos não-perturbativos das Teorias de Gauge nãoAbelianas (confinamento de quarks, etc.) e a quantização do campo gravitacional, que se faz fenomenologicamente necessária devido aos limites de consistência física da Relatividade Geral, que descreve a gravitação clássica até onde fomos capazes de testar experimentalmente. Uma das idéias correntes mais tantalizantes na literatura é a de que ambos os problemas estão, de certa forma, ligados por uma correspondência holográfica, na qual a gravitação numa certa região do espaço-tempo admitiria uma descrição "dual" na fronteira desta região por meio de uma teoria de gauge, da mesma forma que um holograma bidimensional reproduz uma imagem tridimensional. Mais ainda, os regimes de energia dos processos físicos associados às duas teorias são de natureza tal que aspectos não-perturbativos da teoria de gauge se manifestariam no regime gravitacional (semi-)clássico. 
A história de como essa idéia surgiu remonta à década de 70. As 4 leis da dinâmica de buracos negros, estabelecidas por BARDEen, CARTER e HAWKInG [BCH73], possuem forte analogia com as 4 leis da termodinâmica:

LEI 0 [BCH73]: A gravidade de superfície $\kappa$ é constante ao longo do horizonte de eventos de um buraco negro estacionário ${ }^{1} \leftrightarrow$ um banho térmico em equilíbrio possui temperatura $T$ constante ao longo de toda a sua extensão.

LEI 1 [BCH73]: $\delta M=\Omega_{H} \delta J+\frac{1}{8 \pi} \kappa \delta A$, onde $M$ é a massa do buraco negro, $\Omega_{H}$ é o valor da componente axial do campo de KILling tipo tempo tangente ao horizonte, $J$ o momento angular do buraco negro e $A$ a área d(e uma seção espacial d)o horizonte de eventos. As variações da métrica implícitas na fórmula são também (quase-)estacionárias ${ }^{1} \leftrightarrow \delta Q=P \delta V+T \delta S$, onde $Q$ é a quantidade de calor, $P$ a pressão, $V$ o volume e $S$ a entropia.

LEI 2 [Haw71]: Teorema de Área - a área d(as seções espaciais d)o horizonte de eventos não pode decrescer ao longo da evolução de CAUCHY de um buraco negro nãoestacionário satisfazendo a condição de energia nula ${ }^{2} \leftrightarrow$ a entropia num sistema fechado não pode decrescer por meio de nenhum processo.

LEI 3 [Isr86]: Um buraco negro não-estacionário suficientemente regular e satisfazendo a condição de energia fraca não pode se tornar extremal, i.e., ficar sem superfícies aprisionadas (i.e., superfícies acausais de codimensão 2 tais que ambas as congruências nulas normais possuem expansão negativa) externas ao horizonte de eventos em tempo (retardado) finito, implicando $\kappa \rightarrow 00^{3} \leftrightarrow$ não é possível atingir $T=0$ em tempo finito por nenhum processo físico.

1 Embora as leis 0, 1 e 2 sejam formuladas em [BCH73], somente as leis 0 e primeira são demonstradas nesta referência, para perturbações de buracos negros estacionários (a terceira lei é apenas sugerida numa forma rudimentar).

${ }^{2} \mathrm{O}$ enunciado e prova da segunda lei foram elaborados anteriormente por HAWkING [Haw71] num contexto não-estacionário.

3 A terceira lei foi formulada da presente maneira precisa e demonstrada por IsRAEL [Isr86]. Lembrar que a formulação de Nernst para a terceira lei, i.e., $S=0 \mathrm{em} T=0$, não é universalmente válida (um exemplo típico é o gelo, que possui uma entropia residual em $T=0$ em virtude das pontes de hidrogênio. Tal fato foi estabelecido de maneira exata em duas dimensões por LiEB [Lie67a, Lie67b]. Note que o gás de Bose livre, que sofre condensação de Bose-Einstein em $T=0$ e, portanto, possui degenerescência no estado fundamental, não constitui outro (contra-)exemplo, pois o comportamento assintótico dessa degenerescência na função de partição à medida que $T \searrow 0$ é apenas polinomial, e não exponencial como no caso do gelo, resultando em $S=0$ se adotarmos a definição de BoLTZMANN para a entropia. Agradeço ao Prof. Walter Wreszinski por chamar minha atenção para este ponto), e tampouco seu análogo "natural" para buracos negros o é [Wal97]. 
Em particular, a área do horizonte de um buraco negro corresponde à entropia. BeKEnStein [Bek74] sugeriu que tal identificação era mais que uma mera analogia a entropia de um buraco negro em equilíbrio seria, de fato, dada pela fórmula

$$
S=\frac{A_{h o r}}{4}
$$

e uma generalização da segunda lei da termodinâmica, levando em conta tanto a entropia da matéria (fora do buraco negro) quanto a entropia do buraco negro, deveria ser válida. Este cenário, sugerido pelo Teorema de Área de HAWking [Haw71], não é necessariamente válido classicamente; entretanto, o trabalho de HAwKING [Haw75] mostrou ${ }^{1}$ que um campo quântico acoplado ao campo gravitacional de um buraco negro termaliza, após um intervalo de tempo suficientemente longo, precisamente na temperatura $T=\frac{\kappa}{2 \pi}$, sugerida pela primeira lei da dinâmica de buracos negros! Este resultado é surpreendente, pois esta última é uma quantidade puramente geométrica, o que indica um caráter universal (i.e., independente do modelo de campos quânticos que acoplamos ao campo gravitacional clássico) para as leis da termodinâmica de buracos negros. ${ }^{2}$ Motivado por tal resultado, 'T HoofT [Hoo93] propôs levar a idéia de BEKEnStein às últimas conseqüências: os graus de liberdade microscópicos de uma teoria quântica de gravitação estão completamente codificados na fronteira do volume do espaço-tempo onde tal teoria é definida. Tal princípio, formalizado posteriormente por Susskind [Sus95], é denominado princípio holográfico.

Na descrição integrada das Teorias de Gauge e da gravitação proposta pela Teoria de Cordas, dois resultados sugerem que esta teoria pode fornecer uma possível realização do princípio holográfico: o cálculo microscópico da entropia de BEKENSTEIN feito por Strominger e VAfa [SV96], e, principalmente, o trabalho de MALDACEnA [Mal98], que, por meio de um (limite efetivo de um) modelo de cordas no espaço-tempo de fundo $A d S_{5} \times S^{5}$, onde o fator $A d S_{5}$ é o espaço-tempo anti-DE SitTer (AdS) em cinco dimensões, obteve o multipleto de uma teoria de gauge supersimétrica e conformalmente invariante com grupo estrutural $S U(N)$ no limite $N \rightarrow \infty$, vivendo na fronteira conforme de $A d S_{5}$. MALDACENA conjeturou que a relação estabelece uma correspondência biunívoca entre as duas teorias, mesmo fora do limite efetivo. Tal correspondência tornou-se conhecida na literatura como correspondência AdS/CFT. A

\footnotetext{
${ }^{1} \mathrm{~A}$ aproximação de óptica geométrica para a função de dois pontos próxima ao horizonte de um buraco negro de SchwarzSchild, adotada por HAwking em [Haw75], é difícil de justificar, pois o comportamento da métrica nessa região implica num índice de refração que varia muito rápido ao longo da coordenada temporal relevante. Um cálculo conceitualmente preciso da radiação de HaWkING foi obtido por FREDENHAGEN e HAAG [FH90].

${ }^{2}$ Do ponto de vista do aparentado efeito UNRUH, isto não é tão surpreendente, tendo em vista os resultados de Bisognano e Wichmann no contexto de campos quânticos de Wightman [BW75, BW76, Sew82]. Entretanto, tal comparação exige cuidado, pois as circunstâncias do efeito HAWKING são diferentes em vários aspectos [Wa194].
} 
formulação desta em termos das funções de $k$ pontos foi obtida por GUBSER, KLEBANOV e POLYAKOV [GKP98] num caso particular, e mais em geral por WitTEN [Wit98]. Nestes dois trabalhos, mostrou-se que as funções (de ScHWinger) de $k$ pontos para a teoria de gauge no limite acima podiam ser obtidas a partir de variações da ação (Euclidiana) clássica de supergravidade ao redor de seu valor na geometria de fundo, mediante variação das condições de contorno no infinito conforme. Mais ainda, a prescrição de WitTen para o funcional gerador da teoria conforme permitiu refrasear a correspondência AdS/CFT unicamente em termos de teorias de campos usuais, sem referência a cordas. Por meio desta formulação, a conjectura de MALDACENA foi testada com sucesso em uma miríade de casos particulares. ${ }^{3}$

A formulação de WiTTEN para a correspondência AdS/CFT abriu caminho para a seguinte pergunta: seria possível estabelecer uma correspondência entre Teorias Quânticas de Campos (TQC) em AdS e teorias quânticas de campos conformalmente covariantes em seu infinito conforme, unicamente a partir dos princípios fundamentais da TQC? A pergunta de fato tem resposta positiva: dentro de uma versão da formulação axiomática de Wightman [SW00] para campos quânticos em AdS, uma correspondência entre funções de $k$ pontos no interior deste último e funções de $k$ pontos conformalmente invariantes na fronteira conforme foi estabelecida rigorosamente por BERTOLA, Bros, Moschella e Schaeffer [BBMS00]. Outrossim, uma correspondência entre álgebras de observáveis locais no interior e na fronteira foi demonstrada por REHREN [Reh00], dentro do formalismo algébrico de HAAG e KASTLER [HK64, Haa96] para a TQC (Fúsica Quântica Local) - esta última correspondência é denominada holografia algébrica ou dualidade de REHREN. Embora a relação entre essas duas formulações e a prescrição de WITTEN tenha sido investigada em vários aspectos [DR02, DR03, Reh05], uma pergunta fundamental permaneceu sem resposta: como efeitos gravitacionais são codificados nessas formulações?

Como ambas as versões se referem unicamente a espaços-tempos AdS (pois dependem criticamente de sua estrutura causal), e não a geometrias mais gerais que possuam o mesmo infinito conforme de AdS (i.e., espaços-tempos assintoticamente AdS (AAdS)), até mesmo a formulação per se dessas contrapartes rigorosas num contexto geométrico mais geral torna-se elusiva. Há, inclusive, indícios [AS02] de que, em geometrias $A A d S$ não-triviais, a dualidade de REHREN se torne fisicamente incompatível com testes co-

\footnotetext{
${ }^{3}$ Infelizmente, a atividade fenomenal que se seguiu aos trabalhos fundamentais acerca da correspondência AdS/CFT, citados acima, tornou praticamente impossível elaborar uma bibliografia sequer pretensamente representativa de suas diferentes vertentes (só o trabalho [Mal98] sozinho possui mais de 4.700 citações, segundo dados de 30/07/2007 no banco de dados online SPIRES-HEP). Assim, conformar-nos-emos a citar unicamente trabalhos de relevância direta ao nosso desenvolvimento, à medida que for necessário, e encaminhamos o leitor interessado à homepage do SPIRES-HEP (http://www.slac.stanford.edu/spires/) para outras direções.
} 
nhecidos da conjectura de MALDACEnA, e, como tal, precise ser reformulada. O que nos leva à segunda pergunta, intimamente ligada à anterior: Quanto do arcabouço subjacente à dualidade de REHREN e de suas propriedades sobrevive em espaços-tempos $A A d S$ "genéricos"?

O corpo da presente Tese propõe uma generalização natural da dualidade de REHREN para espaços-tempos AAdS e investiga como as propriedades da versão original são modificadas por efeitos gravitacionais no interior, visando elucidar as duas questões elaboradas acima.

Uma das lições fundamentais da Física Quântica Local é que a informação física de uma teoria quântica de campos está contida não nos campos / observáveis individuais, mas na inclusão relativa das álgebras locais. Em particular, tal informação é extremamente sensível à estrutura causal do espaço-tempo. Assim, é de se esperar que a distorção da estrutura causal no interior de um espaço-tempo AAdS em virtude de efeitos gravitacionais seja "sentida" na teoria "dual" na fronteira. Veremos, após um estudo qualitativo global dessa deformação dentro de um conjunto de hipóteses razoavelmente geral sobre a geometria do interior, que de fato isso ocorre. Mostraremos efeitos de duas naturezas:

1. A implementação das isometrias assintóticas, longe de ser um fato imediato como no caso $A d S$, é atingida dinamicamente como um processo de retorno ao equilíbrio, o que tem por conseqüência a quebra potencial da simetria conforme. Os estados na teoria quântica na fronteira obtidos por esse processo possuem uma estrutura bastante rica, da qual apenas arranhamos a superfície, e cujas propriedades térmicas que se desviam do equilíbrio codificam detalhes não-triviais da geometria do interior.

2. A estrutura de setores de superseleção é radicalmente modificada por efeitos gravitacionais. Dentro da hipótese de que regiões arbitrariamente pequenas no interior possuem observáveis não-triviais, mais outras hipóteses naturais dentro do contexto da teoria algébrica de setores de superseleção [Ara99, Haa96], mostraremos que a teoria no interior adquire excitações solitônicas localizadas em paredes de domínio, similares às D-branas que ocorrem na formulação original da correspondência AdS/CFT, sinalizando a quebra espontânea de simetrias internas. O aumento não-trivial das álgebras locais na fronteira necessário à incorporação dos "intertwiners" entre os diferentes vácuos parciais faz com que estas álgebras adquiram elementos não-locais (i.e., estendidos) implementando simetrias internas na região onde cada vácuo parcial difere do vácuo total. E, precisamente por causa desses elementos, torna-se fundamentalmente impossível, ao contrário do que ocorre no caso do espaço-tempo AdS, reconstruir completamente a teoria 
quântica no interior a partir unicamente da teoria conforme na fronteira (Subseção 4.4.3, página 97$)$.

Colocamos ênfase neste trabalho em resultados robustos, matematicamente rigorosos e independentes de um modelo específico de campos quânticos, em virtude do caráter universal de vários aspectos da correspondência AdS/CFT. Não investigaremos em detalhe, todavia, a relação do formalismo proposto aqui com a prescrição de WitTEn - tal tarefa encontra-se além do escopo deste trabalho.

\section{Sobre a estrutura desta Tese}

Esta Tese possui três partes. Na Parte I, que compreende os Capítulos 1 e 2, desenvolvemos a parte geométrica do trabalho. Na Parte II, que compreende os Capítulos 3 e 4, apresentamos a nossa proposta de generalização da dualidade de REHREN dentro de um formalismo geral para TQC em espaços-tempos curvos que incorpora covariância e localidade de maneira funtorial, e estudamos consequências universais (i.e., independentes de modelos específicos) dos pontos de vista dinâmico e estrutural. A Parte III consiste apenas da Coda, que conclui o trabalho.

No Capítulo 1, iniciaremos um tratamento sistemático dos espaços-tempos anti-DE SitTer (AdS) e assintoticamente anti-DE SitTer (AAdS). Investigaremos, dentro de um conjunto de hipóteses acerca do comportamento global das geodésicas nulas, como a estrutura causal é globalmente modificada por efeitos gravitacionais consistentes com as condições de contorno no infinito (Proposição 1.8, página 21) - o efeito se deve principalmente ao atraso temporal gravitacional das geodésicas nulas atravessando o interior (Teorema 1.3, página 17). Alguns desses resultados não são novos [Woo94, GW00], mas a técnica do atraso temporal gravitacional é fundamental para a maioria dos desenvolvimentos geométricos a seguir. Definiremos uma generalização simples das regiões tipo cunha ("wedge"), empregadas por REHREN em [Reh00], para espaços-tempos AAdS (Definição 1.5, página 15) - tais regiões generalizam, num certo sentido, a cunha de Rindler $\left\{x^{1}>\left|x^{0}\right|\right\}$ no espaço-tempo de Minkowski, e constituem o protótipo de um horizonte de eventos de um buraco negro assintoticamente estacionário num fundo com constante cosmológica negativa. Mostraremos como é possível reconstruir a topologia do interior por meio de intersecções de cunhas "envelopando" diamantes causais suficientemente pequenos (Subsubseção 1.3.1.3, página 24). Este resultado é original [Rib07], e a demonstração envolve uma interessante aplicação do método empregado por Penrose [HE73, Wal84] para provar a existência de singularidades como resultado do colapso gravitacional, juntamente com um argumento de compacidade, na obtenção de um princípio geométrico de máximo que garante a envoltória precisa (Teorema 1.14, página 28). A apresentação deste Capítulo possui vários aprimoramentos em relação a 
[Rib07].

No Capítulo 2, investigaremos a dinâmica das equações de Einstein em espaçostempos AAdS (Seção 2.2, página 33). Tal discussão visa estimar de maneira mais quantitativa efeitos gravitacionais não-triviais em termos de quantidades geométricas definidas na fronteira conforme. Também construímos de maneira intrínseca famílias de isometrias assintóticas ("boosts") naturalmente associadas a cada cunha AAdS (Proposição 2.5, página 47), e, mais em geral, famílias de difeomorfismos assintoticamente conformes a qualquer diamante relativamente compacto em espaços-tempos causalmente simples (fórmulas (2.42)-(2.44), página 47). A tais famílias, é possível associar uma gravidade de superfície no horizonte de cada cunha ou diamante (Definição 2.2, página 53) e, assim, formular um análogo assintótico da lei zero da (termo)dinâmica de buracos negros (Teorema 2.6, página 53), e resultados correspondentes para a segunda lei (contida na Proposição 1.8, página 21, e devidamente reinterpretada no contexto deste Capítulo) e a caracterização de processos reversíveis (Teorema 2.7, página 56, que segue diretamente da Proposição 1.8 e da Observação 1.7, página 22). A construção apresentada é suficientemente robusta para ser implementada em situações não necessariamente ligadas ao contexto geométrico da correspondência AdS/CFT.

No Capítulo 3, introduziremos uma generalização do formalismo de HAAG e KASTLER para espaços-tempos curvos, proposta por BRUnetTi, FredenhaGen e VerCH [BFV03] e formulada em termos da linguagem de categorias e funtores.

No Capítulo 4, apresentamos de maneira precisa nossa proposta de generalização para a dualidade de ReHren. Para tal, é necessário adaptar as construções do Capítulo 3 ao presente contexto geométrico, o que demanda a imposição de condições de contorno de maneira covariante. Assim, iniciamos nossa discussão com o tratamento algébrico desta questão proposto por SOMmer [Som06], que investiga como é possível estender uma teoria quântica localmente covariante no sentido de BRUNETTi ET AL. para espaços-tempos não globalmente hiperbólicos (Definição 4.1, página 72). Segue-se o exemplo de teorias quânticas locais em espaços-tempos anti-DE SITTER - aqui, procuramos inserir a formulação proposta por BuchHolz, Florig e Summers [BFS00] dentro de um contexto localmente covariante. A vantagem desta formulação, além do número mínimo de premissas, é a imposição de condições de contorno no infinito sobre os estados "elementares" na forma de uma condição de estabilidade termodinâmica (fórmulas (4.6) e (4.7), página 75) - que, no caso de espaços-tempos AAdS, é generalizada na forma de uma condição de "retorno ao equilíbrio" (condição (d), página 86). Empregando as técnicas de Borchers e YngVASOn [BY99] (enunciados nos Teoremas 4.1, 4.2 e 4.3, página $77 f f$.), mostraremos que tal condição pode ser entendida como uma prescrição para o comportamento de escala dos observáveis numa vizinhança do infinito (Proposição 4.4, página 80), que pode ser generalizada para espaços-tempos assintoti- 
camente anti-DE SitTer. Com este arcabouço em mãos, a generalização da dualidade de Rehren (Definição 4.3, página 75) que buscamos surge naturalmente (Definição 4.8, página 84). Investigamos a estrutura dos estados da teoria da fronteira obtida por meio dessa generalização, e terminamos por delinear uma correspondência entre os setores de superseleção da teoria quântica no interior e sua dual na fronteira, ligando setores localizados em diamantes (i.e., no sentido de Doplicher, HAAG e RoBerts [Haa96]) na fronteira a setores solitônicos no interior, localizados ao redor de paredes de domínio de codimensão 2, bastante similares a D-branas.

$\mathrm{Na}$ Coda, apresentamos nossas conclusões e listamos possíveis direções futuras de investigação sugeridas pelo presente trabalho.

Um obstáculo pedagógico à concepção da presente Tese foi o amplo espectro de técnicas matemáticas empregadas - geometria Lorentziana global, álgebras de operadores, categorias e funtores. Assim, coletamos a maioria dos conceitos matemáticos necessários em quatro Apêndices, para não desviar a atenção do leitor das idéias centrais do presente trabalho e tornar o texto matematicamente auto-contido.

O Apêndice A sumariza as noções necessárias de geometria Lorentziana e estrutura causal.

O Apêndice B apresenta conceitos básicos de Álgebras de Operadores (C*-álgebras, álgebras de von Neumann e álgebras de Borchers-Uhlmann), inclusive uma introdução minimal à teoria de TOMITA-TAKESAKI, empregada no Capítulo 4 . Esse Apêndice começa com um tratamento em detalhe de *álgebras, visando unificar a apresentação dos resultados e conceitos de Álgebras de Operadores que dependem unicamente da estrutura algébrica.

O Apêndice $\mathrm{C}$ condensa os conceitos básicos de categorias e funtores empregados nos Capítulos 3 e 4. tulo 1.

O Apêndice D pincela rapidamente os conceitos de homotopia necessários no Capí-

Desejo uma boa leitura a todos! 


\section{Lista de Figuras}

1.1 (a) Domínio fundamental de $A d S_{d}$ (vermelho), dado pelo quociente modulo $\mathbb{Z}$, onde o infinito espacial é representado em azul e o domínio (fundamental) de Poincaré é a parte superior do corte pontilhado; (b) Imagem do mergulho conforme de $A d S_{d}$ em $E S U_{d}$ (vermelho). O infinito $\mathscr{I}$ de $A d S_{d}$ é representado em azul e o domínio de Poincaré $\mathscr{P}$ oi $(p)$ é delimitado pelas curvas pontilhadas; (c) $\mathscr{P}_{o i}(p)$ (vista lateral) delimitado pelas linhas pontilhadas em vermelho, com $\mathscr{I}$ representado em azul; (d) Visualização de $\mathscr{I}$ como $E S U_{d-1}$ (vermelho) com o domínio de Minkowski $\mathscr{M} i n(p)$, correspondente à imagem do mergulho conforme de $\mathbb{R}^{1, d-2}$ em $E S U_{d-1}$, em azul. . . . . . . . . . . . . . . . .

1.2 (a) Correspondência entre cunhas no interior e diamantes na fronteira. (b) Um domínio de Minkowski e o domínio de Poincaré correspondente. . . . . . . .

1.3 Atraso temporal gravitacional. As geodésicas nulas que emanam de $p \in \mathscr{I}$ e permanecem em $\mathscr{I}$ (linhas cheias) são focadas no antípoda $\bar{p}$. Enquanto que uma geodésica nula emanando de $p$ e atravessando o interior também teria extremo futuro em $\bar{p}$ em $A d S_{d}$ (linha pontilhada), num espaço-tempo AAdS satisfazendo a hipótese de focalização global do Teorema 1.3 tal geodésica tem extremo futuro em $q \in I^{+}(p, \mathscr{I})$ (linha alternando pontos e traços) . . . . . . . . . . . . .

1.4 Efeito do atraso temporal gravitacional (cujo efeito sobre geodésicas nulas é exemplificado pelas linhas pontilhadas no corte longitudinal (b) de (a)) sobre cunhas e seus complementos causais em espaços-tempos AAdS. Enquanto que, em $A d S_{d}$, o complemento causal da cunha $\mathscr{W}_{p, \bar{q}}$ é a cunha $\mathscr{W}_{q, \bar{p}}$ (vermelho), os complementos causais de ambas as cunhas (respectivamente verde e azul) não mais o são em espaços-tempos AAdS satisfazendo as condições da Proposição 1.8 e, portanto, do Teorema 1.3, e de uma maneira tal que a região aberta $\mathscr{W}_{p, \bar{q}}^{\prime} \cap \mathscr{W}_{q, \bar{p}}^{\prime} \neq \varnothing$ é não-vazia, o que não pode ocorrer em $A d S_{d} \ldots \ldots$. . . . . . . . . . . . . 



\section{Notações e convenções de uso freqüente}

- $\mathbb{Z}, \mathbb{R}, \mathbb{C}$ - respectivamente, o anel de números inteiros e os corpos de números reais e complexos. $\mathbb{Z}_{+/-}, \mathbb{R}_{+/-}$- respectivamente, os inteiros positivos $(>0) /$ negativos $(<0)$ e os reais positivos / negativos. $\overline{\mathbb{Z}}_{+/-}, \overline{\mathbb{R}}_{+/-}-$respectivamente, os inteiros não negativos $(\geq 0) /$ não positivos $(\leq 0)$ e os reais não negativos / não positivos.

- As partes real e imaginária de $z=x+i y \in \mathbb{C}$ são respectivamente denotadas por $\Re z \doteq x$ e $\Im z \doteq y$, e o complexo conjugado de $z$, por $\bar{z} \doteq x-i y$.

- O conjunto das partes de um conjunto $X$ é denotado por $P(X) \doteq\{U \subset X\}$.

- Dados quaisquer conjuntos $A, S, A^{S} \doteq\{a: S \rightarrow A\}$ denota a potência Cartesiana de $A$ por $S$. Em particular, se $S=\{1, \ldots, n\}$, escrevemos $A^{S} \doteq A^{n}=$ $\left\{\left(a_{1}, \ldots, a_{n}\right): a_{i} \in A, \forall i=1, \ldots, n\right\}$.

- Dado um aberto $\mathscr{O}$ num espaço topológico $X, K \Subset \mathscr{O}$ denota que $K$ possui fecho $\bar{K}$ compacto e $\bar{K} \subset \mathscr{O}$.

- Dada uma forma bilinear simétrica não-degenerada $g$ sobre $\mathbb{R}^{d}$, a assinatura de $g$ é dada pelo par de inteiros $(p, q)$ ou pela sua diferença $p-q$, onde $p+q=d$ e $p$ é o número de autovalores negativos de $g$, denominado índice de $g$. Associamos a $g$ a forma bilinear simétrica não-degenerada $g^{-1} \doteq\left[g_{i j}\right]^{-1}$, de mesmo índice, sobre o dual de $\mathbb{R}^{d}$, e a $d$-forma de volume dada por $\sqrt{|g|} \doteq \sqrt{\left((-1)^{p} \operatorname{det}\left[g_{i j}\right]\right)^{\frac{1}{2}}}$, onde $\left[g_{i j}\right]$ é a matriz associada a $g$ numa base ortonormal qualquer.

- Variedades e subconjuntos destas são denotadas por caracteres caligráficos maiúsculos (e.g. $\mathscr{M}, \mathscr{O}, \mathscr{I}$, etc.). Todas as variedades empregadas no texto são $\mathscr{C}^{\infty}$, $\sigma$-compactas (e, portanto, para-compactas), conexas e orientáveis, quando não indicado o contrário (para as noções de topologia envolvidas, ver [Dug66]).

- Dada uma variedade $\mathscr{M}$, denotamos os espaços tangente e cotangente a $p \in \mathscr{M}$ respectivamente por $T_{p} \mathscr{M}$ e $T_{p}^{*} \mathscr{M}$, e os fibrados tangente e cotangente, por $T \mathscr{M}$ e $T^{*} \mathscr{M}$. 
- Mais em geral, escrevemos um fibrado vetorial qualquer sobre uma $\mathscr{M} d$-dimensional como $\mathscr{E} \stackrel{p}{\longrightarrow} \mathscr{M}$, onde $p$ denota a aplicação (submersão sobrejetora) de projeção, $\mathscr{E}$ o espaço total $(D+d)$-dimensional e $\mathscr{M}$ a base. A fibra típica é em geral denotada por $E_{x} \doteq p^{-1}(x) \cong \mathbb{R}^{D}, \forall x \in \mathscr{M}$.

- Dado um campo vetorial $X$, denotamos a derivada de LiE de funções / seções ao longo de $X$ por $£_{X}$.

- Índices tensoriais são dados por caracteres gregos minúsculos $\mu$, $\nu$, etc. quando escritos em termos de coordenadas locais, e dados por caracteres latinos minúsculos $a, b$, etc. quando apenas indicativos do posto do tensor, sem referência a coordenadas (notação abstrata de Penrose e Rindler). Em ambos os casos, a contração de índices segue a convenção de Einstein.

- Denotamos por $\delta_{b}^{a}$ o delta de KRONECKer (= matriz identidade como transformação linear em $\left.T^{(*)} \mathscr{M}\right)$.

- Dado um tensor de posto $(r, s) T_{\nu_{1} \ldots \nu_{s}}^{\mu_{1} \cdots \mu_{r}}$, denotamos respectivamente as partes simétrica e anti-simétrica de $T$ com relação aos índices contravariantes $\mu_{j}, \ldots, \mu_{k}$, $1 \leq j<k \leq r$ por

$$
T_{\nu_{1} \cdots \nu_{s}}^{\mu_{1} \cdots\left(\mu_{j} \cdots \mu_{k}\right) \cdots \mu_{r}} \doteq \frac{1}{(k-j+1) !} \sum_{\pi \in S_{k-j+1}} T_{\nu_{1} \cdots \nu_{s}}^{\mu_{1} \cdots \mu_{\pi(j)} \cdots \mu_{\pi(k)} \cdots \mu_{r}}
$$

$\mathrm{e}$

$$
T_{\nu_{1} \cdots \nu_{s}}^{\mu_{1} \cdots\left[\mu_{j} \cdots \mu_{k}\right] \cdots \mu_{r}} \doteq \frac{1}{(k-j+1) !} \sum_{\pi \in S_{k-j+1}} \operatorname{sgn}(\pi) T_{\nu_{1} \cdots \nu_{s}}^{\mu_{1} \cdots \mu_{\pi(j)} \cdots \mu_{\pi(k)} \cdots \mu_{r}}
$$

onde $S_{l}$ é o grupo de permutações de $l$ elementos e $\operatorname{sgn}(\pi)=1$ caso $\pi$ envolva um número par de trocas de 2 elementos, e -1 se envolver um número ímpar. Analogamente, denotamos respectivamente as partes simétrica e anti-simétrica de $T$ com respeito aos índices covariantes $\nu_{m}, \ldots, \nu_{n}, 1 \leq m<n \leq s$, por $T_{\nu_{1} \cdots\left(\nu_{m} \cdots \nu_{n}\right) \cdots \nu_{s}}^{\mu_{1} \cdots \mu_{r}}$ e $T_{\nu_{1} \cdots\left[\nu_{m} \cdots \nu_{n}\right] \cdots \nu_{s}}^{\mu_{1} \cdots \mu_{r}}$. Se quisermos excluir o intervalo $\mu_{j}, \ldots, \mu_{k}$ da simetrização ou da anti-simetrização dos índices $\mu_{1}, \ldots, \mu_{l}$, escreveremos respectivamente $\left(\mu_{1} \cdots\left|\mu_{j} \cdots \mu_{k}\right| \cdots \mu_{l}\right)$ ou $\left[\mu_{1} \cdots\left|\mu_{j} \cdots \mu_{k}\right| \cdots \mu_{l}\right]$. As fórmulas correspondentes para (anti-)simetrização de um tensor devem ter o fator combinatório $\frac{1}{l !}$ substituído por $\frac{1}{(l-k+j-1) !}$. Todas essas convenções valem sem alteração para índices abstratos. 
- $\mathbb{R}^{p, q}$ denota a variedade semi-Riemanniana $\left(\mathbb{R}^{p+q}, \eta\right)$ (ver Apêndice $\mathrm{A}$ ), onde a métrica $\eta$ é dada pela forma bilinear simétrica não-degenerada de índice $p$

$$
\eta=\operatorname{diag}(-\cdots-+\cdots+) \doteq\left[\begin{array}{ccc|ccc}
-1 & & 0 & & & \\
& \ddots & & & 0 & \\
0 & & -1 & & & \\
\hline & & & +1 & & 0 \\
& 0 & & & \ddots & \\
& & & 0 & & +1
\end{array}\right]
$$

Em particular, o espaço-tempo de Minkowski $d$-dimensional é dado por $\mathbb{R}^{1, d-1}$ ou seja, adotamos a convenção de assinatura $(-+\cdots+)$ (índice 1) para métricas Lorentzianas.

- Dados $x, y \in \mathbb{R}^{d}$, a norma Euclidiana de $x$ é denotada $|x|$, e o produto escalar correspondente, $\langle x, y\rangle$. A medida de LEBEsGue de um subconjunto Boreliano $A$ de $\mathbb{R}^{d}$ é denotada $|A|$ (para as noções relevantes de teoria da medida, ver [Mun71, Rud87]).

- $S^{d}$ denota a esfera $d$-dimensional $\left\{x \in \mathbb{R}^{d+1}:|x|=1\right\}$, cuja métrica Euclidiana induzida é denotada $d \Omega_{d}^{2}=\left(d \theta^{1}\right)^{2}+\sin ^{2} \theta^{1}\left(\left(d \theta^{2}\right)^{2}+\sin ^{2} \theta^{2}\left(\cdots\left(\left(d \theta^{d-1}\right)^{2}+\right.\right.\right.$ $\left.\left.\left.\sin ^{2} \theta^{d-1}\left(d \theta^{d}\right)^{2}\right) \cdots\right)\right)=\left(d \theta^{1}\right)^{2}+\sin ^{2} \theta^{1} d \Omega_{d-1}^{2}$, onde $\theta^{I}=\left(\theta^{1}, \ldots, \theta^{d}\right)$ são as coordenadas angulares (esféricas) de $S^{d}$, i.e., $0 \leq \theta^{1}, \ldots, \theta^{d-1} \leq \pi$ e $0 \leq \theta^{d}<2 \pi$.

- A norma de um espaço vetorial normado é denotada \|.\|, com indicação adicional quando necessário.

- O produto escalar de um espaço pré-Hilbertiano (real ou complexo) é denotado $\langle.,$.$\rangle . Convencionamos que, no caso complexo, a forma sesquilinear \langle.,$.$\rangle é linear$ na segunda variável e anti-linear na primeira variável.

- *álgebras, em particular C*- e de VON NEumann, são denotadas por caracteres góticos maiúsculos (e.g. $\mathfrak{F}, \mathfrak{A}, \mathfrak{R}$, etc.). Tais álgebras, se unitais, tem seu elemento identidade denotado por $\mathbb{1}$ (ver Apêndice B).

- Funtores também são denotados por caracteres góticos maiúsculos.

- Comportamento assintótico - dizemos que $f(x)=O(g(x))$ à medida que $x \rightarrow x_{0}$ $\left(x_{0}\right.$ pode ser $\left.\pm \infty\right)$ se existe uma vizinhança $\mathscr{U} \ni x_{0}$ e $C>0$ tais que $|f(x)| \leq$ $C|g(x)|, \forall x \in \mathscr{U}$ e $f(x)=o(g(x))$ à medida que $x \rightarrow x_{0}$ se para todo $\epsilon>0$ existe uma vizinhança $\mathscr{U} \ni x_{0}$ tal que $|f(x)|<\epsilon|g(x)|, \forall x \in \mathscr{U} ; f$ e $g$ são ditas equivalentes (notação: $f(x) \sim g(x)$ ) à medida que $x \rightarrow x_{0}$ se existe uma vizinhança $\mathscr{U} \ni x_{0}$ e $C>0$ tais que $C^{-1} g(x) \leq f(x) \leq C g(x), \forall x \in \mathscr{U}$. Dada 
uma seqüência de funções $\left\{g_{k}\right\}_{k \in \mathbb{Z}_{+}}$, dizemos que $f$ é soma assintótica de $g_{k}, k \in$ $\mathbb{Z}_{+}$à medida que $x \rightarrow x_{0}$ (notação: $\left.f \sim_{x \rightarrow x_{0}} \sum_{k=1}^{\infty} g_{k}\right)$ se $\left|f-\sum_{k=1}^{K} g_{k}\right|=O\left(g_{K+1}\right)$, para todo $K$.

- $\mathscr{D}(\mathscr{O})$ denota o espaço das funções $\mathscr{C}^{\infty}$ de suporte compacto em $\mathscr{O}$ (funções teste), e $\mathscr{S}\left(\mathbb{R}^{d}\right)$, das funções $\mathscr{C}^{\infty}$ cujas derivadas de ordem $\geq 0$ tendem a 0 mais rápido que qualquer polinômio em $x$ à medida que $|x| \rightarrow \infty$ (funções teste temperadas ou de SchwARTZ). Escrevemos também, seguindo L. SchwARTZ, $\mathscr{E}(\mathscr{O})=\mathscr{C}^{\infty}(\mathscr{O})$. Empregaremos essas diferentes notações alternadamente ao longo do texto. Respectivamente, as distribuições, distribuições temperadas e distribuições de suporte compacto são dadas pelos duais topológicos $\mathscr{D}^{\prime}(\mathscr{O}), \mathscr{S}^{\prime}\left(\mathbb{R}^{d}\right), \mathscr{E}^{\prime}(\mathscr{O})$.

- Quando $\mathscr{O} \subset \mathscr{M}$, onde $(\mathscr{M}, g)$ é uma variedade semi-Riemanniana $d$-dimensional orientável, usamos o elemento de volume invariante $\sqrt{|g|}$ para identificar espaços de funções teste com as densidades ( $=d$-formas, uma vez que assumimos $\mathscr{M}$ orientável) teste correspondentes. Assim, os duais topológicos são distribuições e não densidades distribucionais (i.e., pelo "smearing" $u(f)$ de uma distribuição $u$ com uma função teste $f$, entende-se a expressão $u(f \sqrt{|g|})$, uma vez que a métrica é sempre clara em contexto).

- Dado um fibrado vetorial $\mathscr{E} \stackrel{p}{\longrightarrow} \mathscr{M}$, o espaço das seções $\mathscr{C}^{\infty}$ de $\mathscr{E}$ é o $\mathscr{C}^{\infty}(\mathscr{M})$ módulo denotado por $\Gamma^{\infty}(\mathscr{M}, \mathscr{E}) \doteq\left\{\phi: \mathscr{M} \stackrel{\mathscr{C}^{\infty}}{\longrightarrow} \mathscr{E}: p \circ \phi=\mathrm{id}_{\mathscr{M}}\right\}$. Denotando a seção zero de $\mathscr{E}$ (i.e., que assume o valor da origem em cada fibra) simplesmente por 0, definimos o suporte supp $\phi$ de uma seção $\phi$ como o complementar do maior aberto em $\mathscr{M}$ onde $\phi=0$. Denotamos, então, o espaço das seções $\mathscr{C}^{\infty}$ de suporte compacto de $\mathscr{E}$ por $\Gamma_{c}^{\infty}(\mathscr{M}, \mathscr{E})$. 


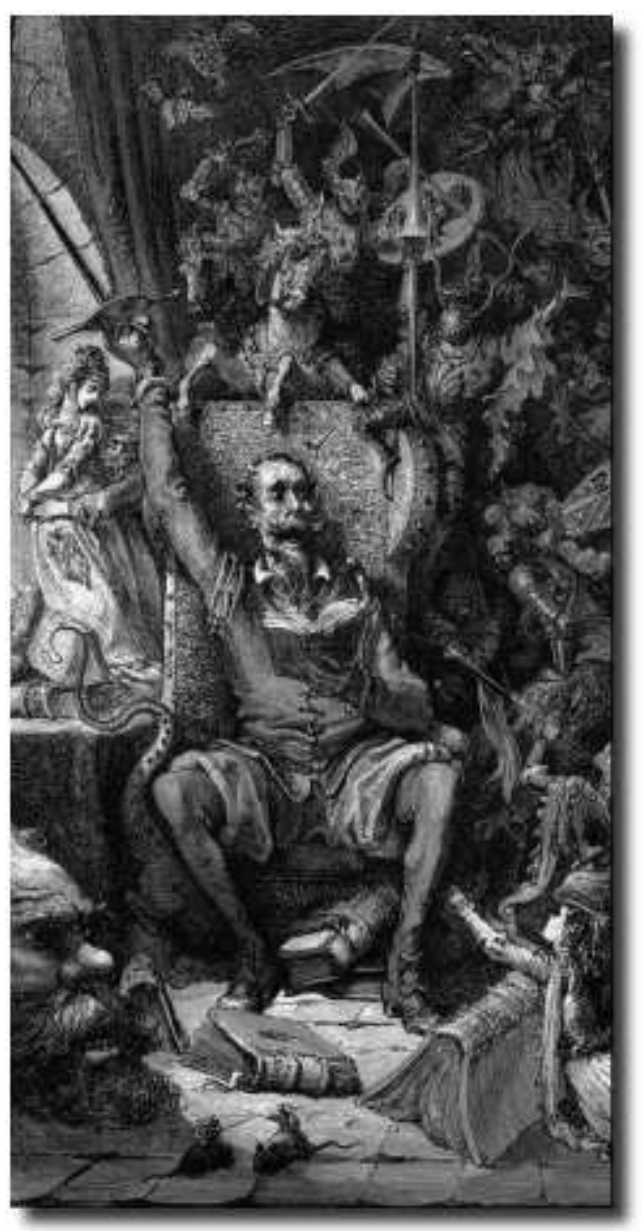

En efeto, rematado ya su juicio, vino a dar en el más estraño pensamiento que jamás dio loco en el mundo, y fue que le pareció convenible y necesario, así para el aumento de su honra como para el servicio de su

república, hacerse caballero andante y irse por todo el mundo con sus armas y caballo a buscar las aventuras y a ejercitarse en todo aquello que él había leído que los caballeros andantes se ejercitaban, deshaciendo todo género de agravio y poniéndose en ocasiones y peligros donde, acabándolos, cobrase eterno nombre y fama. Imaginábase el pobre ya coronado por el valor de su brazo, por lo menos del imperio de Trapisonda; y así, con estos tan agradables pensamientos, llevado del estraño gusto que en ellos sentía, se dio priesa a poner en efeto lo que deseaba. Y lo primero que hizo fue limpiar unas armas que habían sido de sus bisabuelos, que, tomadas de orín y llenas de moho, luengos siglos había que estaban puestas y olvidadas en un rincón. ${ }^{\mathrm{vi}}$

Miguel de Cervantes SaAvedra El ingenioso hidalgo don Quijote de la Mancha ${ }^{\text {vii }}$, Primero Libro, Cap. I

\section{A CORRESPONDÊNCIA ADS/CFT E SUAS MANIFESTAÇÕES GEOMÉTRICAS}





\section{CAPÍtulo 1}

\section{A geometria dos espaços-tempos assintoticamente anti-DE SITTER}

Devia ou não devia contar-lhe, por motivos de talvez. Do que digo, descubro, deduzo. Será, se? Apalpo o evidente? Trebusco. Será este nosso desengonço e mundo o plano intersecção de planos - onde se completam de fazer as almas?

JoÃo Guimarães Rosa "O espelho" (Primeiras Estórias viii)

\subsection{Generalidades sobre formas espaciais}

Dentre as métricas Lorentzianas $d$-dimensionais $g$ que resolvem as equações de EinsTEIN sem matéria e com constante cosmológica $\Lambda$

$$
\operatorname{Ric}(g)-\frac{1}{2} R(g) g+\Lambda g=0
$$

as mais simples são aquelas com curvatura seccional constante. ${ }^{1}$ Mais precisamente, se $g$ é tal que sua curvatura seccional

$$
K(g)(X, Y) \doteq \frac{g(\operatorname{Riem}(g)(X, Y) X, Y)}{g(X, X) g(Y, Y)-g(X, Y)^{2}}=C
$$

\footnotetext{
${ }^{1}$ Para $d<4$, estas são as únicas soluções de (1.1)! Ver Apêndice A, fórmula (A.2), página 108 e a discussão que se segue.
} 
para todo par $X, Y$ de vetores tangentes a um ponto $p$ gerando um plano no qual $g$ é uma forma bilinear não-degenerada ${ }^{2}(C$ pode, em princípio, depender de $p)$, então $g$ satisfaz (1.1) se e somente se $C=\frac{2}{(d-1)(d-2)} \Lambda$. Neste caso, temos:

$$
\operatorname{Riem}(g)_{a b c d}=\frac{2 \Lambda}{(d-1)(d-2)}\left(g_{a c} g_{b d}-g_{a d} g_{b c}\right)
$$

$\left(g_{a c} g_{b d}-g_{a d} g_{b c}\right.$ pode ser vista como a métrica induzida por $g$ no espaço tangente de 2-vetores; $\operatorname{Riem}(g)_{a b c d}$, por sua vez, é uma forma bilinear simétrica neste espaço. A identidade (1.3) segue então de (1.2) por polarização). Conversamente, toda variedade Lorentziana $d$-dimensional de curvatura seccional constante $C$ satisfaz (1.1) com $\Lambda=$ $\frac{(d-1)(d-2)}{2} C$. Nestas circunstâncias, podemos invocar dois resultados (ver [O'N83] para a demonstração de ambos):

TeOrema 1.1 ([O'N83]) Sejam duas variedades Lorentzianas d-dimensionais $(\mathscr{M}, g)$ $e\left(\mathscr{M}^{\prime}, g^{\prime}\right)$ de curvatura seccional constante $K(g)=K\left(g^{\prime}\right)=C$. Então, quaisquer pontos $p \in \mathscr{M}, p^{\prime} \in \mathscr{M}^{\prime}$ possuem vizinhanças isométricas. Se $\mathscr{M}^{\prime}$ é geodesicamente completa, a isometria em questão se estende unicamente a uma isometria local $\phi$ : $\mathscr{M} \rightarrow \mathscr{M}^{\prime}$ (i.e., a aplicação tangente $d \phi(p): T_{p} \mathscr{M} \rightarrow T_{p^{\prime}} \mathscr{M}^{\prime}$ é uma isometria, para todo $p \in \mathscr{M})$.

TeOrema 1.2 ([O'N83]) Sejam duas variedades Lorentzianas d-dimensionais $(\mathscr{M}, g)$ $e\left(\mathscr{M}^{\prime}, g^{\prime}\right)$, geodesicamente completas, conexas e de curvatura seccional constante $K(g)=$ $K\left(g^{\prime}\right)=C$. Então, para quaisquer pontos $p \in \mathscr{M}, p^{\prime} \in \mathscr{M}^{\prime}$ e toda isometria $L: T_{p} \mathscr{M} \rightarrow$ $T_{p^{\prime}} \mathscr{M}^{\prime}$, existe uma única aplicação isométrica de recobrimento (ver Definição D.3, página 159) $\phi: \mathscr{M} \rightarrow \mathscr{M}^{\prime}$ tal que $d \phi(p)=L$.

DEFINIÇÃO 1.1 Uma variedade Lorentziana geodesicamente completa e de curvatura seccional constante é dita ser uma forma espacial.

Segue do Teorema 1.2 que toda forma espacial simplesmente conexa é unicamente determinada por $\Lambda$. Listamos abaixo tais soluções.

$\Lambda>0$ - Espaços-tempos DE SiTTER (notação: $d S_{d}(\Lambda)$ ), dado pelo hiperbolóide espacialmente compacto

$$
d S_{d}(\Lambda) \doteq\left\{X \in \mathbb{R}^{1, d}: \eta(X, X)=R^{2}\right\}, R=\sqrt{\frac{(d-1)(d-2)}{2 \Lambda}} .
$$

\footnotetext{
${ }^{2} \mathrm{O}$ denominador em (1.2) denota o quadrado da área Lorentziana do paralelogramo com lados $X$ e $Y$.
} 
Seu grupo de isometrias é o grupo de DE SitTer $O(1, d)$, com componente conexa à identidade ${ }^{3} S O_{e}(1, d)$.

$\Lambda=0$ - Espaço-tempo de Minkowski $\mathbb{R}^{1, d-1}$. Seu grupo de isometrias é o grupo de POINCARÉ $O(1, d-1) \ltimes \mathbb{R}^{d}$, com componente conexa à identidade $S O_{e}(1, d-1) \ltimes$ $\mathbb{R}^{d} \doteq \mathscr{P}_{+, d}^{\uparrow}$.

$\Lambda<0$ - Espaços-tempos anti-DE SitTer (notação: $A d S_{d}(\Lambda)$ ), dado pelo recobrimento universal (ver Apêndice D, Definição D.3, página 159) do hiperbolóide temporalmente compacto

$$
\begin{gathered}
(1.5) A d S_{d}(\Lambda) \doteq\left\{X \in \mathbb{R}^{2, d-1}:\left(X^{0}\right)^{2}-\left(X^{1}\right)^{2}-\cdots-\left(X^{d-1}\right)^{2}+\left(X^{d}\right)^{2}=R^{2}\right\} \\
R=\sqrt{-\frac{(d-1)(d-2)}{2 \Lambda}}
\end{gathered}
$$

Seu grupo de isometrias é o grupo anti-DE SiTTER, dado no domínio fundamental $(1.5)$ por $O(2, d-1)$, com componente conexa à identidade $S O_{e}(2, d-1)$.

OBSERVAÇÃO 1.2 Em ligeiro abuso de notação, omitiremos em geral a constante cosmológica ao empregarmos a notação acima para as diferentes formas espaciais simplesmente conexas (i.e., apenas $(A) d S_{d}$ ao invés de $(A) d S_{d}(\Lambda)$ ), sempre que tal prática não causar confusão. Contrariamente a uma prática recorrente na literatura, não identificamos $A d S_{d}$ com o domínio fundamental (1.5), pois este último tem papel mínimo ao longo deste trabalho, além de causar complicações técnicas desnecessárias.

Todas as soluções acima são maximalmente simétricas: a álgebra de LiE dos campos de Killing de cada forma espacial possui dimensão maximal $\frac{d(d+1)}{2}$ (conversamente, toda variedade Lorentziana conexa cuja álgebra de LiE de campos de KiLling possui dimensão maximal possui curvatura seccional constante). Por um resultado padrão [O'N83], cada campo de KILLING estende-se unicamente a um grupo uniparamétrico de isometrias, donde segue que o grupo de LIE de isometrias de uma forma espacial possui também dimensão $\frac{d(d+1)}{2}$. Mais ainda: sejam dois pontos $p, q$ de uma forma espacial $\mathscr{M}$. Podemos ligá-los por uma seqüência finita de segmentos geodésicos $\gamma_{i}, i=1, \ldots, k$ com parâmetros afins $\lambda_{i} \in[0,1]$ - neste caso, $\gamma_{i}(1)=\gamma_{i+1}(0), \gamma_{1}(0)=p$ e $\gamma_{k}(1)=q$ - recobrindo qualquer segmento de curva ligando $p$ a $q$ com um número finito de vizinhanças normais. Considere o ponto médio $\bar{p}_{i}$ de $\gamma_{i}$, e a isometria $L_{i}: T_{\bar{p}_{i}} \mathscr{M} \rightarrow T_{\bar{p}_{i}} \mathscr{M}$ dada por $-\mathrm{id}_{\bar{p}_{\bar{p}} \mathscr{M}}$. Pelo Teorema 1.1, cada $L_{i}$ determina uma isometria $\phi_{i}: \mathscr{M} \rightarrow \mathscr{M}$, neste caso satisfazendo $\phi_{i}\left(\gamma_{i}(0)\right)=\gamma_{i}(1)$. Por fim, $\phi=\phi_{k} \circ \cdots \circ \phi_{1}$ é uma isometria ligando $p$ a $q$. Ou seja, quaisquer pontos $p, q$ estão ligados por uma isometria, i.e., toda forma

\footnotetext{
${ }^{3}$ Para variedades Lorentzianas orientáveis e orientáveis no tempo, esta é dada pelas isometrias próprias (que preservam a orientação do elemento de volume, i.e., sua aplicação tangente possui determinante 1) e ortócronas (que preservam a orientação temporal).
} 
espacial é um espaço homogêneo (i.e., a ação do grupo de isometrias é transitiva). Em particular, o grupo de isometrias age em formas espaciais sem pontos fixos, i.e., dado qualquer ponto $p$, existe uma isometria $\phi$ tal que $\phi(p) \neq p$.

\subsection{Geometria de $A d S_{d}$ : condições de contorno no infinito}

Podemos escrever um sistema global de coordenadas esfericamente simétricas no espaço para $A d S_{d}$. Considere $(\tau, r, \mathbf{e}) \in \mathbb{R} \times \overline{\mathbb{R}}_{+} \times S^{d-2}$. Escrevendo

$$
\left\{\begin{array}{l}
X^{0}=\sqrt{R^{2}+r^{2}} \sin t \\
\mathbf{X}=r \mathbf{e} \\
X^{d}=\sqrt{R^{2}+r^{2}} \cos t
\end{array}, R\right. \text { dado por (1.5), }
$$

a métrica de $A d S_{d}$ fica

$$
d s^{2}=-R^{2}\left(1+\frac{r^{2}}{R^{2}}\right) d t^{2}+\left(1+\frac{r^{2}}{R^{2}}\right)^{-1} d r^{2}+r^{2} d \Omega_{d-2}^{2} .
$$

O domínio fundamental é obtido com a restrição $-\pi<t \leq \pi$. Como o recobrimento é, assim, obtido "desenrolando-se" a coordenada temporal $\tau$, denominaremos a carta global (1.6-1.7) carta de recobrimento. Um sistema de coordenadas definido apenas no domínio $\left\{X: X^{d-1}+X^{d}>0\right\}$ (denominado domínio (fundamental) de PoINCARÉ), mas bem mais conveniente para a análise de vários aspectos geométricos de $A d S_{d}$, é dado por $\left(x^{\mu}, z\right) \in \mathbb{R}^{1, d-2} \times \mathbb{R}_{+}$, se escrevermos

$$
\left\{\begin{array}{l}
X^{\mu}=\frac{R}{z} x^{\mu}(\mu=0, \ldots, d-2) \\
X^{d-1}=R\left(\frac{1-z^{2}}{2 z}+\frac{1}{2 z} x_{\mu} x^{\mu}\right) \\
X^{d}=R\left(\frac{1+z^{2}}{2 z}-\frac{1}{2 z} x_{\mu} x^{\mu}\right)
\end{array}\right.
$$

Neste caso, (1.7) fica com a seguinte forma:

$$
d s^{2}=\frac{R^{2}}{z^{2}}\left(\eta_{\mu \nu} d x^{\mu} d x^{\nu}+d z^{2}\right) .
$$

Esta carta é denominada carta horocíclica ou de Poincaré. Pode-se ver pela fórmula (1.9) que cada hipersuperfície de tipo tempo no domínio de POINCARÉ dada por $z=$ const. é conforme a $\mathbb{R}^{1, d-2}$ por um fator 


$$
\left(X^{d-1}+X^{d}\right)^{2}=\frac{R^{2}}{z^{2}}
$$

Assim, o domínio de PoINCARÉ corresponde à metade $z>0$ de $\mathbb{R}^{1, d-1} \ni\left(x^{\mu}, x^{d}-1 \doteq\right.$ $z)$, a menos do fator conforme (1.10). Podemos obter a ação de vários subgrupos de $S O_{e}(2, d-1)$ sobre $A d S_{d}$ a partir do subgrupo d(a componente conexa d)o grupo conforme $S O_{e}(2, d)$ de $\mathbb{R}^{1, d-1}$ que preserva individualmente cada metade $\left\{\left(x^{\mu}, x^{d-1}\right)\right.$ : $\left.x^{d-1} \gtrless 0\right\}$ usando essa parametrização, a saber:

- Subgrupo de POINCARÉ:

$$
\left(z, x^{\mu}\right) \mapsto\left(z, \Lambda_{\nu}^{\mu} x^{\nu}+a^{\mu}\right), \Lambda \in S O_{e}(1, d-2), a \in \mathbb{R}_{d}
$$

Este subgrupo preserva as hipersuperfícies de $z$ constante, e age em cada uma como o grupo de Poincaré age em $\mathbb{R}^{1, d-2}$.

- Subgrupo de dilatações:

$$
\left(z, x^{\mu}\right) \mapsto\left(\lambda z, \lambda x^{\mu}\right), \lambda \in \mathbb{R}_{+}
$$

As isometrias restantes, que não preservam o domínio fundamental de POINCARÉ, serão apresentadas na Subseção 1.2.1.

\subsubsection{Estrutura do infinito conforme}

Consideremos a parametrização de recobrimento (1.6). Mostraremos agora que $A d S_{d}$ possui um infinito conforme no sentido da Definição A.3. Efetuando a mudança de variáveis

$$
\begin{gathered}
\tau=t, u=2\left(\sqrt{1+\frac{r^{2}}{R^{2}}}\right)-\frac{r}{R}, d r=-R\left(\frac{1}{u^{2}}+\frac{1}{4}\right) d u \\
r \in[0,+\infty) \leftrightarrow u \in(0,2],
\end{gathered}
$$

a métrica (1.7) fica

$$
d s^{2}=\frac{R^{2}}{u^{2}}\left[-\left(1+\frac{u^{2}}{4}\right)^{2} d t^{2}+d u^{2}+\left(1-\frac{u^{2}}{4}\right)^{2} d \Omega_{d-2}^{2}\right] .
$$

O infinito espacial de $A d S_{d}$ é obtido tomando-se $r \rightarrow \infty$, que corresponde ao limite $u \searrow 0$. A métrica do completamento conforme $d \bar{s}^{2}=u^{2} d s^{2}$, neste limite, fica

$$
d \bar{s}^{2}=R^{2}\left(-d t^{2}+d \Omega_{d-2}^{2}+d u^{2}\right)=R^{2}\left(d u^{2}+d s_{0}^{2}\right),
$$


onde $d s_{0}^{2}$ é a métrica do universo estático de EINSTEIN $(d-1)$-dimensional $\left(E S U_{d-1}\right)$, que é isométrico a $\mathbb{R} \times S^{d-2}$. Para vermos qual a fronteira conforme do domínio fundamental, retornemos às coordenadas $\tau, r$; nota-se que a fronteira é, então, conforme a $\mathbb{R}^{1, d-2}$ por um fator $\left(X^{d-1}+X^{d}\right)^{-2}$. Tomando-se coordenadas projetivas (de DiRACWEYL), temos:

$$
x^{\mu}=\frac{X^{\mu}}{X^{d-1}+X^{d}} \stackrel{r \rightarrow \infty}{\longrightarrow}\left\{\begin{array}{l}
x^{0}=\frac{\sin \tau}{\cos \tau+\mathrm{e}^{d}} \\
\mathbf{x}=\frac{\mathbf{e}}{\cos \tau+\mathrm{e}^{d}}
\end{array} .\right.
$$

Mais precisamente, a fronteira conforme do domínio fundamental de $A d S_{d}$ corresponde à compactificação conforme de $\mathbb{R}^{1, d-2}$ (notação: $c \mathbb{R}^{1, d-2}$ ).

$E S U_{d-1}$, ao contrário de $c \mathbb{R}^{1, d-2}$, não sofre de conflitos de causalidade devido à ação do grupo conforme de $\mathbb{R}^{1, d-2}$, a qual se estende ao recobrimento universal ${ }^{4} \widetilde{S O}_{e}(2, d-1)$. Seu ordenamento causal global é dado por [LM75]

$$
(\tau, \mathrm{e}) \ll \mathscr{I}\left(\tau^{\prime}, \mathrm{e}^{\prime}\right) \text { se e somente se } \tau-\tau^{\prime}>2 \operatorname{Arccos}\left(\mathrm{e} . \mathrm{e}^{\prime}\right),
$$

onde $\operatorname{Arccos}(a)$ corresponde ao ramo principal de $\cos ^{-1}(a)(-\pi<a \leq \pi)$. Em termos da parametrização de POINCARÉ (1.8), cada hipersuperfície de $z$ constante corresponde, em coordenadas projetivas, exatamente a $\mathbb{R}^{1, d-2}$, resultado que se estende a $z=0$. Assim, conversamente a (1.16), podemos reobter a carta global de $E S U_{d-1}$ escrevendo explicitamente o mergulho conforme de $\mathbb{R}^{1, d-2}$ neste último. A saber, reescrevendo a métrica $\eta$ em coordenadas "espacialmente" esféricas

$$
\eta=-\left(d x^{0}\right)^{2}+d \mathbf{x} \cdot d \mathbf{x}=-\left(d x^{0}\right)^{2}+d r^{2}+r^{2} d \Omega_{d-3}^{2}
$$

e passando para coordenadas radiais avançada $(v)$ e retardada $(u)$ no cone de luz

$$
\left.\begin{array}{l}
v \doteq t+r \\
u \doteq t-r
\end{array}\right\} \Rightarrow \eta=-d u d v+\frac{1}{4}(v-u)^{2} d \Omega_{d-3}^{2}
$$

vemos que, mediante a escolha de fator conforme $\Omega^{2}=\frac{4}{\left(1+u^{2}\right)\left(1+v^{2}\right)}$ e as mudanças de coordenadas $(u, v) \mapsto(U, V)$ (dada por $U=2 \arctan u$ e $V=2 \arctan v)$ e $(U, V) \mapsto$ $\left(T \doteq \frac{V+U}{2}, R \doteq \frac{V-U}{2}\right)$, segue que

$$
d s_{0}^{2}=\Omega^{2} \eta=-d T^{2}+d R^{2}+\sin ^{2} R d \Omega_{d-3}^{2}=-d T^{2}+d \Omega_{d-2}^{2},
$$

de acordo com (1.15). Ou seja, a mudança de coordenadas $\left(x^{\mu}\right) \mapsto\left(T, \theta^{1} \doteq R, \theta^{2}, \ldots, \theta^{d-2}\right)$ corresponde do ponto de vista passivo ao que, do ponto de vista ativo, é o mergulho conforme de $\mathbb{R}^{1, d-2}$ em $E S U_{d-1}$ ( $U$ e $V$ são as coordenadas radiais de cone de luz em

\footnotetext{
${ }^{4}$ Modulo o grupo $\mathbb{Z}_{2}$ de reflexões ao redor do infinito espacial de $c \mathbb{R}^{1, d-2}$.
} 
$\left.E S U_{d-1}\right)$. Convém também notar, à luz destas considerações, que $A d S_{d}$, expresso em termos das coordenadas projetivas (1.16) devidamente estendidas ao recobrimento, corresponde a metade de $E S U_{d}$. O domínio de POINCARÉ, por sua vez, corresponde à imagem do mergulho conforme da metade $x^{d-1}>0$ de $\mathbb{R}^{1, d-1}$ em $E S U_{d}$, e seu infinito $\left(x^{d-1}=0\right)$, à imagem do mergulho conforme de $\mathbb{R}^{1, d-2}$ em $E S U_{d-1}$. A análise acima é sintetizada pictorialmente na Figura 1.1.

A partir do que foi dito acima, é imediato identificar o subgrupo restante de $\widetilde{S O}_{e}(2, d-1)$. A conjugação do subgrupo de translações em $x^{\mu}$ (ver (1.11), página 7) pela aplicação de inversão relativística em $\mathbb{R}^{1, d-1}$

$$
I:\left(x^{\mu}, x^{d-1}\right)=\left(x^{\mu}, z\right) \mapsto-\frac{1}{z^{2}+x_{\nu} x^{\nu}}\left(x^{\mu}, z\right), x_{\nu} x^{\nu}=\eta_{\rho \sigma} x^{\rho} x^{\sigma}
$$

resulta nas transformações conformes especiais

$$
\begin{aligned}
\left(x^{\mu}, z\right) & \mapsto I \circ\left(.+b^{\mu}\right) \circ I\left(x^{\mu}, z\right)=\frac{1}{1-2 b_{\mu} x^{\mu}+b^{2}\left(x^{2}+z^{2}\right)} . \\
& .\left(x^{\mu}-b^{\mu}\left(x^{2}+z^{2}\right), z\right), b \in \mathbb{R}^{1, d-2} \ni a^{\mu}, a^{2}:=a_{\mu} a^{\mu} .
\end{aligned}
$$

Tais transformações, se conjugadas por elementos adequados dos subgrupos (1.111.12) e estendidas ao espaço de parâmetros de $\widetilde{S O}_{e}(2, d-1)$, não mais preservam o domínio de Poincaré, mas preservam o infinito de $A d S_{d}$ como um todo. Para $z=0$, (1.18) se reduz às transformações conformes especiais em $\mathbb{R}^{1, d-2}$. Os subgrupos dados por (1.11) e (1.12) sobre as coordenadas projetivas $x^{\mu}$ correspondem, respectivamente, às ações dos grupos de Poincaré e de dilatações em $\mathbb{R}^{1, d-2}$. Em suma, o grupo de isometrias do domínio fundamental de $A d S_{d}$ (resp. $A d S_{d}$ ) corresponde precisamente ao grupo conforme de $(c) \mathbb{R}^{1, d-2}$ (resp. $\left.E S U_{d-1}\right)$.

\subsubsection{Cunhas em AdS e diamantes na fronteira}

Consideremos, em $A d S_{d}$, a seguinte região causalmente completa, denominada $c u$ nha (padrão) (notação: $\left.\mathscr{W}_{0}\right)$ :

$$
\mathscr{W}_{0}=\left\{X \in \mathbb{R}^{2, d-1}: \eta(X, X)=-R^{2} \text { e } X^{d-1}>\left|X^{0}\right|\right\}
$$

ou, em coordenadas de Poincaré $\left(z, x^{\mu}\right)$ :

$$
\mathscr{W}_{0}=\left\{\left(z, x^{\mu}\right): \sqrt{z^{2}+\mathbf{x} \cdot \mathbf{x}}<1-\left|x^{0}\right|, z<1\right\} .
$$


(b)
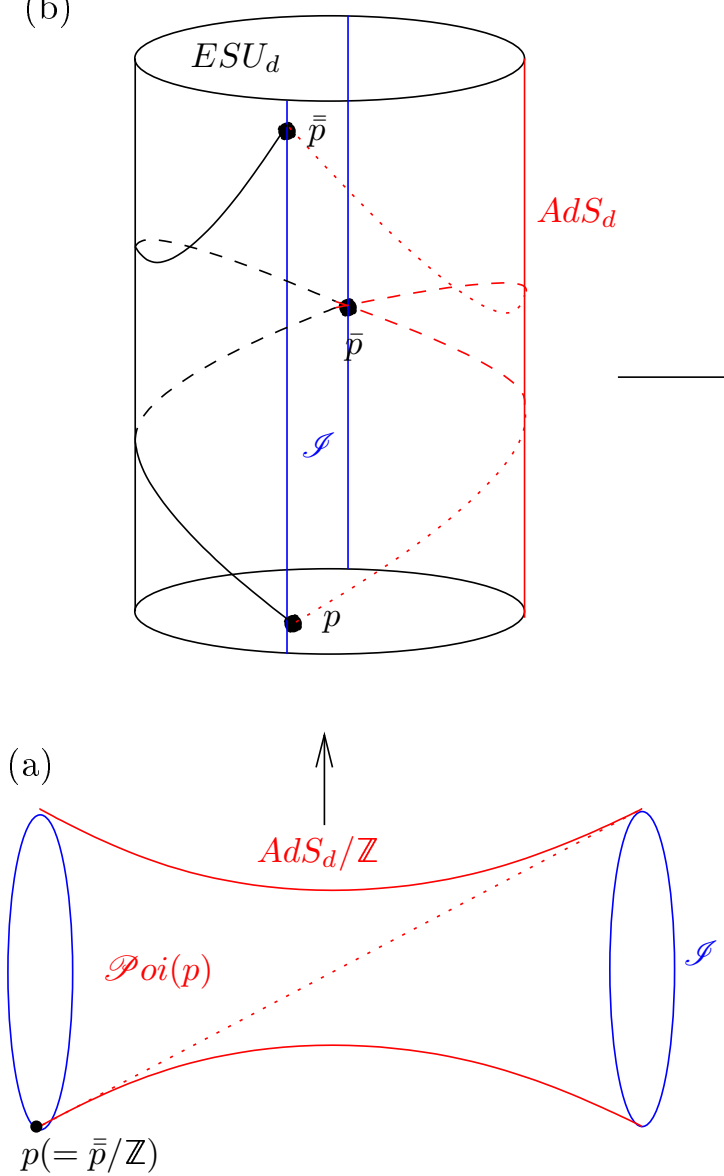

$A d S_{d}$ (c)
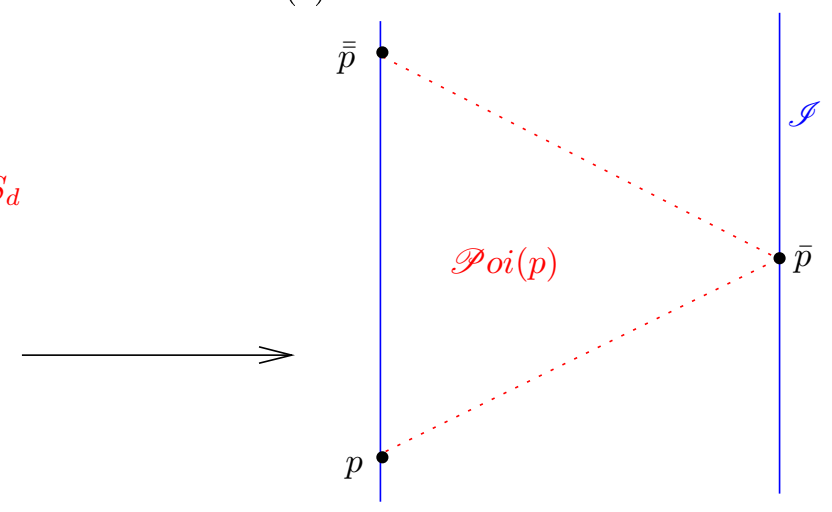

V

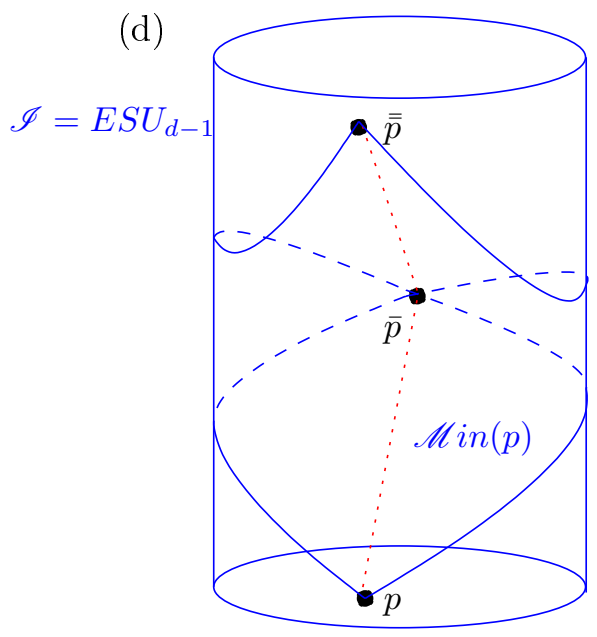

Figura 1.1: (a) Domínio fundamental de $A d S_{d}$ (vermelho), dado pelo quociente modulo $\mathbb{Z}$, onde o infinito espacial é representado em azul e o domínio (fundamental) de Poincaré é a parte superior do corte pontilhado; (b) Imagem do mergulho conforme de $A d S_{d}$ em $E S U_{d}$ (vermelho). O infinito $\mathscr{I}$ de $A d S_{d}$ é representado em azul e o domínio de Poincaré $\mathscr{P}$ oi $(p)$ é delimitado pelas curvas pontilhadas; (c) $\mathscr{P}$ oi $(p)$ (vista lateral) delimitado pelas linhas pontilhadas em vermelho, com $\mathscr{I}$ representado em azul; (d) Visualização de $\mathscr{I}$ como $E S U_{d-1}$ (vermelho) com o domínio de Minkowski $\mathscr{M} i n(p)$, correspondente à imagem do mergulho conforme de $\mathbb{R}^{1, d-2}$ em $E S U_{d-1}$, em azul.

Ao tomarmos o limite $z \rightarrow 0$, nota-se que a intersecção de $\mathscr{W}_{0}$ com a fronteira conforme (notação: $\mathscr{K}_{0}$ ) é dada por

$$
\mathscr{K}_{0}=\left\{x^{\mu}:|\mathbf{x}|<1-\left|x^{0}\right|\right\},
$$

ou seja, $\mathscr{K}_{0}$ corresponde a um diamante (de raio espacial 1 ) em $\mathbb{R}^{1, d-2}$. 
Uma cunha em $A d S_{d}$ é o completamento causal da órbita de um observador uniformemente acelerado. Pela fórmula 1.19, vemos que tais órbitas são dadas pela restrição ao domínio fundamental de $A d S_{d}$ dos "boosts" no plano $X^{0}-X^{d-1}$ :

$$
\begin{aligned}
X^{0} & \mapsto X^{0} \cosh \lambda+X^{d-1} \sinh \lambda ; \\
X^{d-1} & \mapsto X^{0} \sinh \lambda+X^{d-1} \cosh \lambda ; \\
\mathbf{X} & \mapsto \mathbf{X} ; X^{d} \mapsto X^{d}, \lambda \in \mathbb{R} .
\end{aligned}
$$

Em coordenadas de POINCARÉ, é mais fácil visualizar a transformação no mergulho conforme de $A d S_{d}$ em $E S U_{d}$. $\mathscr{W}_{0}$ é a metade $x^{d-1}>0$ de um diamante contido no mergulho conforme de $\mathbb{R}^{1, d-1}$ em $E S U_{d}$, com vértices em $\mathscr{I}$. (1.22) corresponde ao único subgrupo uniparamétrico de $\widetilde{S O}_{e}(2, d-1)$ que preserva esse diamante. A ação deste é simétrica por rotações ao redor do eixo determinado pelos vértices, e, restrita ao plano $x^{0}-z$, fica

$$
z_{ \pm} \mapsto z_{ \pm}(\lambda) \doteq \frac{\left(1+z_{ \pm}\right)-e^{-\lambda}\left(1-z_{ \pm}\right)}{\left(1+z_{ \pm}\right)+e^{-\lambda}\left(1-z_{ \pm}\right)}, \lambda \in \mathbb{R}
$$

onde empregamos as coordenadas no cone de luz $z_{ \pm} \doteq x^{0} \pm z$. O subgrupo de isotropia correspondente para $\mathscr{D}_{0}$ é obtido, digamos, no plano $x_{0}-x_{1}$ substituíndo $z$ por $x^{1}$ e as coordenadas no cone de luz $z_{ \pm}$por $x_{ \pm} \doteq x^{0} \pm x^{1}$ em (1.23). O subgrupo uniparamétrico de $S O_{e}(2, d-1)$ dado por (1.23) corresponde à conjugação $(x, z) \mapsto\left(K^{-1} \circ\left(e^{\lambda}\right.\right.$. $\left.) \circ K\right)(x, z)$ da dilatação $(x, z) \mapsto\left(e^{\lambda} x, e^{\lambda} z\right)$ com a transformação conforme $K$ em $\mathbb{R}^{1, d-1}$ dada pela composição

$$
\mathscr{W}_{0} \ni(x, z) \doteq\left(x^{0}, \mathbf{x}, z\right) \mapsto K(x, z) \doteq I\left(x^{0}-1, \mathbf{x}, z\right)-\left(\frac{1}{2}, \mathbf{0}, 0\right),
$$

onde $I$ é a aplicação de inversão relativística. Nesta forma, fica evidente a simetria rotacional da fórmula (1.23) ao redor do eixo $x^{0}$, invocada acima.

Finalmente, como um prelúdio para a Seção 1.3, passaremos a proceder de maneira independente de coordenadas. Denotando os vértices de $\mathscr{D}_{0}$ por $p_{0}=(-1,0)$ e $q_{0}=(1, \mathbf{0})$, vemos que, se $\overline{A d S_{d}}$ é o fecho conforme de $A d S_{d}$, então $\left(I^{-}\left(p_{0}, \overline{A d S_{d}}\right) \cap\right.$ $\left.I^{+}\left(q_{0}, \overline{A d S_{d}}\right)\right) \cap A d S_{d}=\mathscr{W}_{0}$ e $.\left(I^{-}\left(p_{0}, \overline{A d S_{d}}\right) \cap I^{+}\left(q_{0}, \overline{A d S_{d}}\right)\right) \cap \mathscr{I}=\left(I^{-}\left(p_{0}, \mathscr{I}\right) \cap\right.$ $\left.I^{+}\left(q_{0}, \mathscr{I}\right)\right)=\mathscr{D}_{0}$. Mais em geral, definamos para $p, q \in \mathbb{R}^{1, d-2}, p \ll \mathscr{I} q$ a cunha

$$
\mathscr{W}_{p, q} \doteq\left(I^{-}\left(p, \overline{A d S_{d}}\right) \cap I^{+}\left(q, \overline{A d S_{d}}\right)\right) \cap A d S_{d}
$$

e o diamante

$$
\mathscr{D}_{p, q} \doteq\left(I^{-}\left(p, \overline{A d S_{d}}\right) \cap I^{+}\left(q, \overline{A d S_{d}}\right)\right) \cap \mathscr{I}=I^{-}(p, \mathscr{I}) \cap I^{+}(q, \mathscr{I})
$$


associados a $p, q$. Em particular, $\mathscr{W}_{0}=\mathscr{W}_{p_{0}, q_{0}}$ e $\mathscr{D}_{0}=\mathscr{D}_{p_{0}, q_{0}}$. Denotemos por $u_{p, q}^{\lambda}$ o subgrupo uniparamétrico de isometrias de $A d S_{d}$ que preservam $\mathscr{W}_{p, q}$ e $\mathscr{D}_{p, q}$, dado por (1.23) no caso $p=p_{0}, q=q_{0}$. Este subgrupo é dado por

$$
\begin{aligned}
u_{p, q}^{\lambda}(x, z) & =K_{p, q}^{-1}\left(e^{\lambda} K_{p, q}(x, z)\right), \text { onde } \\
K_{p, q}(x, z) & \doteq K\left(\Lambda_{p, q}\left(x-\frac{x(p)+x(q)}{2}\right), z\right),
\end{aligned}
$$

onde $\Lambda_{p, q}$ é o "boost" de LoRENTz ao redor da origem que torna a direção $\overrightarrow{x(p) x(q)}$ paralela ao eixo $x^{0}$, e $K$ é a aplicação usada para definir o subgrupo de isotropia (1.23) de $\mathscr{W}_{0}$ acima. Estes subgrupos uniparamétricos serão de importância crucial nos Capítulos 2 e 4.

Vamos agora tornar a descrição da imagem do mergulho conforme de $\mathbb{R}^{1, d-2}$ em $E S U_{d-1}$ independente do ponto escolhido para representar o infinito espacial do primeiro. Seja $p \in \mathscr{M} i n(r)$. Todas as geodésicas nulas emanando de $p$ irão se focalizar em um único ponto de $\mathscr{I}$, que constitui o extremo futuro de todas as geodésicas acronais pertencentes a $\partial I^{+}(p, \mathscr{I})$. Esse ponto é denominado antípoda de $p$, denotado por $\bar{p}$. O antípoda de $p$ possui as seguintes propriedades:

$$
\begin{aligned}
\partial I^{+}(p, \mathscr{I}) \backslash\{p\} & =\partial I^{-}(\bar{p}, \mathscr{I}) \backslash\{\bar{p}\} ; \\
\partial I^{+}\left(p, \overline{A d S_{d}}\right) \backslash\{p\} & =\partial I^{-}\left(\bar{p}, \overline{A d S_{d}}\right) \backslash\{\bar{p}\} .
\end{aligned}
$$

Definamos $\mathscr{M} i n(p) \doteq \mathscr{D}_{p, \overline{\bar{p}}}$, o dominio de Minkowski ao futuro de $p \in \mathscr{I}$. Essa região corresponde ao mergulho conforme de $\mathbb{R}^{1, d-2}$ em $\mathscr{I}$ tal que $p$ corresponde ao "infinito" do passado temporal de $\mathbb{R}^{1, d-2}$. $\mathscr{P}_{o i}(p) \doteq \mathscr{W}_{p, \overline{\bar{p}}}$ corresponde ao domínio de uma carta de Poincaré em $A d S_{d}$, sendo portanto denominado domínio de Poincaré ao futuro de $p$. Estendemos as definições (1.24) de cunha e (1.25) de diamante para todos os pares de pontos $p \ll \mathscr{I} q$ tais que $p, q \in \overline{\mathscr{M} i n(r)}$ para algum $r \in \mathscr{I}$, com a mesma notação.

OBSERVAÇÃO 1.3 Uma maneira equivalente de caracterizar cunhas $\mathscr{W}_{p, q}$ e diamantes $\mathscr{D}_{p, q}$ tais que $p, q \in \mathscr{M}$ in $(r)$ para algum $r \in \mathscr{I}$ é a seguinte: como ESU $U_{d-1}$ é globalmente hiperbólico, segue que $\mathscr{D}_{p, q}$ é relativamente compacto e, portanto, globalmente hiperbólico. É possível, então, distinguir três situações possíveis:

1. Não existe $r \in \mathscr{I}$ tal que $p, q \in \overline{\mathscr{M} i n(r)}$ : neste caso, tomemos $r=p$; segue que $\bar{r}=\bar{p} \ll \mathscr{I} q$ e, portanto, $\mathscr{D}_{p, q}$ contém uma superfície de CAUCHY para $\mathscr{I}$. Em particular, as superfícies de CAUCHY de $\mathscr{D}_{p, q}$ são compactas e, mais importante, não-contráteis (para a definição de contratibilidade, ver a Seção D.1, página 157). 
(a)

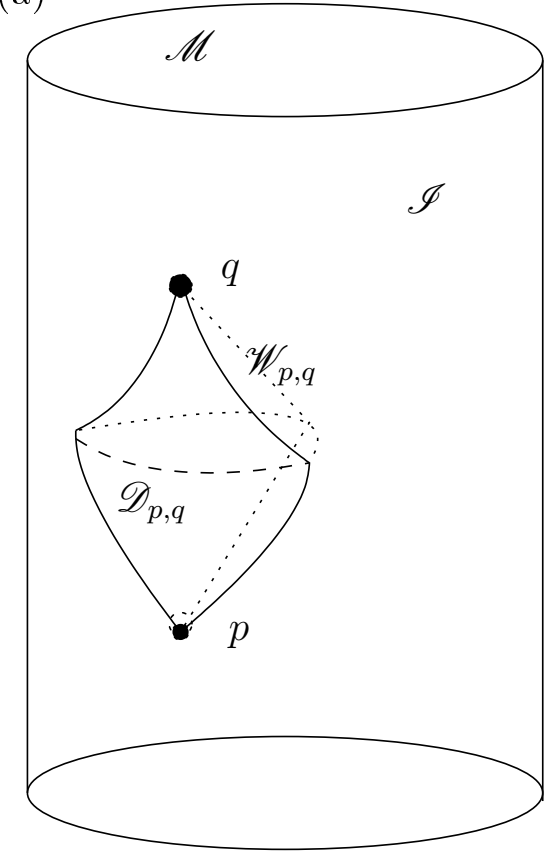

(b)

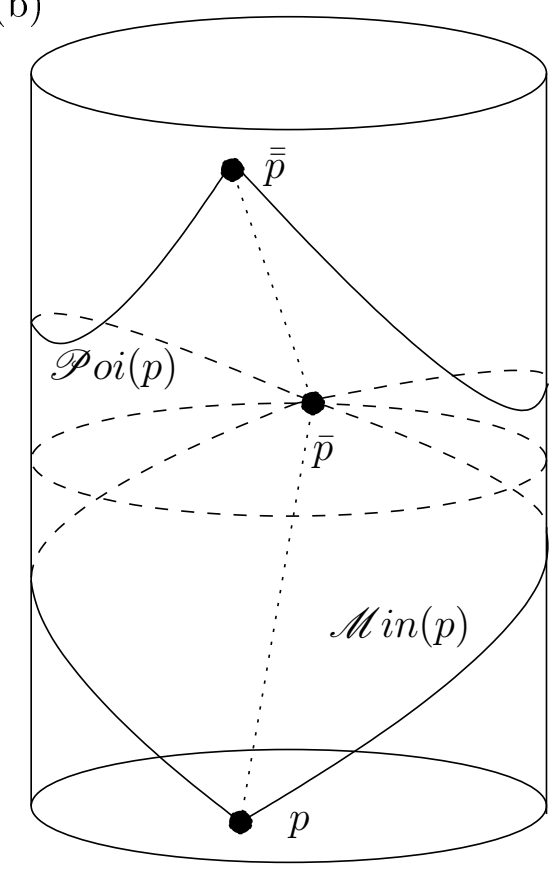

Figura 1.2: (a) Correspondência entre cunhas no interior e diamantes na fronteira. (b) Um domínio de Minkowski e o domínio de Poincaré correspondente.

2. $p, q \in \mathscr{M}$ in $(r)$ para algum $r \in \mathscr{I}:$ como aqui $\mathscr{D}_{p, q}$ pode ser entendido como um diamante em $\mathscr{M} i n(r)$, as superficies de CAUCHY de $\mathscr{D}_{p, q}$ são não-compactas $e$ contráteis.

3. $p, q \in \partial \mathscr{M}$ in $(r)$ para algum $r \in \mathscr{I}:$ este caso limitrofe inclui os próprios domínios de Minkowski, e, por definição, $\mathscr{D}_{p, q}$ pode então ser obtido como o limite $\mathscr{D}_{p, q}=$ $\lim _{n \rightarrow \infty} \mathscr{D}_{p, q_{n}}=\bigcup_{n=1}^{\infty} \mathscr{D}_{p, q_{n}}$, onde novamente escolhemos $r=p e\left\{q_{n}\right\}_{n \in \mathbb{Z}_{+}} \subset$ $\mathscr{M}$ in $(r)$ é uma seqüência de pontos tais que $q_{n} \stackrel{n \rightarrow \infty}{\longrightarrow} q$ e $q_{n} \ll \mathscr{I} q_{n+1} \ll \mathscr{I} q$ para todo $n$. Em particular, $\mathscr{D}_{p, q_{n}} \in \mathscr{M} i n\left(r_{n}\right)$, onde $r_{n} \ll \mathscr{I} r$ é suficientemente próximo de $r=p$. As superfícies de $\mathrm{CAUCHY}$ de $\mathscr{D}_{p, q}$ também são não-compactas e contráteis.

Ressaltamos que o caso 1 não pode de maneira alguma ser englobado por algum limite de uma seqüência crescente de diamantes dentro do caso 2, como no caso 3. Em suma, a coleção de diamantes que nos interessa é aquela composta de diamantes com superfície de CAUCHY contrátil. Os diamantes dentro do caso 2 são, de certa forma, "densos" nessa coleção - dado qualquer $\mathscr{D}_{p, q}$ com superfície de CAUCHY contrátil e qualquer $q^{\prime} \ll_{\mathscr{I}} q$, não importa quão próximo de $q$, temos que $\mathscr{D}_{p, q^{\prime}}$ é um diamante dentro do caso 2. 
A estrutura de $E S U_{d-1}$ implica ainda nos seguintes fatos de grande importância para nós:

1. De (1.27) e (1.28), segue que

$$
\mathscr{W}_{q, \bar{p}}=\left(\mathscr{W}_{p, \bar{q}}\right)_{A d S_{d}}^{\prime} \text { e } \mathscr{D}_{q, \bar{p}}=\left(\mathscr{D}_{p, \bar{q}}\right)_{\mathscr{I}}^{\prime}, \forall p, q \in \mathscr{M}(r) \text { para algum } r \text { e } p \ll \mathscr{q},
$$

e, como conseqüência disto,

2.

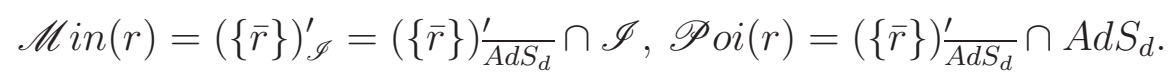

3. A bijeção

$$
\rho_{A d S_{d}}: \mathscr{W}_{p, q} \mapsto \rho_{A d S_{d}}\left(\mathscr{W}_{p, q}\right) \doteq \mathscr{D}_{p, q}, p, q \in \mathscr{M} i n(r) \text { para algum } r \text { e } p \ll \mathscr{q} q,
$$

denominada bijeção de REHREN, preserva complementos causais.

4. A ação de $\widetilde{S O}_{e}(2, d-1)$ sobre $\mathscr{I}$ é transitiva e preserva a estrutura causal. Portanto, age transitivamente sobre o conjunto dos diamantes. Como $S O_{e}(2, d-1)$ é o grupo conforme do espaço-tempo de Minkowski, segue que este age transitivamente sobre a coleção dos diamantes contidos em algum domínio de Minkowski.

5. Como $\widetilde{S O}_{e}(2, d-1)$ também preserva a estrutura causal de $A d S_{d}$, segue também que este grupo age transitivamente sobre o conjunto de cunhas.

6. Todo elemento de $\widetilde{S O}_{e}(2, d-1)$ pode ser obtido a partir de uma seqüência finita de isometrias da forma (1.26) para uma família adequada de diamantes / cunhas dentro do caso 3 da Observação 1.3, página 12.

A construção acima, que faz uso apenas das relações causais no fecho conforme, constitui o arcabouço geométrico da dualidade de REHREN [Reh00], e foi empregada nesta forma independente de coordenadas por Bousso e RANDALL [BR02] para estudar aspectos qualitativos da correspondência AdS/CFT.

\subsection{Espaços-tempos AAdS}

Tendo escrutinado com suficiente detalhe a estrutura de $A d S_{d}$, vamos preceder agora com o caso geral de nosso interesse. Seguindo a nomenclatura do Apêndice A, considere espaços-tempos estavelmente causais $(\mathscr{M}, g)$ dotados de completamento conforme $(\overline{\mathscr{M}}, \bar{g})$, infinito conforme $\left(\mathscr{I}, \bar{g}^{(0)}\right)$ e fator conforme $z$ satisfazendo as condições da Definição A.3 (página 119). 
DEFINIÇÃO 1.4 Dizemos que $(\mathscr{M}, g)$ é um espaço-tempo tipo AdS se $\left(\mathscr{I}, \bar{g}^{(0)}\right)$ é uma variedade Lorentziana $(d-1)$-dimensional (ou, equivalentemente, o covetor normal a $\mathscr{I},\left.d z\right|_{z=0}$, é tipo espaço com respeito a $\bar{g}$ ) e difeomorfo a $\mathbb{R} \times S^{d-2}$. Se, além disso, $\bar{g}^{(0)}$ pertencer à classe conforme da métrica de $E S U_{d-1},(\mathscr{M}, g)$ é dito ser assintoticamente AdS $(A A d S)$.

Finalmente, um espaço-tempo $(\mathscr{M}, g)$ dotado de infinito conforme $\left(\mathscr{I}, \bar{g}^{(0)}\right)$ é dito localmente tipo AdS (resp. localmente AAdS) se todo $p \in \mathscr{I}$ possui uma vizinhança aberta $\overline{\mathscr{U}}$ no completamento conforme $(\overline{\mathscr{M}}, \bar{g})$ tal que $(\overline{\mathscr{U}}, \bar{g}\lceil\overline{\mathscr{U}})$ é isométrica a uma vizinhança $\overline{\mathscr{V}}$ de $q \in \mathscr{J}$, onde $\mathscr{J}$ é o infinito conforme de um espaço-tempo tipo AdS (resp. AAdS).

A definição de espaços-tempos localmente tipo AdS e localmente AAdS engloba os exemplos de buracos negros com constante cosmológica negativa (SCHWARZSCHILDAdS, REISsner-NordströM-AdS, KerR-NEwMAN-AdS, etc.), não contemplados pela definição de espaços-tempos AAdS e tipo AdS.

Com a Definição 1.4 em mãos, definimos em espaços-tempos AAdS o antípoda $\bar{p}$ e o domínio de Minkowski $\mathscr{M} i n(p)$ ao futuro de $p \in \mathscr{I}$ exatamente como no caso AdS, uma vez que essas definições não se referem à geometria do interior. Mais em geral, temos a

Definição 1.5 Seja $(\mathscr{M}, g)$ um espaço-tempo localmente tipo AdS com infinito conforme $\left(\mathscr{I}, \bar{g}^{(0)}\right)$, e $p \ll \mathscr{I} q \in \mathscr{I}$ tais que $I^{+}(p, \overline{\mathscr{M}}) \cap I^{-}(q, \overline{\mathscr{M}})$ é relativamente compacto (portanto, globalmente hiperbólico) e $I^{+}(p, \mathscr{I}) \cap I^{-}(q, \mathscr{I})$ possui superfícies de CAUCHY contráteis. Denotemos a coleção dos pares $(p, q) \in \mathscr{I}^{2}$ satisfazendo estas condições por $\mathscr{D}(\mathscr{I})$.

$A$ cunha (no interior) associada a p, q é a região

$$
\mathscr{W}_{p, q}=I^{+}(p, \overline{\mathscr{M}}) \cap I^{-}(q, \overline{\mathscr{M}}) \cap \mathscr{M},
$$

e o diamante (na fronteira) associado a $p, q$, a região

$$
\mathscr{D}_{p, q}=I^{+}(p, \mathscr{I}) \cap I^{-}(q, \mathscr{I}) .
$$

$O$ horizonte passado $\partial_{-} \mathscr{W}_{p, q}$ (resp. horizonte futuro $\left.\partial_{+} \mathscr{W}_{p, q}\right)$ de $\mathscr{W}_{p, q}$ é dado por

$\partial_{-} \mathscr{W}_{p, q} \doteq\left(\partial I^{+}(p, \overline{\mathscr{M}}) \backslash\{p\}\right) \cap I^{-}(q, \overline{\mathscr{M}}) \cap \mathscr{M}, \partial_{+} \mathscr{W}_{p, q} \doteq\left(\partial I^{-}(q, \overline{\mathscr{M}}) \backslash\{q\}\right) \cap I^{+}(p, \overline{\mathscr{M}}) \cap \mathscr{M}$,

e o horizonte passado $\partial_{-} \mathscr{D}_{p, q}\left(\right.$ resp. horizonte futuro $\left.\partial_{+} \mathscr{D}_{p, q}\right)$ de $\mathscr{D}_{p, q}$, por

$$
\partial_{-} \mathscr{D}_{p, q} \doteq\left(\partial I^{+}(p, \mathscr{I}) \backslash\{p\}\right) \cap I^{-}(q, \mathscr{I}), \partial_{+} \mathscr{D}_{p, q} \doteq\left(\partial I^{-}(q, \mathscr{I}) \backslash\{q\}\right) \cap I^{+}(p, \mathscr{I}) .
$$


Denotamos por $\mathscr{W}(\mathscr{M}, g) \doteq\left\{\mathscr{W}_{p, q}:(p, q) \in \mathscr{D}(\mathscr{I})\right\}$ a coleção das cunhas em $(\mathscr{M}, g)$, e $\mathscr{D}(\mathscr{M}, g) \doteq\left\{\mathscr{D}_{p, q}:(p, q) \in \mathscr{D}(\mathscr{I})\right\}$, a coleção dos diamantes em $\left(\mathscr{I}, \bar{g}^{(0)}\right)$. A bijeção de ReHRen associada a $(\mathscr{M}, g)$ é dada por

$$
\begin{aligned}
\rho_{(\mathscr{M}, g)}: \mathscr{W}(\mathscr{M}, g) & \longrightarrow \mathscr{D}(\mathscr{M}, g) \\
\mathscr{W}_{p, q} & \mapsto \rho_{(\mathscr{M}, g)}\left(\mathscr{W}_{p, q}\right) \doteq \mathscr{D}_{p, q} .
\end{aligned}
$$

Note que é a Observação 1.3 que nos permitiu estender naturalmente a definição de cunhas e diamantes para espaços-tempos localmente tipo AdS. Se $(\mathscr{M}, g)$ é um espaçotempo AAdS, o domínio de Poincaré ao futuro de $r \in \mathscr{I}$ coincide, assim como no caso de domínios de Minkowski, com a definição no caso AdS: $\mathscr{P}_{o i}(r) \doteq \mathscr{W}_{r, \overline{\bar{r}}}$.

As cunhas num espaço-tempo localmente tipo AdS recobrem precisamente a região

$$
I^{-}(\mathscr{I}, \overline{\mathscr{M}}) \cap I^{+}(\mathscr{I}, \overline{\mathscr{M}}) \cap \mathscr{M} \doteq \mathscr{W}(\mathscr{I})
$$

denominada dominio de comunicação exterior de $(\mathscr{M}, g)$, que é identificada em vários exemplos com o exterior de buracos negros AAdS.

Nas Subseção 1.3.1 abaixo, estudaremos as propriedades causais, topológicas e de localização de $\rho_{(\mathscr{M}, g)}$, em termos das geodésicas nulas dos espaços-tempos AAdS.

\subsubsection{Geometria global e geodésicas nulas}

\subsubsection{Causalidade (interior para fronteira)}

O universo estático de Einstein $\mathscr{I}$ é globalmente hiperbólico, e as órbitas geradas pelo subgrupo de $\widetilde{S O}_{e}(2, d-1)$ correspondente à translação temporal em $A d S_{d}$ (rotação dos pontos da hiperquádrica (1.5), página 5 , no plano $X^{0}-X^{d}$ ) correspondem às linhas paralelas ao cilindro ${ }^{5} \mathbb{R} \times S^{d-2}$, constituíndo geodésicas de tipo tempo que maximizam o tempo próprio entre seus pontos. Mais ainda, todo ponto em $\mathscr{I}$ pertence a uma única geodésica desta família, e podemos escolher uma parametrização comum a todas essas geodésicas (denominadas geradores temporais de $\mathscr{I}$ ) tal que a união dos pontos correspondentes a um mesmo valor do parâmetro afim constituem uma superfície de CAUCHY.

Com isso, podemos definir o atraso temporal (gravitacional) de uma geodésica nula completa $\gamma$ em $\mathscr{M}$, com extremos em $\mathscr{I}$ : grosso modo, ele representa o quanto que o extremo futuro de $\gamma$ se afasta do antípoda do extremo passado de $\gamma$, que no caso de

\footnotetext{
${ }^{5} \mathrm{O}$ gerador desse grupo corresponde a $1 / 2\left(P^{0}+K^{0}\right)$ num domínio de Minkowski, onde $P^{0}$ é o gerador de translações na direção $x^{0}$, e $K^{0}$ o gerador de transformações conformes especiais na direção $x^{0}$.
} 
AdS, é o extremo futuro.

Primeiro, vamos provar que, dadas certas condições extras, o atraso temporal gravitacional de geodésicas nulas inextensíveis é sempre positivo (ver Figura 1.3 para ilustração do resultado):

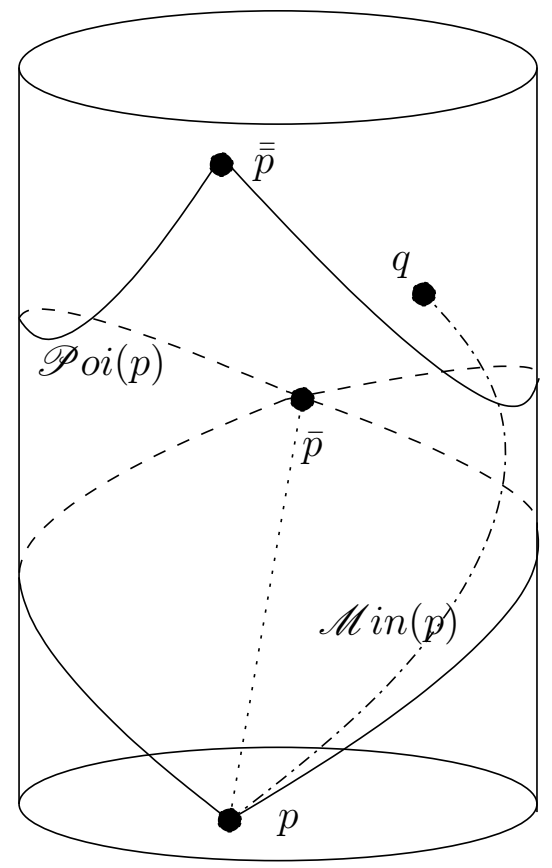

Figura 1.3: Atraso temporal gravitacional. As geodésicas nulas que emanam de $p \in \mathscr{I}$ e permanecem em $\mathscr{I}$ (linhas cheias) são focadas no antípoda $\bar{p}$. Enquanto que uma geodésica nula emanando de $p$ e atravessando o interior também teria extremo futuro em $\bar{p}$ em $A d S_{d}$ (linha pontilhada), num espaço-tempo AAdS satisfazendo a hipótese de focalização global do Teorema 1.3 tal geodésica tem extremo futuro em $q \in I^{+}(p, \mathscr{I})$ (linha alternando pontos e traços).

Teorema 1.3 (atraso temporal gravitacional positivo) Seja $(\mathscr{M}, g)$ um espaço-tempo AAdS satisfazendo a seguinte hipótese de focalização global: toda geodésica nula inextensivel possui um par de pontos conjugados (ver no Apêndice A a discussão que segue a equação (A.14), página 112). Então, dado $p \in \mathscr{I}$, toda geodésica nula inextensivel em $\mathscr{M}$ com extremo passado em p possui, se houver, extremo futuro em $I^{+}(p, \mathscr{I})$. O mesmo resultado vale se trocarmos o futuro pelo passado, e $I^{+}(p, \mathscr{I})$ por $I^{-}(p, \mathscr{I})$.

Prova. Inicialmente, provaremos dois Lemas: 
Lema 1.4 (Ausência de atalhos Causais) Sejam $p, p^{\prime} \in \mathscr{I}$. Se $p \perp_{\mathscr{I}} p^{\prime}$, entẫo não pode existir uma curva causal em $(\overline{\mathscr{M}}, \bar{g})$ ligando $p$ a $p^{\prime}$.

Prova. Suponha que $p^{\prime}>\overline{\mathscr{M}} p$ (o caso oposto é tratado de forma similar). Provaremos que o atraso temporal gravitacional implicado pelas hipóteses do Teorema contradiz a disjunção causal de $p$ e $p^{\prime}$ em relação a $\mathscr{I}$. Denote por $T\left(p^{\prime}\right)$ o único gerador temporal de $\left(\mathscr{I}, \bar{g}^{(0)}\right)$ contendo $p^{\prime}$.

Note que $\partial I^{+}(p, \mathscr{I}) \doteq \Sigma$ é uma superfície fechada e acronal, que corta $\left(\mathscr{I}, \bar{g}^{(0)}\right)$ em dois abertos disjuntos $I^{+}(p, \mathscr{I}) \doteq A$ e $\mathscr{I} \backslash \overline{I^{+}(p, \mathscr{I})} \doteq B$, e intersecta cada gerador temporal de $\mathscr{I}$ precisamente uma vez, já que todo gerador temporal possui pontos em $I^{+}(p, \mathscr{I})$ e $I^{-}(\bar{p}, \mathscr{I})$. Por hipótese, $p^{\prime} \in B$. Mais ainda, $T\left(p^{\prime}\right)$ deve cruzar $\Sigma$ em algum instante. Portanto, existe um $p^{\prime \prime} \in T\left(p^{\prime}\right)$ tal que $p^{\prime \prime} \gg \mathscr{I} p^{\prime}$ e $p^{\prime \prime} \in \Sigma$. Seja $\eta$ o gerador nulo de $\Sigma$ que contém $p^{\prime \prime}$. Como o segmento de $\eta$ que liga $p$ a $p^{\prime \prime}$ é nulo e acronal, $\eta$ é necessariamente a curva mais rápida em $\left(\mathscr{I}, \bar{g}^{(0)}\right)$ ligando $p$ a $T\left(p^{\prime}\right)$.

Agora, considera a fronteira acronal $\partial I^{+}(p, \overline{\mathscr{M}})=\partial J^{+}(p, \overline{\mathscr{M}}) \doteq \bar{\Sigma}$. $\bar{\Sigma} \cap \mathscr{I}$ é fechado, acronal e intersecta cada gerador temporal de $\mathscr{I}$ em precisamente um ponto, pois todo gerador temporal possui pontos em $I^{+}(p, \overline{\mathscr{M}})$ e $I^{-}(p, \overline{\mathscr{M}})$, e $\bar{\Sigma}$ separa $\overline{\mathscr{M}}$ em dois abertos disjuntos (do ponto de vista de uma variedade com bordo, é claro $) I^{+}(p, \overline{\mathscr{M}}) \doteq \dot{A} \mathrm{e}$ $\overline{\mathscr{M}} \backslash \overline{I^{+}(p, \overline{\mathscr{M}})} \doteq \dot{B}$. Portanto, $T\left(p^{\prime}\right)$ precisa cruzar $\bar{\Sigma}$ em, digamos, $p^{\prime \prime \prime}$. Uma vez que $p<p^{\prime}$ do ponto de vista de $(\overline{\mathscr{M}}, \bar{g})$, devemos ter $p^{\prime \prime \prime} \ll \mathscr{I} p^{\prime}$ ou $p^{\prime \prime \prime}=p^{\prime}$. Em ambos os casos, temos $p^{\prime \prime \prime} \ll \mathscr{I} p^{\prime \prime}$, o que implica que o gerador nulo $\bar{\eta}$ de $\bar{\Sigma}$ contendo $p^{\prime \prime \prime}$ é estritamente mais rápido que $\eta$. Como $\eta$ era a curva mais rápida em $\left(\mathscr{I}, \bar{g}^{(0)}\right)$ ligando $p$ a $T\left(p^{\prime}\right), \bar{\eta}$ necessariamente atravessa $\mathscr{M}$, o que contradiz as hipóteses do Teorema, pois $\bar{\eta}$ deve ser necessariamente acronal e, portanto, não pode ter um par de pontos conjugados [BEE96, HE73, Wal84].

Lema 1.5 Seja $p, p^{\prime} \in \mathscr{I}$. Se $p^{\prime} \in \partial I^{+}(p, \mathscr{I})$ e $p^{\prime} \neq \bar{p}$, então não pode existir um segmento geodésico nulo em $(\overline{\mathscr{M}}, \bar{g})$ que não pertença a $\mathscr{I}$ ligando $p$ a $p^{\prime}$.

Prova. $\quad$ Seja $\eta$ o (necessariamente único) gerador nulo de $\partial I^{+}(p, \mathscr{I})$ ligando $p$ a $p^{\prime}$. Suponha que exista outra geodésica nula $\bar{\eta}$ atravessando mathscr $M$ e ligando $p$ a $p^{\prime}$. Como $\left(\mathscr{I}, \bar{g}^{(0)}\right)$ é totalmente geodésica por construção, segue que $\bar{\eta}$ necessariamente incide em $p^{\prime}$ transversamente a $\mathscr{I}$. Então, se escolhermos qualquer ponto $p^{\prime \prime}$ em $\eta$ depois de $p^{\prime}$, então existe um segmento geodésico nulo futuro e quebrado ligando $p$ a $p^{\prime \prime}$, o que implica, por sua vez, que existe uma curva de tipo tempo em $(\overline{\mathscr{M}}, \bar{g})$ ligando $p$ a $p^{\prime \prime}$ [HE73].

Seja $T\left(p^{\prime \prime}\right)$ o gerador temporal de $\left(\mathscr{I}, \bar{g}^{(0)}\right)$ contendo $p^{\prime \prime}$. Agora, considere $\bar{\Sigma}$ como no Lema anterior. Novamente, $T\left(p^{\prime \prime}\right)$ deve cruzar $\bar{\Sigma}$, 
digamos em $p^{\prime \prime \prime}$. Então, necessariamente $p^{\prime \prime \prime} \ll \mathscr{I} p^{\prime \prime}$. Mas isto implica que, dado que $(\overline{\mathscr{M}}, \bar{g})$ é (fortemente) causal, $p \perp \mathscr{I} p^{\prime}$. Isto contradiz o resultado do Lema 2.1 .

Retornando à demonstração do Teorema, seja $\gamma$ um segmento geodésico nulo emanando de $p$, atravessando $\mathscr{M}$ e com extremo futuro $p^{\prime} \in \mathscr{I}$. Como assumimos que $(\mathscr{M}, g)$ é (fortemente) causal, vê-se que $p^{\prime} \notin \overline{I^{-}(p, \overline{\mathscr{M}})}$. Os Lemas 1.4 e 1.5 implicam que, se $p^{\prime}$ não é arrastado para dentro de $I^{+}(p, \mathscr{I})$ por atraso temporal gravitacional, então $p^{\prime}=\bar{p}$, exatamente como no caso de $A d S_{d}$. Mas, mesmo neste caso, a presença de um par de pontos conjugados em $\gamma$ implica que existe uma curva tipo tempo atravessando o interior e ligando $p$ a $p^{\prime}=\bar{p}$. Repetindo o argumento do Lema 1.5, o resultado segue. A demonstração para o caso com orientação temporal reversa é a mesma.

OBSERVAÇÃo 1.6 O método usado na demonstração é similar à demonstração do teorema de massa positiva para espaços-tempos assintoticamente planos demonstrado por Penrose, Sorkin e Woolgar [PSW93], e para espaços-tempos AAdS por WoolGAR [Woo94] e PAGE, SuRYa e WoOlgAR [PSW02] - uma discussão breve acerca do resultado de [PSW02] terá lugar no Capítulo 2. Uma prova de resultado similar ao Teorema 1.3, empregando uma estratégia diferente, foi proposta por GAO e WALD [GW00].

A condição expressa no Teorema 1.3 acerca da focalização global de geodésicas nulas exerce um papel crucial na sua demonstração, pois a onipresença de pares de pontos conjugados exclui a presença de linhas nulas, i.e., geodésicas nulas completas e acronais. Isto vem do fato de que, dado um par de pontos conjugados $p<q$ numa geodésica nula $\gamma$, qualquer ponto $r>q$, resp. $r<p$ satisfaz $p \ll r$, resp. $q \gg r$. Mais precisamente, a prova do Teorema 1.3 consiste em mostrar que, em todas as instâncias possíveis de violação da assertiva, é possível construir uma linha nula atravessando o interior. Tal situação contrasta nitidamente com o caso de $A d S_{d}$, uma vez que, neste último, todas as geodésicas nulas emanando de $p$ e atravessando o interior se encontram em $\bar{p}$.

Esta condição de focalização é válida se, por exemplo, as equações de Einstein sem matéria (1.1) são satisfeitas em toda parte, pois estas implicam na validade da condição de energia nula $\operatorname{Ric}(g)_{a b} k^{a} k^{b} \geq 0$ para todo $k^{a}$ tipo luz, $e$ se a chamada condição genérica nula é satisfeita em todas as geodésicas nulas inextensíveis (ver [BEE96] para uma discussão acerca dessa condição). Esta última condição é necessária para que a expansão de $\partial I^{+}(p, \overline{\mathscr{M}}) \cap \mathscr{M}$ eventualmente se torne negativa em cada um de seus geradores - a equação de RAYCHAUDHURi (A.7) e a condição de energia nula se encarregam então de mostrar que existem dois pontos conjugados na extensão maximal de cada gerador (ver no Apêndice A a discussão seguindo as fórmulas (A.11), página 
112, e (A.23), página 115).

A violação da condição genérica nula sugere uma certa rigidez geométrica para hipersuperfícies nulas com tal propriedade. Que esta intuição é justificada em nível global, pode ser visto pelos seguintes resultados:

Teorema 1.6 (Teorema Geométrico do Máximo [AGH98a, Gal00]) Seja $\mathscr{S}_{1}$ uma hipersuperfície tipo luz futura (i.e., cujos geradores nulos (futuros) são inextensíveis no futuro) e $\mathscr{S}_{2}$ uma hipersuperfície tipo luz passada (i.e., cujos geradores nulos (passados) são inextensíveis no passado). Suponha que:

1. $\mathscr{S}_{1}$ e $\mathscr{S}_{2}$ possuem um ponto comum $p \in \mathscr{M}$ e $\mathscr{S}_{2}$ encontra-se no lado futuro de $\mathscr{S}_{1}$ numa vizinhança de $p$;

2. $\mathscr{S}_{1}$ tem expansão nula $\theta_{1}>0$ (ver no Apêndice $A$ a discussão precedendo imediatamente a fórmula (A.9), página 111) no sentido de suporte ${ }^{6}$ tal que o conjunto das segundas formas fundamentais nulas das hipersuperfícies de suporte $\mathscr{S}_{, \epsilon}$ é limitado por baixo;

3. $\mathscr{S}_{2}$ possui expansão $\theta_{2} \leq 0$ no sentido de suporte,

então $\mathscr{S}_{1}$ e $\mathscr{S}_{2}$ coincidem numa vizinhança $\mathscr{O}$ de $p$. Mais ainda, $\mathscr{S}_{1} \cap \mathscr{O}=\mathscr{S}_{2} \cap \mathscr{O}$ é uma hipersuperficie $\mathscr{C}^{\infty}$ tipo luz com expansão $\theta=0$.

A prova do Teorema 1.6 consiste em denotar $\mathscr{S}_{1}$ e $\mathscr{S}_{2}$ localmente por um gráfico de uma função Lipschitziana, e, nessas coordenadas, reescrever a expansão nula como um operador elíptico quase-linear de segunda ordem, similar ao operador de curvatura média prescrita numa subvariedade Riemanniana de codimensão 1, para o qual existem princípios de máximo (ver, por exemplo, [AGH98a, GT98]). Do Teorema 1.6 segue o

Teorema 1.7 (Teorema da Cisão Nula [Gal00]) Seja $(\mathscr{M}, g)$ um espaço-tempo dotado de completeza geodésica nula e com tensor de RICCI satisfazendo a condição de energia nula em toda a parte. Se $\mathscr{M}$ possui uma linha nula $\gamma$, então $\gamma$ pertence a uma

\footnotetext{
${ }^{6}$ Dada uma hipersuperfície $\mathscr{C}^{0}$ tipo luz futura $\mathscr{S}$ e $p \in \mathscr{S}$, uma hipersuperfície de suporte futuro (resp. passado) para $\mathscr{S}$ em $p$ é uma hipersuperfície $\mathscr{C}^{\infty}$ futura (resp. passada) $\mathscr{S}_{p}$ que tem um segmento geodésico nulo, contendo $p$ em seu interior, em comum com $\mathscr{S}$, localizada no lado futuro (resp. passado) de $\mathscr{S}$ numa vizinhança de $p$. Definimos analogamente hipersuperfícies de suporte para hipersuperfícies $\mathscr{C}^{0}$ tipo luz passadas. Dizemos que uma hipersuperfície $\mathscr{C}^{0}$ tipo luz $\mathscr{S}$ futura (resp. passada) possui expansão $\theta \geq 0$ (resp. $\leq 0$ ) no sentido de suporte se, para cada $p \in \mathscr{S}, \epsilon>0$, existe uma hipersuperfície de suporte passado (resp. futuro) $\mathscr{S}_{p, \epsilon}$ para $\mathscr{S}$ em $p$ tal que a expansão $\theta_{p, \epsilon}$ de $\mathscr{S}_{p, \epsilon}$ satisfaz $\theta_{p, \epsilon}(p) \geq-\epsilon$ (resp. $\left.\leq+\epsilon\right)$. Hipersuperfícies de suporte constituem uma maneira de estudar a expansão de hipersuperfícies nulas não-diferenciáveis devido à presença de cáusticas, como por exemplo fronteiras acronais, horizontes de buracos negros, etc..
} 
hipersuperfície nula, acronal, sem bordo e totalmente geodésica, i.e., qualquer geodésica tangente à hipersuperfície em qualquer ponto pertence a esta última numa vizinhança deste ponto.

Retornando ao nosso estudo, outra diferença fundamental entre espaços-tempos AdS e espaços-tempos AAdS "genéricos" está expressa na seguinte Proposição, que em particular implica que a coleção das cunhas em um espaço-tempo AAdS pode não ser fechada por complementos causais, embora ainda haja preservação de causalidade pela bijeção de REHREN neste caso. Mais precisamente, temos que $\mathscr{W}_{p, \bar{q}}^{\prime} \cap \mathscr{W}_{q, \bar{p}}^{\prime} \neq \varnothing$ (ver Figura 1.4), em virtude da

Proposição 1.8 (Segunda Lei da Dinâmica de Cunhas AAdS) Seja $(\mathscr{M}, g)$ um espaço-tempo AAdS satisfazendo a condição de energia nula Ric $(g) k^{a} k^{b} \geq 0$ para todo vetor tipo luz $k^{a}$ e as condições do Teorema 1.3. Defina $\Xi_{p}^{+} \doteq \partial I^{+}(p, \overline{\mathscr{M}}) \backslash\{p, \bar{p}\}$ e $\Xi_{\bar{p}}^{-} \doteq \partial I^{-}(\bar{p}, \overline{\mathscr{M}}) \backslash\{p, \bar{p}\}$. Ent $\tilde{a} o:$

(i) $\Xi_{p}^{+} \cap I^{-}(\bar{p}, \overline{\mathscr{M}})=\Xi_{\bar{p}}^{-} \cap I^{+}(p, \overline{\mathscr{M}})=\varnothing$.

(ii) $\Xi_{p}^{+} \cap \Xi_{\bar{p}}^{-} \cap \mathscr{M}=\varnothing$.

Prova. (i) Sabemos que $\Xi_{p}^{+} \cap \mathscr{I}=\Xi_{\bar{p}}^{-} \cap \mathscr{I}$, então vamos nos concentrar apenas no interior. A saber, suponha que existe $q \in \mathscr{M}$ tal que $q \in \Xi_{\bar{p}}^{-}$e $q \notin \Xi_{p}^{+}$. Se $q \gg p$, isso contradiz o fato de que não existe uma curva de tipo tempo ligando $p$ a $\bar{p}$. Repetir o argumento trocando passado pelo futuro, e os papéis de $p$ e $\bar{p}$.

(ii) Suponha que $\Xi_{p}^{+}$e $\Xi_{\bar{p}}^{-}$coincidem em algum ponto $q \in \mathscr{M}$. Sabemos que (a) $\mathscr{S}_{1} \doteq \Xi_{\bar{p}}^{-} \cap \mathscr{M}$ é uma hipersuperfície $\mathscr{C}^{0}$ nula futura, e $\mathscr{S}_{2} \doteq \Xi_{p}^{+}$é uma hipersuperficie $\mathscr{C}^{0}$ nula passada. Tomando quaisquer $q_{2} \leq q \leq q_{1} \operatorname{com} q_{i} \in \mathscr{S}_{i}$, $i=1,2$, vemos que $\partial I^{-}\left(q_{1}, \mathscr{M}\right)$ contém uma hipersuperfície de suporte passado $\mathscr{S}_{-}$para $\mathscr{S}_{1}$ em $q$ e $\partial I^{+}\left(q_{2}, \mathscr{M}\right)$, uma hipersuperfície de suporte futuro $\mathscr{S}_{+}$para $\mathscr{S}_{2}$ em $q$. Usando a hipótese de que $\operatorname{Ric}(g)_{a b} k^{a} k^{b} \geq 0$ para todo vetor tipo luz $k^{a}$, podemos invocar a equação de RAYChaudhuri (A.7) (página 110) e, lembrando que as congruências de geodésicas nulas dadas por $\mathscr{S}_{-}$e $\mathscr{S}_{+}$têm torção $\omega_{+a b}=\omega_{-a b}=0$, mostrar que $-\frac{1}{\theta_{-}^{2}} \frac{d \theta_{-}}{d \lambda_{-}} \leq-\frac{1}{d-2}$ e $-\frac{1}{\theta_{+}^{2}} \frac{d \theta_{+}}{d \lambda_{+}} \geq+\frac{1}{d-2}$, onde $\lambda_{ \pm}$é uma parametrização afim das geodésicas nulas $\gamma_{ \pm}$respectivamente em $\mathscr{S}_{2}$ e $\mathscr{S}_{1}$ tal que $\gamma_{ \pm}(0)=q, \gamma_{-}(\lambda)=q_{2}$ e $\gamma_{+}(\lambda)=q_{1}$, para algum $\lambda>0$. Daí segue que $\theta_{-}(p) \geq-\frac{d-2}{\lambda}$ e $\theta_{+}(p) \leq \frac{d-2}{\lambda}$. Tomando $q_{2}$ suficientemente próximo de $p$ e $q_{1}$ suficientemente próximo de $\bar{p}$, podemos tomar $\lambda$ arbitrariamente grande. Logo, (b) $\mathscr{S}_{1}$ e $\mathscr{S}_{2}$ satisfazem $\theta_{2} \leq 0 \leq \theta_{1}$ no sentido de suporte. Concluímos, assim, de (a), (i) e (b) que $\mathscr{S}_{1}$ e $\mathscr{S}_{2}$ satisfazem as hipóteses do Teorema 1.6 e, portanto, ambas coincidem numa vizinhança de $q$, onde formam uma hipersuperfície $\mathscr{C}^{\infty}$ tipo luz com expansão zero. Isso implica que $\Xi_{p}^{+}$e $\Xi_{\bar{p}}^{-}$devem coincidir ao longo de uma geodésica nula contendo $q$ e portanto atravessando o interior. Neste caso, $\gamma$ 
deve possuir $p$ como extremo passado e $\bar{p}$ como extremo futuro. Mas essa geodésica pertence a uma fronteira acronal, e portanto deve ser acronal, contradizendo as hipóteses do Teorema 1.3.

(a)

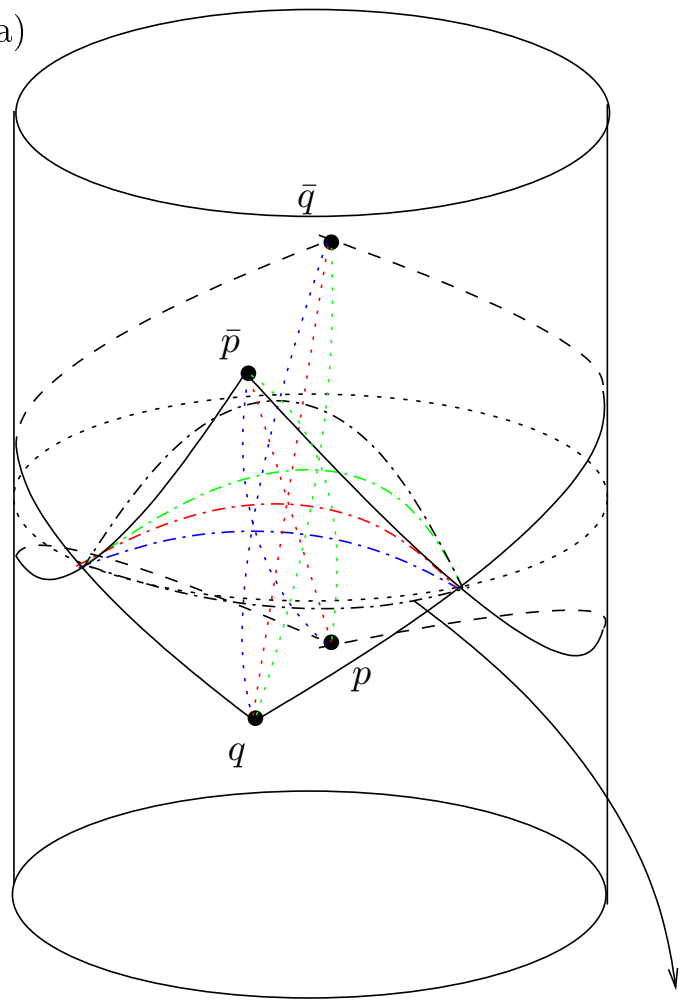

(b)

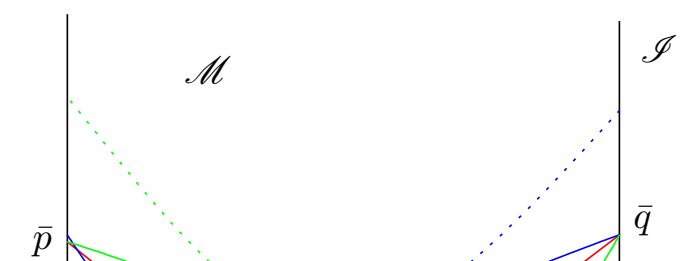

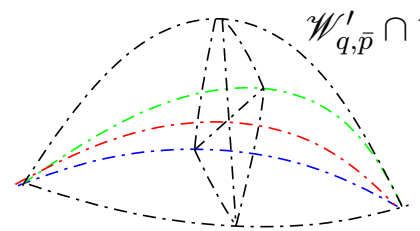

Figura 1.4: Efeito do atraso temporal gravitacional (cujo efeito sobre geodésicas nulas é exemplificado pelas linhas pontilhadas no corte longitudinal (b) de (a)) sobre cunhas e seus complementos causais em espaços-tempos AAdS. Enquanto que, em $A d S_{d}$, o complemento causal da cunha $\mathscr{W}_{p, \bar{q}}$ é a cunha $\mathscr{W}_{q, \bar{p}}$ (vermelho), os complementos causais de ambas as cunhas (respectivamente verde e azul) não mais o são em espaços-tempos AAdS satisfazendo as condições da Proposição 1.8 e, portanto, do Teorema 1.3, e de uma maneira tal que a região aberta $\mathscr{W}_{p, \bar{q}}^{\prime} \cap \mathscr{W}_{q, \bar{p}}^{\prime} \neq \varnothing$ é não-vazia, o que não pode ocorrer em $A d S_{d}$. 
OBSERVAÇÃo 1.7 Note que, antes de invocarmos a hipótese de focalização global do Teorema 1.3 na demonstração de (ii), vimos que, novamente, foi produzida uma linha nula $\gamma$ contida em $\Xi_{p}^{+}$e $\Xi_{\bar{p}}^{-}$. No momento em que invocamos o Teorema 1.6, se $(\mathscr{M}, g)$ fosse assintoticamente simples, poderíamos ter ido mais longe: pelo Teorema de Cisão Nula 1.7, a hipersuperfície tipo luz que contém a linha nula em $\mathscr{M}$ ligando $p$ a $\bar{p}$ não possui bordo, o qual seria constituido de pontos extremais de $\partial I^{+}(p, \mathscr{M})$ e $\partial I^{-}(\bar{p}, \mathscr{M})$ caso existisse. Portanto, segue da completeza geodésica nula de $(\mathscr{M}, g)$ que $\partial I^{+}(p, \mathscr{M})=\partial I^{-}(\bar{p}, \mathscr{M})$ é $\mathscr{C}^{\infty}$ e totalmente geodésica em relação a $\mathrm{g}$.

Uma conseqüência importante da Proposição 1.8 é que a condição de focalização causa um "encolhimento" não-trivial das cunhas de AAdS rumo ao infinito conforme, como podemos ver na Figura 1.4 - ver a discussão no final do Capítulo 2 para uma interpretação dinâmica deste fenômeno, que explica o porquê de denominarmos esta Proposição Segunda Lei da Dinâmica de Cunhas AAdS.

\subsubsection{Conexidade simples (interior versus fronteira)}

Citaremos agora, por completeza, dois resultados que mostram que toda cunha $\mathscr{W}_{p, q} \in \mathscr{W}(\mathscr{M}, g)$ num espaço-tempo localmente tipo AdS $(\mathscr{M}, g)$, satisfazendo certas condições adicionais, é simplesmente conexa se $\mathscr{D}_{p, q}$ o for (por exemplo, cunhas contidas em um domínio de PoINCARÉ de um espaço-tempo AAdS). Mais precisamente, toda curva causal no interior com extremos em $p$ e $q$ é homotópica a uma curva causal em $\mathscr{I}$ ligando $p$ a $q$. Em suma, a topologia de $\mathscr{W}_{p, q}$ é determinada pela topologia de $\mathscr{D}_{p, q}$, mostrando que toda cunha $\mathscr{W}_{p, q} \in \mathscr{W}(\mathscr{M}, g)$ é contrátil.

Este é um caso particular de censura topológica - a topologia da região exterior ao horizonte de eventos de um buraco negro é determinada pela topologia de $\mathscr{I}$ demonstrada no presente contexto por Galloway, Schleich, WitT e Woolgar [GSWW99].

Teorema 1.9 (Galloway, Schleich, Witt e Woolgar [GSWW99]) Seja um espaço-tempo localmente tipo $A d S(\mathscr{M}, g)$ tal que $(\overline{\mathscr{M}}, \bar{g})$ é globalmente hiperbólico e $\mathscr{I}$ possui uma componente conexa $\mathscr{I}_{0}$ com um corte tipo espaço compacto. ${ }^{7}$ Suponha que toda geodésica nula completa no futuro $\gamma$ em $(\overline{\mathscr{M}}, \bar{g})$ emanando de algum $p=\gamma(0)$ numa vizinhança de $\mathscr{I}_{0}$ satisfaz $\int_{0}^{+\infty} \operatorname{Ric}(\bar{g}(\gamma(\lambda)))_{a b} \dot{\gamma}^{a}(\lambda) \dot{\gamma}^{b}(\lambda) d \lambda \geq 0$. Então, não existe comunicação causal futura de $\mathscr{I}_{0}$ através de $\mathscr{M}$ com nenhuma outra componente de $\mathscr{I}$, i.e., $J^{+}\left(\mathscr{I}_{0}, \overline{\mathscr{M}}\right) \cap\left(\mathscr{I} \backslash \mathscr{I}_{0}\right)=\varnothing$.

O Teorema 1.9 complementa esplendidamente o Lema 1.4, empregado na demonstração do Teorema 1.3. Empregando-se o Teorema 1.9 e argumentos de espaços de

\footnotetext{
${ }^{7}$ Dado uma variedade (conexa) $\mathscr{M}$, um corte é uma hipersuperfície sem bordo $\Sigma$ tal que $\mathscr{M} \backslash \Sigma$ possui duas componentes conexas.
} 
recobrimento [GH81, O’N83], pode-se provar finalmente o seguinte teorema de censura topológica:

Teorema 1.10 (Galloway, Schleich, Witt e Woolgar [GSWW99]) Seja um espaço-tempo localmente tipo $\operatorname{Ad} S(\mathscr{M}, g)$ tal que $\left(\mathscr{W}(\mathscr{I}) \cup \mathscr{I},\left.\bar{g}\right|_{\mathscr{W}}(\mathscr{I}) \cup \mathscr{I}\right)$ é globalmente hiperbólico e $\mathscr{I}$ possui um corte tipo espaço compacto. Suponha que toda geodésica nula completa no futuro $\gamma$ em $(\overline{\mathscr{M}}, \bar{g})$ emanando de algum $p=\gamma(0)$ numa vizinhança de $\mathscr{I}$ satisfaz $\int_{0}^{+\infty} \operatorname{Ric}(\bar{g}(\gamma(\lambda)))_{a b} \dot{\gamma}^{a}(\lambda) \dot{\gamma}^{b}(\lambda) d \lambda \geq 0$. Ent $\tilde{a} o$ toda curva causal $\gamma:(0,1) \rightarrow$ $\mathscr{W}(\mathscr{I})$ com extremos $\gamma(0), \gamma(1) \in \mathscr{I}$ é homotópica com extremos fixos a uma curva $\gamma_{0}:[0,1] \rightarrow \mathscr{I}(\operatorname{ver}$ Apêndice $D)$.

Segue imediatamente do Teorema 1.10 a contratividade de $\mathscr{W}_{p, q}$ para $p, q \in \mathscr{D}(\mathscr{I})$. Em particular, se $p, q \in \mathscr{D}(\mathscr{I})$ são os extremos de $\gamma$ no Teorema 1.10, podemos escolher $\gamma_{0}$ cronológica.

\subsubsection{Localização (fronteira para interior)}

Ao longo desta Subsubseção, será assumido que $(\mathscr{M}, g)$ é um espaço-tempo AAdS assintoticamente simples satisfazendo as hipóteses do Teorema 1.3.

Conhecer a localização dos procedimentos físicos nas cunhas pode não ser suficiente para a reconstrução completa da teoria quântica no interior no contexto do Capítulo 4, empregando-se apenas informação da fronteira e a bijeção de $\operatorname{REHREN} \rho_{(\mathscr{M}, g)}$ introduzida pela Definição 1.5. Precisamos ser capazes de especificar a localização dos procedimentos físicos em abertos arbitrariamente pequenos, ou, o que dá no mesmo, sua localização com respeito a uma base para a topologia do interior. Isto pode ser obtido, em princípio, tomando-se intersecções de cunhas, mas não é nem um pouco claro se isto resulta numa base para a topologia ou não. Isto precisa ser feito de maneira mais precisa.

É sabido que um espaço-tempo cronológico, tal que toda geodésica nula inextensível possui um par de pontos conjugados, é fortemente causal [BEE96]. Para tais espaçostempos, a topologia gerada pelos diamantes (topologia de AlEXANDrov) coincide com a topologia da variedade subjacente. Portanto, em AdS, a questão acima é resolvida de forma positiva, pois qualquer diamante em $A d S$ (para o propósito de gerar a topologia da variedade, estes bastam) pode ser envolvida por cunhas em $A d S_{d}$ : dados

$$
\mathscr{O}_{p, q} \doteq I^{+}\left(p, A d S_{d}\right) \cap I^{-}\left(q, A d S_{d}\right), p \ll_{A d S_{d}} q,
$$

podemos escrever

$$
\mathscr{O}_{p, q}=\bigcap_{\substack{r \in \partial I^{-}\left(p, \overline{A d S_{d}}\right) \cap \mathscr{I}, s \in \partial I^{+}\left(q, \overline{A d S_{d}}\right), r, s \in \mathscr{M} \text { in }(u)}} \mathscr{W}_{r, s} .
$$


Veremos em breve que a acronalidade das geodésicas nulas inextensíveis em AdS é crucial para uma envoltória precisa. Já num espaço-tempo AAdS assintoticamente simples satisfazendo as hipóteses do Teorema 1.3, a questão é mais delicada, por causa das seguintes

Proposição 1.11 Seja $p \in \mathscr{M}$. Então, $\partial I^{+}(p, \overline{\mathscr{M}})$ intersecta cada gerador de tipo tempo de $\left(\mathscr{I}, \bar{g}^{(0)}\right)$ precisamente uma vez.

Prova. Pela acronalidade de $\partial I^{+}(p, \overline{\mathscr{M}})$, este intersecta cada gerador de tipo tempo de $\left(\mathscr{I}, \bar{g}^{(0)}\right)$ no máximo uma vez. Suponha, então, que a tese é falsa. Então, dado um gerador de tipo tempo $T$, temos as seguintes possibilidades:

(i) $T \subset I^{+}(p, \overline{\mathscr{M}})$ - Considere uma geodésica nula completa $\gamma$ cruzando $p$, e seja $q$ o extremo passado de $\gamma$. Então, existe um valor $t$ do parâmetro afim de $T$ tal que $T(t) \ll \mathscr{I} q$. Portanto, $T(t) \ll \overline{\mathscr{M}}_{p}$, o que é absurdo uma vez que $(\overline{\mathscr{M}}, \bar{g})$ é cronológico.

(ii) $T \cap J^{+}(p, \overline{\mathscr{M}})=\varnothing$ - Considere uma geodésica nula completa $\gamma$ cruzando $p$, e seja $r$ o extremo futuro de $\gamma$. Então, existe um valor $t$ do parâmetro afim de $T$ tal que $T(t) \gg \mathscr{I} r$. Portanto, $T(t) \gg_{\mathscr{M}} p$, contradizendo a hipótese.

Proposição 1.12 Sejam $q, r \in \mathscr{M}$ tal que $r \in \partial I^{-}(q, \mathscr{M})$, e $\gamma$ um gerador nulo de $\partial I^{-}(q, \mathscr{M})$ ao qual $r$ pertence. Sejam $s_{1}(r), s_{2}(r), s_{3}(r) \in \mathscr{I}$ definidos como segue:

- $s_{1}(r)$ é o extremo futuro de $\gamma$;

- $s_{2}(r)$ é o ponto onde $\partial I^{+}(q, \overline{\mathscr{M}})$ intersecta o gerador de tipo tempo de $T\left(s_{1}(r)\right)$ ao qual $s_{1}(r)$ pertence;

- $s_{3}(r)$ é o ponto onde $\partial I^{+}(r, \overline{\mathscr{M}})$ intersecta $T\left(s_{1}(r)\right)$.

\section{Então:}

(i) $s_{3}(r) \leq \mathscr{I} s_{2}(r) \leq \mathscr{I} s_{1}(r)$.

(ii) $s_{3}(r)=s_{2}(r)=s_{1}(r)$ se e somente se o segmento de $\gamma$ ligando $r$ a $s_{1}(r)$ for acronal.

Prova. (i) Imediato, assim como (ii) $\Rightarrow$. Resta apenas provar (ii) $\Leftarrow$. A saber, suponha que $s_{3}(r)$ coincide com $s_{2}(r)$. Então, o segmento geodésico nulo ligando $q$ a $s_{2}(r)$ deve pertencer a $\gamma$, pois caso contrário haveria um segmento geodésico quebrado ligando $r$ a $s_{3}(r)$, contradizendo a definição do último (isto, em particular, prova que $s_{1}(r)=s_{3}(r)$ mesmo se nós só assumirmos que $s_{2}(r)=$ $\left.s_{3}(r)\right)$. Se $\gamma$ não for acronal, uma vez mais temos uma contradição com a definição de $s_{3}(r)$. 
OBSERVAÇÃo 1.8 Resultados análogos às Proposições 1.11 e 1.12 valem se trocarmos o futuro com o passado.

Agora, seja de agora em diante $\mathscr{O}_{p, q} \subset \mathscr{M}$ um diamante relativamente compacto possuindo uma superfície de CAUCHY contrátil - qualquer diamante suficientemente pequeno satisfaz ambas as condições. Consideremos agora a região

$$
\mathscr{Q}_{p, q}=\bigcap_{\substack{r \in \partial I^{-}(p, \overline{\mathscr{M}}) \cap \mathscr{F}, s \in \partial I^{+}(q, \overline{\mathscr{M}}) \cap T(r)}} \mathscr{W}_{r, s} .
$$

Segue naturalmente desta definição que $\mathscr{Q}_{p, q} \supset \mathscr{O}_{p, q}$, é causalmente completa, por ser a intersecção de regiões causalmente completas, e

$$
\mathscr{Q}_{p, q} \cap J^{+}(q, \overline{\mathscr{M}})=\mathscr{Q}_{p, q} \cap J^{-}(p, \overline{\mathscr{M}})=\varnothing .
$$

Em AdS, $\mathscr{Q}_{p, q}=\mathscr{O}_{p, q}$. Para espaços-tempos AAdS assintoticamente simples satisfazendo as hipóteses do Teorema 1.3, no entanto, pode acontecer que $\mathscr{Q}_{p, q} \supsetneqq \mathscr{O}_{p, q}$. Analogamente, definindo $\mathscr{E}_{p, q} \doteq \partial I^{+}(p, \mathscr{M}) \cap \partial I^{-}(q, \mathscr{M})$, vamos começar a partir de

$$
\widetilde{\mathscr{Q}}_{p, q}=\bigcap_{r \in \mathscr{E}_{p, q}} \mathscr{W}_{s_{3}^{\prime}(r), s_{3}(r)},
$$

onde $s_{3}^{\prime}(r)$ corresponde a $s_{3}(r)$ se trocarmos o futuro com o passado no enunciado da Proposição 1.12. Aqui, $\widetilde{\mathscr{Q}}_{p, q} \subset \mathscr{Q}_{p, q}$ é novamente causalmente completo, se não-vazio. Entretanto, se a métrica bem "fundo" no interior é suficientemente "distorcida", fazendo com que um número suficiente de geradores nulos de, digamos, $\partial I^{-}(q, \mathscr{M})$ adquira pares de pontos conjugados entre $\mathscr{E}_{p, q}$ e $\mathscr{I}$, até onde sabemos (Proposição 1.12, página 25) $\widetilde{\mathscr{Q}}_{p, q}$ pode muito bem ser vazio. Isto é sugerido pelas seguintes observações:

1. Num espaço-tempo causalmente simples, qualquer diamante relativamente compacto $\mathscr{O}_{p, q}$ é uma região globalmente hiperbólica, para a qual qualquer superfície de CAuCHy possui fronteira igual a $\mathscr{E}_{p, q}$;

2. Qualquer região causalmente completa $\mathscr{U}$ possui a seguinte propriedade: se $\mathscr{S} \subset$ $\mathscr{U}$ é um conjunto fechado e acronal com respeito a $\mathscr{U}$, então $D(\mathscr{S}) \subset \mathscr{U}$.

Ambas as observações juntas mostram que, se $r \in \mathscr{E}_{p, q}$ é tal que um gerador nulo de, digamos, $\partial I^{-}(q, \mathscr{M})$ cruzando $r$ adquire um par de pontos conjugados entre $r$ e $s_{1}(r)$, então, por simplicidade causal, segue que existe uma vizinhança de $q$ causalmente disjunta de $s_{3}(r)$, e, portanto, $I^{-}\left(s_{3}(r), \overline{\mathscr{M}}\right)$ não pode conter uma superfície de CAUCHY para $\mathscr{O}_{p, q}$. Uma vez que, por outro lado, isto não exclui tampouco a possibilidade de que $\widetilde{\mathscr{Q}}_{p, q}$ possa conter pontos fora de $\mathscr{O}_{p, q}$, não é nem um pouco claro se as coleções de 
$\mathscr{Q}_{p, q}$ 's e $\widetilde{\mathscr{Q}}_{p, q}$ 's produzem bases para a topologia de $\mathscr{M}$ ou não.

Uma maneira de contornar estes problemas poderia ser restringir nossas considerações a diamantes suficientemente pequenos, tais que nenhum dos geradores nulos de $\partial I^{+}(q, \overline{\mathscr{M}})$ pode percorrer um caminho longo o suficiente além de $q$ que o permita adquirir um par de pontos conjugados. Há, no entanto, uma situação na qual não importa quão pequena a extensão, esta sempre deixará de ser acronal: é quando o próprio $q$ é conjugado a $s_{1}(r)$. Neste caso limite, $s_{1}(r)=s_{2}(r)$ mas $s_{2}(r) \neq s_{3}(r)$.

Mostraremos agora que a chave para o fim destes problemas é tentar construir uma região similar a $\widetilde{\mathscr{Q}}_{p, q}$, mas empregando, ao invés dos pontos $s_{3}(r), s_{3}^{\prime}(r)$ para $r \in \mathscr{E}_{p, q}$, os pontos para os quais o problema acima, implicado pela Proposição 1.12, é, num certo sentido, "minimizado". Para levarmos a cabo tal tarefa e assim otimizarmos a nossa construção, começaremos de um ponto de vista diferente, que acabará por mostrar que a situação crítica mencionada no parágrafo anterior é excluída por completeza geodésica nula.

Primeiro, notar que, por meio de um argumento similar ao empregado em [HE73] e [Wal84] para provar a existência de uma estrutura de variedade topológica (LIPSCHITz) para fronteiras acronais, pode-se mostrar que $\mathscr{E}_{p, q}$ é localmente o gráfico de uma função localmente LIPSCHITZ a valores em $\mathbb{R}$ com $d-2$ argumentos, e portanto é uma subvariedade topológica (LIPSCHITZ) acausal, compacta e mergulhada em $\mathscr{M}$, com codimensão 2. Perceber também que pode-se parametrizar de maneira $\mathscr{C}^{\infty}$ a família de geradores de tipo tempo de $\left(\mathscr{I}, \bar{g}^{(0)}\right)$ por meio de uma superfície de CAUCHY $\mathscr{S}$ deste último, homeomorfa a $S^{d-2}$ e portanto também compacta. Seja $t$ o parâmetro afim comum aos geradores de tipo tempo de $(\mathscr{I}, b)$ mencionados acima. Defina a função

$$
\tau: \mathscr{E}_{p, q} \times \mathscr{S} \ni(r, \theta) \mapsto \tau(r, \theta) \in \mathbb{R}
$$

onde

$$
\partial I^{+}(R, \overline{\mathscr{M}}) \cap T(\theta)=\{T(\theta)(\tau(r, \theta))\}
$$

A Proposição 1.11 mostra que a definição de $\tau$ não é vazia. Mais ainda:

PROPOSIÇÃo $1.13 \tau$ é contínua por cima em $r$ para $\theta$ fixo.

Prova. Seja $\epsilon>0$. $r$ fica no passado cronológico do ponto $T(\theta)(\tau(r, \theta)+\epsilon)$, e portanto existe uma vizinhança aberta $U$ de $r$ em $\mathscr{E}_{p, q}$ que fica no passado cronológico de $T(\theta)(\tau(r, \theta)+\epsilon)$. Portanto, para todo $r^{\prime} \in U$, devemos ter $\tau\left(r^{\prime}, \theta\right)<$ $\tau(r, \theta)+\epsilon$. 
Pode-se ainda provar que $\tau$ é localmente Lipschitziana em $\theta$ para $r$ fixo, mas isto não será usado no que se segue. A função $\tau(., \theta)$ será denominada potencial (futuro) de FERMAT com respeito a $\theta .^{8}$ O nome é remanescente do princípio de HuYgensFERMAT em óptica geométrica (ver, por exemplo, páginas 249-250 de [Arn89] para uma bela demonstração). Agora, estenda a definição de $\tau(., \theta)$ para o fecho $\overline{\mathscr{F} p, q}$ de alguma superfície de CAUCHY $\mathscr{F}_{p, q}$ para $\mathscr{O}_{p, q}$, denotando-a pelo mesmo símbolo, uma vez que não há aqui margem para ambigüidade. Pelo mesmo argumento empregado na Proposição 1.13 , segue que $\tau(., \theta)$ é contínua por cima em $\overline{\mathscr{F}} p, q$. Uma vez que tanto $\mathscr{E}_{p, q}=\partial \mathscr{F}_{p, q}$ como $\overline{\mathscr{F}_{p, q}}$ são subconjuntos fechados do compacto $\overline{\mathscr{O}_{p, q}}$, eles mesmos são compactos. Segue então de um resultado padrão em Análise (ver, por exemplo, páginas 110-111 de [KF75]) que $\tau(., \theta)$ possui um máximo tanto em $\overline{\mathscr{F}}_{p, q}$ como em $\mathscr{E}_{p, q}$. O próximo Teorema mostra que $\tau(., \theta)$ possui, de fato, uma propriedade típica de potenciais:

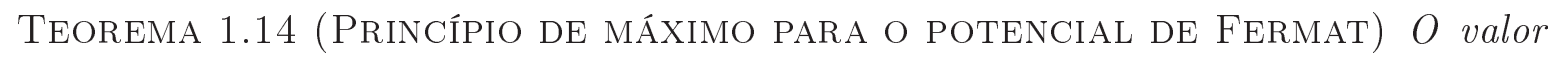
máximo de $\tau(., \theta)$ em $\overline{\mathscr{F}_{p, q}}$ é atingido em $\mathscr{E}_{p, q}$.

Prova. Seja $r$ um ponto de $\mathscr{E}_{p, q}$ onde $\tau(., \theta)$ atinge seu máximo em $\mathscr{E}_{p, q}$, e seja $r^{\prime}$ um ponto de $\mathscr{F}_{p, q}$ tal que $\tau\left(r^{\prime}, \theta\right) \geq \tau\left(r^{\prime}, \theta\right)$. Neste caso, é óbvio que $\mathscr{E}_{p, q}$ fica no passado causal de $T(\theta)\left(\tau\left(r^{\prime}, \theta\right)\right)$. Pegue um segmento de curva em $\frac{\mathscr{F}_{p, q}}{\mathscr{F}, q}$ começando em $r^{\prime}$, inicialmente apontando para fora de $J^{-}\left(T(\theta)\left(\tau\left(r^{\prime}, \theta\right)\right), \overline{\mathscr{M}}\right)$ e terminando em algum ponto de $\mathscr{E}_{p, q}$. Então, qualquer tal segmento de curva deve cruzar $\partial I^{-}\left(T(\theta)\left(\tau\left(r^{\prime}, \theta\right)\right), \overline{\mathscr{M}}\right)$ pelo menos uma vez mais depois de $r^{\prime}$, e antes de atingir (ou atingindo) $\mathscr{E}_{p, q}$. Isso mostra que $\partial I^{-}\left(T(\theta)\left(\tau\left(r^{\prime}, \theta\right)\right), \overline{\mathscr{M}}\right) \cap \overline{\mathscr{F}} p, q$ contém um subconjunto aberto $X$ de $\mathscr{F}_{p, q}$ fora do passado causal de $T(\theta)\left(\tau\left(r^{\prime}, \theta\right)\right)$.

O restante da prova é análogo à prova do teorema de singularidade de PEnRose [HE73, Wal84]: a saber, mostraremos que as propriedades de $\partial X$ implicam na existência de uma geodésica nula incompleta em $(\mathscr{M}, g)$. Primeiro, vamos mostrar que o conjunto fechado e acausal $\partial X=\partial I^{-}\left(T(\theta)\left(\tau\left(r^{\prime}, \theta\right)\right), \overline{\mathscr{M}}\right) \cap \overline{\mathscr{F}_{p, q}}$ é aprisionado no passado, i.e., $\partial I^{-}(X, \mathscr{M})$ é compacto. As geodésicas nulas passadas "entrando" em $\partial X$ constituem o horizonte de CAuchy passado de $X$, o qual é assim contido em $\overline{\mathscr{O}_{p, q}}$ e, portanto, compacto, por ser fechado. As geodésicas nulas "saindo" são precisamente os geradores nulos de $\partial I^{-}\left(T(\theta)\left(\tau\left(r^{\prime}, \theta\right)\right), \overline{\mathscr{M}}\right)$ que cruzam $\partial X$. Adotemos uma parametrização afim comum aos geradores nulos de $\partial I^{-}\left(T(\theta)\left(\tau\left(r^{\prime}, \theta\right)\right), \overline{\mathscr{M}}\right)$ tal que o zero do parâmetro afim corresponde a $\partial X$. Então, seja $t_{0}$ o maior valor do parâmetro afim para o qual um extremo passado de $\partial I^{-}\left(T(\theta)\left(\tau\left(r^{\prime}, \theta\right)\right), \overline{\mathscr{M}}\right)$ é atingido. Este valor deve ser finito, pois toda geodésica nula inextensível deve adquirir um par de pontos conjugados antes de atingir o infinito, embora o valor do parâmetro afim num extremo passado do segmento de gerador nulo começando, digamos, em $r^{\prime \prime} \in \partial X$ pode ser zero se

${ }^{8} \mathrm{Na}$ verdade, $\tau$ também depende da escolha de folheação de $\mathscr{I}$ por superfícies de CAUCHY, mas tal dependência é irrelevante para o uso que faremos de $\tau$ e será ignorada. 
$r^{\prime}$ acontece de ser ele mesmo um extremo passado. De qualquer forma, a porção de $\partial I^{-}\left(T(\theta)\left(\tau\left(r^{\prime}, \theta\right)\right), \overline{\mathscr{M}}\right)$ no passado causal de $\partial X$, sendo fechada, possui uma imagem inversa fechada no compacto $\left[0, t_{0}\right] \times \partial X$ pela parametrização escolhida para os geradores nulos, e assim sendo é compacta. Logo, o conjunto $\partial I^{-}(\partial X, \mathscr{M})=H^{-}(X) \cup \partial X \cup\left(\partial I^{-}\left(T(\theta)\left(\tau\left(r^{\prime}, \theta\right)\right), \overline{\mathscr{M}}\right) \cap J^{-}(\partial X, \mathscr{M})\right)$ é um subconjunto compacto e acronal de $\mathscr{M}$, como afirmamos.

Entretanto, qualquer espaço-tempo causalmente simples é estavelmente causal [BEE96]. Ou seja, pode-se folhear de maneira $\mathscr{C}^{\infty} \mathscr{M}$ por superfícies "de tempo constante" de tipo espaço e codimensão 1. Pela estrutura do infinito conforme, estas superfícies não podem ser compactas. Mais ainda, cada órbita de tipo tempo desta folheação cruza um conjunto acronal no máximo uma vez. Seguindo estas órbitas, pode-se mapear continuamente $\partial I^{-}(\partial X, \mathscr{M})$ numa folha de tipo espaço desta folheação. Como a imagem desta aplicação é compacta, ela deve ter uma fronteira não-vazia. É, no entanto, sabido que um conjunto da forma $\partial I^{-}(Y, \mathscr{M}), Y \subset \mathscr{M}$ é uma subvariedade topológica sem fronteira de $\mathscr{M}$. Isto mostra que algum gerador nulo de $\partial I^{-}\left(T(\theta)\left(\tau\left(r^{\prime}, \theta\right)\right), \overline{\mathscr{M}}\right)$ deve atingir uma singularidade antes de atingir seu extremo passado. Mas isto entra em conflito com a completeza geodésica nula de $\widehat{\mathscr{M}}$, implicada por simplicidade assintótica. Portanto, nenhum ponto de $\mathscr{F}_{p, q}$ pode atingir um máximo para $\tau(., \theta)$ em $\overline{\mathscr{F}_{p, q}}$ - este sempre ocorre em $\mathscr{E}_{p, q}$.

A Proposição 1.13 e o Teorema 1.14 juntos mostram que, para cada $\theta$, sempre há $\underline{\text { um } r} \in \mathscr{E}_{p, q}$ tal que, dada qualquer superfície de CAUCHY $\mathscr{F}_{p, q}$ para $\mathscr{O}_{p, q}$, o conjunto $\overline{\mathscr{F}_{p, q}}$ sempre fica no passado causal de $T(\theta)(\tau(r, \theta))$. Pela Proposição 1.12 e pelas observações acima, isso só pode ocorrer se o segmento geodésico nulo acronal $\gamma(r, \theta)$ ligando $r$ a $T(\theta)(\tau(r, \theta))$ cruza $q$. Logo, este ponto de máximo é único: suponha o contrário. Então, haveria outro $r^{\prime} \in \mathscr{E}_{p, q}$ tal que existe um segmento geodésico nulo acronal $\gamma\left(r^{\prime}, \theta\right)$ ligando $r^{\prime}$ a $T(\theta)\left(\tau\left(r^{\prime}, \theta\right)\right)=T(\theta)(\tau(r, \theta))$ e cruzando $q$. Agora, considere o segmento de curva $\gamma^{\prime}(r, \theta)$, que coincide $\operatorname{com} \gamma(r, \theta)$ de $r$ a $q$, e coincide com $\gamma\left(r^{\prime}, \theta\right)$ de $q$ a $T(\theta)(\tau(r, \theta))$. Este segmento é necessariamente quebrado, o que conflita com a acronalidade de $\gamma(r, \theta)$. Trocando os papéis de $r$ e $r^{\prime}$, pode-se ver que este argumento também entra em conflito com a acronalidade de $\gamma\left(r^{\prime}, \theta\right)$.

Percebamos, contudo, que um $r \in \mathscr{E}_{p, q}$ arbitrário não precisa maximizar $\tau(., \theta)$ para algum $\theta$. As duas situações em que isto de fato não pode ocorrer são:

1. $r$ é conjugado a $q$ ao longo de um gerador nulo de $\partial I^{-}(q, \mathscr{M})$ - qualquer extensão futura desse gerador além de $q$ não será acronal;

2. $q$ é conjugado a $s_{2}(r)$ ao longo de um gerador nulo de $\partial I^{+}(q, \overline{\mathscr{M}})$, pelas observações feitas acima. 
A segunda instância, no entanto, é excluída pela nossa linha de raciocínio, pois torna impossível, pela Proposição 1.12 e pelo Teorema 1.14 que $\tau(., \theta)$ atinja um máximo em $\mathscr{E}_{p, q}$. Isto não pode ocorrer, uma vez que para cada $\theta$ um máximo precisa existir pela Proposição 1.13. A primeira instância pode ser contornada se pegarmos $\mathscr{O}_{p, q}$ contido, digamos, numa vizinhança normal convexa, o que sempre pode ser feito, pois aqui $(\mathscr{M}, g)$ é fortemente causal. Pode-se ir além e tomar $\mathscr{O}_{p, q}$ suficientemente pequeno (conquanto não-vazio) de modo que todo $r \in \mathscr{E}_{p, q}$ é um máximo de $\tau(., \theta)$ para algum $\theta$, pois o único obstáculo a isto seria a segunda instância acima, excluída pelo argumento aqui apresentado. Todos estes resultados possuem uma contraparte para o passado, se trocarmos $q$ com $p$ e invertermos a orientação temporal.

Em suma, mostramos que qualquer $\mathscr{O}_{p, q}$ suficientemente pequeno pode sempre ser precisamente envolvido por cunhas. Neste caso, qualquer ponto que não pertença a $\overline{\mathscr{O}_{p, q}}$ cai ou no futuro cronológico de $\partial I^{-}(q, \mathscr{M})$ ou no passado cronológico de $\partial I^{+}(p, \mathscr{M})$, e, como tal, falhará em pertencer a qualquer cunha envolvendo $\mathscr{O}_{p, q}$. Já que os pontos em $\partial \mathscr{O}_{p, q}$ estão automaticamente excluídos da intersecção por construção, conclui-se que $\mathscr{O}_{p, q}=\mathscr{Q}_{p, q}$ para $\mathscr{O}_{p, q}$ suficientemente pequeno. Mais ainda, nesta situação, cada cunha na definição (1.35) de $\mathscr{Q}_{p, q}$ está garantidamente contida em algum domínio de PoincARÉ. Note ainda que o procedimento que propusemos permite também envelopar os diamantes regulares (suficientemente pequenos) definidos em [GLRV01]. 


\section{CAPÍTULO 2}

\section{Dinâmica e evolução temporal}

Because I know that time is always time And place is always and only place

And what is actual is actual only for one time

And only for one place ix

T. S. ELIOT

"I. Because I do not hope to turn again"

(Ash-Wednesday $\left.{ }^{\mathrm{x}}, 1930\right)$

Fez-se uso extensivo da estrutura global dos espaço-tempos AAdS no Capítulo 1, mas as equações de EINSTEIN exerceram um papel secundário nestes desenvolvimentos. Todavia, ficou claro ao longo do Capítulo anterior que geometrias não-triviais no interior de um espaço-tempo AAdS provocam uma distorção da estrutura causal em longas distâncias, e que, portanto, deve ser perceptível do ponto de vista de uma teoria de observáveis locais na fronteira. Assim sendo, é altamente desejável estabelecer uma conexão mais quantitativa entre esses efeitos e a própria dinâmica gravitacional, visto que a conjectura de MALDACENA e muitas de suas conseqüências envolvem efeitos gravitacionais de maneira essencial.

\subsection{Abordagem variacional para a gravitação clássica. Fundamentos conceituais}

As equações de Einstein (1.1) são obtidas na abordagem Lagrangiana por meio do princípio variacional de Einstein-Hilbert: para todo compacto $K \Subset \mathscr{M}$ com fronteira $\mathscr{C}^{\infty}$ por partes (assumimos que o conjunto dos pontos não-diferenciáveis de 
$\partial K$ tem medida de Lebesgue $(d-1)$-dimensional zero), o funcional de ação

$$
S_{K}[g] \doteq \frac{1}{16 \pi G_{d}} \int_{K} \underbrace{(R(g)+2 \Lambda)}_{\doteq \mathscr{L}_{\Lambda}(g)} \sqrt{|g|} d x^{(1)}
$$

é estacionário para variações de $g$ arbitrárias ao redor de uma solução do princípio variacional, suportadas no interior de $K$. A saber, uma variação finita de $g$ é obtida a partir de uma curva $\mathscr{C}^{\infty}$ de métricas $g_{\lambda}, \lambda \in(-1,1)$, que diferem de $g$ num compacto $K_{1} \subset \operatorname{int}(K)$ e tais que $g_{0}=g$. A variação infinitesimal (vetor tangente) correspondente ao redor de $g$ é dada por $\delta g=\left.\frac{d}{d \lambda}\right|_{\lambda=0} g_{\lambda}$. A variação correspondente em (2.1) é dada por

$\left(\delta S_{K}\right)[g, \delta g]=\int_{K}[\nabla^{a} \underbrace{\left(\nabla^{b} \delta g_{a b}-g^{c d} \nabla_{a} \delta g_{c d}\right)}_{\dot{\equiv} \theta_{a}(g, \delta g) ;}+\left(\operatorname{Ric}_{a b}-\frac{1}{2} R g_{a b}+\Lambda g_{a b}\right) \delta g^{a b}] \sqrt{|g|} d x$,

onde o primeiro termo do segundo membro é a divergência de uma 1-forma, dual de HoDGE da $(d-1)$-forma $\theta^{a_{1}} \sqrt{|\operatorname{det} g|}\left[_{\left[a_{1} \cdots a_{d}\right]}\right.$, que se anula em $\partial K$ para a classe de variações considerada. O segundo termo é o tensor de Einstein $G(g)_{a b} \doteq \operatorname{Ric}(g)_{a b}-$ $\frac{1}{2} R(g) g_{a b}$, mais a parte da constante cosmológica $\Lambda$. Assim, temos as equações de EinsTEIN (1.1). Notemos que o uso de um domínio de integração $K$ compacto reflete o fato de que o princípio variacional que leva a 1.1 é inerentemente local, como toda teoria de campos.

Finalizamos esta Seção ressaltando aqui que, para qualquer vetor $T^{a}$, a expressão $\left(\operatorname{Ric}(g)_{a b}-\frac{1}{2} R(g) g_{a b}+\Lambda g_{a b}\right) T^{b}$ não possui derivadas de segunda ordem na direção $T^{a}$. Se $T^{a}$ é tipo tempo, determinando assim (localmente) um fluxo temporal, vemos que as componentes $\left(\operatorname{Ric}(g)_{a b}-\frac{1}{2} R(g) g_{a b}+\Lambda g_{a b}\right) T^{b}=0$ das equações de Einstein são, na verdade, vínculos, expressando a simetria destas por transformações gerais de coordenadas (ponto de vista passivo) ou, equivalentemente em termos matemáticos, por difeomorfismos locais (ponto de vista ativo). O significado físico deste fato, segundo Einstein, é que a gravitação não possui uma noção intrínseca de evolução temporal (dinâmica), pois a própria escolha de coordenada temporal é uma simetria - tal escolha, portanto, deve ser feita por meio de um procedimento físico concreto, de maneira tal que as leis físicas que regem tais procedimentos no nosso fundo gravitacional sejam independentes dessa escolha. Há duas maneiras de fazer isso: uma local - pela especificação de um tensor de energia-momento não-trivial - e outra global, caso a métrica

\footnotetext{
${ }^{1} G_{d}$ é a constante de Newton $d$-dimensional; daqui em diante, escolhemos unidades $G_{d}=(16 \pi)^{-1}$, de modo que o fator numérico multiplicando o segundo membro de (2.1) passe a ser igual a 1.
} 
se aproxime de alguma geometria de fundo fixa em distâncias suficientemente longas, para a qual tal escolha pode ser feita naturalmente. Esta última circunstância inclui precisamente o caso de espaços-tempos AAdS.

Na próxima Seção, faremos uso deste fato para explorar em detalhe a geometria de espaços-tempos (localmente) AAdS nas proximidades do infinito conforme.

\subsection{A expansão de FEFFERMAN-GRAHAM}

Consideremos o caso de espaços-tempos tipo AdS $(\mathscr{M}, g)$ como na Definição 1.4, da qual adotamos a notação. Vamos agora escrutinar a forma das soluções de (1.1) para tais espaços-tempos numa vizinhança de colarinho $\overline{\mathscr{U}}_{\epsilon} \cong \mathscr{I} \times[0, \epsilon) \ni(x, z), \epsilon>0$, de $\mathscr{I}$ em $\widetilde{\mathscr{M}}$. Isto será feito por meio da expansão assintótica obtida por FEFFERMAN e Graham [FG85], cuja dedução apresentamos abaixo. No que se segue, seguimos de perto [HIM05] e, em menor medida, [Gra00, GL91, HSS01].

Uma maneira equivalente de escrever (1.1) é diretamente em termos do tensor de RiCCI, resultando em

$$
\operatorname{Ric}(g)_{a b}=\frac{2 \Lambda}{d-2} g_{a b} .
$$

Invocando agora a fórmula (A.25) da transformação conforme de $\operatorname{Ric}(g)$ em $\operatorname{Ric}(\bar{g})$, $\bar{g}=z^{2} g$

$$
\operatorname{Ric}(g)_{a b}=\operatorname{Ric}(\bar{g})_{a b}+\frac{d-2}{z} \bar{\nabla}_{a} \bar{\nabla}_{b} z+\bar{g}_{a b} \bar{g}^{c d}\left(\frac{1}{z} \bar{\nabla}_{c} \bar{\nabla}_{d} z-\frac{d-1}{z^{2}} \bar{\nabla}_{c} z \bar{\nabla}_{d} z\right),
$$

temos a expressão de(2.3) em termos de $\bar{g}$

$$
\operatorname{Ric}(\bar{g})_{a b}+\frac{d-2}{z} \bar{\nabla}_{a} \bar{\nabla}_{b} z+\bar{g}_{a b} \bar{g}^{c d}\left(\frac{1}{z} \bar{\nabla}_{c} \bar{\nabla}_{d} z-\frac{d-1}{z^{2}} \bar{\nabla}_{c} z \bar{\nabla}_{d} z\right)=\frac{2 \Lambda}{(d-2) z^{2}} \bar{g}_{a b} .
$$

Note, em particular, que se multiplicarmos (2.4) por $z^{2}$ e tomarmos $z=0$, segue que $d z$ necessariamente satisfaz

$$
\bar{g}^{-1}(d z, d z) \Gamma_{\mathscr{I}}=-\frac{2 \Lambda}{(d-1)(d-2)}=\frac{1}{R^{2}},
$$

onde $R$ é o raio AdS de $(\mathscr{M}, g)$, definido no Capítulo 1.

Restrinjamos agora (2.4) às hipersuperfícies tipo tempo $\mathscr{I}_{\epsilon}^{\prime} \doteq z^{-1}\left(\epsilon^{\prime}\right), 0 \leq \epsilon^{\prime}<\epsilon$. Primeiramente, vamos usar a liberdade que temos na escolha de $z$ para definir um sistema de coordenadas Gaussianas normais em $\mathscr{U}_{\epsilon}$ : 
LEMA 2.1 Dada qualquer métrica tipo AdS g satisfazendo as equações de EINSTEIN (2.3) em $\mathscr{U}_{\epsilon}$ para algum $\epsilon>0$, podemos escolher $z$ e $\epsilon$ de modo que ${ }^{(*)} \bar{g}^{-1}(d z, d z) \doteq \zeta^{2}$ é constante em $\mathscr{U}_{\epsilon}$. Em particular, $\zeta^{2}=R^{-2}$.

Prova. Sejam $z^{\prime}=e^{\sigma} z$ e $\bar{g}^{\prime}=e^{2 \sigma} \bar{g}$; então, $d z^{\prime}=e^{\sigma}(d z+z d \sigma)$, e daí

$$
\begin{aligned}
& \bar{g}^{\prime-1}\left(d z^{\prime}, d z^{\prime}\right)=\bar{g}^{-1}(d z+z d \sigma, d z+z d \sigma)= \\
= & \bar{g}^{-1}(d z, d z)+2 z \bar{g}^{-1}(d z, d \sigma)+z^{2} \bar{g}^{-1}(d \sigma, d \sigma) .
\end{aligned}
$$

Logo, a condição ${ }^{(*)}$ para $d z^{\prime}$ é equivalente à equação diferencial parcial não-linear de primeira ordem

$$
2 z \bar{g}^{-1}(d z, d \sigma)+z^{2} \bar{g}^{-1}(d \sigma, d \sigma)=\frac{\zeta^{2}-\bar{g}^{-1}(d z, d z)}{z},
$$

onde o segundo membro estende-se de maneira $\mathscr{C}^{\infty}$ a $z=0$ em razão de (2.5), o que por sua vez fixa $\zeta^{2}=R^{-2}$. Pelo método das características [Joh82] (ver também a Subseção 6.4 de [Hör90]), existe $\epsilon>0$ e uma única solução $\mathscr{C}^{\infty}$ desta equação em $\mathscr{U}_{2 \epsilon}$ para valores arbitrários de $\sigma\lceil\mathscr{I}$. O resultado segue globalmente empregando-se uma partição de unidade subordinada ao recobrimento $\left\{\mathscr{U}_{2 \epsilon}, \mathscr{M} \backslash\right.$ $\left.\overline{\mathscr{U}_{\epsilon}}\right\}$ de $\overline{\mathscr{M}}$.

Aplicando a escolha de $z$ ditada pelo Lema 2.1 a (2.4), vemos que o último termo do primeiro membro cancela o segundo membro, resultando em

$$
\operatorname{Ric}(\bar{g})_{a b}+\frac{d-2}{z} \bar{\nabla}_{a} \bar{\nabla}_{b} z+\bar{g}_{a b} \bar{g}^{c d} \frac{1}{z} \bar{\nabla}_{c} \bar{\nabla}_{d} z=0 .
$$

Note ainda que, pelas fórmulas (A.22-A.24) (páginas 115-117), a multiplicação de $\bar{g}$ por uma constante $K=\kappa^{2}>0$ não modifica a conexão de LEVI-CivitA, nem os tensores de RiEMANN e de RiCCi. Assim, podemos fazer a substituição $g \mapsto-\frac{(d-1)(d-2)}{2 \Lambda} g=$ $R^{2} g$ e, simultaneamente, tomar $\Lambda=-\frac{(d-1)(d-2)}{2}$, no que resulta que $\bar{g}^{-1}(d z, d z)=1$ em $\mathscr{U}_{\epsilon}$. Neste caso, denotando por $Z_{a} \doteq \bar{\nabla}_{a}^{2} z$ a normal (unitária!) à folheação de $\mathscr{U}_{\epsilon}$ induzida pela nossa escolha de $z$, segue que $K_{a b} \doteq-\bar{\nabla}_{a} Z_{b}$ é a curvatura extrínseca de $\mathscr{I}_{z}$. Podemos, assim, escrever $\bar{g}$ como

$$
\bar{g}(x, z)_{a b}=\bar{g}^{(0)}(x, z)_{a b}+Z_{a} Z_{b}
$$

onde $\bar{g}^{(0)}(z,$.$) é a métrica induzida em cada uma destas hipersuperfícies \left(\bar{g}^{(0)}(x, 0) \doteq\right.$ $\left.\bar{g}^{(0)}(x)\right)$. Notando que

$$
Z^{a} K_{a b}=-\left(\bar{\nabla}^{a} z\right) \bar{\nabla}_{a} \bar{\nabla}_{b} z=-\left(\bar{\nabla}^{a} z\right) \bar{\nabla}_{b} \bar{\nabla}_{a} z=-\frac{1}{2} \bar{\nabla}_{b}\left(\bar{\nabla}^{a} z \bar{\nabla}_{a} z\right)=0
$$

podemos projetar (2.6) na sua parte tangente às $\mathscr{I}_{z}$ 's: 


$$
\bar{g}^{(0)}(z)_{a}^{c} \bar{g}^{(0)}(z)_{b}^{d} \operatorname{Ric}(\bar{g})_{c d}-\frac{d-2}{z} K_{a b}-\frac{1}{z} \bar{g}^{(0)}(z)_{a b} \operatorname{Tr} K=0 .
$$

onde $\operatorname{Tr} K \doteq \bar{g}^{(0)}(z)^{c d} K_{c d}$ é a curvatura média. Finalmente, aplicando a equação de Gauss (A.3) a $\operatorname{Ric}(\bar{g})$

$$
\bar{g}^{(0)}(z)_{a}^{c} \bar{g}^{(0)}(z)_{b}^{d} \operatorname{Ric}(\bar{g})_{c d}=\operatorname{Riem}(\bar{g})_{a c b d} Z^{c} Z^{d}+\operatorname{Ric}\left(\bar{g}^{(0)}\right)_{a b}+K_{a}^{e} K_{e b}-(\operatorname{Tr} K) K_{a b},
$$

e fazendo uso da identidade (análoga à equação de RicCATi (A.6))

$$
\operatorname{Riem}(\bar{g})_{a c b d} Z^{c} Z^{d}=Z^{e} \bar{\nabla}_{e} K_{a b}-K_{a}^{e} K_{e b},
$$

chegamos, após multiplicação por z nos dois membros, em

$$
z \underbrace{Z^{e} \bar{\nabla}_{e} K_{a b}}_{=-\frac{1}{2} \partial_{z}^{2} \bar{g}^{(0)}(z)}+z \operatorname{Ric}\left(\bar{g}^{(0)}\right)_{a b}-z(\operatorname{Tr} K) K_{a b}-(d-2) K_{a b}-\bar{g}^{(0)}(z)_{a b} \operatorname{Tr} K=0,
$$

que é a equação de evolução que procurávamos. ${ }^{2}$ Note, contudo, que (2.10) possui vínculos, pois a contração das equações de EINSTEIN (2.3) com qualquer vetor $X^{a}$ não possui derivadas de segunda ordem na direção de $X^{a}$. A contração de (2.6) e (2.8) com $\bar{g}^{(0) a b}$, mais a equação de CODAZZI-MAINARDi (A.4) juntamente com (2.6), resultam respectivamente nas equações de vínculo (2.11) e (2.12) para a evolução de $\left(\bar{g}^{(0)}, K\right)$ ditada por (2.10):

$$
\begin{aligned}
R\left(\bar{g}^{(0)}\right)+K_{a b} K^{a b}-(\operatorname{Tr} K)^{2}+2 \frac{d-2}{z} \operatorname{Tr} K & =0 \\
\bar{\nabla}_{a}^{(0)} K_{b}^{a}-\bar{\nabla}_{b}^{(0)} \operatorname{Tr} K & =0 .
\end{aligned}
$$

A conservação dos vínculos (2.11-2.12) ao longo da evolução é garantida pelas identidades de BiAnCHI contraídas $\nabla^{a} G_{\Lambda}(g)_{a b}=0$, reexpressas em termos de $\bar{g}$.

(2.10) constitui um sistema Fuchsiano não-linear de segunda ordem (ver, por exemplo, [Kic04] para uma definição do conceito de sistema Fuchsiano no caso não-linear), que pode ser formalmente resolvido adotando-se inicialmente o ansatz de séries de TAYLOR

$$
\bar{g}^{(0)}(z)=\bar{g}^{(0)}+\sum_{j=1}^{\infty} z^{j} \bar{g}^{(j)}
$$

\footnotetext{
${ }^{2}$ Esta equação é ligeiramente diferente da equação de evolução derivada em [FG85] e estudada em [Gra00, GL91, HSS01]. Na verdade, FEFFERMAn e GRAham partem de considerações diferentes das nossas em [FG85] para chegar em seu análogo de (2.10).
} 
Note que, tomando-se $z=0$ em (2.10), temos que $K_{a b}\lceil\mathscr{I}=0$, fixando assim nossos dados iniciais $\left(g^{(0)}, 0\right)$. Os coeficientes $g^{(j)}$ são obtidos aplicando-se $\partial_{z}^{j-1}$ nos dois membros de $(2.10)$, resultando em

$$
\begin{gathered}
\frac{d-1-j}{2} \partial_{z}^{j} \bar{g}^{(0)}(z)_{a b}+\frac{1}{2} \bar{g}^{(0)}(z)_{a b} \bar{g}^{(0)}(z)^{c d} \partial_{z}^{j} \bar{g}^{(0)}(z)_{c d}= \\
=\left(\text { só termos com } \partial_{z}^{k} \bar{g}^{(0)}(z), k<j\right) .
\end{gathered}
$$

Para uso futuro, é conveniente separarmos (2.12-2.14) nas partes de traço $\operatorname{Tr} K$ e de traço nulo $P_{a b} \doteq K_{a b}-\frac{1}{d-1} \bar{g}^{(0)}(z)_{a b} \operatorname{Tr} K$

$$
\begin{aligned}
(d-2-j) P_{b}^{(j) a}= & \operatorname{Ric}\left(\bar{g}^{(0)}\right)_{b}^{(j-1) a}-\frac{1}{d-1} R\left(\bar{g}^{(0)}\right)^{(j-1)} \delta_{b}^{a}+ \\
& -\sum_{m=0}^{j-1}(\operatorname{Tr} K)^{(m)} P_{b}^{(j-1-m) a} \\
(2 d-3-j)(\operatorname{Tr} K)^{(j)}= & R\left(\bar{g}^{(0)}\right)^{(j-1)}-\sum_{m=0}^{j-1}(\operatorname{Tr} K)^{(m)}(\operatorname{Tr} K)^{(j-1-m)},
\end{aligned}
$$

de onde podemos obter, invocando a identidade $\partial_{z} \bar{g}^{(0)}(z)_{a b}=-2 \partial_{z} \bar{g}^{(0)}(z)_{b c} K_{a}^{c}$, a forma explícita de (2.13)

$$
j \bar{g}^{(j)}=-2 \sum_{m=0}^{j-1}\left(\bar{g}_{b c}^{(m)} P_{a}^{(j-1-m) c}+\frac{1}{d-1} \bar{g}_{a b}^{(m)} K^{(j-1-m)}\right) .
$$

O vínculo "Hamiltoniano" (2.11) garante a invariância da solução de (2.10), a menos de difeomorfismos, por reparametrizações da variável $z$, e não exerce nenhum papel direto na determinação dos coeficientes da expansão (2.13).

Vemos, assim, que $\bar{g}^{(j)}, j<d-1$, é unicamente determinado por $\bar{g}^{(0)}$ em $\mathscr{I}$ e suas derivadas tangenciais a $\mathscr{I}$ (em particular, se $\bar{g}^{(0)}$ é a métrica do ESU, estes coeficientes devem coincidir com os coeficientes da métrica AdS numa carta similar numa vizinhança de $\mathscr{I})$. Em particular, como (2.10) é invariante pela transformação de paridade $z \mapsto-z$, segue que $\bar{g}^{(j)}=0$ para $j$ ímpar, $j<d-1$. Para $j=d-1$, as relações de recursão dadas por (2.14) são truncadas, e, neste caso, temos dois cenários possíveis:

(i) $d$ PAR: Uma vez mais, invariância por paridade exige $\operatorname{Tr} \bar{g}^{(d-1)}=0$, mas a parte sem traço de $\bar{g}^{(d-1)}$ é sujeita somente à lei de conservação $\bar{\nabla}^{(0) a} \bar{g}_{a b}^{(d-1)}=0$, proveniente de $(2.12)$.

(ii) $d$ ÍMPAR: (2.14) fixa não-trivialmente o traço de $\bar{g}^{(d-1)}$, e $(2.12)$ resulta em $\bar{\nabla}^{(0) a} \bar{g}_{a b}^{(d-1)}=$ $\bar{\nabla}_{b}^{(0)} \operatorname{Tr} \bar{g}^{(d-1)}$, prescrevendo assim a divergência da parte de traço nulo. 
No caso de $d$ impar, é necessário seguir a estratégia usual de solução de sistemas Fuchsianos, modificando o ansatz adotado inicialmente de modo a incluir termos da forma $z^{k} \log z, k \geq d-1$ (tais termos inexistem para $d$ par). Tal análise foi esboçada originalmente por FEFFERMAN e GRAHAM [FG85] para $\bar{g}^{(0)}$ com assinatura arbitrária, tendo sido analisada no caso de assinatura Euclidiana por vários autores, dentre os quais citamos em particular os trabalhos de GRAHAM e LEE [GL91] e GRAHAm [Gra00]. De grande importância foi o cálculo dos coeficientes $\bar{g}^{(j)}, j<d-1$, e do coeficiente $\bar{h}^{(d-1)}$ do termo proporcional a $z^{d-1} \log z$, bastante trabalhoso e realizado em detalhe para certos valores de $d$ por DE HARO, Skenderis e Solodukhin [HSS01]. Assim procedendo, a expansão de $\bar{g}$ ao redor de $z=0$, devidamente corrigida pelas considerações acima, fica

(i) $d$ PAR: $\bar{g}(z, x) \quad \sim_{z \backslash 0} \quad d z^{2}+\bar{g}^{(0)}(x)+\left(z^{2}\right) \bar{g}^{(2)}(x)+\cdots+\left(z^{2}\right)^{\left(\frac{d-2}{2}\right)} \bar{g}^{(d-2)}(x)+$

(ii) $d$ ÍMPAR: $\quad \bar{g}(z, x) \quad \sim_{z \backslash 0} \quad d z^{2}+\bar{g}^{(0)}(x)+\left(z^{2}\right) \bar{g}^{(2)}(x)+\cdots+\left(z^{2}\right)^{\left(\frac{d-3}{2}\right)} \bar{g}^{(d-3)}(x)+$

$$
+\left(z^{d-1} \log z\right) \bar{h}^{(d-1)}+z^{d-1} \bar{g}^{(d-1)}+\cdots \text {. }
$$

(entendemos (2.18) e (2.19), por ora, num caráter apenas assintótico - a questão de convergência será discutida mais adiante). O coeficiente $\bar{h}^{(d-1)}$, denominado tensor de FEFFERMAN-GRAHAM, possui propriedades notáveis: assim com $\bar{g}^{(j)} \operatorname{com} j<d-1$, só depende de $\bar{g}^{(0)}$ e suas derivadas tangenciais a $\mathscr{I}$; é conformalmente invariante; e se anula se e somente se a classe conforme de $g^{(0)}$ possui um representante que resolva as equações de Einstein sem matéria e com constante cosmológica nula (como, por exemplo, $\left.E S U_{d-1}\right)$. Neste último caso, não há coeficientes da forma $z^{j} \log z$ em (2.19), mas isso claramente deixa de ser verdade para perturbações de $g^{(0)}$ com suporte compacto e não provenientes de rescalonamento conforme local, o que afeta o cálculo de tensores de energia-momento para teorias de campo na fronteira obtidas pela correspondência AdS/CFT, causando uma anomalia no traço deste tensor e, assim, quebrando a invariância de escala. Tal anomalia, neste contexto, é denominada anomalia holográfica de WEYL [HS98, HS00].

Vamos aqui, ao invés de detalhar estes cálculos (para os quais não teremos uso no restante do presente trabalho, e que podem ser encontrados nas referências supracitadas), explorar mais detalhadamente as componentes restantes de $\operatorname{Riem}(\bar{g})$, de modo a precisar melhor a parte de $\bar{g}^{(d-1)}$ não determinada pelas relações de recursão (2.14). Primeiro, vamos simplificar (2.6) em termos de $K_{a b}$. Para tal, introduzimos o tensor de Schouten associado a $\bar{g}$

$$
S(\bar{g})_{a b} \doteq \frac{1}{d-2}\left(\operatorname{Ric}(\bar{g})_{a b}-\frac{1}{2(d-1)} R(\bar{g}) \bar{g}_{a b}\right) .
$$

Este tensor possui uma transformação conforme e uma relação envolvendo $\operatorname{Riem}(\bar{g})$ e 
$C(\bar{g})$ mais simples que o tensor de Ricci; esta última fica

$$
\operatorname{Riem}(\bar{g})_{a c b d}=C(\bar{g})_{a c b d}+2 \bar{g}_{a[b} S(\bar{g})_{c] d}-2 \bar{g}_{c[b} S(\bar{g})_{d] a},
$$

enquanto que (2.6) vira

$$
S(\bar{g})_{a b}=\frac{1}{z} K_{a b}
$$

Combinando (2.9), (2.20) e (2.21), temos:

$$
C(\bar{g})_{a c b d} Z^{c} Z^{d}=\left(\partial_{z}-\frac{1}{z}\right) K_{a b}-K_{a}^{c} K_{c b} .
$$

Usando a expansão de $\bar{g}^{(0)}(z)(2.13)$ e denotando por $K_{a b}^{(j)} \doteq-\frac{j+1}{2} \bar{g}^{(j+1)}$ o $j$-ésimo coeficiente da expansão correspondente para $K_{a b}$, obtemos os coeficientes da expansão de $C(\bar{g})_{a c b d} Z^{c} Z^{d}$ ao redor de $z=0$ :

$$
\left(C(\bar{g})_{a c b d} Z^{c} Z^{d}\right)^{(j)}=j K_{a b}^{(j+1)}+\sum_{m=0}^{j} K_{a}^{(j-m) c} K_{c b}^{(m)} .
$$

(2.23) mostra explicitamente que a informação perdida pelo truncamento das relações de recursão $(2.14)$ em $j=d-1$, referente à parte de traço zero de $\bar{g}_{a b}^{(d-1)}$, está contida em $\left(C(\bar{g})_{a c b d} Z^{c} Z^{d}\right)^{(d-3)}$. De fato, se somos capazes de obter $\bar{g}_{a b}^{(d-1)}$ por outros meios, podemos prosseguir em ordem superior e determinar completamente os coeficientes da expansão (2.14). Assim, podemos dizer que os dados iniciais "corretos" para (2.10) são $\left(\bar{g}^{(0)}, \bar{g}^{(d-1)}\right)$.

Vamos agora nos restringir à classe de métricas AAdS, i.e., $\bar{g}^{(0)}$ é a métrica de $E S U_{d-1}$. Neste caso, os coeficientes $\bar{g}^{(j)}, j<d-1$, devem coincidir, após uma escolha adequada de coordenadas em $\mathscr{I}$, com os da métrica do completamento conforme de $A d S_{d}$ (1.15). Assim, uma métrica AAdS geral tem em $\mathscr{U}_{\epsilon}$, após normalização da constante cosmológica, a forma

$$
d s^{2}=\frac{1}{z^{2}}\left[-\left(1+\frac{z^{2}}{4}\right)^{2} d t^{2}+d z^{2}+\left(1-\frac{z^{2}}{4}\right)^{2} d \Omega_{d-2}^{2}+O\left(z^{d-1}\right)\right]
$$

Temos assim a partir de (2.20), (2.21) e (2.24) a seguinte propriedade grosseira de "peeling" para o tensor de WEYL: $C(\bar{g})_{a b c d}^{(j)}=0$ para $j<d-3$, e, devido à equação de CodAzZi-Mainardi (A.4) (página 109), $\left(\bar{g}^{(0)}(.)_{a}^{e} \bar{g}^{(0)}(.)_{b}^{f} \bar{g}^{(0)}(.)_{c}^{h} Z^{d} C(\bar{g})_{a b c d}\right)^{(j)}=0$ para $j \leq d-3$. Em $A d S_{d}, d \geq 6$, sabemos que $\bar{g}_{a b}^{(d-1)}=0$ e, como $A d S_{d}$ é uma forma espacial, 
$C(\bar{g})_{a b c d}=0$. Portanto, a soma no segundo membro de (2.23) também se anula. Como ela é a mesma para todas as métricas AAdS e todo $j \leq d-3$, ela se anula em todos esses casos. Assim,

$$
K_{a b}^{(d-2)}=\frac{1}{d-3}\left(C(\bar{g})_{a c b d} Z^{c} Z^{d}\right)^{(d-3)}, \forall d \geq 6 .
$$

No caso $d=4$, a soma no segundo membro de $(2.23)$ se reduz a $2 K_{a}^{(1) c} K_{c b}^{(0)}=0$, e em $d=5$, a $K_{a}^{(1) c} K_{c b}^{(1)}=\frac{1}{8} \bar{g}_{a b}^{(0)}$, em virtude de (2.15-2.17). Assim, concluímos que

$$
\bar{g}^{(d-1)}=\left\{\begin{array}{lr}
-\frac{2}{d-1} E_{a b} & (d=4 \text { ou } d \geq 6) \\
-\frac{1}{2} E_{a b}+\frac{1}{16} \bar{g}_{a b}^{(0)} & (d=5)
\end{array},\right.
$$

onde $E_{a b}=\frac{1}{d-3} \lim _{z \backslash 0} z^{3-d} C(\bar{g})_{a c b d} Z^{c} Z^{d}$ é a parte elétrica rescalonada do tensor de WEYL de $\bar{g}$ (o limite existe e é $\mathscr{C}^{\infty}$ pois vimos que os coeficientes de ordem inferior a $d-3$ da expansão de $C(\bar{g})_{a c b d} Z^{c} Z^{d}$ se anulam), o que deixa uma métrica AAdS geral satisfazendo (2.3) na forma

$$
\begin{aligned}
\bar{g}^{(0)}(z)= & -\left(1+\frac{z^{2}}{4}\right)^{2} d t^{2}+d z^{2}+\left(1-\frac{z^{2}}{4}\right)^{2} d \Omega_{d-2}^{2}+ \\
& +\left\{\begin{array}{lr}
-\frac{2}{d-1} E_{a b} z^{d-1} & (d=4 \text { ou } d \geq 6) \\
\left(-\frac{1}{2} E_{a b}+\frac{1}{16} \bar{g}_{a b}^{(0)}\right) z^{4} & (d=5)
\end{array}\right\}+O\left(z^{d}\right) .
\end{aligned}
$$

OBSERVAÇÃo 2.1 O problema da convergência das expansões (2.18-2.19) para $z<\epsilon$ suficientemente pequeno foi afirmativamente resolvido, no caso de $\bar{g}_{a b}^{(0)}$ e $E_{a b}$ realanalíticos, por KICHENASSAMY [Kic04]. No caso não-analítico, não é possível, no presente estado da arte, proceder por meio de aproximações analíticas, por falta de estimativas suficientemente fortes da solução em termos destes dados de fronteira. Isso, numa primeira impressão, era de se esperar, pois o problema de CAUCHY para equações hiperbólicas é sabidamente mal posto para dados iniciais em hipersuperfícies tipo tempo [Had03]. Todavia, tal intuição é possivelmente apenas parcialmente correta, pois os dados iniciais "de verdade" para nosso problema (i.e., $\bar{g}_{a b}^{(0)}$ e $E_{a b}$ ) o tornam consideravelmente diferente do problema de CAUCHY usual para equações hiperbólicas de segunda ordem (lembrar que $\partial_{z} \bar{g}^{(0)}(z) \uparrow_{z=0}=0$ !) - de fato, estimativas com peso envolvendo o tensor de WEYL exercem um papel fundamental na prova da estabilidade não-linear global do espaço-tempo de Minkowski [CK93]. Resultados parciais nesta direção podem ser encontrados em [And06c, And05, And06b, And06a] e nas referências ali citadas. Assim, nos casos não-analiticos, devemos entender as expansões (2.18-2.19) apenas com expansões assintóticas à medida que $z \searrow 0$.

Notar, finalmente, que, em virtude do caráter puramente local da análise feita acima, ela permanece válida sem nenhuma alteração para um domínio de POINCARÉ qualquer 
de um espaço-tempo AAdS e, mais em geral, para qualquer espaço-tempo localmente AAdS (toda a discussão precedendo a fórmula (2.24) é válida até mesmo para espaçostempos localmente tipo AdS gerais). No caso de domínios de Poincaré, devemos substituir as coordenadas (1.6) pelas coordenadas (1.8), e a métrica (1.15), pela métrica (1.9) multiplicada por $z^{2}$. Uma vantagem desta carta no presente contexto é que $\bar{g}_{a b}^{(j)}=0$ para $1 \leq j \leq d-2$, de onde segue que $\bar{g}_{a b}^{(d-1)}=\frac{2}{d-1} E_{a b}$ para todo $d \geq 4$, ao contrário de (2.26). A forma assintótica de uma métrica AAdS na vizinhança de um domínio de Minkowski fica então

$$
\bar{g}^{(0)}(z)_{a b}=\left(\eta_{a b}-\frac{2}{d-1} z^{d-1} E_{a b}\right)+O\left(z^{d}\right) .
$$

\section{3 "Termodinâmica" gravitacional em cunhas AAdS}

Mostraremos como associar a cada cunha $\mathscr{W}_{p, q}$ de um espaço-tempo localmente AAdS $(\mathscr{M}, g), p, q \in \mathscr{D}(\mathscr{I})$, um fluxo de difeomorfismos que se estendem a $\mathscr{D}_{p, q}$ de maneira tal que sua ação coincide nesta região com a ação do subgrupo uniparamétrico (1.26). Neste caso, segue que estes difeomorfismos são isometrias assintóticas, i.e., se $T^{a}$ é o campo vetorial tangente ao fluxo, então $£_{T} g(x, z)=o(z)$ à medida que $z \searrow 0$.

A construção que faremos é um caso particular de um procedimento mais geral, aplicável a qualquer diamante $\mathscr{O}_{p, q}=I^{+}(p) \cap I^{-}(q)$ relativamente compacto num espaçotempo causalmente simples $(\mathscr{M}, g)$, aparentado ao método de Geroch [Ger70] para a construção de funções tempo globais em espaços-tempos globalmente hiperbólicos.

\subsubsection{Evolução temporal geométrica em diamantes}

Mostraremos inicialmente como associar a cada diamante $\mathscr{D}_{p, q}$ no infinito conforme de um espaço-tempo localmente $\operatorname{AAdS}(\mathscr{M}, g), p, q \in \mathscr{D}(\mathscr{I})$ uma função tempo global associada à ação do subgrupo uniparamétrico (1.26).

TeOrema 2.2 Considere um espaço-tempo AAdS d-dimensional $(\mathscr{M}, g), r \in \mathscr{I} e$ $p_{0}, q_{0} \in \mathscr{M} i n(r)$ tais que, se $\mathscr{M} i n(r) \ni p \mapsto x^{\mu}(p)$ é a carta global do espaço-tempo de Minkowski, temos que $x^{\mu}\left(p_{0}\right)=(-1,0)$ e $x^{\mu}\left(q_{0}\right)=(1,0)$. Considere o grupo uniparamétrico $u_{p_{0}, q_{0}}^{\lambda}$ de difeomorfismos conformes (1.26) de $\left(\mathscr{D}_{p_{0}, q_{0}}, \eta\left\lceil\mathscr{D}_{p_{0}, q_{0}}\right)\right.$, dado para $p$ no plano $x^{0}-x^{1}$ por

$$
x^{ \pm}\left(u_{p_{0}, q_{0}}^{\lambda}(p)\right) \doteq \frac{\left(1+x^{ \pm}\right)-e^{-\lambda}\left(1-x^{ \pm}\right)}{\left(1+x^{ \pm}\right)+e^{-\lambda}\left(1-x^{ \pm}\right)}, \lambda \in \mathbb{R}
$$

$\left(x^{ \pm}=x^{0} \pm x^{1}\right.$ são as coordenadas de cone de luz no plano $\left.x^{0}-x^{1}\right)$ e estendido para $p \in \mathscr{D}_{p_{0}, q_{0}}$ por rotações espaciais. Defina a seguinte folheação $\lambda \mapsto \Sigma_{p_{0}, q_{0}}^{\lambda}$ de $\mathscr{D}_{p_{0}, q_{0}}$ por superficies de CAUCHY: 
- $\Sigma_{p_{0}, q_{0}} \doteq \Sigma_{p_{0}, q_{0}}^{0}=\mathscr{D}_{p_{0}, q_{0}} \cap\left\{x^{0}=0\right\}$;

- $\Sigma_{p_{0}, q_{0}}^{\lambda}=u_{p_{0}, q_{0}}^{\lambda}\left(\Sigma_{p_{0}, q_{0}}^{0}\right)$, i.e., a folheação é dada pelo difeomorfismo $F_{p_{0}, q_{0}}: \mathbb{R} \times$ $\Sigma_{p_{0}, q_{0}} \ni(\lambda, p) \mapsto u_{p_{0}, q_{0}}^{\lambda}(p) \in \mathscr{D}_{p_{0}, q_{0}}$.

- $\lambda$ é, portanto, a função tempo global associada a $F_{p_{0}, q_{0}}$.

Então,

$$
\lambda=\frac{1}{d-1} \log \left[\frac{\left|\mathscr{D}_{p_{0}, u_{p_{0}, q_{0}}^{\lambda}(p)}\right|}{\left|\mathscr{D}_{u_{p_{0}, q_{0}}(p), q_{0}}\right|}\right]=\log \left[\frac{d_{\eta}\left(p_{0}, u_{p_{0}, q_{0}}^{\lambda}(p)\right)}{d_{\eta}\left(u_{p_{0}, q_{0}}^{\lambda}(p), q_{0}\right)}\right], \forall p \in \Sigma_{p_{0}, q_{0}}
$$

onde $d_{\eta}$ é a distância Lorentziana associada a $\eta$ (ver Apêndice A). Lembrar que a medida de LEBESGUE é precisamente a medida induzida pelo elemento de volume $\sqrt{|\eta|}$ (notamos por conveniência que $\left|\mathscr{D}_{p_{0}, q_{0}}\right|=2 \int_{0}^{1}\left|B_{r}(0)\right| d r=2 \int_{0}^{1} \frac{r^{d-2}}{d-2} \operatorname{Vol}^{d-3} d r=$ $\left.\frac{2}{(d-1)(d-2)} \mathrm{Vol} S^{d-3}\right)$.

Prova. Devido à simetria rotacional de $u_{p_{0}, q_{0}}^{\lambda}$, podemos restringir nossas considerações ao plano $x^{0}-x^{1}$. Neste caso, escrevamos $x^{\mu}(p)=\left(0, x^{1}, 0, \ldots, 0\right)$, $x^{1} \in[-1,1]$, de onde segue que

$$
x^{ \pm}\left(u_{p_{0}, q_{0}}^{\lambda}(p)\right)=\frac{\left(1 \pm x^{1}\right)-e^{-\lambda}\left(1 \mp x^{1}\right)}{\left(1 \pm x^{1}\right)+e^{-\lambda}\left(1 \mp x^{1}\right)}
$$

e, portanto,

$$
\begin{aligned}
x^{0}\left(u_{p_{0}, q_{0}}^{\lambda}(p)\right) & =\frac{1}{2}\left(x^{+}\left(u_{p_{0}, q_{0}}^{\lambda}(p)\right)+x^{-}\left(u_{p_{0}, q_{0}}^{\lambda}(p)\right)\right)= \\
& =\frac{\left(1-\left(x^{1}\right)^{2}\right)\left(1-e^{-2 \lambda}\right)}{\left(1-\left(x^{1}\right)^{2}\right)\left(1+e^{-2 \lambda}\right)+2 e^{-\lambda}\left(1+\left(x^{1}\right)^{2}\right)}
\end{aligned}
$$

$\mathrm{e}$

$$
\begin{aligned}
x^{1}\left(u_{p_{0}, q_{0}}^{\lambda}(p)\right) & =\frac{1}{2}\left(x^{+}\left(u_{p_{0}, q_{0}}^{\lambda}(p)\right)-x^{-}\left(u_{p_{0}, q_{0}}^{\lambda}(p)\right)\right)= \\
& =\frac{4 x^{1} e^{-\lambda}}{\left(1-\left(x^{1}\right)^{2}\right)\left(1+e^{-2 \lambda}\right)+2 e^{-\lambda}\left(1+\left(x^{1}\right)^{2}\right)}
\end{aligned}
$$

Seja o ponto $q=(t, \mathbf{0}), t \in(-1,1)$. O diamante $\mathscr{D}_{p_{0}, q}$ é uma translação de $\frac{1+t}{2} \mathscr{D}_{p_{0}, q_{0}}$, e o diamante $\mathscr{D}_{q, q_{0}}$ é uma translação de $\frac{1-t}{2} \mathscr{D}_{p_{0}, q_{0}}-\operatorname{logo}$, temos que $\left|\mathscr{D}_{p_{0}, q}\right|=\left(\frac{1+t}{2}\right)^{d-1}\left|\mathscr{D}_{p_{0}, q_{0}}\right|$ e $\left|\mathscr{D}_{q, q_{0}}\right|=\left(\frac{1-t}{2}\right)^{d-1}\left|\mathscr{D}_{p_{0}, q_{0}}\right|$. Mais em geral, se $q=$ $(t, r, 0, \ldots, 0)$, existe um "boost" de Lorentz no plano $x^{0}-x^{1}$ ao redor de $p_{0}$ que leva $\mathscr{D}_{p_{0}, q}$ em $\mathscr{D}_{p_{0}, q^{+}}$, onde $q^{+}=\left(\left((1+t)^{2}-r^{2}\right)^{\frac{1}{2}}-1, \mathbf{0}\right)$, e um "boost" de Lorentz no plano $x^{0}-x^{1}$ ao redor de $q_{0}$ que leva $\mathscr{D}_{p_{0}, q}$ em $\mathscr{D}_{q^{-}, q_{0}}$, onde 
$q^{-}=\left(1-\left((1-t)^{2}-r^{2}\right)^{\frac{1}{2}}, \mathbf{0}\right)$. Como transformações de LoREntz preservam volume, temos que

$$
\left|\mathscr{D}_{p_{0}, q}\right|=\left(\frac{\left((1+t)^{2}-r^{2}\right)^{\frac{1}{2}}}{2}\right)^{d-1}\left|\mathscr{D}_{p_{0}, q_{0}}\right|
$$

e

$$
\left|\mathscr{D}_{q, q_{0}}\right|=\left(\frac{\left((1-t)^{2}-r^{2}\right)^{\frac{1}{2}}}{2}\right)^{d-1}\left|\mathscr{D}_{p_{0}, q_{0}}\right| .
$$

Finalmente, tomando $q=q(\lambda)=u_{p_{0}, q_{0}}^{\lambda}(p)$, segue que

$$
\left(1+x^{0}(q(\lambda))\right)^{2}-x^{1}(q(\lambda))^{2}=\frac{4\left(1-\left(x^{1}\right)^{2}\right)}{\left(1-\left(x^{1}\right)^{2}\right)\left(1+e^{-2 \lambda}\right)+2 e^{-\lambda}\left(1+\left(x^{1}\right)^{2}\right)}
$$

e

$$
\left(1-x^{0}(q(\lambda))\right)^{2}-x^{1}(q(\lambda))^{2}=\frac{4 e^{-2 \lambda}\left(1-\left(x^{1}\right)^{2}\right)}{\left(1-\left(x^{1}\right)^{2}\right)\left(1+e^{-2 \lambda}\right)+2 e^{-\lambda}\left(1+\left(x^{1}\right)^{2}\right)},
$$

e daí a fórmula (2.29). A segunda identidade segue do fato de que $\left|\mathscr{D}_{p, q}\right|=$ $\frac{1}{2^{d-2}(d-1)(d-2)} \operatorname{Vol} S^{d-3} d_{\eta}(p, q)^{d-1}$.

A importância do Teorema (2.2) ficará clara em breve.

É importante ter em mente que, em espaços-tempos fortemente causais $d$-dimensionais $(\mathscr{M}, g)$, a distância Lorentziana $d_{g}$ coincide com a distância geodésica em vizinhanças normais convexas (ver a fórmula 2.36, página 45, e a discussão que se segue para mais detalhes). Definamos, para tais espaços-tempos, a medida $\mu_{d_{g}}$ através dos seguintes passos:

1. Para um diamante (aberto e relativamente compacto) $\mathscr{O}_{p, q} \doteq I^{+}(p) \cap I^{-}(q), p \ll q$, seja

$$
\mu_{d_{g}}\left(\mathscr{O}_{p, q}\right) \doteq \frac{1}{2^{d-1} d(d-1)} \operatorname{Vol} S^{d-2} d_{g}(p, q)^{d}
$$

2. Considere uma métrica Riemanniana auxiliar $h$ em $\mathscr{M}$, definindo uma distância Riemanniana $d_{h}$ (i.e., $\left(\mathscr{M}, d_{h}\right)$ é um espaço métrico, com a mesma topologia de $\mathscr{M})$. Todo $\mathscr{U} \subset \mathscr{M}$ pode ser recoberto por uma coleção enumerável de $\mathscr{O}_{p, q}$ 's satisfazendo $\operatorname{diam}_{d_{h}} \mathscr{O}_{p, q} \doteq \sup \left\{d_{h}(r, s): r, s \in \mathscr{O}_{p, q}\right\} \leq \frac{1}{n}$ para qualquer $n \in \mathbb{Z}_{+}$, em virtude da $\sigma$-compacidade de $\mathscr{M}$. Portanto,

$$
\mu_{d_{g}}^{*, n}(\mathscr{U}) \doteq \inf \left\{\sum_{i=1}^{\infty} \mu_{d_{g}}\left(\mathscr{O}_{p_{i}, q_{i}}\right): p_{i} \ll q_{i} \in \mathscr{M}, \bigcup_{n=1}^{\infty} \mathscr{O}_{p_{i}, q_{i}} \supset \mathscr{U}, \operatorname{diam}_{d_{h}} \mathscr{O}_{p_{i}, q_{i}} \leq \frac{1}{n}\right\}
$$


define uma medida exterior no conjunto das partes $P(\mathscr{M})$ de $\mathscr{M}$ para todo $n$, i.e., $\mu_{d_{g}}^{*, n} \geq 0, \mu_{d_{g}}^{*, n}(\varnothing)=0$ e $\mu_{d_{g}}^{*, n}\left(\bigcup_{i=1}^{\infty} \mathscr{U}_{i}\right) \leq \sum_{i=1}^{\infty} \mu_{d_{g}}^{*, n}\left(\mathscr{U}_{i}\right)$, para todo $\mathscr{U}_{i} \subset \mathscr{M}$. Como $\mu_{d_{g}}^{*, n+1}(\mathscr{U}) \geq \mu_{d_{g}}^{*, n}(\mathscr{U})$, segue então que $\mu_{d_{g}}^{*}(\mathscr{U}) \doteq \lim _{n \rightarrow \infty} \mu_{d_{g}}^{*, n}(\mathscr{U})$ existe (embora possa ser igual a $+\infty$ ) e define uma medida exterior métrica, i.e., se $\operatorname{dist}_{d_{h}}(\mathscr{U}, \mathscr{V}) \doteq \inf \left\{d_{h}(r, s): r \in \mathscr{U}, s \in \mathscr{V}\right\}>0$, então $\mu_{d_{g}}^{*}(\mathscr{U} \cup \mathscr{V})=\mu_{d_{g}}^{*}(\mathscr{U})+$ $\mu_{d_{g}}^{*}(\mathscr{V})$.

3. Pelo Teorema de Extensão de CARAThÉOdory [Mun71], a coleção de conjuntos $\mu_{d_{g}}$ mensuráveis

$$
\mathscr{P}_{d_{g}}(\mathscr{M}) \doteq\left\{\mathscr{U} \subset \mathscr{M}: \mu_{d_{g}}^{*}(\mathscr{V})=\mu_{d_{g}}^{*}(\mathscr{V} \cap \mathscr{U})+\mu_{d_{g}}^{*}(\mathscr{V} \backslash \mathscr{U})\right\}
$$

constitui uma $\sigma$-álgebra de conjuntos, i.e., $\varnothing, \mathscr{M} \in \mathscr{P}_{d_{g}}(\mathscr{M}), \mathscr{U} \in \mathscr{P}_{d_{g}}(\mathscr{M}) \Rightarrow$ $\mathscr{M} \backslash \mathscr{U} \in \mathscr{P}_{d_{g}}(\mathscr{M})$ e $\mathscr{U}_{n} \in \mathscr{P}_{d_{g}}(\mathscr{M}), \forall n \in \mathbb{Z}_{+} \Rightarrow \bigcup_{n=1}^{\infty} \mathscr{U}_{n} \in \mathscr{P}_{d_{g}}(\mathscr{M})$, e $\mu_{d_{g}}^{*}\left\lceil\mathscr{P}_{d_{g}}(\mathscr{M})\right.$ é uma medida, i.e., satisfaz $\mu_{d_{g}}^{*}\left(\bigcup_{n=1}^{\infty} \mathscr{U}_{n}\right)=\sum_{n=1}^{\infty} \mu_{d_{g}}^{*}\left(\mathscr{U}_{n}\right)$, para toda seqüência $\left\{\mathscr{U}_{n}\right\}$ de elementos mutualmente disjuntos de $\mathscr{P}_{d_{g}}(\mathscr{M})$. Mais ainda, em razão da metricidade de $\mu_{d_{g}}^{*}, \mathscr{P}_{d_{g}}(\mathscr{M})$ inclui todos os conjuntos fechados de $\mathscr{M}$ e, portanto, todos os conjuntos Borelianos de $\mathscr{M}$ - em particular, $\mathscr{O}_{p, q} \in \mathscr{P}_{d_{g}}(\mathscr{M})$ para todo $p \ll q \in \mathscr{M}$. Assim, estendemos a notação e denotamos por $\mu_{d_{g}}$ a restrição de $\mu_{d_{g}}^{*}$ aos conjuntos Borelianos de $\mathscr{M}$.

4. Se $\mu_{g}$ é a medida Boreliana associada ao elemento de volume $\sqrt{|g|} d x$, pode-se mostrar [GS07], empregando as fórmulas (A.4)-(A.6), que

$$
\mu_{g}\left(\mathscr{O}_{p, q}\right)=\mu_{d_{g}}\left(\mathscr{O}_{p, q}\right)\left(1+O\left(d_{g}(p, q)^{2}\right)\right), \forall p \ll q \in \mathscr{M},
$$

de onde segue que $\mu_{d_{g}}$ é absolutamente contínua com respeito a $\mu_{g}$ [Rud87], e, portanto, a derivada de RADON-NIKÓDYM de $\mu_{d_{g}}$ com respeito a $\mu_{g}$

$$
\frac{d \mu_{d_{g}}}{d \mu_{g}}(r)=\lim _{\substack{p \ll r \ll q, d_{g}(p, r), d_{g}(r, q) \backslash 0}} \frac{\mu_{d_{g}}\left(\mathscr{O}_{p, q}\right)}{\mu_{g}\left(\mathscr{O}_{p, q}\right)}=1 .
$$

Suponhamos agora que $(\mathscr{M}, g)$ é causalmente simples, de modo que todo diamante $\mathscr{O}_{p, q}$ não-vazio e relativamente compacto é globalmente hiperbólico, cujas superfícies de CAUCHY $\mathscr{S}_{p, q}$ são conjuntos acausais com bordo igual a $\partial I^{+}(p) \cap \partial I^{-}(q)$. Note, contudo, que se $\overline{\mathscr{O}_{p, q}}$ não está contido em nenhuma vizinhança geodesicamente convexa, então $\mathscr{O}_{p, q}$ $n \tilde{a} o$ é igual a $D\left(\mathscr{S}_{p, q}\right)$ em $\mathscr{M}$, pois neste caso $\partial \mathscr{O}_{p, q}=\left(\partial I^{+}(p) \cap J^{-}(q)\right) \cup\left(\partial I^{-}(q) \cap J^{+}(p)\right)$ possui segmentos geodésicos com pares de pontos conjugados, ou seja, há pontos em $\partial \mathscr{O}_{p, q}$ que pertencem a geodésicas nulas que deixam de ser acronais antes de atingir 0 bordo de $\mathscr{S}_{p, q}$. Assim, temos que $\operatorname{int} D\left(\mathscr{S}_{p, q}\right) \supsetneqq \mathscr{O}_{p, q}$ neste caso (a igualdade só ocorre se os segmentos geodésicos nulos que geram $\partial \mathscr{O}_{p, q}$ são acronais, como por exemplo no 
caso em que $\overline{\mathscr{O}_{p, q}}$ está contido em alguma vizinhança geodesicamente convexa).

Não obstante, se $\mathscr{O}_{p, q}$ é relativamente compacto, mesmo que não geodesicamente convexo, podemos aplicar o argumento de GEROCH [Ger70, HE73], complementado pelas considerações de DiECKMANN [Die88], para construir uma folheação contínua de $\mathscr{O}_{p, q}$ por superfícies de CAUCHY a partir de $\mu_{d_{g}}$. Vamos delinear a construção no presente caso empregando as propriedades de $d_{g}$ no caso em que $(\mathscr{M}, g)$ é causalmente simples, até porque veremos que a função tempo global que acabaremos por construir possui regularidade maior que a construída por $\mathrm{GEROCH}$, independentemente do tamanho de $\mathscr{O}_{p, q}$.

A propriedade que usaremos é a desigualdade triangular reversa (ver Apêndice A, página 115)

$$
p \leq r \leq q \Rightarrow d_{g}(p, q) \geq d_{g}(p, r)+d_{g}(r, q) .
$$

Segue desta desigualdade que, se $\gamma:[0,1] \rightarrow \mathscr{M}$ é uma curva causal inextensível em $\mathscr{O}_{p, q}$, então $\lambda \mapsto d_{g}(\gamma(\lambda), q)$ (resp. $\lambda \mapsto d_{g}(\underline{p, \gamma}(\lambda))$ ) é uma função limitada por $d_{g}(p, q)\left(<+\infty\right.$ em virtude da compacidade de $\overline{\mathscr{O}_{p, q}}$ e da Definição A.1 de distância Lorentziana) e estritamente decrescente (resp. crescente) de $\lambda$-lembrar que toda curva causal maximal (i.e., cujo comprimento de arco entre dois pontos quaisquer é igual à distância Lorentziana) é necessariamente uma geodésica, a menos de reparametrização. Assim, as superfícies de nível de $d_{g}(., q)$ e $d_{g}(p,$.$) são acausais para valores diferentes de$ zero. Mais ainda, $d_{g}(p,$.$\left.) (resp. d_{g}(., q)\right)$ tende a zero ao longo de qualquer curva causal inextensível passada (resp. futura) em $\mathscr{O}_{p, q}$. Empregando as propriedades obtidas acima, podemos repetir a demonstração da Proposição 3.1 em [AGH98b] no nosso contexto, obtendo a seguinte

Proposição 2.3 ([AGH98b]) $d_{g}(p,$.$) e d_{g}(., q)$ são semi-convexas, i.e., para cada $r \in$ $\mathscr{O}_{p, q}$ existe uma vizinhança de $\mathscr{U} \ni r$, uma carta local $x: \mathscr{U} \rightarrow \mathbb{R}^{d}$ e $f \in \mathscr{C}^{\infty}(\mathscr{U})$ tal que $\left(d_{g}(p,.) \Gamma_{\mathscr{U}}+f\right) \circ x^{-1} e\left(d_{g}(., q) \Gamma_{\mathscr{U}}+f\right) \circ x^{-1}$ s $\tilde{a} o$ convexas em $x(\mathscr{U})$.

Na verdade, o resultado que obtemos é o seguinte: existem $\phi_{p, r}, \phi_{r, q} \in \mathscr{C}^{\infty}(\mathscr{U}$ tais que $d_{g}(p, r)=\phi_{p, r}(r)$ e $d_{g}(r, q)=\phi_{r, q}(r), d_{g}(p,.) \geq \phi_{p, r}$ e $d_{g}(., q) \geq \phi_{r, q}$ em $\mathscr{U}$ e as Hessianas $D^{2} \phi_{p, r}$ e $D^{2} \phi_{r, q}$ são tais que $D^{2} \phi_{p, r}(r)-c_{p, r} \mathbb{1}$ e $D^{2} \phi_{r, q}(r)-c_{r, q} \mathbb{1}$ são matrizes positivas semi-definidas para $c_{p, r}, c_{r, q} \in \mathbb{R}$, o que não só implica em semiconvexidade no sentido da Proposição 2.3 [AGH98a], como também garante que a definição dada é independente de coordenadas. Nestas circunstâncias, podemos invocar o resultado clássico de Alexandrov, que nos diz que uma função convexa não só é localmente LiPSCHITz, mas também é duas vezes diferenciável quase em toda parte com respeito à medida de LEBESGUE (ver [EG92] para uma demonstração deste fato). Tal resultado obviamente se estende a funções semi-convexas. Como a restrição de 
$\mu_{g}\left(\mathrm{e}\right.$, portanto,$\left.\mu_{d_{g}}\right)$ a vizinhanças normais é absolutamente contínua com respeito à medida de LEBESGUE, obtemos destarte a seguinte

Proposição $2.4 d_{g}(p,$.$) e d_{g}(., q)$ são localmente Lipschitzianas e duas vezes diferenciáveis quase em toda a parte.

Podemos finalmente definir nossa função tempo global em $\mathscr{O}_{p, q}$ associada a $d_{g}$ :

$$
\lambda_{p, q}^{g}(r) \doteq \log \left[\frac{d_{g}(p, r)}{d_{g}(r, q)}\right]=\frac{1}{d} \log \left[\frac{\mu_{d_{g}}\left(\mathscr{O}_{p, r}\right)}{\mu_{d_{g}}\left(\mathscr{O}_{r, q}\right)}\right],
$$

onde a segunda igualdade dá a relação entre a $\lambda_{p, q}^{g}$ e a função tempo global de GEROCH em $\mathscr{O}_{p, q}$ associada a $\mu_{d_{g}}$ (a normalização da medida não tem conseqüência alguma). Que $\left(\lambda_{p, q}^{g}\right)^{-1}(t)$ é uma superfície de CAUCHY para todo $t \in \mathbb{R}$, segue da (única) Proposição em [Die88].

Mostraremos agora o comportamento assintótico de $\lambda_{p, q}^{g}(r)$ no caso em que $\mathscr{O}_{p, q}$ é contido numa vizinhança geodesicamente convexa, à medida que

(i) $d_{g}(p, r) \rightarrow 0$ mantendo $d_{g}(r, q) \neq 0$, e

(ii) $d_{g}(r, q) \rightarrow 0$ mantendo $d_{g}(p, r) \neq 0$.

A restrição sobre o tamanho de $\mathscr{O}_{p, q}$ garante que $d_{g}(p,),. d_{g}(., q)$ e, portanto, $\lambda_{p, q}^{g}$ são $\mathscr{C}^{\infty}$, mas ressaltamos que as fórmulas que se seguem dependem apenas de derivadas de ordem $\leq 2$ de $d_{g}(p,$.$) e d_{g}(., q)$. Discutiremos mais adiante outras conseqüências desta hipótese.

Seja $\mathscr{U}$ uma vizinhança geodesicamente convexa. Definamos então a função-mundo de SYNGE

$$
\Gamma_{g} \mathscr{U} \times \mathscr{U} \ni(p, q) \mapsto \Gamma_{g}(p, q) \doteq-\frac{1}{2} \int_{0}^{1} g\left(\dot{\gamma}_{p, q}(s), \dot{\gamma}_{p, q}(s)\right) d s,
$$

onde $\gamma_{p, q}:[0,1] \rightarrow \mathscr{U}$ é o (único) segmento geodésico ligando $p=\gamma_{p, q}(0)$ e $q=\gamma_{p, q}(1)$. $\Gamma_{g}$ possui as seguintes propriedades (ver [Fri75] para as demonstrações):

- $\Gamma_{g} \in \mathscr{C}^{\infty}\left(\bigcup_{p \in \mathscr{M}}\{p\} \times \mathscr{U}_{p}\right)$, onde $\mathscr{U}_{p}$ é uma vizinhança aberta e geodesicamente convexa de $p$;

- $\Gamma_{g}(p, q)=\Gamma_{g}(q, p)$; 
- $\nabla^{a} \Gamma_{g}(p,)=.-\dot{\gamma}_{p, .}^{a}($.$) e \nabla^{a} \Gamma_{g}(., q)=-\dot{\gamma}_{., q}^{a}($.$) , onde . denota a variável onde age \nabla$. Segue imediatamente a fórmula fundamental

$$
g^{-1}\left(d_{p} \Gamma_{g}(p, q), d_{p} \Gamma_{g}(p, q)\right)=g^{-1}\left(d_{q} \Gamma_{g}(p, q), d_{q} \Gamma_{g}(p, q)\right)=-2 \Gamma_{g}(p, q),
$$

onde $d_{p}$ e $d_{q}$ denotam respectivamente a diferencial com respeito à primeira e segunda variáveis.

- $\left(\nabla_{a} \nabla_{b} \Gamma_{g}(p,).\right)(p)=-g_{a b}(p)$.

Primeiramente, devemos notar que $d_{g}$ e $2\left(\Gamma_{g}\right)^{\frac{1}{2}}$ coincidem em $\mathscr{U} \times J^{+}(\mathscr{U}, \mathscr{U})$, pois $(\mathscr{M}, g)$ é causalmente simples por hipótese e, portanto, fortemente causal (ver Teorema 4.27 em [BEE96]). ( $\left.\Gamma_{g}\right)^{\frac{1}{2}}$ é contínua mas não-diferenciável em $d_{g}^{-1}(0) \cap\left(\mathscr{U} \times J^{+}(\mathscr{U}, \mathscr{U})\right.$, em virtude da presença da raiz quadrada. Assim, empregaremos a fórmula equivalente

$$
\lambda_{p, q}^{g}(r)=\frac{1}{2}\left(\log \left(d_{g}(p, r)^{2}\right)-\log \left(d_{g}(r, q)^{2}\right)\right)=\frac{1}{2}\left(\log \left(\Gamma_{g}(p, r)\right)-\log \left(\Gamma_{g}(r, q)\right)\right)
$$

ao invés de (2.35) no que se segue. Assumimos, de agora em diante, $p$ e $q$ fixos e $\Gamma_{g}(p, r)$ e $\Gamma_{g}(r, q)$ como funções só de $r$, ou seja, diferenciais e derivadas covariantes atuam apenas em $r$. Desta forma, podemos aliviar um bocado de notação.

Vamos inicialmente obter a forma infinitesimal do fluxo de difeomorfismos induzido pela função tempo global $\lambda_{p, q}^{g}$ :

$$
\nabla_{a} \lambda_{p, q}^{g}(r)=\frac{1}{2}\left(\frac{\nabla_{a} \Gamma_{g}(p, r)}{\Gamma_{g}(p, r)}-\frac{\nabla_{a} \Gamma_{g}(r, q)}{\Gamma_{g}(r, q)}\right) .
$$

Consideremos agora o campo de vetores $T^{a}$ em $\mathscr{O}_{p, q}$ unicamente determinado pelas seguintes condições algébricas:

- $T^{a} \nabla_{a} \lambda_{p, q}^{g}(r)=1$ para todo $r \in \mathscr{O}_{p, q}$;

- $g(T, X)=0$ para todo $X^{a}$ tangente a $\left(\lambda_{p, q}^{g}\right)^{-1}(t), t \in \mathbb{R}$.

Em outras palavras, $T^{a}$ é o campo vetorial que gera o fluxo de difeomorfismos induzido pela folheação de $\mathscr{O}_{p, q}$ pelas hipersuperfícies de nível de $\lambda_{p, q}$. Temos, então, que

$$
g(T, T)=\frac{1}{g^{-1}\left(d \lambda_{p, q}^{g}, d \lambda_{p, q}^{g}\right)}
$$

onde

$$
g^{-1}\left(d \lambda_{p, q}^{g}, d \lambda_{p, q}^{g}\right)=-\frac{1}{2}\left(\frac{1}{\Gamma_{g}(p, r)}+\frac{1}{\Gamma_{g}(r, q)}+\frac{g^{-1}\left(d \Gamma_{g}(p, r), d \Gamma_{g}(r, q)\right)}{\Gamma_{g}(p, r) \Gamma_{g}(r, q)}\right)
$$


Logo,

$$
\begin{aligned}
T^{a} & =\frac{1}{g^{-1}\left(d \lambda_{p, q}^{g}, d \lambda_{p, q}^{g}\right)} \nabla^{a} \lambda_{p, q}^{g}= \\
& =\frac{\Gamma_{g}(p, r) \nabla^{a} \Gamma_{g}(r, q)-\Gamma_{g}(r, q) \nabla^{a} \Gamma_{g}(p, r)}{\Gamma_{g}(p, r)+\Gamma_{g}(r, q)+g^{-1}\left(d \Gamma_{g}(p, r), d \Gamma_{g}(r, q)\right)} .
\end{aligned}
$$

Fica claro a partir de (2.42) que, assintoticamente à medida que $r \rightarrow p$ e $r \rightarrow q$, ou seja, respectivamente $(-) d_{g}(p, r) \searrow 0, d_{g}(r, q) \nearrow d_{g}(p, q)$ e $(+) d_{g}(r, q) \searrow 0$, $d_{g} \nearrow d_{g}(p, q), T^{a}$ aproxima-se de um campo de Killing conforme, i.e.,

$$
\begin{aligned}
\nabla_{a} T_{b}+\nabla_{b} T_{a} & =f(.) g_{a b}+o\left(\min \left\{d_{g}(p, r), d_{g}(r, q)\right\}\right)= \\
& =\frac{2}{d}\left(\nabla_{c} T^{c}\right) g_{a b}+o\left(\min \left\{d_{g}(p, r), d_{g}(r, q)\right\}\right)
\end{aligned}
$$

à medida que $r \rightarrow p$ ou $r \rightarrow q$. Mais precisamente, $T^{a}$ (resp. $-T^{a}$ ) se aproxima de uma dilatação infinitesimal ao redor de $p$ (resp. q). Em coordenadas normais geodésicas $\left(x^{\mu}\right)$ ao redor de $p$ (resp. $q$ ), as componentes de $T^{a}$ ficam

$$
T^{\mu}=x^{\mu}-x^{\mu}(p)+O\left((x-x(p))^{2}\right)\left(\operatorname{resp} . x^{\mu}-x^{\mu}(q)+O\left((x-x(q))^{2}\right)\right) .
$$

\subsubsection{Construção de isometrias assintóticas}

Especializando-nos uma vez mais em espaços-tempos (localmente) tipo $\operatorname{AdS}(\mathscr{M}, g)$, vemos que, devido ao fato de que $\left(\mathscr{I}, \bar{g}^{(0)}\right)$ é totalmente geodésico com respeito a $(\overline{\mathscr{M}}, \bar{g})$ (e, portanto, $\frac{1}{2} d_{\bar{g}}^{2} \Upsilon_{\mathscr{I}^{2} \cap \mathscr{U}^{2}}=\Gamma_{\bar{g}}\left\lceil\mathscr{I}^{2} \cap \mathscr{U}^{2}=\Gamma_{\bar{g}^{(0)}}\left\lceil_{\mathscr{I}^{2} \cap \mathscr{U}^{2}}\right.\right.$, onde $\mathscr{U}$ é uma vizinhança geodesicamente convexa em $\overline{\mathscr{M}}$ - ver Proposição $4.32 \mathrm{em}$ [BEE96]), $\lambda_{p, q}^{\bar{g}} \Upsilon_{\mathscr{I}} \equiv \lambda_{p, q}^{\bar{g}^{(0)}}$ para todo par $p \ll \mathscr{I} q \in \mathscr{I}$ tal que $\overline{\mathscr{W}_{p, q}}$ está contido numa vizinhança geodesicamente convexa em $(\overline{\mathscr{M}}, \bar{g})$, uma vez que $\lambda_{p, q}^{\bar{g}}$ é construída unicamente a partir de $\Gamma_{\bar{g}}$. Em particular, se $(\mathscr{M}, g)$ é (localmente) AAdS, e $x(p)=(-1,0)$ e $x(q)=(1,0)$ em uma carta Cartesiana $x:\left(\mathscr{M} i n(r), \bar{g}^{(0)}\lceil\mathscr{M} i n(r)) \rightarrow\left(\mathbb{R}^{1, d-2}, \eta\right)\right.$ sobre um domínio de Minkowski $\mathscr{M}$ in $(r)$ para algum $r \in \mathscr{I}$, segue que $\lambda_{p, q}^{\bar{g}} \uparrow_{\mathscr{I}} \equiv \lambda$, onde $\lambda$ é dada pela fórmula (2.29), que é a conclusão do Teorema 2.2. Ou seja, $T^{a}$ não só é tangente a $\mathscr{I}$ como $T^{a} \Upsilon_{\mathscr{I}}$ coincide com o campo vetorial em $\mathscr{D}_{p, q}$ gerado pelo subgrupo uniparamétrico $\lambda \mapsto u_{p_{0}, q_{0}}^{\lambda}$ do grupo conforme de $\left(\mathscr{I}, \bar{g}^{(0)}\right)$ dado por (1.23). Estendendo a assertiva do Teorema 2.2 a $(p, q) \in \mathscr{D}(\mathscr{I})$ pelas relações de comutação de $S O_{e}(2, d-1)$ e lembrando que (resp. a componente conexa à identidade d)o grupo conforme é precisamente o conjunto de difeomorfismos que preservam a estrutura causal (resp., mais a orientação e a orientação temporal), obtemos:

Proposição 2.5 Seja $(\mathscr{M}, g)$ um espaço-tempo localmente AAdS, $(p, q) \in \mathscr{D}(\mathscr{I})$ tais que $\overline{\mathscr{W}_{p, q}}$ está contido numa vizinhança geodesicamente convexa em $(\overline{\mathscr{M}}, \bar{g})$. Então $T^{a}$ 
é tangente a $\mathscr{I}$ e $\left.\lambda_{p, q}^{\bar{g}}\right\rceil_{\mathscr{I}}=\lambda_{p, q}^{\bar{g}^{(0)}}=\lambda$, onde $u_{p, q}^{\lambda}$ é o subgrupo de isotropia (1.26) de $\mathscr{D}_{p, q}$ do grupo conforme de $\left(\mathscr{I}, \bar{g}^{(0)}\right)$.

Segue da Proposição 2.5 que $T^{a}$ gera um grupo uniparamétrico de isometrias assintóticas de $\left(\mathscr{W}_{p, q}, g\left\lceil_{\mathscr{W}_{p, q}}\right)\right.$, i.e., $£_{T} g=o(z)$ à medida que $z \searrow 0$.

Convém notar que, no caso $A d S$, vemos a partir da demonstração do Teorema 2.2 e empregando cartas de Poincaré que $T^{a}$ é precisamente o campo de Killing em $\mathscr{W}_{p, q}$ que gera o subgrupo conforme de isotropia deste último, para todo $(p, q) \in \mathscr{D}(\mathscr{I})$. Portanto, nossa construção generaliza naturalmente os campos de Killing de $A d S_{d}$.

O que acontece agora para $\mathscr{W}_{p, q}$ 's "grandes", i.e., tais que $\overline{\mathscr{W}_{p, q}}$ é relativamente compacto em $\overline{\mathscr{M}}$, mas não está contido numa vizinhança geodesicamente convexa em $(\overline{\mathscr{M}}, \bar{g})$ ? Temos problemas de dois tipos, ambos com a mesma origem (a saber, nos pontos críticos da aplicação geodésica), mas com conseqüências diferentes, mais ou menos graves para nós:

1. $L_{g}$ deixa de ser bem definida, e $d_{g}$ deixa de ser $\mathscr{C}^{\infty}$ em $d_{g}(p, .)^{-1}\left(\left(0, d_{g}(p, q)\right)\right)$ e $d_{g}(., q)^{-1}\left(\left(0, d_{g}(p, q)\right)\right)$. Entretanto, como sabemos (Proposição 2.4) que $d_{g}$ é duas vezes diferenciável em $d_{g}(p, .)^{-1}\left(\left(0, d_{g}(p, q)\right)\right)$ e $d_{g}(., q)^{-1}\left(\left(0, d_{g}(p, q)\right)\right)$ a menos de um conjunto $\mathscr{U}$ satisfazendo $\mu_{g}(\mathscr{U})=\mu_{d_{g}}(\mathscr{U})=0$, todas as fórmulas que obtivemos continuam valendo nos pontos onde $d_{g}(p, .)^{2}$ e $d_{g}(., q)^{2}$ são duas vezes diferenciáveis. Não podemos, todavia, garantir a validade dos limites de expressões envolvendo segundas derivadas de $d_{g}(., q)^{2}$ à medida que $r \rightarrow p$, ou envolvendo segundas derivadas de $d_{g}(p, .)^{2}$ à medida que $r \rightarrow q$, uma vez que nada sabemos sobre a continuidade destas fora de vizinhanças geodesicamente convexas.

2. O segundo problema é específico de espaços-tempos (localmente) AAdS. Pode ocorrer que (um)a geodésica tipo tempo maximal (com respeito a $d_{\bar{g}}$ ) ligando $p$ a $q$ passe por $\mathscr{M}$ ao invés de permanecer em $\mathscr{I}$. Isso implica não só que $T^{a}$ deixa de ser tangente a $\mathscr{I}$, como também faz com que $T^{a}$ possua pontos de descontinuidade em $\mathscr{I}$. A hipótese de que tal focalização de geodésicas tipo tempo não ocorre é mais forte e não pode ser obtida como conseqüência de que toda geodésica nula em $\overline{\mathscr{M}}$ com extremos em $\mathscr{I}$ e atravessando $\mathscr{M}$ tem um par de pontos conjugados, empregada por exemplo no Teorema 1.3. Podemos, é claro, impor a hipótese de que geodésicas causais maximais em $\overline{\mathscr{M}}$ ligando pontos $p$ e $q$ necessariamente pertencem a $\mathscr{I}$, em linhas similares ao que fizemos com geodésicas nulas ao longo do Capítulo 1. Entretanto, essa hipótese tem a característica inconveniente de depender globalmente do fator conforme $z$, ao contrário do caso mais fraco de geodésicas nulas, que só depende da estrutura causal. 
Uma maneira de contornar esses problemas é regularizar $\lambda_{p, q}^{g}$ de modo que a regularização, denotada aqui $\bar{\lambda}_{p, q}^{g}$ por concreteza, satisfaça $\bar{\lambda}_{p, q}^{g} \Upsilon_{\mathscr{D}_{p, q}}=\lambda$, onde $u_{p, q}^{\lambda}$ é dado por (1.26). O problema aqui é como regularizar uma função tempo global definida num espaço-tempo com bordo - resolveremos o problema da seguinte forma: definamos o duplo ${ }^{2} \mathscr{M}$ de um espaço-tempo localmente tipo AdS $\overline{\mathscr{M}}$ como a união disjunta de duas cópias de $\overline{\mathscr{M}}$ com os pontos em $\mathscr{I}$ identificados. ${ }^{2} \overline{\mathscr{M}}$ pode ser imbuído de uma estrutura diferenciável compatível com a de cada cópia e tal que a inclusão natural de cada uma das cópias é um mergulho diferenciável [Hir76]. A métrica em ${ }^{2} \overline{\mathscr{M}}$ induzida por $\bar{g}$ (também denotada $\bar{g}$, uma vez que não há confusão) é $\mathscr{C}^{\infty}$ no interior de cada cópia, e $\mathscr{C}^{d-1,1}\left(\operatorname{resp} . \mathscr{C}^{\infty}\right)$ em $\mathscr{I}$ para $d$ ímpar (resp. $d$ par ${ }^{3}$ ), devido à expansão de FEFFERMAN-GRAHAM para espaços-tempos localmente tipo AdS construída na Seção anterior - a regularidade é $\mathscr{C}^{\infty}$ para $d$ arbitrário se $(\mathscr{M}, g)$ é localmente AAdS ou, mais em geral, se $\bar{g}^{(0)}$ possui um representante $\bar{g}^{(0)^{\prime}}$ na sua classe conforme que satisfaça $\operatorname{Ric}\left(\bar{g}^{(0)^{\prime}}\right)=0$. Esta construção é uma generalização da identificação conforme de $A d S_{d}$ com metade de $E S U_{d}$, vista no Capítulo 1, página 10.

Podemos estender $z$ a $z<0$ em ${ }^{2} \overline{\mathscr{M}}$, definindo assim um sistema de coordenadas Gaussianas normais ao redor de $\mathscr{I}$. O duplo $\left({ }^{2} \overline{\mathscr{M}}, \bar{g}\right)$ possui um grupo de isometrias globais, isomorfo a $\mathbb{Z}_{2}$, gerado pela reflexão $\zeta$ através de $\mathscr{I}$ de um ponto numa cópia de $\mathscr{M}$, tendo como imagem sua contraparte na outra cópia (localmente perto de $\mathscr{I}$, temos $z \circ \zeta=-z)$.

Definiremos agora $\bar{\lambda}_{p, q}^{\bar{g}}$ num espaço-tempo AAdS causalmente simples $(\mathscr{M}, g)$ que satisfaz a hipótese de focalização do Teorema 1.3, página 17 (esses requerimentos garantem, como vimos no Capítulo 1 , que $\left.I^{+}(p, \overline{\mathscr{M}}) \cap I^{-}(q, \overline{\mathscr{M}}) \cap \mathscr{I}=\mathscr{D}_{p, q}\right)$, seguindo os seguintes passos:

- Primeiro, definimos $\lambda_{p, q}^{\bar{g}}$ em $\left({ }^{2} \overline{\mathscr{M}}, \bar{g}\right)$ (mais precisamente, em $\mathscr{O}_{p, q}=\mathscr{W}_{p, q} \cup \mathscr{D}_{p, q} \cup$ $\left.\zeta\left(\mathscr{W}_{p, q}\right)\right)$, com a mesma fórmula.

- Em seguida, regularizamos $\lambda_{p, q}^{\bar{g}}$ empregando o método de BERnAl e SÁnchez [BS03, BS05] (ver [Sán05] para um esboço introdutório do método), obtendo uma função tempo global $\mathscr{C}^{\infty} \tilde{\lambda}_{p, q}^{\bar{g}}$ que folheia $\mathscr{O}_{p, q}$ por superfícies de CAUCHY. Assumimos ainda que a regularização obtida satisfaz $\left|\tilde{\lambda}_{p, q}^{\bar{g}}-\lambda_{p, q}^{\bar{g}}\right|<\delta$ para $\delta<1$ suficientemente pequeno [Sei77].

- Definamos $\hat{\lambda}_{p, q}^{\bar{g}}=\frac{1}{2}\left(\tilde{\lambda}_{p, q}^{\bar{g}}+\tilde{\lambda}_{p, q}^{\bar{g}} \circ \zeta\right)$. Como tanto $\tilde{\lambda}_{p, q}^{\bar{g}}$ como $\tilde{\lambda}_{p, q}^{\bar{g}} \circ \zeta$ são funções tempo globais em $\mathscr{O}_{p, q}$ cujas hipersuperfícies de nível são superfícies de CAUCHY em $\mathscr{O}_{p, q}$,

\footnotetext{
${ }^{3} \mathrm{~A}$ regularidade adicional deve-se (i) à ausência de termos logarítmicos na expansão de FefFerman-Graham e (ii) à simetria de paridade em $z$ na fórmula (2.10), página 35, que dá origem às relações de recursão.
} 
e a coleção de tais funções é fechada por combinações lineares convexas [Sei77], segue que $\hat{\lambda}_{p, q}^{\bar{g}}$ também satisfaz essas propriedades, mais a propriedade adicional (que será crucial em breve) $\partial_{z} \hat{\lambda}_{p, q}^{\bar{g}}\lceil\mathscr{I}=0$ e a estimativa no item anterior. A partir daqui, a utilidade do duplo acaba - restringimos $\hat{\lambda}_{p, q}^{\bar{g}}$ a $\overline{\mathscr{M}}$, e prosseguimos apenas nesta variedade.

- Estendemos $\lambda_{p, q}^{\bar{g}^{(0)}}$ de $\mathscr{D}_{p, q}$ a uma vizinhança de colarinho $\mathscr{U}_{p, q}^{\delta^{\prime}} \cong \mathscr{D}_{p, q} \times\left[0, \delta^{\prime}>\right.$ $0) \ni(x, w)$ deste em $\mathscr{W}_{p, q}$ pela fórmula $\lambda_{p, q}^{\bar{g}^{(0)}}(x, w)=\lambda_{p, q}^{\bar{g}^{(0)}}(x)$, tomando $\delta^{\prime}$ pequeno o suficiente para que $\bar{\nabla}^{a} \lambda^{\bar{g}^{(0)}}$ permaneça tipo tempo em $\mathscr{U}_{p, q}^{\delta^{\prime}}$ e mantendo a mesma notação para a extensão (note que tal restrição a $\delta^{\prime}$ é independente da restrição a $\delta$ no primeiro item).

- Escolhemos uma partição de unidade $\{\phi, 1-\phi\}$ de $\mathscr{W}_{p, q} \cup \mathscr{D}_{p, q}$ subordinada ao recobrimento $\left\{\mathscr{D}_{p, q} \times\left[0, \frac{3 \delta^{\prime}}{4}\right), \mathscr{W}_{p, q} \backslash\left(\mathscr{D}_{p, q} \times\left[0, \frac{\delta^{\prime}}{2}\right]\right\}\right.$, satisfazendo $\left|\partial_{w} \phi\right| \leq K\left(\delta^{\prime}\right)^{-1}$ [Whi34] e independente de $x \in \mathscr{D}_{p, q}$ (como $\phi \equiv 0$ fora de $\mathscr{U}_{p, q}^{\delta^{\prime}}$, não há problemas com a segunda propriedade). Definamos

$$
\bar{\lambda}_{p, q}^{\bar{g}} \doteq \phi \lambda_{p, q}^{\bar{g}^{(0)}}+(1-\phi) \hat{\lambda}_{p, q}^{\bar{g}},
$$

que satisfaz

$$
d \bar{\lambda}_{p, q}^{\bar{g}}=\left(\lambda_{p, q}^{\bar{g}^{(0)}}-\hat{\lambda}_{p, q}^{\bar{g}}\right) \partial_{w} \phi d w+\phi d \lambda_{p, q}^{\bar{g}^{(0)}}+(1-\phi) d \hat{\lambda}_{p, q}^{\bar{g}}
$$

- Vemos imediatamente que precisamos controlar o tamanho de $\left|\lambda_{p, q}^{\bar{g}^{(0)}}-\hat{\lambda}_{p, q}^{\bar{g}}\right|$ em $\mathscr{U}_{p, q}^{\delta^{\prime}}$ tanto para que $\left|\bar{\lambda}_{p, q}^{\bar{g}^{(0)}}-\hat{\lambda}_{p, q}^{\bar{g}}\right|$ seja tão pequeno quanto queiramos e, ao mesmo tempo, $d \bar{\lambda}_{p, q}^{\bar{g}^{(0)}}$ seja um campo de covetores tipo tempo futuros; o impedimento mais severo a tais requerimentos reside no covetor tipo espaço proporcional a $\partial_{w} \phi$ em (2.46), devido à estimativa de Whitney invocada no item anterior, que é essencialmente optimal. Escrevemos, pois,

$$
\left|\lambda_{p, q}^{\bar{g}^{(0)}}(x, w)-\hat{\lambda}_{p, q}^{\bar{g}}(x, w)\right| \leq \underbrace{\left|\lambda_{p, q}^{\bar{g}^{(0)}}(x)-\hat{\lambda}_{p, q}^{\bar{g}}(x, 0)\right|}_{<\delta(a)}+\underbrace{\left|\hat{\lambda}_{p, q}^{\bar{g}}(x, w)-\hat{\lambda}_{p, q}^{\bar{g}}(x, 0)\right|}_{\leq K^{\prime} \delta^{\prime 2}(b)}
$$

onde a estimativa ( $a$ ) é válida por construção, e a estimativa $(b)$ decorre do fato de que $\hat{\lambda}_{p, q}^{\bar{g}}$ é $\mathscr{C}^{\infty}$ e $\partial_{w} \hat{\lambda}_{p, q}^{\bar{g}}\left\lceil_{\mathscr{D}_{p, q}} \equiv 0\right.$.

- Tomando $\delta=K^{\prime}\left(\delta^{\prime}\right)^{2}$ e $\delta^{\prime}$ suficientemente pequeno, segue que $\bar{\lambda}_{p, q}^{\bar{g}}$ é uma função tempo global $\mathscr{C}^{\infty}$ que:

1. satisfaz $\left|\bar{\lambda}_{p, q}^{\bar{g}}-\lambda_{p, q}^{\bar{g}}\right|<\delta^{\prime \prime}$, onde $\delta^{\prime \prime}>0$ é tão pequeno quanto se queira,

2. estende $\lambda_{p, q}^{\bar{g}^{(0)}}$ a $\mathscr{W}_{p, q} \cup \mathscr{D}_{p, q}$, e tal que 
3. $\bar{\nabla}^{a} \bar{\lambda}_{p, q}^{\bar{g}}$ é um campo vetorial tipo tempo futuro e tangente a $\mathscr{I}$, e

4. $\left(\bar{\lambda}_{p, q}^{\bar{g}}\right)^{-1}(t)$ é uma superfície de CAUCHY para $\mathscr{W}_{p, q} \cup \mathscr{D}_{p, q}$ para todo $t \in \mathbb{R}$ (segue diretamente da primeira propriedade [Die88] e da continuidade de $\left.\bar{\lambda}_{p, q}^{\bar{g}}\right)$.

- Em virtude dos resultados acima, segue que

$$
\bar{T}^{a} \doteq \frac{1}{\bar{g}^{-1}\left(d \bar{\lambda}_{p, q}^{\bar{g}}, d \bar{\lambda}_{p, q}^{\bar{g}}\right)} \bar{\nabla}^{a} \bar{\lambda}_{p, q}^{\bar{g}}
$$

é um campo de KILling assintótico em $\left(\mathscr{W}_{p, q}, g\left\lceil_{\mathscr{W}_{p, q}}\right)\right.$ que satisfaz $\bar{T}^{a} \uparrow_{\mathscr{I}}=\frac{d u_{p, q}}{d \lambda}$, e, portanto, as fórmulas (2.43) e (2.44), como desejávamos.

Apesar de $\bar{\lambda}_{p, q}^{\bar{g}}$ ter todas as propriedades de que necessitamos, ela infelizmente não possui mais em $(\mathscr{M}, g)$ a interpretação geométrica natural que $\lambda_{p, q}^{\bar{g}}$ possui, embora esteja tão próxima disto quanto queiramos. Não obstante, mostramos que é possível construir explicitamente famílias de isometrias assintóticas em (praticamente) qualquer cunha. Faremos uso extensivo deste fato no Capítulo 4.

\subsection{3 "Retorno ao equilíbrio" em cunhas}

No trabalho de MARTinetti e Rovelli [MR03], foi proposto associar um "efeito UnRUH" ao grupo $u_{p, q}^{\lambda}$ associado a um diamante $\mathscr{D}_{p, q}$ no espaço-tempo de MinkowsKi, cujas órbitas foram imaginadas como as linhas-mundo de "observadores com tempo de vida finito". Para teorias quânticas de campo com vácuo conformalmente invariante, é realmente possível provar tal associação [BGL93], mas uma discussão apropriada deste ponto terá de aguardar o Capítulo 4. Não obstante, as fórmulas (2.43) e (2.44) tornam natural a seguinte pergunta: e se tal temperatura fosse atingida somente no limite $\lambda \rightarrow \pm \infty$ ? Em teorias quânticas de campo dotadas de liberdade assintótica, invariância de escala de fato é realizada somente no limite de curtas distâncias, sendo as correções ao comportamento "crítico" dadas, por exemplo, pela expansão de produtos de operadores (OPE). Assim, podemos pensar que a "Hipótese de Tempo Térmico" de Martinetti e Rovelli, citada acima, só é realizada na forma mais fraca de um retorno ao equilíbrio, em razão de uma invariância assintótica de escala (daremos um sentido mais preciso a estas considerações no Capítulo 4).

Transplantemos este raciocínio para cunhas AAdS. Do ponto de vista das leis da dinâmica de buracos negros, se imaginarmos a fronteira de uma cunha (suposta geodesicamente convexa, por simplicidade) $\mathscr{W}_{p, q}$ como um horizonte de eventos "assintoticamente estacionário", e $\mathscr{W}_{p, q}$ como um "domínio de comunicação exterior", podemos nos perguntar se existe algum análogo assintótico para essas leis. É agora nosso objetivo 
mostrar que isso de fato ocorre, pelo menos para a lei zero.

Primeiramente, vamos dar a definição precisa de gravidade de superfície no nosso contexto. Seja $T^{a}$ como em (2.42). Tomando, por exemplo, $r \rightarrow r_{-} \in \partial_{-} \mathscr{W}_{p, q}$, temos que $\Gamma_{\bar{g}}\left(p, r_{-}\right)=0, \Gamma_{\bar{g}}\left(r_{-}, q\right) \neq 0$ e $d \Gamma_{\bar{g}}\left(p, r_{-}\right) \neq 0$. Mais ainda,

$$
\lim _{r \rightarrow r_{-}} \bar{g}(T, T)=-\lim _{r \rightarrow r_{-}} \frac{2 \Gamma_{\bar{g}}(p, r) \Gamma_{\bar{g}}(r, q)}{\Gamma_{\bar{g}}(p, r)+\Gamma_{\bar{g}}(r, q)+\bar{g}^{-1}\left(d \Gamma_{\bar{g}}(p, r), d \Gamma_{\bar{g}}(r, q)\right)}=0
$$

por construção. Igualmente, se $r \rightarrow r_{+} \in \partial_{+} \mathscr{W}_{p, q}$, temos que $\Gamma_{\bar{g}}\left(r_{+}, q\right)=0, \Gamma_{\bar{g}}\left(p, r_{+}\right) \neq$ $0, d \Gamma_{\bar{g}}\left(r_{+}, q\right) \neq 0$ e $\lim _{r \rightarrow r_{+}} \bar{g}(T, T)=0$. No caso em que $r \rightarrow r_{0} \in \partial I^{+}(p, \overline{\mathscr{M}}) \cap$ $\partial I^{-}(q, \overline{\mathscr{M}})$, temos que $T^{a} \rightarrow 0$. Em suma, $T^{a}$ é, ao mesmo tempo, tangente e normal ao horizonte passado $\partial_{-} \mathscr{W}_{p, q}$ e ao horizonte futuro $\partial_{+} \mathscr{W}_{p, q}$ de $\mathscr{W}_{p, q}$ (notar que a definição de horizonte que demos na página 15 exclui precisamente os pontos de $\partial \mathscr{W}_{p, q}$ onde $\left.T^{a} \rightarrow 0\right)$. Assim, temos que

$$
\lim _{\rightarrow \partial_{ \pm} \mathscr{W}_{p, q}} \bar{\nabla}_{a} \bar{g}(T, T) \doteq-2 \bar{\kappa}_{ \pm}\left(\lim _{\rightarrow \partial_{ \pm} \mathscr{W}_{p, q}} T_{a}\right),
$$

onde $\bar{\kappa}_{+}\left(\right.$resp. $\left.\bar{\kappa}_{-}\right)$é uma função $\mathscr{C}^{\infty}$ na subvariedade $\partial_{+} \mathscr{W}_{p, q}\left(\right.$ resp. $\left.\partial_{-} \mathscr{W}_{p, q}\right)$, dada por

$$
\bar{\kappa}_{ \pm}=-\lim _{\rightarrow \partial_{ \pm} \mathscr{W}_{p, q}} \frac{T^{a} \bar{\nabla}_{a} \bar{g}(T, T)}{2 \bar{g}(T, T)}=\frac{g^{-1}\left(d \lambda_{p, q}^{\bar{g}}, d \bar{g}^{-1}\left(d \lambda_{p, q}^{\bar{g}}, d \lambda_{p, q}^{\bar{g}}\right)\right)}{2 \bar{g}^{-1}\left(d \lambda_{p, q}^{\bar{g}}, d \lambda_{p, q}^{\bar{g}}\right)^{2}} .
$$

Suponhamos momentaneamente que $T^{a}$ é um campo de KILLING conforme com respeito a $\bar{g}$. Neste caso,

$$
\begin{aligned}
\bar{\nabla}_{a} \bar{g}(T, T) & =2 T^{b} \bar{\nabla}_{a} T_{b}=2 T^{b}\left(\bar{\nabla}_{a} T_{b}+\bar{\nabla}_{b} T_{a}\right)-2 T^{b} \bar{\nabla}_{b} T_{a}= \\
& =\frac{4}{d}\left(\bar{\nabla}_{b} T^{b}\right) T_{a}-2 T^{b} \bar{\nabla}_{b} T_{a} \stackrel{\rightarrow \partial_{ \pm} \mathscr{O}_{p, q}}{\longrightarrow}-2 \bar{\kappa}_{ \pm} T_{a},
\end{aligned}
$$

de onde segue que

$$
\kappa_{ \pm} \doteq \lim _{\rightarrow \partial_{ \pm} \mathscr{W}_{p, q}} \frac{T^{a} T^{b} \bar{\nabla}_{b} T_{a}}{\bar{g}(T, T)} \doteq \lim _{\rightarrow \partial_{ \pm} \mathscr{W}_{p, q}} \frac{T^{a} T^{b} \bar{\nabla}_{a} T_{b}}{\bar{g}(T, T)}+\bar{\kappa}_{ \pm}=\lim _{\rightarrow \partial_{ \pm} \mathscr{W}_{p, q}}\left(\frac{2}{d} \bar{\nabla}_{a} T^{a}\right)+\bar{\kappa}_{ \pm}
$$

define precisamente a falha da parametrização das órbitas de $T^{a}$ em $\partial_{ \pm} \mathscr{D}_{p, q}$ em ser uma parametrização afim, uma vez que $T^{a}$ é tangente às geodésicas nulas que geram $\partial_{+} \mathscr{W}_{p, q}$ e $\partial_{-} \mathscr{W}_{p, q}$. Mais precisamente, se $\lambda_{-}$(resp. $\left.\lambda_{+}\right)$é um parâmetro afim comum às geodésicas nulas futuras que emanam de $p$ (resp. atingem $q$ ) tal que $\lambda_{-}=0$ em $p$ (resp. $\lambda_{+}=0$ em $q$ ), temos que

$$
\lambda_{ \pm}=\mp e^{\kappa_{ \pm} \lambda_{p, q}^{g}}
$$

Outra interpretação para $\kappa_{ \pm}$, que segue das fórmulas (2.51)-(2.53), é a aceleração necessária para manter um corpo de teste a uma distância fixa, mas "infinitesimalmente pequena", do horizonte, compensada pelo fator de "redshift" $(-\bar{g}(T, T))^{\frac{1}{2}}$ da órbita [Wal84, Wal94]. 
DEFINIÇÃO $2.2 \kappa_{-}$(resp. $\left.\kappa_{+}\right)$é dita ser a gravidade de superfície passada (resp. futura) $d e \mathscr{O}_{p, q}$.

Para $T^{a}$ arbitrário, usamos a(s duas) primeira(s) igualdade(s) em (2.53) para definir $\kappa_{ \pm}$, mas a última igualdade na fórmula $(2.53)$ é só assintoticamente válida, à medida que $r_{-} \rightarrow p, r_{+} \rightarrow q$. Com essa ressalva em mente, podemos agora formular nossa "lei zero da dinâmica":

Teorema 2.6 (Lei Zero da Dinâmica de Cunhas AAdS) Seja $(\mathscr{M}, g)$ um espaçotempo (localmente) AAdS, e $\mathscr{W}_{p, q}$ uma cunha contida numa vizinhança geodesicamente convexa em $(\overline{\mathscr{M}}, g)$. Então $\lim _{\rightarrow p} \kappa_{-}=-\lim _{\rightarrow q} \kappa_{+}=1$.

Prova. Um cálculo direto empregando as propriedades de $\Gamma_{\bar{g}}$ nos dá

$$
\begin{gathered}
T^{a} \bar{\nabla}_{a} \bar{g}(T, T)=\frac{2 \Gamma_{\bar{g}}(p, r) \Gamma_{\bar{g}}(r, q)}{\left(\Gamma_{\bar{g}}(p, r)+\Gamma_{\bar{g}}(r, q)+\bar{g}^{-1}\left(d \Gamma_{\bar{g}}(p, r), d \Gamma_{\bar{g}}(r, q)\right)\right)^{2}} . \\
\cdot\left[\left(\Gamma_{\bar{g}}(r, q)-\Gamma_{\bar{g}}(p, r)\right)\left(2+\frac{\bar{g}^{-1}\left(d \Gamma_{\bar{g}}(p, r), d \Gamma_{\bar{g}}(r, q)\right)}{\Gamma_{\bar{g}}(p, r)+\Gamma_{\bar{g}}(r, q)+\bar{g}^{-1}\left(d \Gamma_{\bar{g}}(p, r), d \Gamma_{\bar{g}}(r, q)\right)}\right)+\right. \\
\left.-\frac{\Gamma_{\bar{g}}(r, q) \bar{\nabla}^{a} \Gamma_{\bar{g}}(p, r) \bar{\nabla}^{b} \Gamma_{\bar{g}}(p, r) \bar{\nabla}_{a} \bar{\nabla}_{b} \Gamma_{\bar{g}}(r, q)-\Gamma_{\bar{g}}(p, r) \bar{\nabla}^{a} \Gamma_{\bar{g}}(r, q) \bar{\nabla}^{b} \Gamma_{\bar{g}}(r, q) \bar{\nabla}_{a} \bar{\nabla}_{b} \Gamma_{\bar{g}}(p, r)}{\Gamma_{\bar{g}}(p, r)+\Gamma_{\bar{g}}(r, q)+\bar{g}^{-1}\left(d \Gamma_{\bar{g}}(p, r), d \Gamma_{\bar{g}}(r, q)\right)}\right],
\end{gathered}
$$

e, portanto,

$$
\begin{gathered}
-\frac{T^{a} \bar{\nabla}_{a} \bar{g}(T, T)}{2 \bar{g}(T, T)}=-\frac{1}{2\left(\Gamma_{\bar{g}}(p, r)+\Gamma_{\bar{g}}(r, q)+\bar{g}^{-1}\left(d \Gamma_{\bar{g}}(p, r), d \Gamma_{\bar{g}}(r, q)\right)\right)} . \\
.\left[\left(\Gamma_{\bar{g}}(r, q)-\Gamma_{\bar{g}}(p, r)\right)\left(2+\frac{\bar{g}^{-1}\left(d \Gamma_{\bar{g}}(p, r), d \Gamma_{\bar{g}}(r, q)\right)}{\Gamma_{\bar{g}}(p, r)+\Gamma_{\bar{g}}(r, q)+\bar{g}^{-1}\left(d \Gamma_{\bar{g}}(p, r), d \Gamma_{\bar{g}}(r, q)\right)}\right)+\right. \\
\left.-\frac{\Gamma_{\bar{g}}(r, q) \bar{\nabla}^{a} \Gamma_{\bar{g}}(p, r) \bar{\nabla}^{b} \Gamma_{\bar{g}}(p, r) \bar{\nabla}_{a} \bar{\nabla}_{b} \Gamma_{\bar{g}}(r, q)-\Gamma_{\bar{g}}(p, r) \bar{\nabla}^{a} \Gamma_{\bar{g}}(r, q) \bar{\nabla}^{b} \Gamma_{\bar{g}}(r, q) \bar{\nabla}_{a} \bar{\nabla}_{b} \Gamma_{\bar{g}}(p, r)}{\Gamma_{\bar{g}}(p, r)+\Gamma_{\bar{g}}(r, q)+\bar{g}^{-1}\left(d \Gamma_{\bar{g}}(p, r), d \Gamma_{\bar{g}}(r, q)\right)}\right] .
\end{gathered}
$$

$\bar{\kappa}_{+}\left(\right.$resp. $\left.\bar{\kappa}_{-}\right)$é obtida tomando-se o limite $\Gamma_{\bar{g}}(r, q) \searrow 0, d \Gamma_{\bar{g}}(r, q) \neq 0$ (resp.

$\left.\Gamma_{\bar{g}}(p, r) \searrow 0, d \Gamma_{\bar{g}}(p, r) \neq 0\right)$. Assim, temos que

$$
\begin{gathered}
\bar{\kappa}_{+}=\frac{1}{2\left(\Gamma_{\bar{g}}(p, r)+\bar{g}^{-1}\left(d \Gamma_{\bar{g}}(p, r), d \Gamma_{\bar{g}}(r, q)\right)\right)} . \\
\cdot\left[\Gamma_{\bar{g}}(p, r)\left(3-\frac{\Gamma_{\bar{g}}(p, r)}{\Gamma_{\bar{g}}(p, r)+\bar{g}^{-1}\left(d \Gamma_{\bar{g}}(p, r), d \Gamma_{\bar{g}}(r, q)\right)}\right)+\right. \\
\left.-\frac{\Gamma_{\bar{g}}(p, r) \bar{\nabla}^{a} \Gamma_{\bar{g}}(r, q) \bar{\nabla}^{b} \Gamma_{\bar{g}}(r, q) \bar{\nabla}_{a} \bar{\nabla}_{b} \Gamma_{\bar{g}}(p, r)}{\Gamma_{\bar{g}}(p, r)+\bar{g}^{-1}\left(d \Gamma_{\bar{g}}(p, r), d \Gamma_{\bar{g}}(r, q)\right)}\right]
\end{gathered}
$$

$\mathrm{e}$

$$
\bar{\kappa}_{-}=-\frac{1}{2\left(\Gamma_{\bar{g}}(r, q)+\bar{g}^{-1}\left(d \Gamma_{\bar{g}}(p, r), d \Gamma_{\bar{g}}(r, q)\right)\right)} .
$$




$$
\begin{gathered}
\cdot\left[\Gamma_{\bar{g}}(r, q)\left(3-\frac{\Gamma_{\bar{g}}(r, q)}{\Gamma_{\bar{g}}(r, q)+\bar{g}^{-1}\left(d \Gamma_{\bar{g}}(p, r), d \Gamma_{\bar{g}}(r, q)\right)}\right)+\right. \\
\left.-\frac{\Gamma_{\bar{g}}(r, q) \bar{\nabla}^{a} \Gamma_{\bar{g}}(p, r) \bar{\nabla}^{b} \Gamma_{\bar{g}}(p, r) \bar{\nabla}_{a} \bar{\nabla}_{b} \Gamma_{\bar{g}}(r, q)}{\Gamma_{\bar{g}}(r, q)+\bar{g}^{-1}\left(d \Gamma_{\bar{g}}(p, r), d \Gamma_{\bar{g}}(r, q)\right)}\right]
\end{gathered}
$$

A divergência de $T^{a}$, por sua vez, dá

$$
\begin{gathered}
\bar{\nabla}_{a} T^{a}=\frac{1}{\Gamma_{\bar{g}}(p, r)+\Gamma_{\bar{g}}(r, q)+\bar{g}^{-1}\left(d \Gamma_{\bar{g}}(p, r), d \Gamma_{\bar{g}}(r, q)\right)} . \\
{\left[\Gamma_{\bar{g}}(p, r) \bar{\nabla}_{a} \bar{\nabla}^{a} \Gamma_{\bar{g}}(r, q)-\Gamma_{\bar{g}}(r, q) \bar{\nabla}_{a} \bar{\nabla}^{a} \Gamma_{\bar{g}}(p, r)-\frac{1}{\Gamma_{\bar{g}}(p, r)+\Gamma_{\bar{g}}(r, q)+\bar{g}^{-1}\left(d \Gamma_{\bar{g}}(p, r), d \Gamma_{\bar{g}}(r, q)\right)} \cdot\right.} \\
\cdot\left(\left(\Gamma_{\bar{g}}(p, r)-\Gamma_{\bar{g}}(r, q)\right) \bar{g}^{-1}\left(d \Gamma_{\bar{g}}(p, r), d \Gamma_{\bar{g}}(r, q)\right)+\Gamma_{\bar{g}}(r, q) \bar{\nabla}^{a} \Gamma_{\bar{g}}(p, r) \bar{\nabla}^{b} \Gamma_{\bar{g}}(p, r) \bar{\nabla}_{a} \bar{\nabla}_{b} \Gamma_{\bar{g}}(r, q)+\right. \\
\left.\left.-\Gamma_{\bar{g}}(p, r) \bar{\nabla}^{a} \Gamma_{\bar{g}}(r, q) \bar{\nabla}^{b} \Gamma_{\bar{g}}(r, q) \bar{\nabla}_{a} \bar{\nabla}_{b} \Gamma_{\bar{g}}(p, r)\right)\right],
\end{gathered}
$$

que no horizonte futuro $\partial_{+} \mathscr{W}_{p, q}$ fica

$$
\begin{gathered}
\left.\bar{\nabla}_{a} T^{a}\right|_{\partial_{+} \mathscr{W}_{p, q}}=\frac{1}{\Gamma_{\bar{g}}(p, r)+\bar{g}^{-1}\left(d \Gamma_{\bar{g}}(p, r), d \Gamma_{\bar{g}}(r, q)\right)} . \\
{\left[\Gamma_{\bar{g}}(p, r) \bar{\nabla}_{a} \bar{\nabla}^{a} \Gamma_{\bar{g}}(r, q)-\frac{1}{\Gamma_{\bar{g}}(p, r)+\bar{g}^{-1}\left(d \Gamma_{\bar{g}}(p, r), d \Gamma_{\bar{g}}(r, q)\right)} \cdot\right.} \\
\left.\cdot\left(\Gamma_{\bar{g}}(p, r) \bar{g}^{-1}\left(d \Gamma_{\bar{g}}(p, r), d \Gamma_{\bar{g}}(r, q)\right)-\Gamma_{\bar{g}}(p, r) \bar{\nabla}^{a} \Gamma_{\bar{g}}(r, q) \bar{\nabla}^{b} \Gamma_{\bar{g}}(r, q) \bar{\nabla}_{a} \bar{\nabla}_{b} \Gamma_{\bar{g}}(p, r)\right)\right], \\
\text { e no horizonte passado } \partial_{-} \mathscr{W}_{p, q}, \\
\left.\quad \bar{\nabla}_{a} T^{a}\right|_{\partial_{-} \mathscr{W}_{p, q}}=-\frac{1}{\Gamma_{\bar{g}}(r, q)+\bar{g}^{-1}\left(d \Gamma_{\bar{g}}(p, r), d \Gamma_{\bar{g}}(r, q)\right)} . \\
\cdot\left(\Gamma_{\bar{g}}(r, q) \bar{\nabla}_{a} \bar{\nabla}^{a} \Gamma_{\bar{g}}(p, r)-\frac{1}{\Gamma_{\bar{g}}(r, q)+\bar{g}^{-1}\left(d \Gamma_{\bar{g}}(p, r), d \Gamma_{\bar{g}}(r, q)\right)} .\right.
\end{gathered}
$$

Obtemos imediatamente das fórmulas acima que

$$
\lim _{\rightarrow q} \bar{\kappa}_{+}=1=-\lim _{\rightarrow p} \bar{\kappa}_{-}, \lim _{\rightarrow q} \bar{\nabla}_{a} T^{a}=-d=-\lim _{\rightarrow p} \bar{\nabla}_{a} T^{a} .
$$

O resultado segue então da fórmula (2.53) e da discussão que segue a Definição 2.2 .

OBSERVAÇÃO 2.3 O resultado e a demonstração do Teorema 2.6 valem para diamantes geodesicamente convexos $\mathscr{O}_{p, q}$ em espaços-tempos causalmente simples arbitrários sem nenhuma alteração. 
Há duas ressalvas que devem ser feitas acerca do Teorema 2.6 no que concerne a interpretação da cunha $\mathscr{W}_{p, q}$ como o exterior de um horizonte de eventos.

- Todos os resultados desta Subseção envolvem a métrica $\bar{g}$ do fecho conforme, e não a métrica física $g$. Temos, no entanto, que

$$
\frac{T^{a} \bar{\nabla}_{a} \bar{g}(T, T)}{\bar{g}(T, T)}=\frac{T^{a} \nabla_{a} g(T, T)}{g(T, T)}+2 z\langle d z, T\rangle
$$

e

$$
\begin{aligned}
\bar{\nabla}_{a} T^{a} & =\bar{g}^{a b} \bar{\nabla}_{a} T_{b}=\frac{1}{z^{2}} g^{a b} \bar{\nabla}_{a} T_{b}= \\
& =\frac{1}{z^{2}} \nabla_{a} T^{a}-\frac{1}{z^{2}} g^{a b} C_{a b}^{c} T_{c}=\frac{1}{z^{2}} \nabla_{a} T^{a}+\frac{d-2}{2 z^{3}}\langle d z, T\rangle \Rightarrow \\
& \Rightarrow \nabla_{a} T^{a}=z^{2} \bar{\nabla}_{a} T^{a}-\frac{d-2}{2 z}\langle d z, T\rangle,
\end{aligned}
$$

onde $C_{a b}^{c}$ é o tensor (A.27) que relaciona as conexões $\bar{\nabla}$ e $\nabla$ (ver página 120). Como $T^{a}$ é tangente a $\mathscr{I}$ e se anula em $p$ e $q$ (ver fórmula (2.44), página 47), segue que $\langle d z, T\rangle=o(z)$ à medida que nos aproximamos de $p$ ou $q$. Levando em consideração que $T^{a}$ é um campo de KILLING assintótico, obtemos que $\kappa_{ \pm}$define também a gravidade de superfície assintótica passada/futura com respeito a $g$ à medida que nos aproximamos de $p, q$. Como os valores assintóticos para $\kappa_{ \pm}$são os mesmos para todo campo de KILling assintótico a $\frac{d u_{p, q}^{\lambda}}{d \lambda}$ em $\mathscr{W}_{p, q}$ à medida que $z \searrow 0$, segue que a conclusão do Teorema 2.6 vale também para cunhas não geodesicamente convexas e os campos de KILLING assintóticos construídos na Subseção 2.3.2.

- O sinal de $\kappa_{+}$que obtivemos é o contrário do que se espera de um horizonte de eventos, pois a origem do nosso parâmetro afim para os geradores (orientados para o futuro) de $\partial_{+} \mathscr{W}_{p, q}$ corresponde a $q$ e não a $\partial I^{+}(p, \overline{\mathscr{M}}) \cap \partial I^{-}(q, \overline{\mathscr{M}})$, que corresponderia à "superfície de bifurcação" do horizonte (note a mudança de sinal em (2.54)). Uma correção deste problema leva à mudança desejada do sinal de $\kappa_{+}$.

Notemos, por fim, que há uma hipótese sobre a normalização de $T^{a}$ implícita na definição de $\kappa_{ \pm}$. Mais precisamente, nossa definição está condicionada ao seguinte fato: se $\Gamma_{\bar{g}^{(0)}}(p, r)=\Gamma_{\bar{g}^{(0)}}(r, q)$, i.e., $r$ é o ponto médio da geodésica tipo tempo maximal em $\mathscr{M}$ in $(r) \ni p, q$ ligando $p$ e $q$, implicando claramente que ${ }^{(0)} \bar{\nabla}^{a} \Gamma_{\bar{g}^{(0)}}(p, r)=$ ${ }^{(0)} \bar{\nabla}^{a} \Gamma_{\bar{g}^{(0)}}(r, q)$, então $\bar{g}^{(0)}(T, T)(r)=-\frac{d_{\bar{g}^{(0)}(p, q)^{2}}}{16}$. Se rescalonarmos $T^{a}$ por um fator $R \in \mathbb{R}, \kappa_{ \pm}$é rescalonada pelo mesmo fator, por definição. Isto tem, por exemplo, a conseqüência de que, se tomarmos $p_{0}=(-1,0), q_{0}=(1,0)$ e se rescalonarmos $T^{a}$ associado 
ao diamante $\mathscr{D}_{R p_{0}, R q_{0}}$ por um fator $\frac{2}{R}$, de modo a manter $\bar{g}^{(0)}(T, T)(r)=\eta(T, T)(0)=$ -1 , segue que $\kappa_{ \pm} \stackrel{R \rightarrow+\infty}{\longrightarrow} 0$, de forma consistente com o fato de que $\frac{2}{R} T^{a} \stackrel{R \rightarrow+\infty}{\longrightarrow}\left(\partial_{0}\right)^{a}$ (fisicamente, a "temperatura de UNRUH" associada às translações temporais no espaçotempo de Minkowski é zero).

Podemos entender a Proposição 1.8 como uma formulação da segunda lei da dinâmica de buracos negros para cunhas AAdS, pois o "encolhimento" das cunhas AAdS lá expresso provoca uma diminuição da área das seções transversais dos horizontes passados e futuros, tão maior quanto mais fundo penetramos no interior, ou seja, no sentido contrário ao da evolução temporal (note que, ao contrário do Teorema de Área de HAWKing, não podemos expressar este resultado diretamente em termos do espaçotempo físico $(\mathscr{M}, g)$, pois as seções transversais de horizontes de cunhas AAdS são não-compactas e destarte têm sempre área infinita). O caso em que não há aumento de entropia, que caracteriza processos reversíveis, fica:

TEOrEma 2.7 (CARACTERIZAÇÃo DE PROCESSOS REVERSíveis EM CUNHAS AAdS) Seja $(\mathscr{M}, g)$ um espaço-tempo AAdS assintoticamente simples satisfazendo as hipóteses da Proposição 1.8, e $(p, \bar{q}) \in \mathscr{D}(\mathscr{I}), r \in \mathscr{D}_{p, \bar{q}}$. Suponha que, para $r$ suficientemente próximo de $q$, temos que $\mathscr{W}_{r, \bar{q}}^{\prime} \cap \mathscr{W}_{q, \bar{r}}^{\prime}=\varnothing$. Então $\partial_{+} \mathscr{W}_{s, \bar{q}}$ é uma hipersuperfície tipo luz $\mathscr{C}^{\infty}$ e totalmente geodésica em relação a g para todo $s \in \mathscr{I}$ tal que $(s, \bar{q}) \in \mathscr{D}(\mathscr{I})$. Em particular, $\mathscr{W}_{s, \bar{q}}^{\prime} \cap \mathscr{W}_{q, \bar{s}}^{\prime}=\varnothing$ para todo $s \in \mathscr{I}$ tal que $(s, \bar{q}) \in \mathscr{D}(\mathscr{I})$.

Prova. $\quad$ Segue imediatamente da Proposição 1.8 e da Observação 1.7.

Note que, em virtude do Teorema 2.6, não faz sentido falar de uma "Terceira Lei" para a dinâmica de cunhas AAdS e diamantes. 


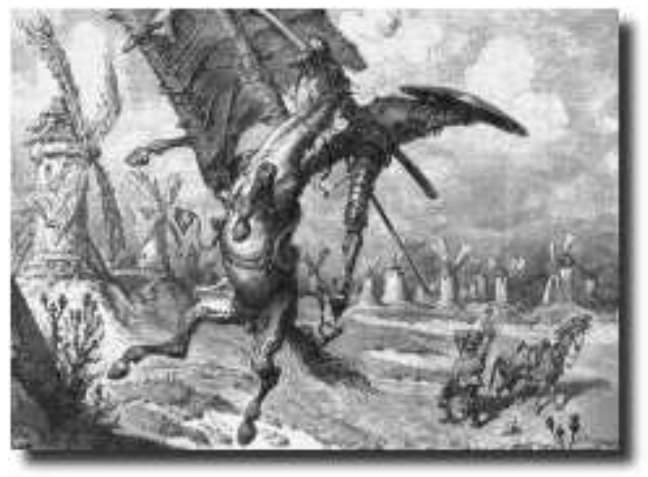

- A lo que yo imagino - dijo don Quijote -, no hay historia humana en el mundo que no tenga sus altibajos,

especialmente las que tratan de caballerías, las cuales nunca pueden estar llenas de prósperos sucesos. ${ }^{\mathrm{xi}}$

Miguel de Cervantes SaAvedra El ingenioso hidalgo don Quijote de la Mancha, Segundo Libro, Cap. III

\section{Avatares holográficos DA FísicA QUÂNTICA LOCAL}





\title{
CAPÍTUlO 3
}

\section{Teorias Quânticas Localmente Covariantes}

\author{
(HAMLET.) - O God! I could be bounded in \\ a nutshell, and count myself a king of infinite \\ space, were it not that I have bad dreams. ${ }^{\mathrm{xi}}$
}

William ShaKespeare Hamlet, Prince of Denmark ${ }^{x i i i}$, Ato II, Cena II

Havendo-nos convencido da necessidade de repensar a implementação de isometrias assintóticas do espaço-tempo já no contexto da gravitação clássica, introduziremos agora um formalismo adequado para a discussão desta questão no nível de teorias quânticas locais. Tal formalismo foi proposto recentemente por BRUNETTI, FredENHAGEN e VERCH [BFV03], e pode-se baseá-lo na observação, relacionada à ressalva no início do Capítulo 2 (ver página 32), de que a especificação de procedimentos físicos e seu ordenamento relativo são inerentemente locais - estes são insensíveis à estrutura do Universo como um todo, onde a região de localização destes procedimentos está mergulhada. Isso, como veremos, generaliza as noções de isotonia e covariância dos procedimentos físicos de uma só tacada. Podemos elevar esta observação à categoria de princípio - digamos, da covariância local.

Por outro lado, somos imediatamente forçados, à luz destas considerações, a considerar todas as regiões de todos os espaços-tempos possiveis em pé de igualdade. Mais precisamente, devemos especificar os procedimentos físicos possíveis de uma teoria para cada uma dessas regiões, de maneira coerente. Matematicamente, devemos especificar um funtor da categoria de regiões "admissíveis" de espaços-tempos na categoria de procedimentos físicos possíveis (orientamos o leitor não familiarizado com as noções de categoria e funtor a consultar o Apêndice C, página 149). É agora nosso objetivo precisar a estrutura que acabamos de esboçar, e investigar algumas de suas propriedades. 


\subsection{Definições básicas}

Primeiro, consideraremos uma teoria quântica localmente covariante sobre espaçostempos globalmente hiperbólicos, pois tal classe é "dinamicamente fechada" com respeito a equações de movimento que conduzem a uma propagação causal dos dados de CAUCHY. Neste caso, as relações de comutação causal entre observáveis podem ser unicamente determinadas (as modificações necessárias desse conceito em casos não globalmente hiperbólicos serão indicadas no Capítulo 4, página 69).

DeFiniçÃo 3.1 Sejam as categorias

$$
\mathscr{G} l h_{d}=\left\{\begin{aligned}
\text { objetos: } & \text { ObjG } l_{h_{d}}=\text { espaços-tempos }(\mathscr{M}, g) \text { d-dimensionais } \\
& \text { e globalmente hiperbólicos; } \\
\text { morfismos: }: & \text { Homglh } h_{d}\left((\mathscr{M}, g),\left(\mathscr{M}^{\prime}, g^{\prime}\right)\right)=\left\{\psi:(\mathscr{M}, g) \rightarrow\left(\mathscr{M}^{\prime}, g^{\prime}\right)\right. \\
& \text { mergulhos isométricos com imagem aberta e causalmente } \\
& \text { convexa }\} ;
\end{aligned}\right.
$$

$e$

$$
\mathscr{A} l g=\left\{\begin{aligned}
\text { objetos: } & \text { Obj } \mathscr{A} l g=C^{*} \text {-álgebras unitais } \mathfrak{A} ; \\
\text { morfismos: } & \operatorname{Hom}_{\mathscr{A} l g}\left(\mathfrak{A}, \mathfrak{A}^{\prime}\right)=\left\{\alpha: \mathfrak{A} \rightarrow \mathfrak{A}^{\prime}{ }^{*} \text {-monomorfismos unitais }\right\} .
\end{aligned}\right.
$$

Uma teoria quântica localmente covariante é um funtor covariante $\mathfrak{A}$ de $\mathscr{G} l h_{d}$ em $\mathscr{A} l g$.

Dizemos que $\mathfrak{A}:$

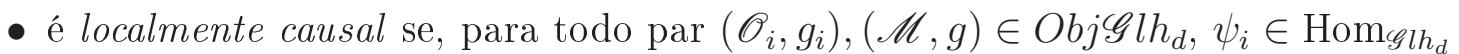
$\left(\left(\mathscr{O}_{i}, g_{i}\right),(\mathscr{M}, g)\right), i=1,2$, tal que $\psi_{1}\left(\mathscr{O}_{1}\right)$ e $\psi_{2}\left(\mathscr{O}_{2}\right)$ são causalmente disjuntos em $(\mathscr{M}, g)$, então

$$
\left[\left(\mathfrak{A} \psi_{1}\right)\left(\mathfrak{A}\left(\mathscr{O}_{1}, g_{1}\right)\right),\left(\mathfrak{A} \psi_{2}\right)\left(\mathfrak{A}\left(\mathscr{O}_{2}, g_{2}\right)\right)\right]=0
$$

em $\mathfrak{A}(\mathscr{M}, g)$.

- é primitivamente causal se, dados $(\mathscr{O}, g),(\mathscr{M}, g) \in \operatorname{Obj}^{C} l_{h} h_{d}, \psi \in \operatorname{Hom}_{\mathscr{G} l h_{d}}((\mathscr{O}$, $g),(\mathscr{M}, g))$, tais que $\psi(\mathscr{O})$ contém uma superfície de CAUCHY para $(\mathscr{M}, g)$, então $(\mathfrak{A} \psi)(\mathfrak{A}(\mathscr{O}, g))=\mathfrak{A}(\mathscr{M}, g)$, i.e., $\mathfrak{A} \psi$ é um $C^{*}$-isomorfismo.

- é aditivo se, dados $\left(\mathscr{O}_{i}, g_{i}\right) \in \operatorname{Obj} \mathscr{G}_{h} h_{d}, \psi_{i} \in \operatorname{Hom}_{\mathscr{g} l h_{d}}\left(\left(\mathscr{O}_{i}, g_{i}\right),(\mathscr{M}, g)\right), i \in I$, tais que a coleção $\left\{\psi_{i}\left(\mathscr{O}_{i}\right)\right\}$ é um recobrimento aberto de $(\mathscr{M}, g)$, então

$$
\overline{\bigvee_{i \in I}\left(\mathfrak{A} \psi_{i}\right)\left(\mathfrak{A}\left(\mathscr{O}_{i}, g_{i}\right)\right)}=\mathfrak{A}(\mathscr{M}, g)
$$

, onde $\vee_{i \in I}$ denota a *álgebra gerada pelas *álgebras indexadas por $i \in I$ e o fecho acima é na $\mathrm{C}^{*}$-norma de $\mathfrak{A}(\mathscr{M}, g)$. 
$\operatorname{Dados}\left(\mathscr{M}_{i}, g_{i}\right) \in \operatorname{Obj} \mathscr{G} l h, i=1,2$, tais que $\mathscr{M}_{1} \subset \mathscr{M}_{2}$ e $g_{1}=g_{2} \uparrow \mathscr{M}_{1}$, denotamos, para conveniência futura, por $i_{\mathscr{M}_{1}, \mathscr{M}_{2}}$ a inclusão natural de $\mathscr{M}_{1}$ em $\mathscr{M}_{2}$.

O leitor atento perceberá que poderíamos generalizar a Definição 3.1 de modo a incorporar qualquer configuração de campos externos clássicos a valores em fibrados sobre os objetos de $\mathscr{G} l h$, e não apenas a métrica. Um caso particular seriam os parâmetros dimensionais (massas, constantes de acoplamento, etc.) da teoria quântica de campos em questão. Essa possibilidade ficará mais evidente na Seção 3.4 (página 64, onde definimos campos quânticos localmente covariantes.

\subsection{Relação com os axiomas usuais}

Mostraremos agora como recuperar o esquema axiomático de HAAG e KASTLER [HK64, Haa96] para teorias de observáveis locais num espaço-tempo fixo a partir de uma teoria quântica localmente covariante $\mathfrak{A}$.

Considere um espaço-tempo fixo $(\mathscr{M}, g) \in O b j \mathscr{G} l h_{d}$, com grupo de isometrias (possivelmente trivial) $G(\mathscr{M}, g)$. Seja

$$
\mathscr{K}(\mathscr{M}, g) \doteq\{\mathscr{O} \subset \mathscr{M} \text { aberto e causalmente convexo }\} .
$$

Note que, pela definição (3.1), $\left(\mathscr{O}, g \uparrow_{\mathscr{O}}\right)$ é automaticamente globalmente hiperbólico para todo $\mathscr{O} \in \mathscr{K}(\mathscr{M}, g)$, em virtude da hiperbolicidade global de $(\mathscr{M}, g)$. Definindo

$$
\mathfrak{A}_{\mathscr{M}}(\mathscr{O}) \doteq \mathfrak{A} i_{\mathscr{O}, \mathscr{M}}\left(\mathfrak{A}\left(\mathscr{O}, g\left\lceil_{\mathscr{O}}\right)\right)\right.
$$

segue que

1. A correspondência $\mathscr{K}(\mathscr{M}, g) \ni \mathscr{O} \mapsto \mathfrak{A}_{\mathscr{M}}(\mathscr{O})$ é isótona, i.e., dados $\mathscr{O}_{1}, \mathscr{O}_{2} \in$ $\mathscr{K}(\mathscr{M}, g)$ tais que $\mathscr{O}_{1} \subset \mathscr{O}_{2}$, temos que $\mathfrak{A}_{\mathscr{M}}\left(\mathscr{O}_{1}\right) \subset \mathfrak{A}_{\mathscr{M}}\left(\mathscr{O}_{2}\right)$.

Para mostrar isto, note que

$$
\begin{aligned}
\mathfrak{A} i_{\mathscr{O}_{1}, \mathscr{O}_{2}}\left(\mathfrak { A } ( \mathscr { O } _ { 1 } , g \lceil _ { \mathscr { O } _ { 1 } } ) ) \subset \mathfrak { A } \left(\mathscr{O}_{2}, g\left\lceil\mathscr{O}_{2}\right)\right.\right. & \Rightarrow \\
\Rightarrow \mathfrak{A} i_{\mathscr{O}_{2}, \mathscr{M}}\left(\mathfrak{A} i_{\mathscr{O}_{1}, \mathscr{O}_{2}}\left(\mathfrak{A}\left(\mathscr{O}_{1}, g\left\lceil_{\mathscr{O}_{1}}\right)\right)\right)\right. & =\mathfrak{A} i_{\mathscr{O}_{1}, \mathscr{M}}\left(\mathfrak{A}\left(\mathscr{O}_{1}, g\left\lceil_{\mathscr{O}_{1}}\right)\right) \subset\right. \\
& \subset \mathfrak{A} i_{\mathscr{O}_{2}, \mathscr{M}}\left(\mathfrak{A}\left(\mathscr{O}_{2}, g\left\lceil_{\mathscr{O}_{2}}\right)\right),\right.
\end{aligned}
$$

onde a igualdade segue da covariância de $\mathfrak{A}$.

Em outras palavras, $\mathscr{K}(\mathscr{M}, g) \ni \mathscr{O} \mapsto \mathfrak{A}_{\mathscr{M}}(\mathscr{O})$ é um pré-cofeixe de $\mathrm{C}^{*}$-álgebras sobre os abertos causalmente convexos de $\mathscr{M}$, ordenados por inclusão (ver página 152 no Apêndice $\mathrm{C}$ para a definição categórica do conceito). Se $\mathscr{K}(\mathscr{M}, g)$ é direcionado (i.e., dados $\mathscr{O}_{1}, \mathscr{O}_{2} \in \mathscr{K}(\mathscr{M}, g)$, existe $\mathscr{O}_{3} \in \mathscr{K}(\mathscr{M}, g)$ tal que $\left.\mathscr{O}_{1}, \mathscr{O}_{2} \subset \mathscr{O}_{3}\right)$, então $\mathscr{K}(\mathscr{M}, g) \ni \mathscr{O} \mapsto \mathfrak{A}_{\mathscr{M}}(\mathscr{O})$ é uma rede. 
2. Existe uma representação de $G(\mathscr{M}, g) \ni u$ por ${ }^{*}$-automorfismos $\alpha_{u} \doteq \mathfrak{A} u$ de $\mathfrak{A}(\mathscr{M}, g)$ coerente com a estrutura de pré-cofeixe (rede) acima, isto é, $\alpha_{u_{1}} \circ \alpha_{u_{2}}=$ $\alpha_{u_{1} u_{2}}$ е $\alpha_{u}\left(\mathfrak{A}_{\mathscr{M}}(\mathscr{O})\right)=\mathfrak{A}_{\mathscr{M}}(u(\mathscr{O})), \forall u, u_{1}, u_{2} \in G(\mathscr{M}, g), \mathscr{O} \in \mathscr{K}(\mathscr{M}, g)$.

A primeira propriedade segue imediatamente da covariância de $\mathfrak{A}$. Para mostrar a segunda propriedade, notar que $u \uparrow_{\mathscr{O}}$ é um mergulho isométrico de $\left(\mathscr{O}, g \uparrow_{\mathscr{O}}\right) \mathrm{em}$ $\left(u(\mathscr{O}), g \uparrow_{u(\mathscr{O})}\right)$, e que $i_{\mathscr{O}, \mathscr{M}}=i_{u(\mathscr{O}), \mathscr{M}} \circ u$, para todo $u \in G(\mathscr{M}, g), \mathscr{O} \in \mathscr{K}(\mathscr{M}, g)$. O resultado segue então, uma vez mais, da covariância de $\mathfrak{A}$, como no item 1.

3. Se $\mathfrak{A}$ é localmente causal, segue que o pré-cofeixe (rede) $\mathscr{O} \mapsto \mathfrak{A}_{\mathscr{M}}(\mathscr{O})$ é localmente causal no sentido usual de HAAG e KAStler, i.e., dados $\mathscr{O}_{1}, \mathscr{O}_{2} \in \mathscr{K}(\mathscr{M}, g)$ tais que $\mathscr{O}_{1} \perp \mathscr{M} \mathscr{O}_{2}$, então $\left[\mathfrak{A}_{\mathscr{M}}\left(\mathscr{O}_{1}\right), \mathfrak{A}_{\mathscr{M}}\left(\mathscr{O}_{2}\right)\right]=\{0\}$ (segue imediatamente da Definição 3.1).

4. Analogamente ao item 3 , se $\mathfrak{A}$ for primitivamente causal (resp. aditivo), o précofeixe (rede) $\mathscr{O} \mapsto \mathfrak{A}_{\mathscr{M}}(\mathscr{O})$ é primitivamente causal (resp. aditivo) no sentido usual de HAAG e KASTLER.

Definição 3.2 Sejam $\mathfrak{A}$ uma teoria quântica localmente covariante, e $(\mathscr{M}, g) \in$ ObjGlh. O pré-cofeixe $\mathscr{K}(\mathscr{M}, g) \ni \mathscr{O} \mapsto \mathfrak{A}_{\mathscr{M}}(\mathscr{O})$ (rede, se $\mathscr{K}(\mathscr{M}, g)$ for direcionado) é dito ser uma realização de $\mathfrak{A}$ em $(\mathscr{M}, g)$.

Há um detalhe da realização de $\mathfrak{A}$ em $(\mathscr{M}, g)$ que requer certo cuidado em relação ao conceito de pré-cofeixe (rede) de observáveis locais de HAAG-KASTLER. Ele concerne a definição de álgebra quase-local: esta última é dada pelo limite indutivo (ver Apêndice C, fórmula C.1, página 154 para a definição categórica do conceito)

$$
\mathfrak{A}_{\mathscr{M}} \doteq \overline{\bigcup_{\mathscr{O} \in \mathscr{K}(\mathscr{M}, g)} \mathfrak{A}(\mathscr{O})^{\|\cdot\| \cdot \|}}
$$

onde $\|$.$\| é a norma de \mathfrak{A}(\mathscr{M}, g)$. Embora claramente $\mathfrak{A}_{\mathscr{M}} \subset \mathfrak{A}(\mathscr{M}, g)$, não é necessariamente verdade que $\mathfrak{A}_{\mathscr{M}}=\mathfrak{A}(\mathscr{M}, g)$ ! Não obstante, se ignorarmos $\mathfrak{A}(\mathscr{M}, g)$ e entendermos $\mathfrak{A}(\mathscr{O})$ como $C^{*}$-subálgebras locais de $\mathfrak{A}_{\mathscr{M}}$, então vemos que a realização de $\mathfrak{A}$ em $(\mathscr{M}, g)$ de fato define um pré-cofeixe de HAAG-KASTLER. Uma generalização do conceito de álgebra quase-local que permite definir teorias quânticas localmente covariantes em espaços-tempos não globalmente hiperbólicos e incorporar a especificação de condições de contorno será apresentada no Capítulo 4, página 69.

Vamos agora investigar algumas propriedades dinâmicas de $\mathfrak{A}$ primitivamente causal. Considere uma folheação de $(\mathscr{M}, g)$ por superfícies de CAUCHY $\mathbb{R} \ni t \mapsto \Sigma_{t}=$ $\tau^{-1}(t)$, dada pela função tempo global $\tau$ - mais precisamente, tal folheação é dada pelo difeomorfismo $F^{\tau}: \mathbb{R} \times \Sigma \ni(t, x) \mapsto F^{\tau}(t, x) \in \Sigma_{t} \subset \mathscr{M}$, onde convencionamos $\Sigma \doteq \Sigma_{0}$ (i.e., $F^{\tau}(t, \Sigma)=\Sigma_{t}$ e $F^{\tau}(\mathbb{R}, x)$ é a órbita de $x \in \Sigma_{0}$ induzida pela folheação). Atribua 
a cada $\Sigma_{t}$ uma vizinhança aberta e causalmente convexa $\mathscr{N}_{t} \supset \Sigma_{t}{ }^{1}$ e defina a família biparamétrica de ${ }^{*}$-isomorfismos [BF07, Ver96]

$$
\mathbb{R}^{2} \ni\left(t_{1}, t_{2}\right) \mapsto \alpha_{t_{1}, t_{2}}^{\tau} \doteq\left(\mathfrak{A} i_{\mathscr{N}_{t_{2}}, \mathscr{M}}\right)^{-1} \circ \mathfrak{A} i_{\mathscr{N}_{t_{1}}, \mathscr{M}}: \mathfrak{A}\left(\mathscr{N}_{t_{1}}, g \uparrow_{\mathscr{N}_{t_{1}}}\right) \rightarrow \mathfrak{A}\left(\mathscr{N}_{t_{2}}, g \uparrow_{\mathscr{N}_{t_{2}}}\right),
$$

denominada propagador de $\mathfrak{A}$ em $(\mathscr{M}, g)$ associado a $\tau$. O propagador $\alpha_{., \text {, }}^{\tau}$ satisfaz

$$
\alpha_{t_{2}, t_{3}}^{\tau} \circ \alpha_{t_{1}, t_{2}}^{\tau}=\alpha_{t_{1}, t_{3}}^{\tau}, \alpha_{t, t}^{\tau}=\operatorname{id}_{\mathfrak{A}(\mathscr{M}, g)}, \forall t_{1}, t_{2}, t_{3}, t \in \mathbb{R}
$$

i.e., $\alpha_{.,}^{\tau}$ implementa o grupóide de pares $\mathscr{P} \mathbb{R}$ associado a $\mathbb{R} .^{2}$ É importante notar que, embora o propagador preserve, por definição, a localização dos procedimentos físicos ao longo do tempo (i.e., das órbitas da folheação), ele não implementa de maneira geométrica o fluxo de difeomorfismos $F(.+t,.) \circ F^{-1}, t \in \mathbb{R}$ : considere, por exemplo, um aberto $\mathscr{S}$ de $\Sigma$, e defina $\mathscr{S}_{t} \doteq F^{\tau}(t, \mathscr{S})$. É falso que $\alpha_{t_{1}, t_{2}}^{\tau}\left(\mathfrak{A}_{\mathscr{N}_{1}}\left(D\left(\mathscr{S}_{t_{1}}\right) \cap \mathscr{N}_{t_{1}}, g \uparrow\right.\right.$ $\left.\left.D\left(\mathscr{S}_{t_{1}}\right) \cap \mathscr{N}_{t_{1}}\right)\right)=\mathfrak{A}_{\mathscr{N}_{t_{2}}}\left(D\left(\mathscr{S}_{t_{2}}\right) \cap \mathscr{N}_{t_{2}}, g\left\lceil D\left(\mathscr{S}_{t_{2}}\right) \cap \mathscr{N}_{t_{2}}\right)(D(\right.$.$) denota o desenvolvimento de$ CAUCHY; note que a intersecção de dois abertos causalmente convexos é causalmente convexa), indicando que $\alpha_{.,}^{\tau}$ é, de fato, um objeto dinâmico e não cinemático.

Ressaltamos também que o tamanho de $\mathscr{N}_{t}$ é essencialmente irrelevante. De fato, podemos tomar o conjunto $\mathfrak{N}_{t}$ de todas as vizinhanças abertas e causalmente convexas de $\Sigma_{t}$ e definir os limites projetivos (ver Apêndice C, fórmula C.1, página 154 para a definição categórica do conceito)

$$
\begin{aligned}
\mathfrak{A}_{\Sigma_{t}} & \doteq \bigcup_{\mathscr{N}_{t} \in \mathfrak{N}_{t}} \mathfrak{A}\left(\mathscr{N}_{t}, g \uparrow_{\mathscr{N}_{t}}\right) / \sim \\
\mathfrak{A}_{\Sigma_{t}}\left(\mathscr{S}_{t}\right) & \doteq \bigcup_{\mathscr{N}_{t} \in \mathfrak{N}_{t}} \mathfrak{A}_{\mathscr{N}_{t}}\left(D\left(\mathscr{S}_{t}\right) \cap \mathscr{N}_{t},\left.g\right|_{D\left(\mathscr{S}_{t} \cap \mathscr{N}_{t}\right)}\right) / \sim^{\prime}, \mathscr{S} \subset \Sigma \text { aberto, }
\end{aligned}
$$

onde a relação de equivalência $\sim$ identifica os elementos das órbitas da família de *_ isomorfismos $\mathfrak{A} i_{\mathscr{N}_{t}^{1}, N_{t}^{2}}$ e a relação de equivalência $\sim^{\prime}$ identifica os elementos das órbitas da família de ${ }^{*}$-isomorfismos $\mathfrak{A} i_{D\left(\mathscr{S}_{t}\right) \cap \mathscr{N}_{t}^{1}, D\left(\mathscr{S}_{t}\right) \cap \mathscr{N}_{t}^{2}}, \mathscr{N}_{t}^{i} \in \mathfrak{N}_{t}, i=1,2, \mathscr{N}_{t}^{1} \subset \mathscr{N}_{t}^{2}$. Esse procedimento define a álgebra quase-local e as subálgebras locais de germes de

\footnotetext{
${ }^{1}$ Note que não definimos primeiro $\mathscr{N}_{t_{0}}$ para um $t_{0}$ fixo para depois definir $\mathscr{N}_{t}$ como a imagem de $\mathscr{N}_{t_{0}}$ pelo fluxo de $t_{0}$ a $t$ induzido pela folheação. Como esta família de difeomorfismos não necessariamente preserva a estrutura causal de $\mathscr{N}_{t_{0}}$ - para tal, é necessário e suficiente que os difeomorfismos sejam aqui transformações conformes [BEE96] -, a imagem de $\mathscr{N}_{t_{0}}$ pode não ser causalmente convexa. Ademais, é por vezes conveniente tratar duas superfícies de CAUCHY disjuntas sem que, necessariamente, tenhamos o fluxo de uma folheação conectando ambas; uma situação em que isto ocorre será ilustrada mais adiante.

${ }^{2}$ Dado um conjunto $S$, o grupóide de pares $\mathscr{P} S$ associado a $S$ é definido da seguinte forma (para a definição de grupóide, ver o Apêndice C, página 151): $\operatorname{Obj} \mathscr{P} S \doteq S, \operatorname{Arr} \mathscr{P} S=S^{2} \ni\left(s_{1}, s_{2}\right)$, onde $D\left(\left(s_{1}, s_{2}\right)\right)=s_{1}, C D\left(\left(s_{1}, s_{2}\right)\right)=s_{2}, \mathbb{1}_{s}=(s, s)$ e a lei de composição é dada por $\left(s_{2}, s_{3}\right)\left(s_{1}, s_{2}\right)=$ $\left(s_{1}, s_{3}\right)$.
} 
procedimentos físicos no instante $t$ em relação à folheação induzida por $\tau$. Denotamos também por $\alpha_{t_{1}, t_{2}}^{\tau}$ o propagador ligando $\mathfrak{A}_{\Sigma_{t_{1}}}$ a $\mathfrak{A}_{\Sigma_{t_{2}}}$ induzido por (3.6). Note que o limite projetivo não é a "restrição de $\mathfrak{A}(\mathscr{M}, g)$ a $\Sigma_{t}$ " (tal identificação só é possível para teorias quânticas de campo livres, para as quais a restrição a hipersuperfícies tipo espaço é uma operação matematicamente bem definida).

\subsection{Estados quânticos em espaços-tempos curvos}

Já a noção de estados requer mais cuidado neste formalismo, pois, ao contrário dos procedimentos físicos, os estados são inerentemente não-locais - tanto são sensíveis à geometria global do espaço-tempo como podem responder de maneira não-local a perturbações locais da métrica.

DEFIniÇÃo 3.3 Seja a categoria

$$
\mathscr{S} t s=\left\{\begin{aligned}
\text { objetos: } & \text { Obj } \mathscr{S} t s=\text { conjuntos } \mathfrak{S} \text { de estados sobre } C^{*} \text {-álgebras } \\
& \text { unitais; } \\
\text { morfismos: } & \text { Hom } \operatorname{Sots}_{t s}\left(\mathfrak{S}, \mathfrak{S}^{\prime}\right)=\left\{\sigma: \mathfrak{S} \rightarrow \mathfrak{S}^{\prime} \text { aplicações lineares positivas }\right\} .
\end{aligned}\right.
$$

Dada uma teoria quântica localmente covariante $\mathfrak{A}$, um espaço de estados (localmente contravariante) é um funtor contravariante $\mathfrak{S}$ de $\mathscr{G} l h_{d}$ em $\mathscr{S} t s$, tal que $\mathfrak{S} \psi=(\mathfrak{A} \psi)^{*}$ é o dual de $\mathfrak{A} \psi$ para todo $\psi \in \operatorname{Arr} \mathscr{G} l h$, i.e., dado $\omega \in \mathfrak{S}\left(\mathscr{M}^{\prime}, g^{\prime}\right)$ e $\psi \in \operatorname{Hom}_{\mathscr{G}}{ }_{\mathrm{l} h}\left((\mathscr{M}, g),\left(\mathscr{M}^{\prime}, g^{\prime}\right)\right)$, temos que $\mathfrak{S} \psi \in \operatorname{Hom}_{\mathscr{S t s}}\left(\mathfrak{S}\left(\mathscr{M}^{\prime}, g^{\prime}\right), \mathfrak{S}(\mathscr{M}, g)\right)$ é definido por $\mathfrak{S} \psi(\omega)=(\mathfrak{A} \psi)^{*}(\omega) \doteq$ $\omega \circ \mathfrak{A} \psi$.

\subsection{Campos quânticos localmente covariantes}

Nos desenvolvimentos apresentados até agora, a estrutura topológica de $\mathrm{C}^{*}$-álgebras atribuída aos objetos de $\mathscr{A} l g$ teve apenas um papel secundário (definição de álgebras quase-locais, etc.). De fato, poderíamos ter definido os objetos de $\mathscr{A} l g$ como *álgebras unitais, e atribuir uma estrutura topológica conforme a conveniência. Antes de prosseguir, definamos a categoria

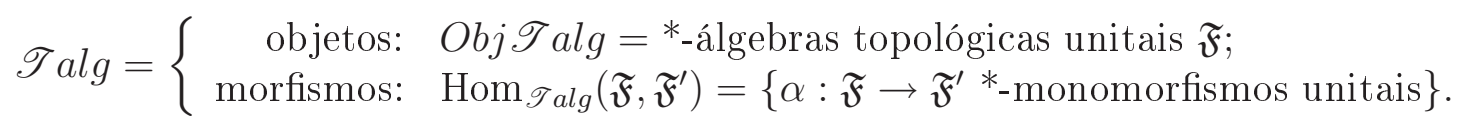

e o funtor de "pushforward" de funções teste $\mathfrak{D}: \mathscr{G} l h_{d} \rightarrow \mathscr{T} v s$ dado por

$$
\begin{aligned}
\mathfrak{D}(\mathscr{M}, g) & =\mathscr{C}_{c}^{\infty}(\mathscr{M}) \\
\mathfrak{D} \psi(f)(p) & =\psi_{*}(f)(p) \doteq\left\{\begin{array}{lr}
f(q) & (p=\psi(q)) \\
0 & \text { de outra forma }
\end{array}\right.
\end{aligned}
$$


onde $\mathscr{T}$ vs é a categoria de espaços vetoriais topológicos (ver o Apêndice C). Podemos também, eventualmente, entender $\mathfrak{D}$ como um funtor de $\mathscr{G} l h$ na categoria $\mathscr{T}$ op de espaços topológicos.

DEFINIÇÃO 3.4 Uma teoria quântica de campos localmente covariante é um funtor covariante $\mathfrak{F}: \mathscr{G} l h \rightarrow \mathscr{T}$ alg. Um campo quântico escalar localmente covariante é uma transformação natural $\Phi: \mathfrak{D} \rightarrow \mathfrak{F}$, onde $\mathfrak{D}$ e $\mathfrak{F}$ são aqui entendidos como funtores de $\mathscr{G}$ lh em $\mathscr{T}$ op. Dizemos que $\Phi$ é linear se $\Phi_{(\mathscr{M}, g)} \in \operatorname{Arr} \mathscr{T}$ vs para todo $(\mathscr{M}, g) \in$ ObjĞlh.

OBSERVAÇÃo 3.5 Note que a hipótese de $\Phi_{(\mathscr{M}, g)}$ ser linear no sentido da Definição 3.4 nada tem a ver com a natureza de sua auto-interação!

As noções de causalidade local, causalidade primitiva, aditividade, realização de $\mathfrak{F}$ em $(\mathscr{M}, g) \in O b j \mathscr{G} l h$ e de espaço de estados são obtidas naturalmente como extensões dos conceitos correspondentes para teorias quânticas localmente covariantes (a única modificação necessária é a substituição de "C*-álgebra" por "**álgebra" na fórmula (3.7) da Definição 3.3, página 64).

Um exemplo típico (ainda que aparentemente trivial) de teoria quântica de campos localmente covariante é a atribuição, a cada $(\mathscr{M}, g) \in O b j \mathscr{G} l h$, da álgebra de Borchers-Uhlmann $\mathscr{F}(\mathscr{M})$ (ver Apêndice B.3, Definição B.11, página 146) - é imediato ver que $\mathfrak{F}$ satisfaz a Definição 3.4. Esta teoria quântica de campos localmente covariante serve unicamente para definir a cinemática de um campo escalar hermitiano, e é denominada doravante funtor de Borchers-UHLMANn. A realização deste $\mathfrak{F}$ em $(\mathscr{M}, g) \in O b j \mathscr{G}$ lh possui como álgebra quase-local $\mathfrak{F}_{\mathscr{M}}=\mathfrak{F}(\mathscr{M})$ (basta aplicar partições de unidade), e exemplos possíveis de campos quânticos escalares localmente covariantes associados a $\mathfrak{F}$ são:

- $\Phi_{(\mathscr{M}, g)}=\operatorname{id}_{\mathscr{C}_{c}^{\infty}(\mathscr{M})}$, i.e, $\Phi$ identifica $f \in \mathscr{C}_{c}^{\infty}(\mathscr{M})$ com o elemento correspondente de $\mathfrak{F}(\mathscr{M})$;

- $\Phi_{(\mathscr{M}, g)}(f)=[f]$, onde $[f]$ é a classe de equivalência de $f$ pelo quociente de $\mathfrak{F}(\mathscr{M})$ por um *-ideal $\mathfrak{I}(\mathscr{M}, g)$. Para que $(\mathscr{M}, g) \mapsto \mathfrak{F}(\mathscr{M}) / \mathfrak{I}(\mathscr{M}, g)$ defina uma teoria quântica de campos localmente covariante, é necessário que a correspondência $(\mathscr{M}, g) \mapsto \mathfrak{I}(\mathscr{M}, g)$ defina um funtor covariante de $\mathscr{G} l h \mathrm{em} \mathscr{T}$ alg. Neste caso, a aplicação quociente define uma transformação natural, em virtude de sua propriedade universal [Jac85] (ver também o Apêndice C, página 146). A especificação de *-ideais como $\mathfrak{I}(\mathscr{M}, g)$ constituem uma maneira de impor uma dinâmica à álgebra de Borchers-Uhlmann. Tanto este exemplo como o anterior resultam em campos lineares. 
- Aqui elaboramos o exemplo anterior, baseando-nos nas considerações no final da Seção B.3. Considere um espaço de estados $\mathfrak{S}$, onde $\mathfrak{S}(\mathscr{M}, g)$ consiste num conjunto de hierarquias $\omega$ de distribuições de $k$ pontos $\omega_{k} \in \mathscr{D}^{\prime}\left(\mathscr{M}^{k}\right)$ e $\mathfrak{S} \psi$ consiste no "pullback" $\psi^{*}$ de distribuições por $\psi \in \operatorname{Arr} \mathscr{G} l h$, tal que

$$
\Im(\mathscr{M}, g) \subset \bigcap_{\omega \in \mathfrak{S}(\mathscr{M}, g)} \text { Ann } \omega .
$$

Neste caso, o campo quântico localmente covariante $\Phi$ do exemplo anterior induz naturalmente um conjunto de estados $\Phi_{(\mathscr{M}, g)}^{*} \mathfrak{S}(\mathscr{M}, g)$ em cada $\widetilde{\mathfrak{F}}(\mathscr{M}, g) \doteq$ $\mathfrak{F}(\mathscr{M}) / \mathfrak{I}(\mathscr{M}, g)$

$$
\left(\Phi_{(\mathscr{M}, g)}^{*} \omega_{k}\right)\left(\left[f_{1}\right], \ldots,\left[f_{k}\right]\right)=\omega_{k}\left(f_{1}, \ldots, f_{k}\right),
$$

de modo que $\Phi^{*} \mathfrak{S}($.$) é um espaço de estados localmente contravariante.$

A possibilidade de proceder como no formalismo de Wightman e definir campos quânticos a partir de estados por meio da representação WiGHTMAN-GNS pode, é claro, ser feita para a realização do funtor de BorChERS-Uhlmann em um $(\mathscr{M}, g) \in \mathscr{G} l h$ qualquer, mas deve ser considerada com cuidado no que concerne o Princípio de Covariância Local, uma vez que estados individuais não são localmente contravariantes. Podemos, todavia, considerar a seguinte situação - por sinal, já considerada por HAAG e KASTLER em [HK64], e que lhes serviu de motivação para a introdução da abordagem algébrica à TQC - à luz dos exemplos tratados acima.

Seja $\mathfrak{S}$ um espaço de estados associado a $\mathfrak{F}$, tal que

$$
\operatorname{Ann} \omega_{(\mathscr{M}, g)}=\operatorname{Ann} \omega_{(\mathscr{M}, g)}^{\prime} \doteq \mathfrak{I}(\mathscr{M}, g), \forall \omega_{(\mathscr{M}, g)}, \omega_{(\mathscr{M}, g)}^{\prime} \in \mathfrak{S}(\mathscr{M}, g),(\mathscr{M}, g) \in \text { ObjGlh }
$$

Empregando a contravariância de $\mathfrak{S}$, podemos mostrar que, neste caso, a correspondência $(\mathscr{M}, g) \mapsto \mathfrak{I}(\mathscr{M}, g)$ define uma teoria quântica de campos localmente covariante. Portanto, como vimos, $\Phi_{(\mathscr{M}, g)}(f)=[f]=f / \mathfrak{I}(\mathscr{M}, g)$ define então um campo escalar localmente covariante, realizando assim nosso desideratum de incorporar a informação dinâmica de uma teoria quântica de campos localmente covariante na especificação de um espaço de estados, mostrando ao mesmo tempo que o campo quântico obtido é independente da escolha de um representante do conjunto de estados, pelo Primeiro Teorema de *-Isomorfismo.

Do ponto de vista de uma teoria quântica localmente covariante $\mathfrak{A}$, (3.8) possui uma interpretação ainda mais profunda: as representações GNS de $\mathfrak{A}(\mathscr{M}, g)$ associadas a cada $\omega_{(\mathscr{M}, g)} \in \mathfrak{S}(\mathscr{M}, g)$ não apenas são quase-equivalentes entre si (i.e., os conjuntos de estados normais em $\pi_{\omega_{(\mathscr{M}, g)}}(\mathfrak{A}(\mathscr{M}, g))^{\prime \prime}$ e $\pi_{\omega_{(\mathscr{M}, g)}^{\prime}}(\mathfrak{A}(\mathscr{M}, g))^{\prime \prime}$ coincidem, ou, equivalentemente, essas duas álgebras de vON NeumanN são *-isomorfas - ver o Teorema 2.4.26 
de [BR87]), como também, por conseguinte, as representações GNS da álgebra quaselocal $\mathfrak{A}_{\mathscr{M}}$ da realização de $\mathfrak{A}$ em qualquer $(\mathscr{M}, g)$ são localmente quase-equivalentes entre si, ou seja, os conjuntos de estados normais em $\pi_{\omega_{(\mathscr{M}, g)}}(\mathfrak{A}(\mathscr{O}))^{\prime \prime}$ e $\pi_{\omega_{(\mathscr{M}, g)}^{\prime}}(\mathfrak{A}(\mathscr{O}))^{\prime \prime}$ coincidem. Assim, quase-equivalência local torna-se um requerimento altamente desejável para espaços de estados fisicamente relevantes e nos permite essencialmente passar de $C^{*}$-álgebras locais para álgebras de VON NEUMAnN locais da mesma maneira que no formalismo de HAAG-KASTLER. Uma discussão mais detalhada acerca do papel da quase-equivalência local no contexto do Princípio de Covariância Local pode ser encontrada em [BFV03]. 



\section{CAPÍtulo 4}

\section{Implementando o Princípio Holográfico em espaços-tempos AAdS}

In due modi si raggiunge Despina: per nave o per cammello. La città si presenta differente a chi viene da terra e a chi dal mare.

(...)

Ogni città riceve la sua forma dal deserto a cui si oppone; e cosi il cammelliere e il marinaio vedono Despina, città di confine tra due deserti. ${ }^{\text {xiv }}$

ItALO CALVino

"Le città e il desiderio $3 "$ " Le città invisibili $)^{\mathrm{xv}}$

\subsection{Covariância local com condições de contorno}

No Capítulo 3, construímos um arcabouço geral para a descrição de teorias quânticas localmente covariantes dentro de espaços-tempos globalmente hiperbólicos. Em tal classe, relações de comutação entre os procedimentos locais (dados pelos elementos das álgebras locais) podem ser estabelecidas sem ambigüidades pois espaços-tempos globalmente hiperbólicos constituem sistemas "fechados" com respeito a leis dinâmicas causais, i.e., que propagam efeitos físicos locais com velocidade inferior ou igual à da luz. Entretanto, sabemos que podemos sempre recobrir qualquer espaço-tempo com vizinhanças abertas globalmente hiperbólicas e causalmente convexas - no caso de espaços-tempos fortemente causais, tais vizinhanças constituem inclusive uma base para a topologia do espaço-tempo. E nem por isso devemos esperar que, nesses sistemas 
"abertos", possamos assumir causalidade local a priori.

No caso da realização de $\mathfrak{A}$ num espaço-tempo globalmente hiperbólico, a causalidade local é coerente com as inclusões das álgebras, pois mediante uma escolha adequada da família de vizinhanças globalmente hiperbólicas, sempre é possível encontrar, dadas duas tais vizinhanças causalmente disjuntas (sendo, eventualmente, uma delas suficientemente pequena), uma terceira que contenha ambas. ${ }^{1}$ É precisamente a violação desta propriedade que causa dificuldades no caso de espaços-tempos não globalmente hiperbólicos.

Para precisar melhor tais dificuldades na extensão do Princípio de Covariância Local a espaços-tempos tais como (A)AdS, exibiremos agora uma maneira mais geral de construir a realização de uma teoria quântica localmente covariante $\mathfrak{A}$, válida inclusive para espaços-tempos $(\mathscr{M}, g)$ não globalmente hiperbólicos. Esta maneira foi proposta por SOMMER [Som06] e torna mais transparentes a necessidade e o papel das condições de contorno na imposição de causalidade local e causalidade primitiva - para tornar mais claro o contexto desta última propriedade, restringiremo-nos a espaços-tempos estavelmente causais, pois estes, como visto no Apêndice A, podem ser folheados por hipersuperfícies "de tempo constante", para as quais tem significado formular um problema de valor inicial (misto).

Seja, então, um espaço-tempo estavelmente causal $(\mathscr{M}, g)$, e $\mathscr{K}(\mathscr{M}, g)$ o conjunto das regiões abertas globalmente hiperbólicas e causalmente convexas $\left(\mathscr{O}, g \int_{\mathscr{O}}\right), \mathscr{O} \subset$ $\mathscr{M}$ (eventualmente, omitiremos $g \Gamma_{\mathscr{O}}$ quando tal procedimento não causar confusão). Consideremos inicialmente, dada uma teoria quântica localmente covariante $\mathfrak{A}$,

$$
\mathfrak{A}_{\mathscr{M}}=\bigvee\left\{(A, \mathscr{O}): A \in \mathfrak{A}\left(\mathscr{O}, g \uparrow_{\mathscr{O}}\right), \mathscr{O} \in \mathscr{K}(\mathscr{M}, g)\right\}
$$

a *álgebra livre gerada pelos elementos indicados acima. Seja agora o *-ideal $\mathfrak{I}_{\mathscr{M}} \doteq$ $\mathfrak{A}_{\mathscr{M}} \mathfrak{E}_{\mathscr{M}} \mathfrak{A}_{\mathscr{M}}$, onde $\mathfrak{E}_{\mathscr{M}}$ é a ${ }^{*}$-álgebra gerada pelas relações

$$
\begin{aligned}
& (A, \mathscr{O})+(B, \mathscr{O})-(A+B, \mathscr{O}) \\
& (A, \mathscr{O})(B, \mathscr{O})-(A B, \mathscr{O}), \\
& \lambda(A, \mathscr{O})-(\lambda A, \mathscr{O}), \forall \lambda \in \mathbb{C} \mathrm{e} \\
& \left(A, \mathscr{O}_{1}\right)-\left(\mathfrak{A} i_{\mathscr{O}_{1}, \mathscr{O}_{2}}(A), \mathscr{O}_{2}\right), \forall \mathscr{O}, \mathscr{O}_{1}, \mathscr{O}_{2} \in \mathscr{K}(\mathscr{M}, g), \mathscr{O}_{1} \subset \mathscr{O}_{2} .
\end{aligned}
$$

Sabemos que cada elemento $(A, \mathscr{O})$ possui $\left(\mathrm{C}^{*}-\right)$ norma $\|(A, \mathscr{O})\|=\|A\|$, consistente com as relações (4.2) (lembrar que $\mathfrak{A} i_{\mathscr{O}_{1}, \mathscr{O}_{2}}$ é um ${ }^{*}$-monomorfismo para $\mathscr{O}_{1}, \mathscr{O}_{2} \in$

\footnotetext{
${ }^{1}$ Ver [GLRV01]. Esse fato é de suma importância na Teoria Algébrica de Setores de Superseleção.
} 
$\mathscr{K}(\mathscr{M}, g)$, e, portanto, $\left.\left\|\left(\mathfrak{A} i_{\mathscr{O}_{1}, \mathscr{O}_{2}}(A), \mathscr{O}_{2}\right)\right\|=\left\|\left(A, \mathscr{O}_{1}\right)\right\|=\|A\| !\right)$. Empregando agora a construção universal de BLACKADAR [Bla85, Bla06], ${ }^{2}$ aos geradores em (4.1) e às relações (4.2), definimos a coleção $\Pi\left(\mathfrak{A}_{\mathscr{M}}, \mathfrak{I}_{\mathscr{M}}\right)$ das ${ }^{*}$-representações admissiveis do par $\left(\mathfrak{A}_{\mathscr{M}}, \mathfrak{I}_{\mathscr{M}}\right)$, consistindo das ${ }^{*}$-representações $\pi$ de $\mathfrak{A}_{\mathscr{M}}$ tais que $\pi\left(\mathfrak{I}_{\mathscr{M}}\right)=\{0\}$. Segue então que a $\mathrm{C}^{*}$-seminorma universal $\|A\|_{u}=\sup _{\pi \in \Pi\left(\mathfrak{A}_{\mathscr{M}}, \mathfrak{I}_{\mathscr{M}}\right)}\|\pi(A)\|$ é finita para todo $A$. Denotando o ${ }^{*}$-ideal $\overline{\mathfrak{I}}_{\mathscr{M}} \doteq\left\{A:\|A\|_{u}=0\right\}$, obtemos então a $\mathrm{C}^{*}$-álgebra quase-local $\mathfrak{A}(\mathscr{M}, g)$ como a $\mathrm{C}^{*}$-álgebra universal dada pelos geradores $\mathfrak{A}_{\mathscr{M}}$ e relações $\mathfrak{I}_{\mathscr{M}}:^{3}$

$$
\mathfrak{A}(\mathscr{M}, g) \doteq \overline{\left(\mathfrak{A}_{\mathscr{M}} / \overline{\mathfrak{I}}_{\mathscr{M}}\right)^{\|\cdot\|_{u}}}
$$

Vamos ver agora como ficam os ${ }^{*}$-morfismos induzidos por mergulhos isométricos $\psi$ : $\left(\mathscr{M}_{1}, g_{1}\right) \rightarrow\left(\mathscr{M}_{2}, g_{2}\right)$ com imagem causalmente convexa. Obviamente, $\psi\left(\mathscr{K}\left(\mathscr{M}_{1}, g_{1}\right)\right) \subset$ $\mathscr{K}\left(\mathscr{M}_{2}, g_{2}\right)$, e $\psi \uparrow_{\mathscr{O}}, \mathscr{O} \in \mathscr{K}\left(\mathscr{M}_{1}, g_{1}\right)$, define um mergulho isométrico de $\mathscr{O}$ em $\psi(\mathscr{O})$, definindo naturalmente um ${ }^{*}$-monomorfismo de $\mathfrak{A}_{\mathscr{M}_{1}}$ em $\mathfrak{A}_{\mathscr{M}_{2}}$. Definamos o seguinte *-morfismo:

$$
\begin{aligned}
\mathfrak{A} \psi: \mathfrak{A}\left(\mathscr{M}_{1}, g_{1}\right) & \rightarrow \mathfrak{A}\left(\mathscr{M}_{2}, g_{2}\right) \\
A+\overline{\mathfrak{I}}_{\mathscr{M}_{1}} & \mapsto \mathfrak{A} \psi(A)+\overline{\mathfrak{I}}_{\mathscr{M}_{2}} .
\end{aligned}
$$

Note que, em razão das considerações do parágrafo anterior, temos que, se $[A]_{\mathscr{M}_{i}}$ é a classe de equivalência de $A \in \mathfrak{A}_{\mathscr{M}_{i}}$ pelo quociente por $\overline{\mathfrak{I}}_{\mathscr{M}_{i}}$ (i.e., $[A]_{\mathscr{M}_{i}}=[A+J]_{\mathscr{M}_{i}}$ se $\left.J \in \overline{\mathfrak{I}}_{\mathscr{M}_{i}}\right), i=1,2$, segue que $\mathfrak{A} \psi\left([A+J]_{\mathscr{M}_{1}}\right)=\left[\mathfrak{A} \psi \Gamma_{\mathscr{O}}(A)\right]_{\mathscr{M}_{2}}$, para todo $A \in \mathfrak{A}\left(\mathscr{O}, g\left\lceil_{\mathscr{O}}\right)\right.$, mostrando que a definição (4.4) não é vazia e define de fato um *-morfismo não-trivial. $\mathfrak{A} \psi$ é unital, mas não é necessariamente injetivo! Assim, temos que enfraquecer as propriedades dos morfismos da categoria de $(\mathrm{C})^{*}$-álgebras para que $\mathfrak{A}$ defina um funtor covariante da (super)categoria (plena) dos espaços-tempos estavelmente causais nesta última - a covariância de $\mathfrak{A}$ é garantida pelo Primeiro Teorema de *-Isomorfismo (Teorema B.2, página 125). Exibiremos agora uma situação que ilustra com perfeição como esse fato complica a imposição da causalidade local.

Considere $\mathscr{O}_{1}, \mathscr{O}_{2} \in \mathscr{K}\left(\mathscr{M}_{1}, g_{1}\right)$ causalmente disjuntos, tais que não existe $\mathscr{O}_{3} \in$ $\mathscr{K}\left(\mathscr{M}_{1}, g_{1}\right)$ tal que $\mathscr{O}_{1}, \mathscr{O}_{2} \subset \mathscr{O}_{3}$, mas existe $\mathscr{O}_{4} \in \mathscr{K}\left(\mathscr{M}_{2}, g_{2}\right)$ tal que $\psi\left(\mathscr{O}_{1}\right), \psi\left(\mathscr{O}_{2}\right) \subset$ $\mathscr{O}_{4}$. Seja $\mathfrak{A}$ localmente causal (i.e., com respeito a regiões globalmente hiperbólicas). Então, temos que

$$
\left[\left(\mathfrak{A}\left(i_{\psi\left(\mathscr{O}_{1}\right), \mathscr{O}_{4}} \circ \psi\right)(A), \mathscr{O}_{4}\right),\left(\mathfrak{A}\left(i_{\psi\left(\mathscr{O}_{2}\right), \mathscr{O}_{4}} \circ \psi\right)(B), \mathscr{O}_{4}\right)\right]=0
$$

\footnotetext{
${ }^{2}$ Agradeço ao Prof. Severino Toscano do Rêgo Melo por trazer a referência [Bla85] à minha atenção.

${ }^{3}$ Note que não tomamos o quociente pelo *-ideal $\mathfrak{I}_{\mathscr{M}}$. Caso houvesse uma maneira de garantir a existência de uma *-representação admissivel $\pi$ tal que $\operatorname{Ker} \pi=\mathfrak{I}_{\mathscr{M}}$, poderíamos equivalentemente tomar tal quociente. Todavia, isso em geral é impossível, pois $\mathfrak{I} \mathscr{M}$ não é necessariamente fechado na $\mathrm{C}^{*}$-norma definida nos elementos de $\mathfrak{A}_{\mathscr{M}}$.
} 
Entretanto, nada sob nossas hipóteses impede que

$$
\left[\left(A, \mathscr{O}_{1}\right),\left(B, \mathscr{O}_{2}\right)\right]=C \neq 0
$$

a menos que $A=0$ ou $B=0$. Assim, para $A, B \neq 0$, segue que $C \notin \mathfrak{I}_{\mathscr{M}_{1}} \mathrm{e}$ $[C]_{\mathscr{M}_{1}} \in \operatorname{Ker} \mathfrak{A} \psi$, potencialmente espoliando a injetividade de $\mathfrak{A} \psi$ (pode ocorrer, contudo, que $C \in \overline{\mathfrak{I}}_{\mathscr{M}_{1}}$, mas não há como garantir isso a priori). Não obstante, os *morfismos $\mathfrak{A}\left(\psi \circ i_{\mathscr{O}, \mathscr{M}_{1}}\right)$ são sempre injetivos, para todo $\mathscr{O} \in \mathscr{K}\left(\mathscr{M}_{1}, g_{1}\right)$.

A construção acima permite escrever a seguinte

DEFIniÇÃo 4.1 Sejam as categorias

$$
\mathscr{S} t c_{d}=\left\{\begin{aligned}
\text { objetos: } & \text { ObjĞl } h_{d}=\text { espaços-tempos }(\mathscr{M}, g) \text { d-dimensionais } \\
& \text { e estavelmente causais; } \\
\text { morfismos: }: & \text { Hom } \text { stc }_{d}\left((\mathscr{M}, g),\left(\mathscr{M}^{\prime}, g^{\prime}\right)\right)=\left\{\psi:(\mathscr{M}, g) \rightarrow\left(\mathscr{M}^{\prime}, g^{\prime}\right)\right. \\
& \text { mergulhos isométricos com imagem aberta e causalmente } \\
& \text { convexa }\} ;
\end{aligned}\right.
$$

(Glh é, portanto, uma subcategoria plena de $\mathscr{S} t c)$ e

$$
\mathscr{A} l g b=\left\{\begin{aligned}
\text { objetos: } & \text { Obj } \mathscr{A} l g=C^{*} \text {-álgebras unitais } \mathfrak{A} ; \\
\text { morfismos: } & \text { Hom } \mathscr{A} l g\left(\mathfrak{A}, \mathfrak{A}^{\prime}\right)=\left\{\alpha: \mathfrak{A} \rightarrow \mathfrak{A}^{\prime}{ }^{*}\right. \text {-morfismos unitais } \\
& (\text { não necessariamente injetivos })\} .
\end{aligned}\right.
$$

Uma teoria quântica localmente covariante estendida é um funtor covariante $\mathfrak{A}$ de $\mathscr{S} t_{c_{d}}$ em $\mathscr{A}$ lgb, tal que $\mathfrak{A}_{\Upsilon_{G l h}}$ é uma teoria quântica localmente covariante no sentido

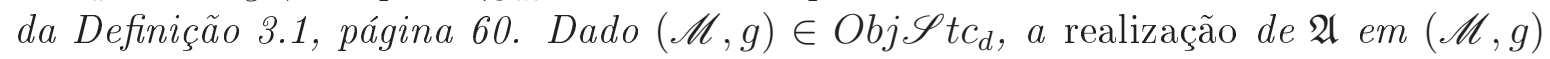
é dada pelo pré-cofeixe $\mathscr{K}(\mathscr{M}, g) \ni \mathscr{O} \mapsto \mathfrak{A}(\mathscr{O}) \doteq \overline{\left(\left\{(A, \mathscr{O}): A \in \mathfrak{A}\left(\mathscr{O}, g\left\lceil_{\mathscr{O}}\right)\right\} / \overline{\mathfrak{I}}_{\mathscr{M}}\right)^{\|\cdot\|}\right.}$, seguindo a construção de BlACKADAR dada acima. Dizemos ainda que $\mathfrak{A}$ é regular se cada álgebra local $\mathfrak{A}(\mathscr{M}, g)$ coincide com a álgebra quase-local da realização de $\mathfrak{A}$ em $(\mathscr{M}, g)$, dada por $(4.3){ }^{4}$

É imediato ver que a realização de $\mathfrak{A}$ em $(\mathscr{M}, g) \in O b j \mathscr{G} l h_{d}$ no sentido da Definição 4.1 coincide com a Definição 3.2 a menos de um *-isomorfismo. A perda da injetividade dos *-morfismos induzidos por $\mathfrak{A}$ se traduz na liberdade de escolha de condições de contorno. Antes de precisar melhor esta idéia, vamos mostrar como ficam as demais propriedades de $\mathfrak{A}$.

\footnotetext{
${ }^{4}$ Aqui, permitimo-nos um ligeiro abuso de notação, uma vez que só lidaremos com o caso regular no que se segue.
} 
- Se $(\mathscr{M}, g)$ possui um grupo não-trivial de isometrias $G \ni \psi$, fica claro que $\psi$ induz uma bijeção de $\mathscr{K}(\mathscr{M}, g) \ni \mathscr{O}$ em si mesmo. Assim, vemos que o *-morfismo $\mathfrak{A} \psi$ é explicitamente inversível, com inversa dada por $\mathfrak{A}\left(\psi^{-1}\right)$, para todo $\psi \in G$. Assim, o Princípio de Covariância Local permite implementar a ação de grupos de isometrias mesmo no caso não globalmente hiperbólico.

- Causalidade local vale no seguinte sentido: se a restrição de $\mathfrak{A}$ à categoria de espaços-tempos globalmente hiperbólicos for localmente causal, e dados, como no exemplo acima, $\mathscr{O}_{1}, \mathscr{O}_{2} \in \mathscr{K}\left(\mathscr{M}_{1}, g_{1}\right)$ causalmente disjuntos com respeito a $\mathscr{O}_{3} \in \mathscr{K}\left(\mathscr{M}_{1}, g_{1}\right)$ (i.e., $\mathscr{O}_{3} \supset \mathscr{O}_{1}, \mathscr{O}_{2}$ e $\left.\mathscr{O}_{1} \perp_{\mathscr{O}_{3}} \mathscr{O}_{2}\right)$, segue por covariância local que

$$
\left[\mathfrak{A} i_{\mathscr{O}_{1}, \mathscr{M}}\left(A_{1}\right), \mathfrak{A} i_{\mathscr{O}_{2}, \mathscr{M}}\left(A_{2}\right)\right]=0,
$$

para todo $A_{i} \in \mathfrak{A}\left(\mathscr{O}_{i}, g\left\lceil_{\mathscr{O}_{i}}\right), i=1,2\right.$.

- Aditividade permanece válida sem alterações, contanto que o recobrimento consista de regiões globalmente hiperbólicas.

- Causalidade primitiva não possui nenhuma extensão imediata para espaços-tempos não globalmente hiperbólicos. No caso de espaços-tempos estavelmente causais, uma definição tentativa pode ser a seguinte: seja $\tau$ uma função tempo global de $(\mathscr{M}, g)$, e $\mathscr{N} \subset \mathscr{M}$ uma vizinhança aberta e causalmente convexa de, digamos, $\Sigma=\tau^{-1}(0)$. Então, dizemos que $\mathfrak{A}$ é fracamente primitivamente causal se for primitivamente causal com respeito a regiões globalmente hiperbólicas e $\mathfrak{A} i_{\mathscr{N}, \mathscr{M}}$ for um *-epimorfismo. Tal definição é conveniente, pois a ambigüidade na escolha de condições de contorno passa a ser expressa de maneira puramente algébrica, da seguinte forma: considere qualquer *-ideal $\mathfrak{J}$ de $\mathfrak{A}\left(\mathscr{N}, g \uparrow_{\mathscr{N}}\right)$ contendo $\operatorname{Ker} \mathfrak{A} \psi$. Se $\mathfrak{A}$ for fracamente primitivamente causal, então a aplicação $A+\mathfrak{J} \mapsto \mathfrak{A} \psi(A)+\mathfrak{A} i_{\mathscr{N}, \mathscr{M}}(\mathfrak{J})$ é um ${ }^{*}$-isomorfismo de $\mathfrak{A}(\mathscr{N}, g\lceil\mathscr{N}) / \mathfrak{J}$ em $\mathfrak{A}(\mathscr{M}, g) / \mathfrak{A} i_{\mathscr{N}, \mathscr{M}}(\mathfrak{J})$, pelo Primeiro Teorema de ${ }^{*}$-Isomorfismo.

A especificação do ideal $\mathfrak{J}$ no contexto de causalidade primitiva fraca corresponde precisamente à escolha de condições de contorno. Que uma escolha "minimal" sempre é possível, é garantido pela definição de causalidade primitiva fraca (basta tomar $\left.\mathfrak{J}=\operatorname{Ker} \mathfrak{A} i_{\mathscr{N}, \mathscr{M}}\right)$. Uma escolha "maximal" seria o caso em que $\mathfrak{J}$ é um ideal maximal de $\mathfrak{A}\left(\mathscr{N}, g\lceil\mathscr{N})\right.$ (a existência de ${ }^{*}$-ideais maximais segue do Lema de ZoRN; ver o Apêndice $\mathrm{C}$ para um enunciado em contexto categórico) - então, a *álgebra quociente $\mathfrak{A}\left(\mathscr{N}, g \uparrow_{\mathscr{N}}\right) / \mathfrak{J}$ é simples, i.e., não possui *-ideais não-triviais, e qualquer *-epimorfismo com domínio em $\mathfrak{A}\left(\mathscr{N}, g\left\lceil_{\mathscr{N}}\right) / \mathfrak{J}\right.$ é automaticamente um *-isomorfismo.

É importante escolher ${ }^{*}$-ideais de contorno $\mathfrak{J}$ que sejam preservados por $\{\mathfrak{A} \psi: \psi \in$ $G(\mathscr{M}, g)\}$, pois só neste caso $G(\mathscr{M}, g)$ é implementado em $\mathfrak{A}(\mathscr{M}, g) / \mathfrak{A} i_{\mathscr{N}, \mathscr{M}}(\mathfrak{J})$ por 
um grupo de ${ }^{*}$-automorfismos. A covariância local é preservada pelo quociente, pois $\mathfrak{A} i_{\mathscr{O}, \mathscr{N}}\left(\mathfrak{A}\left(\mathscr{O}, g\left\lceil_{\mathscr{O}}\right)\right) /\left(\mathfrak{J} \cap \mathfrak{A} i_{\mathscr{O}, \mathscr{N}}\left(\mathfrak{A}\left(\mathscr{O}, g \uparrow_{\mathscr{O}}\right)\right)\right) \cong\left(\mathfrak{A} i_{\mathscr{O}, \mathscr{N}}\left(\mathfrak{A}\left(\mathscr{O}, g\left\lceil_{\mathscr{O}}\right)\right)+\mathfrak{J}\right) / \mathfrak{J}\right.\right.$, pelo Segundo Teorema de *-Isomorfismo (Teorema B.3, página 126).

OBSERVAÇÃo 4.2 Como as construções acima fazem referência unicamente às propriedades de *álgebras, elas se estendem naturalmente para álgebras de BorCHERSUhlmann e, por conseguinte, para campos quânticos localmente covariantes. De fato, no caso de campos livres, a imposição algébrica de condições de contorno engloba o conceito funcional-analítico correspondente no espaço de HILBERT de uma partícula (i.e., extensões auto-adjuntas do gerador Hamiltoniano do operador unitário de evolução temporal) - ver [Som06] para o caso particular da álgebra de WEYL do campo de KLEIN-Gordon em $\left\{x \in \mathbb{R}^{1, d-1}: x^{d-1}>0\right\}$ com condições de contorno de DiRICHLET ou Neumann em $x^{d-1}=0$. Ambos os ${ }^{*}$-ideais neste exemplo são maximais.

\subsection{Teorias quânticas localmente covariantes em AdS}

Vamos agora considerar a realização de uma teoria quântica localmente covariante $\mathfrak{A}$ em $A d S_{d}$ no espírito da Seção 4.1, seguindo o trabalho de BuchHolz, Florig e SumMERS [BFS00]. (A componente conexa à identidade d)o grupo de isometrias de $A d S_{d}$ induz, como visto na Seção 4.1, um grupo de *-automorfismos $\left\{\alpha_{g}: g \in S O_{e}(2, d-1)\right\}$ em $\mathfrak{A}\left(A d S_{d}\right)$ que preserva a estrutura local de $\mathfrak{A}$.

De particular interesse são as álgebras (quase-)locais associadas às cunhas $\mathscr{W}_{p, q}$, $p \ll q \in \mathscr{M} i n(r), r \in \mathscr{I}$. Como visto na Subseção 1.2.2, cada $\mathscr{W}_{p, q}$ possui um grupo uniparamétrico de isometrias $\left\{u_{p, q}^{\lambda}, \lambda \in \mathbb{R}\right\}$ de $A d S_{d}$ que a preservam, dado pela fórmula (1.26). Ressaltamos aqui uma propriedade de $u_{p, q}^{\lambda}$ obtida no contexto mais geral do Capítulo 2 (ver página 50) e que será de grande importância no presente Capítulo: o comportamento assintótico de $g_{p, q}^{\lambda}$ à medida que $\lambda \rightarrow \pm \infty$. Segue imediatamente das fórmulas (1.26) (página 12), (2.45) (página 50) e da ação adjunta de $S O_{e}(2, d-1)$ que, em coordenadas de PoINCARÉ,

$$
u_{p, q}^{\lambda}(x, z) \sim\left\{\begin{array}{ll}
\left(e^{-\lambda}(x-x(q)), e^{-\lambda} z\right) & (\lambda \rightarrow+\infty) \\
\left(e^{\lambda}(x-x(p)), e^{\lambda} z\right) & (\lambda \rightarrow-\infty)
\end{array}, \forall(x, z) \in \mathscr{W}_{p, q}\right.
$$

i.e., $u_{p, q}^{\lambda}$ age assintoticamente como transformações de escala ao redor de $p$ (resp. q) à medida que $\lambda \rightarrow-\infty$ (resp. $\lambda \rightarrow+\infty$ ). Passada esta preliminar geométrica, vamos especificar o que assumimos de nossos modelos físicos:

1. O modelo é dado pela realização de uma teoria quântica localmente covariante estendida regular $\mathfrak{A}$ na coleção $\mathscr{W}\left(A d S_{d}\right)$ das cunhas de $A d S_{d}$, que, por sua vez, 
fornecem por covariância local uma realização de $\mathfrak{A}$ em $A d S_{d}$, conforme a construção da Seção 4.1.

2. Supomos que o grupo $\mathfrak{A} u_{p, q}^{\lambda}$ de *-automorfismos de $\mathfrak{A}\left(\mathscr{W}_{p, q}\right)$ é fortemente contínuo para todo $(p, q) \in \mathscr{D}(\mathscr{I})$.

3. $\mathfrak{A}$ é localmente causal com respeito a regiões globalmente hiperbólicas. ${ }^{5}$

Optamos por realizar $\mathfrak{A}$ não apenas em $A d S_{d}$, mas também em cada cunha individualmente, pois isto não apenas nos permitirá introduzir condições de contorno fisicamente razoáveis como também estender boa parte das considerações que se seguem para espaços-tempos localmente AAdS. A condição sobre os estados "elementares" será discutida na Subseção 4.2.1 a seguir.

Concluímos esta Subseção esboçando, por completeza, a versão original da dualidade de REHREN [Reh00].

DefiniçÃo 4.3 A teoria quântica ReHREN-dual à realização de $\mathfrak{A}$ em AdS $S_{d}$ é o précofeixe conformalmente covariante $\mathscr{D}_{p, q} \mapsto \mathfrak{A}\left(\mathscr{D}_{p, q}\right) \doteq \mathfrak{A}\left(\rho_{A d S_{d}}^{-1}\left(\mathscr{D}_{p, q}\right)\right)=\mathfrak{A}\left(\mathscr{W}_{p, q}\right)$ de $C^{*}$-álgebras, indexado pela coleção de diamantes em $\mathscr{I}$. Aqui, $\rho_{A d S_{d}}$ é a bijeção de REHREN (1.32) (página 16).

\subsubsection{Valores de fronteira como condição dinâmica}

É natural entender cada $\mathfrak{A}\left(\mathscr{W}_{p, q}\right)$ como um sistema $\mathrm{C}^{*}$-dinâmico, cujo grupo de *-automorfismos é dado por $\mathfrak{A} u_{p, q}^{\lambda}$. Os estados "elementares" $\omega$ em $\mathfrak{A}\left(A d S_{d}\right)$ devem representar uma situação de equilíbrio termodinâmico, uma vez que a dinâmica é *automorfa - fisicamente, o sistema dinâmico quântico $\left(\mathfrak{A}\left(\mathscr{W}_{p, q}\right), \mathfrak{A} u_{p, q}\right)$ é fechado.

Exigimos que, para qualquer observador uniformemente acelerado associado a $\mathfrak{A} u_{p, q}^{\lambda}$, um estado $\omega$ sobre $\mathfrak{A}\left(\mathscr{W}_{p, q}\right)$ seja passivo, i.e.,

$$
-i \omega\left(U^{*} \delta_{p, q} U\right) \geq 0
$$

para todo $U \in \mathfrak{U}_{1}\left(\mathfrak{A}\left(\mathscr{W}_{p, q}\right)\right) \cap D\left(\delta_{p, q}\right)$, onde $\delta_{p, q}$ é a derivação que gera $\mathfrak{A} u_{p, q}^{\lambda}$, com domínio $D\left(\delta_{p, q}\right) \subset \mathfrak{A}\left(\mathscr{W}_{p, q}\right)$, e $\mathfrak{U}_{1}\left(\mathfrak{A}\left(\mathscr{W}_{p, q}\right)\right)$ é a componente conexa à identidade do grupo de elementos unitários de $\mathfrak{A}\left(\mathscr{W}_{p, q}\right)$. O requerimento de passividade significa que não é possível extrair energia de $\omega$ por um processo cíclico - por isso, entende-se perturbações internas ao sistema dependentes do tempo que são desligadas fora de um intervalo finito de tempo. Equivalentemente, $\omega$ satisfaz a segunda lei da termodinâmica na formulação de KELVin (em particular, $\omega$ é invariante por $\mathfrak{A} u_{p, q}^{\lambda}$, garantindo a implementabilidade

\footnotetext{
${ }^{5}$ Esta condição não é assumida em [BFS00], que emprega uma adaptação minimal do formalismo de HAAG e KaStler para espaços-tempos AdS.
} 
unitária de $\mathfrak{A} u_{p, q}^{\lambda}$ no espaço de HiLBerT GNS $\mathscr{H}_{\omega}$ ), e o primeiro membro de (4.6) corresponde à entropia (relativa) do estado $\omega\left(U^{*} . U\right)$ em relação ao estado $\omega$ (ver Apêndice $\mathrm{B}$, página 138). Requeremos ainda que $\omega$ satisfaça ergodicidade fraca (em média):

$$
\lim _{T \rightarrow+\infty} \frac{1}{T} \int_{0}^{T} \omega\left(A \mathfrak{A} u_{p, q}^{\lambda}(B)\right) d \lambda=\omega(A) \omega(B), \forall A, B,
$$

significando que $\omega$ é um estado invariante extremal, ou seja, todo parâmetro de ordem (elemento do comutante da representação GNS de $\mathfrak{A}$ associada a $\omega$ invariante pela ação de $\left.\mathfrak{A} u_{p, q}^{\lambda}\right)$ é um "c-number". Nesse caso, segue que $\omega$ é um estado KMS para alguma temperatura inversa $\beta \in[0,+\infty]$, i.e., para toda $f \in \mathscr{S}(\mathbb{R}) \operatorname{com} \hat{f} \in \mathscr{C}_{c}^{\infty}(\mathbb{R})$, segue que

$$
\int_{-\infty}^{+\infty} \omega\left(A \mathfrak{A} u_{p, q}^{\lambda}(B)\right) f(\lambda) d \lambda=\int_{-\infty}^{+\infty} \omega\left(\mathfrak{A} u_{p, q}^{\lambda}(B) A\right) f(\lambda+i \beta) d \lambda, \forall A, B .
$$

O caso $\beta=+\infty$, correspondendo ao caso em que $\omega$ é um estado fundamental, é excluído pela seguinte consideração: considerando, por exemplo, a cunha $\mathscr{W}=\{X$ : $\left.\eta(X, X)=-1, X^{1}>\left|X^{0}\right|, X^{d}>0\right\}$ no domínio fundamental (1.5) de $A d S_{d}$ (página 5), temos que o gerador do subgrupo de isotropia de $\mathscr{W}$ é o gerador $M_{01}$ de $S O_{e}(2, d-1)$. Como $e^{-i \pi M_{12}} M_{01} e^{-i \pi M_{12}}=-M_{01}$, segue da invariância do espectro por transformações unitárias [RS80] que o operador Hamiltoniano no espaço de HiLBERT GNS $\mathscr{H}_{\omega}$ implementando $M_{01}$ não pode ser um operador positivo - o mesmo vale, portanto, para todas as cunhas. BuchHolz, Florig E Summers [BFS00] mostraram que todo $\omega$ que satisfaz (4.6)-(4.7) apresenta o efeito UnRUH, i.e., é um estado KMS $\operatorname{com} \beta=\frac{1}{k T}=\frac{2 \pi}{\kappa}=2 \pi$, onde $\kappa=1$ é a gravidade de superfície em $\partial_{ \pm} \mathscr{W}_{p, q}$ (Teorema 2.6, página 53). Mais ainda, obtemos que a realização de $\mathfrak{A}$ em $A d S_{d}$ é $\omega$-fracamente localmente causal com respeito a $\mathscr{W}\left(A d S_{d}\right): \operatorname{dados} A \in \mathfrak{A}\left(\mathscr{W}_{p, q}\right)$ e $B \in \mathfrak{A}\left(\mathscr{W}_{p^{\prime}, q^{\prime}}\right), \mathscr{W}_{p^{\prime}, q^{\prime}} \subset \mathscr{W}_{p, q}^{\prime}$, temos que $\omega(A B)=\omega(B A)$, mesmo se não exigirmos causalidade local de $\mathfrak{A}$ em forma alguma.

Vamos agora estabelecer um sentido no qual as condições (4.6)-(4.7) correspondem a uma escolha de condições de contorno no infinito conforme. Se um estado $\omega$ em $\mathfrak{A}\left(\mathscr{W}_{p, q}\right)$ é passivo (4.6) e fracamente ergódico em média (4.7) com respeito ao grupo de *-automorfismos $\mathfrak{A} u_{p, q}^{\lambda}$, então o vetor cíclico $\Omega$ no espaço de HilberT GNS $\mathscr{H}_{\omega}$ associado a $\omega$ é separante com respeito a $\pi_{\omega}\left(\mathfrak{A}\left(\mathscr{W}_{p, q}\right)\right)^{\prime \prime}$, em virtude da condição KMS, e $\mathfrak{A} u_{p, q}^{\lambda}$ estende-se a um grupo de ${ }^{*}$-automorfismos de $\pi_{\omega}\left(\mathfrak{A}\left(\mathscr{W}_{p, q}\right)\right)^{\prime \prime}$ que coincide com o grupo modular de TOMITA-TAKESAKI $\sigma_{\omega}^{(2 \pi)^{-1} \lambda}$ (ver Apêndice B, em particular o Lema B.18, página 143, e o Teorema B.19, página 144). Uma vez que assumimos passividade e ergodicidade fraca em média de um estado "elementar" $\omega$ em $\mathfrak{A}_{A d S_{d}}$ com respeito a todos os sistemas $\mathrm{C}^{*}$-dinâmicos $\left(\mathfrak{A}\left(\mathscr{W}_{p, q}\right), \mathfrak{A} u_{p, q}^{\lambda}\right), p \ll q \in \mathscr{I}$, segue que tal $\omega$ satisfaz:

- A propriedade de REEH-Schlieder com respeito à coleção de cunhas $\mathscr{W}\left(A d S_{d}\right)$, i.e., $\omega$ é cíclico e separante com respeito às subálgebras de VON NEUMANN 


$$
\pi_{\omega}\left(\mathfrak{A}\left(\mathscr{W}_{p, q}\right)\right)^{\prime \prime}{ }^{6} \mathrm{e}
$$

- A ação de $\mathfrak{A} u_{p, q}^{\lambda}$ em $\pi_{\omega}\left(\mathfrak{A}\left(\mathscr{W}_{p, q}\right)\right)^{\prime \prime}$ é dada precisamente pelo grupo modular de TOMITA-TAKESAKI $\sigma_{u_{\omega}\left(\mathfrak{A}\left(\mathscr{W}_{p, q}\right)\right)^{\prime \prime}}^{(2 \pi)^{-1} \lambda}$.

Denotaremos por $\Delta_{p, q}$ o operador modular que implementa $\mathfrak{A} u_{p, q}^{\lambda}$, i.e., $\pi_{\omega}\left(\mathfrak{A} u_{p, q}^{2 \pi \lambda}(A)\right)=$ $\Delta_{p, q}^{-i \lambda} \pi_{\omega}(A) \Delta_{p, q}^{i \lambda}$ para todo $A \in \mathfrak{A}\left(\mathscr{W}_{p, q}\right)$. Consideremos agora a inclusão de álgebras de VON Neumann $\pi_{\omega}\left(\mathfrak{A}\left(\mathscr{W}_{r, q}\right)\right)^{\prime \prime} \subset \pi_{\omega}\left(\mathfrak{A}\left(\mathscr{W}_{p, q}\right)\right)^{\prime \prime}$, onde $p \ll \mathscr{I} r \ll \mathscr{g} q$ e, portanto, $\mathscr{W}_{r, q} \subset \mathscr{W}_{p, q}$ e $\partial_{+} \mathscr{W}_{r, q} \subset \partial_{+} \mathscr{W}_{p, q}$. A inclusão das respectivas $\mathrm{C}^{*}$-álgebras subjacentes satisfaz as seguintes propriedades, em virtude da regularidade e da covariância local de $\mathfrak{A}:$

1. $\cup_{\lambda \in \mathbb{R}} \mathfrak{A} u_{p, q}^{\lambda}\left(\mathfrak{A}\left(\mathscr{W}_{r, q}\right)\right)$ é $\mathrm{C}^{*}$-densa em $\mathfrak{A}\left(\mathscr{W}_{p, q}\right)$;

2. $\mathfrak{A} u_{p, q}^{\lambda}\left(\mathfrak{A}\left(\mathscr{W}_{r, q}\right)\right) \subset \mathfrak{A}\left(\mathscr{W}_{r, q}\right)$ para todo $\lambda \geq 0$.

Segue de 1.) e 2.) que a inclusão $\pi_{\omega}\left(\mathfrak{A}\left(\mathscr{W}_{r, q}\right)\right)^{\prime \prime} \subset \pi_{\omega}\left(\mathfrak{A}\left(\mathscr{W}_{p, q}\right)\right)^{\prime \prime}$ satisfaz as condições propostas no trabalho de BORCHERS e YNGVASON [BY99]. Os resultados principais deste trabalho, refraseados para nosso presente contexto, são:

TeOrema 4.1 ([BY99], TeOREma 2.1) Defina $\mathfrak{M} \doteq \pi_{\omega}\left(\mathfrak{A}\left(\mathscr{W}_{p, q}\right)\right)^{\prime \prime}, \mathfrak{N} \doteq \pi_{\omega}\left(\mathfrak{A}\left(\mathscr{W}_{r, q}\right)\right)^{\prime \prime}$, $\Delta_{p, q}^{i \lambda} \doteq T(-2 \pi \lambda)$ e $\mathfrak{N}(\lambda)=\operatorname{Ad} T(\lambda) \mathfrak{N} \doteq T(\lambda) \mathfrak{N} T(-\lambda)$. Neste caso,

(i) $\operatorname{Ad} \Delta_{r, q}^{i u} \mathfrak{N}(\lambda)=\mathfrak{N}(\varphi(u, \lambda))$, onde

$$
\varphi(u, \lambda)=\log \left(1+e^{-2 \pi u}\left(e^{\lambda}-1\right)\right)
$$

para todo $(u, \lambda) \in \mathbb{R}^{2}$ para o qual o lado direito é definido. Em particular, $\operatorname{Ad} \Delta_{r, q}^{i u} \mathfrak{M} \subset \mathfrak{M}$ para todo $u \geq 0$, e $\mathfrak{N}=\cap_{u \geq 0} \operatorname{Ad} \Delta_{r, q}^{i u} \mathfrak{M}$

(ii) O operador $G \doteq \log \Delta_{p, q}-\log \Delta_{r, q}$ é não-negativo e essencialmente auto-adjunto num "core" comum a $\log \Delta_{p, q}$ e $\log \Delta_{r, q} \cdot{ }^{7}$ O grupo unitário uniparamétrico $\Gamma(\tau) \doteq$ $e^{(2 \pi)^{-1} i \tau \bar{G}}$ satisfaz $T(\lambda) \Gamma(\tau) T(\lambda)=\Gamma\left(e^{\lambda} \tau\right)$ e $\operatorname{Ad} \Gamma(\tau) \mathfrak{N}(\lambda)=\mathfrak{N}(\psi(\tau, \lambda))$, onde

$$
\psi(\tau, \lambda)=\lambda+\log \left(1+\tau e^{-\lambda}\right)
$$

para todo $(\tau, \lambda) \in \mathbb{R}^{2}$ para o qual o lado direito é definido. Em particular, $\operatorname{Ad} \Gamma(\tau) \mathfrak{M} \subset \mathfrak{M}$ e $\operatorname{Ad} \Gamma(\tau) \mathfrak{N} \subset \mathfrak{N}$ para todo $\tau \geq 0$, e $\mathfrak{N}=\operatorname{Ad} \Gamma(1) \mathfrak{M}$.

\footnotetext{
${ }^{6}$ Essa propriedade é conhecida pelos especialistas em Teoria de Campos Conforme como "correspondência operador-estado".

${ }^{7}$ Um "core" comum aqui é um subconjunto linear $D$ denso em $\mathscr{H}_{\omega}$ tal que $D \subset D\left(\log \Delta_{p, q}\right) \cap$ $D\left(\log \Delta_{r, q}\right)$ e tal que $\overline{\log \Delta_{p, q}\left\lceil_{D}\right.}=\log \Delta_{p, q}$ e $\overline{\log \Delta_{r, q}\left\lceil_{D}\right.}=\log \Delta_{r, q}$, onde $D\left(\log \Delta_{p, q}\right), D\left(\log \Delta_{r, q}\right) \subset \mathscr{H}_{\omega}$ são respectivamente os domínios dos operadores $\log \Delta_{p, q}$ e $\log \Delta_{r, q}$.
} 
Em suma, o Teorema 4.1 não só dá uma forma precisa para as relações de comutação entre $\Delta_{p, q}^{i \lambda}$ e $\Delta_{r, q}^{i u}$, como também fornece uma realização geométrica para a ação de $u \mapsto \operatorname{Ad} \Delta_{r, q}^{i u}$ a partir da de $\lambda \mapsto \operatorname{Ad} \Delta_{p, q}^{i \lambda}$ sem que tenhamos que assumir a existência da primeira. Os próximos dois Teoremas mostram que as ações destes grupos unitários tornam-se essencialmente indistinguíveis se estamos geometricamente suficientemente longe de $\partial_{-} \mathscr{W}_{r, q}$ :

Teorema 4.2 ([BY99], TeOrema 2.2) Empregando a notação do Teorema 4.1, se $A \in \mathfrak{N}(\lambda)$ e $B \in \mathfrak{N}^{\prime}$, temos que

$$
\begin{gathered}
\left|\left\langle B \Omega, \Delta_{r, q}^{i u} A \Omega\right\rangle-\langle B \Omega, T(-2 \pi u) A \Omega\rangle\right| \leq 2 \max \left\{\|A \Omega\|\|B \Omega\|,\left\|A^{*} \Omega\right\|\left\|B^{*} \Omega\right\|\right\} . \\
. \min \left\{\frac{\left|2 e^{2 \pi u}-1\right|}{e^{\lambda}-1}, 1\right\}
\end{gathered}
$$

para todo $\lambda>0, u \in \mathbb{R}$.

Teorema 4.3 ([BY99], TeOrema 2.3) Empregando a notação do Teorema 4.1, temos que:

(i)

$$
\lim _{\lambda \rightarrow+\infty}\left\|\Delta_{r, q}^{i u}(\operatorname{Ad} T(\lambda) A) \Psi-T(-2 \pi u)(\operatorname{Ad} T(\lambda) A) \Psi\right\|=0
$$

para todo $A \in \mathfrak{M}$ e $\Psi \in \mathscr{H}_{\omega}$, com convergência uniforme em $u \in\left(-\infty, u_{0}\right]$ para todo $u_{0}<+\infty$;

(ii)

$$
\lim _{\lambda \rightarrow+\infty}\left\|\Delta_{r, q}^{i u} \operatorname{Ad} T(\lambda) A-T(-2 \pi u) \operatorname{Ad} T(\lambda) A\right\|=0
$$

para todo A numa subálgebra fortemente densa em $\mathfrak{M}$, com convergência uniforme em $u \in\left(-\infty, u_{0}\right]$ para todo $u_{0}<+\infty$.

Em suma, denotando $\mathscr{D}_{p, q}=I^{-}\left(q, \mathscr{M} i n(p) \cong \mathbb{R}^{1, d-2}\right)$, segue que tais grupos são essencialmente indistinguíveis das dilatações por um fator $e^{-2 \pi \lambda}$ ao redor de $q$ para $\lambda \rightarrow+\infty$. Levando em consideração a ação do subgrupo de dilatações de $\mathscr{R}^{1, d-2}$ em $A d S_{d}$, obtemos que os grupos modulares associados a $\omega$ e às cunhas em $A d S_{d}$ implementam assintoticamente transformações de escala ao redor de pontos de $\mathscr{I}$, no sentido preciso dado pelos Teoremas 4.2 e 4.3 - todas as considerações acima permanecem válidas se invertemos a orientação temporal, i.e., trocando os papéis de $p$ e $q$.

Mais em geral, inspirando-nos no formalismo proposto em [BV95], escrevemos a seguinte 
DEFiniÇÃo 4.4 Sejam $p \ll q \in \mathscr{M}$ in $(r)$ para algum $r \in \mathscr{I}$, onde assume-se que $\mathscr{M}$ in $(r)$ é dotado das operações de espaço vetorial de $\mathbb{R}^{1, d-2}$. A álgebra de escala futura $\mathfrak{A}^{+}\left(\mathscr{W}_{p, q}\right)$ (resp. passada $\mathfrak{A}^{-}\left(\mathscr{W}_{p, q}\right)$ ) em $\mathscr{W}_{p, q}$ consiste das funções uniformemente limitadas em norma $(0,1] \ni \theta \mapsto \underline{A}^{ \pm}(\theta) \doteq A_{\theta}^{ \pm} \in \mathfrak{A}\left(\mathscr{W}_{p, q}\right)$ satisfazendo $A_{1}^{+} \doteq$ $A^{+} \in \mathfrak{A}\left(\mathscr{W}_{r, s}\right) \Rightarrow A_{\theta}^{+} \in \mathfrak{A}\left(\mathscr{W}_{\theta r+(1-\theta) q, \theta s+(1-\theta) q}\right)$ (resp. $A_{1}^{-} \doteq A^{-} \in \mathfrak{A}\left(\mathscr{W}_{r, s}\right) \Rightarrow A_{\theta}^{-} \in$ $\left.\mathfrak{A}\left(\mathscr{W}_{\theta r+(1-\theta) p, \theta s+(1-\theta) p}\right)\right)$, para todo $p \leq \mathscr{I} r \ll \mathscr{I} s \leq \mathscr{I} q, \theta \in(0,1]$. As operações algébricas são definidas ponto a ponto, e a $C^{*}$-norma é dada por $\left\|\underline{A}^{ \pm}\right\| \doteq \sup _{0<\theta \leq 1}\left\|A_{\theta}^{ \pm}\right\|$.

OBSERVAÇÃo 4.5 Podemos dizer que o conceito de álgebras de escala define uma versão abstrata do Grupo de Renormalização em Fúsica Quântica Local. Uma definição diferente, mais rudimentar, para álgebras de escala associadas a cunhas em $A d S_{d}$ foi proposta pelo Autor em [Rib04].

Note que a definição acima preserva isotonia e causalidade local das subálgebras locais de $\mathfrak{A}\left(\mathscr{W}_{p, q}\right)$.

Dado um estado $\omega$ em $\mathfrak{A}\left(\mathscr{W}_{p, q}\right)$, podemos efetuar seu levantamento ("lift") $\omega_{\theta}^{ \pm}$a $\mathfrak{A}^{ \pm}\left(\mathscr{W}_{p, q}\right)$ na escala $\theta \in(0,1]$ definindo $\omega_{\theta}^{+}\left(\underline{A}^{+}\right) \doteq \omega\left(A_{\theta}^{+}\right)$e $\omega_{\theta}^{-}\left(\underline{A}^{-}\right) \doteq \omega\left(A_{\theta}^{-}\right)$. Obviamente, $\omega_{\theta}^{+}$define um estado em $\mathfrak{A}^{ \pm}\left(\mathscr{W}_{p, q}\right)$.

Consideremos $s=q$ na Definição 4.4, i.e., temos a situação de inclusão considerada nos Teoremas 4.1 a 4.3. Neste caso, temos que $\theta \mapsto A_{\theta}^{+} \doteq \mathfrak{A} u_{p, q}^{f_{r,+}^{-1}(\theta)}(A)$, onde $A \in$ $\mathfrak{A}\left(\mathscr{W}_{r, q}\right)$ e

$$
f_{r,+}(\lambda)=\frac{d_{\bar{g}^{(0)}}\left(u_{p, q}^{\lambda}(r), q\right)}{d_{\bar{g}^{(0)}}(r, q)}, f_{r,-}(\lambda)=\frac{d_{\bar{g}^{(0)}}\left(p, u_{p, q}^{-\lambda}(r)\right)}{d_{\bar{g}^{(0)}}(p, r)}
$$

são funções bijetoras, $\mathscr{C}^{\infty}$ e estritamente decrescentes de $[0,+\infty) \ni \lambda$ em $(0,1] \ni \theta$, define um elemento de $\mathfrak{A}^{+}\left(\mathscr{W}_{r, q}\right)$ e, portanto, de $\mathfrak{A}^{+}\left(\mathscr{W}_{p, q}\right)$. Conversamente, podemos definir uma família de *-automorfismos $\delta_{p, q}^{\mu,+}$ de $\mathfrak{A}^{+}\left(\mathscr{W}_{p, q}\right)$ implementando transformações de escala ao redor de $q$ por um fator $\mu \in(0,1]$ através da fórmula $\left(\delta_{p, q}^{\mu,+} \underline{A}^{+}\right)(\theta) \doteq A_{\mu \theta}^{+}$. Levando em consideração a relação de comutação

$$
\theta u_{p, q}^{\lambda}(r)+(1-\theta) q=u_{\theta p+(1-\theta) q, q}^{\theta \lambda}(\theta r+(1-\theta) q)
$$

podemos induzir uma ação de $\mathfrak{A} u_{p, q}^{\lambda}$ em $\mathfrak{A}^{+}\left(\mathscr{W}_{p, q}\right)$ através da fórmula

$$
\mathfrak{A}^{+} u_{p, q}^{\lambda}\left(\underline{A}^{+}\right)(\theta) \doteq \mathfrak{A} u_{\theta p+(1-\theta) q, q}^{\theta \lambda}\left(A_{\theta}^{+}\right) .
$$

Considerações análogas valem para as álgebras de escala passadas, com notação de acordo, se invertermos a orientação temporal, trocarmos os papéis de $r$ e $s$ e substituir$\operatorname{mos} f_{r,+}$ por $f_{s,-}$. Assumimos que a ação $(4.10)$ e seu análogo passado são fortemente 
contínuas respectivamente em $\mathfrak{A}^{+}\left(\mathscr{W}_{p, q}\right)$ e $\mathfrak{A}^{-}\left(\mathscr{W}_{p, q}\right)$.

A razão de ser da descrição redundante da realização de $\mathfrak{A}$ em $A d S_{d}$ feita pelas álgebras de escala passadas e futuras vem do fato de que podemos, com ela, definir limites de escala: notemos que a rede de estados $\left\{\omega_{\theta}^{+}: \theta \in(0,1]\right\}$ induzidos por $\omega$ sempre possui subredes pontualmente convergentes (topologia ${ }^{*}$-fraca no dual de $\mathfrak{A}^{+}\left(\mathscr{W}_{p, q}\right)$ ) a estados $\omega_{0, \iota}^{+}$em $\mathfrak{A}^{+}\left(\mathscr{W}_{p, q}\right)$ ( $I \ni \iota$ é algum conjunto de índices), pelo teorema de BANACHAlaOGLU [RS80]. A representação GNS $\pi_{0, \iota}^{+}$associada a cada $\omega_{0, \iota}^{+}$dos elementos de $\mathfrak{A}^{+}\left(\mathscr{W}_{p, q}\right)$ têm, assim, a interpretação de limites (fracos) de escala dos procedimentos locais. Entretanto, resta a questão da unicidade das representações $\pi_{0, \iota}^{+}$a menos de isomorfismos de rede, i.e., da existência ou não de ${ }^{*}$-isomorfismos $\phi_{\iota, \iota^{\prime}}$ de $\pi_{0, \iota}^{+}\left(\mathfrak{A}^{+}\left(\mathscr{W}_{p, q}\right)\right)$ em $\pi_{0, \iota^{\prime}}^{+}\left(\mathfrak{A}^{+}\left(\mathscr{W}_{p, q}\right)\right)$ que preservem a estrutura local $-\phi_{\iota, \iota^{\prime}}\left(\pi_{0, \iota}^{+}\left(\mathfrak{A}^{+}\left(\mathscr{W}_{r, s}\right)\right)\right)=\pi_{0, \iota^{\prime}}^{+}\left(\mathfrak{A}^{+}\left(\mathscr{W}_{r, s}\right)\right)$ para todo $p \leq_{\mathscr{I}} r \ll \mathscr{I} s \leq_{\mathscr{I}} q, \iota, \iota^{\prime} \in I$. Tal questão é parcialmente elucidada pela seguinte

Proposição 4.4 Suponha que $\omega$ é um estado em $\mathfrak{A}_{A d S_{d}}$ passivo e fracamente ergódico em média com respeito aos sistemas $C^{*}$-dinâmicos $\left(\mathfrak{A}\left(\mathscr{W}_{p, q}\right), \mathfrak{A} u_{p, q}^{\lambda}\right)$, para todo par $(p, q) \in \mathscr{D}(\mathscr{I})$. Então,

(i) O estado limite $\omega_{0, \iota}^{+}$é $\beta$-KMS com $\beta=2 \pi$ em relação ao sistema dinâmico $\left(\pi_{0, \iota}^{+}\left(\mathfrak{A}^{+}\left(\mathscr{W}_{p, q}\right)\right), \mathfrak{A}_{0, \iota}^{+, \iota} u_{p, q}^{\lambda}\right)$, onde $\mathfrak{A}_{0, \iota}^{+} u_{p, q}^{\lambda}\left(\pi_{0, \iota}^{+}(A)\right) \doteq \pi_{0, \iota}^{+}\left(\mathfrak{A}^{+} u_{p, q}^{\lambda}(A)\right)$.

(ii) Os limites de escala $\pi_{0, \iota}^{+}\left(\mathfrak{A}^{+}\left(\mathscr{W}_{r, s}\right)\right)$ e $\pi_{0, \iota}^{+}\left(\mathfrak{A}^{+}\left(u_{p, q}^{\lambda}\left(\mathscr{W}_{r, s}\right)\right)\right)$ são unitariamente equivalentes, para todo $p \leq_{\mathscr{I}} r \ll \mathscr{I} s \leq_{\mathscr{I}} q$.

(iii) Se $\omega$ for primário, i.e. $\pi_{\omega}\left(\mathfrak{A}_{A d S_{d}}\right)$ " é um fator (ver Apêndice B, página 132), satisfaz então a seguinte propriedade de definiteza local holográfica: dado $r \in \mathscr{I}$, temos que

$$
\bigcap_{\substack{(p, q) \in \mathscr{D}(\mathscr{I}), \mathscr{W} p, q \ni r}} \pi_{\omega}\left(\mathfrak{A}\left(\mathscr{W}_{p, q}\right)\right)^{\prime \prime}=\mathbb{C} \mathbb{1} .
$$

Segue desta que os conjuntos de limites de escala $\left\{\left(\omega^{\prime}\right)_{0, \iota^{\prime}}^{+}: \iota^{\prime} \in I^{\prime}\right\}$ de qualquer estado $\omega^{\prime}$ no fólio local de $\omega$ com respeito à coleção de cunhas (i.e., $\omega^{\prime}\left\lceil\mathfrak{A}\left(\mathscr{W}_{p, q}\right)\right.$ define um estado normal em $\pi_{\omega}\left(\mathfrak{A}\left(\mathscr{W}_{p, q}\right)\right)^{\prime \prime}$ para todo $\left.(p, q) \in \mathscr{D}(\mathscr{I})\right)$ coincidem $\operatorname{com}\left\{\omega_{0, \iota}^{+}: \iota \in I\right\}$. Em conseqüência disto e de (i), cada álgebra de VON NEUMANN $\pi_{\omega}\left(\mathfrak{A}\left(\mathscr{W}_{p, q}\right)\right)^{\prime \prime}$, se infinita (o que é geralmente o caso em Teoria Quântica de Campos), é tipo III (ver Apêndice B, Definição B.5, página 135).

(iv) Suponha que a aplicação linear de $\pi_{\omega}\left(\mathfrak{A}\left(\mathscr{W}_{p, q}\right)\right)$ em $\mathscr{H}_{\omega}$ dada por

$$
\pi_{\omega}(A) \mapsto E_{p, q}(K) \pi_{\omega}(A) \Omega,
$$

onde $E_{p, q}$ é a medida de BOREL a valores nas projeções de $\mathscr{H}_{\omega}$ que define a resolução espectral do operador $\log \Delta_{p, q}$ e $K$ é um compacto em $\mathbb{R}=\sigma\left(\log \Delta_{p, q}\right)$, é 
uma aplicação compacta, i.e., leva conjuntos limitados no dominio em conjuntos relativamente compactos na imagem, para todo $(p, q) \in \mathscr{D}(\mathscr{I}) .{ }^{8}$ Então as aplicações $\phi_{\iota}: \pi_{0, \iota}^{+}\left(\underline{A}^{+}\right) \mapsto w-\lim _{\kappa} \pi_{\omega}\left(\mathfrak{A} u_{p, q}^{-f_{p,+}^{-1}\left(\theta_{\kappa}\right)}\left(A_{\theta_{\kappa}}^{+}\right)\right)$, onde $\left\{\omega_{\theta_{\kappa}}^{+}\right\}_{\kappa}$ é uma subrede que converge ${ }^{*}$-fracamente a $\omega_{0, \iota}^{+}$, definem isomorfismos de rede com a representação GNS da rede original associada a $\omega$. Em particular, em virtude de (i), os limites de escala são todos unitariamente equivalentes entre si, e o grupo modular de qualquer limite de escala age assintoticamente como o grupo modular associado $a \omega_{1}^{+}$para $\lambda \rightarrow+\infty$.

\section{Prova (ESBoÇO).}

(i) Segue do Teorema 5.3.30 em [BR97] que o limite *-fraco de uma rede de estados $\beta$-KMS é $\beta$-KMS.

(ii) Segue imediatamente da hipótese de continuidade de $\mathfrak{A}^{+} u_{p, q}^{\lambda}$ a existência de um *-isomorfismo, que é unitariamente implementado em virtude da existência de vetores cíclicos e separantes para ambas as álgebras (ver o Teorema 2.5.32 em [BR87] para uma prova deste último fato).

(iii) A primeira assertiva é obtida empregando-se a dualidade de REHREN: Em virtude de (i) e (ii), o grupo conforme de $\mathscr{M} i n(r) \cong \mathbb{R}^{1, d-2}$ para todo $r \in \mathscr{I}$ é unitariamente implementado em $\mathscr{H}_{\omega}$ pelos grupos modulares de TOMiTATAKESAKI associados a $\mathfrak{A} \circ \rho_{A d S_{d}}^{-1}\left(\mathscr{D}_{p, q}\right)=\mathfrak{A}\left(\mathscr{W}_{p, q}\right)$, e $\omega$ é um estado conformalmente invariante da teoria REHREN-dual $\mathfrak{A} \circ \rho_{A d S_{d}}^{-1}$. Em virtude das relações de comutação resultantes entre os diferentes grupos modulares, segue da Proposição 2.3 em [BS93] que os geradores $P^{\mu}$ das translações em $\mathscr{M}$ in $(r)$ satisfazem a condição espectral, i.e., $\sigma(P) \subset J^{+}\left(0, \mathbb{R}^{1, d-2}\right)$ - em suma, $\omega$ é um vácuo. Como $\omega$ é primário, segue então do Teorema 4.6 em [Ara99] que $\omega$ é puro e que todo elemento de $\mathscr{H}_{\omega}$ invariante por translações é proporcional a $\Omega$. Seja agora um elemento $Z \in \bigcap_{\substack{\left.(p, q) \in \mathscr{D}(\mathscr{F}) \\ \mathbb{W}_{p, q}\right)}} \pi_{\omega}\left(\mathfrak{A}\left(\mathscr{W}_{p, q}\right)\right)^{\prime \prime} \doteq \mathfrak{N}(r)$. Neste caso, $Z^{*} \in \mathfrak{N}(r)$ e, se $\mathbb{R}^{1, d-2} \ni x \alpha_{x}$ é o grupo de *automorfismos que implementa as translações em $\mathscr{M} i n(s) \ni 0=x(r)$, onde $x$ é uma carta Cartesiana em $\mathscr{M}$ in $(s)$, então $\left[Z^{*}, \alpha_{x}(Z)\right]=0$ para todo $x$ tipo espaço e, por continuidade fraca de $\alpha_{x}$, também para $x=0$ e $x$ tipo luz. Considere a função limitada $\mathbb{R} \ni t \mapsto\left\langle\Omega, Z^{*} \alpha_{t e}(Z) \Omega\right\rangle$, onde $e$ é um vetor tipo luz em $\mathbb{R}^{1, d-2}$. A transformada de Fourier desta função possui, em virtude da

\footnotetext{
${ }^{8}$ Como aplicações lineares compactas são caracterizadas por poderem ser aproximadas em norma por aplicações lineares de posto finito com precisão arbitrária, tal condição nos diz que a densidade espectral de excitações locais do estado fundamental não cresce rápido demais com a energia. Tal condição sobre o comportamento do espaço de fase de teorias quânticas de campos foi proposta por HAAG e SwIEcA para o gerador (Hamiltoniano) de translações temporais no espaço-tempo de Minkowski. Seria interessante saber se a condição de compacidade que demos pode ser deduzida, da mesma forma que a condição de compacidade de HAAG-SWIECA, de outras condições estruturais exploradas na literatura de Teoria Quântica de Campos Algébrica, tais como nuclearidade modular [Haa96].
} 
condição espectral, suporte em $\overline{\mathbb{R}}_{+}$. Devido à comutatividade tipo luz estabelecida acima, tal função coincide com a função $\mathbb{R} \ni t \mapsto\left\langle\Omega, \alpha_{t e}(Z) Z^{*} \Omega\right\rangle$, cuja transformada de Fourier possui suporte em $\overline{\mathbb{R}}_{-}$. Segue destarte que a transformada de Fourier da primeira é suportada em $\{0\}$ e, como tal função é limitada, é necessariamente constante. Portanto, se $x \mapsto U(x)$ é a implementação unitária das translações em $\mathscr{H}_{\omega}$, segue que $U(t e) Z \Omega=Z \Omega$ para todo $t \in \mathbb{R}$ e $e$ tipo luz, logo $U(x) Z \Omega=Z \Omega$ para todo $x$. Pela unicidade de $\Omega$, temos portanto que $Z \Omega=\langle\Omega, Z \Omega\rangle \Omega$. Como $\Omega$ é separante para $\pi_{\omega}\left(\mathfrak{A}\left(\mathscr{W}_{p, q}\right)\right)^{\prime \prime}$ para todo $(p, q) \in \mathscr{D}(\mathscr{I})$, temos que $Z=\langle\Omega, Z \Omega\rangle \mathbb{1}$, como afirmamos. A segunda assertiva segue do fato de que, em virtude da definiteza local holográfica, $\lim _{\theta \backslash 0}\left\|\left.\left(\omega-\omega^{\prime}\right)\right|_{\mathfrak{A}\left(\mathscr{W}_{\theta p+(1-\theta) q, q}\right)}\right\|=0$ (isso pode ser demonstrado de maneira análoga à segunda parte da prova do Lema 4.1 em [BV95], cuja primeira parte é essencialmente a demonstração da primeira assertiva de (iii) acima). A terceira assertiva segue do critério de DrIessler (Teorema B.17, página 139), que, de certa forma, foi criado especialmente para a situação em que nos encontramos.

(iv) Demonstra-se de maneira análoga à Proposição 5.1 em [BV95]. A última assertiva segue então dos Teoremas 4.2 e 4.3 .

OBSERVAÇÃo 4.6 Vale o análogo da Proposição 4.4 para álgebras de escala passadas, se invertermos a orientação temporal e substituirmos $f_{p,+}$ por $f_{q,-}$ no item (iv). Mais ainda, há equivalência unitária entre limites de escala passados e futuros em virtude do item (ii) desta Proposição.

OBSERVAÇÃo 4.7 A implementabilidade unitária das transformações conformes no espaço de HILBERT GNS de um vácuo conformalmente invariante pelos grupos modulares de TOMITA-TAKESAKI foi demonstrada anteriormente por BRUneTti, Guido $e$ Longo [BGL93]. Assim, nossa linha de raciocinio pode ser vista como uma versão "holográfica" desse resultado. Mais ainda, segue do Teorema 2.3 (i) em [BGL93] que a *-representação $\pi_{\omega}$ satisfaz dualidade de HAAG em $\mathscr{I}$ e dualidade de HAAG essencial em $\mathscr{M}$ in $(r)$ para todo $r \in \mathscr{I}$ (para a definição e uma explicação da utilidade e sentido da dualidade de HAAG (essencial), ver a Subseção 4.4.1, página 88). Podemos fazer uso do fato de que dualidade de HAAG essencial vale para os limites de escala e refinar a conclusão do item (iii), provando que $\pi_{\omega}\left(\mathfrak{A}\left(\mathscr{W}_{p, q}\right)\right)^{\prime \prime}$ é um fator tipo $I I I_{1}$ se empregarmos um critério diferente para a estrutura desta álgebra [BV95]. Não provamos este resultado mais forte porque não precisamos desta informação mais refinada no que se segue, e o critério de DRIESSLER, além de mais simples, possui uma interpretação bastante natural no nosso contexto.

A equivalência no item (iv) da Proposição 4.4 mostra que, de fato, (4.6) e (4.7) atuam efetivamente como uma condição sobre limites de escala ao redor de pontos em 
$\mathscr{I}$, revelando assim a relação entre esse conjunto de hipóteses e a formulação de BERTOLA ET AL. [BBMS00], baseada em funções de Wightman de $k$ pontos em $A d S_{d}$. As condições de contorno empregadas neste trabalho, similares às propostas por WITTEN [Wit98] para a correspondência AdS/CFT, são dadas precisamente por um limite de escala das funções de $k$ pontos ao redor de $\mathscr{I}$. O importante item (iii) mostra que o limite de escala é, de fato, uma condição sobre o fólio local de $\omega$ e, portanto, sobre os *-ideais Ann $\omega\left\lceil\mathfrak{A}\left(\mathscr{W}_{p, q}\right)\right.$. Tal condição é localmente covariante em virtude de (ii) e do Primeiro Teorema de *-Isomorfismo (Teorema B.2, página 125), definindo assim uma condição de contorno localmente covariante no sentido da Seção 4.1.

Há, todavia, um ponto muito importante a ser ressaltado: no trabalho de BERTOLA ET AL., o limite de escala define a teoria quântica dual na fronteira (equivalentemente, a teoria quântica na fronteira corresponde neste caso à classe de universalidade da teoria quântica no interior mediante o "fluxo de grupo de renormalização" induzido por transformações de escala ao redor de pontos em $\mathscr{I}$ ), o que não ocorre com a dualidade de Rehren, que consiste em transplantar o conjunto que indexa o pré-cofeixe que realiza a teoria quântica localmente covariante $\mathfrak{A}$ em $A d S_{d}$ por meio da bijeção de REHREN. Uma propriedade estrutural que ilustra bem esta diferença é a propriedade do tubo tipo tempo de Borchers: se $\mathfrak{A}$ é aditiva, e existe uma coleção de regiões $\mathscr{O}_{i} \subset \mathscr{M}$ recobrindo uma vizinhança aberta arbitrariamente pequena de um segmento de curva tipo tempo com extremos $r \ll s \in \mathscr{M}$ tais que $I^{+}(r) \cap I^{-}(s)$ é simplesmente conexo, então

$$
\overline{\bigvee_{i} \mathfrak{A}\left(\mathscr{O}_{i}\right)}=\mathfrak{A}\left(I^{+}(r) \cap I^{-}(s)\right)
$$

Ou seja, o germe das álgebras locais ao redor de um segmento de curva tipo tempo e, em particular, de uma subvariedade tipo tempo é *-isomorfo à álgebra local associada ao seu completamento causal (uma prova deste fato para campos quânticos em espaços-tempos real-analíticos pode ser encontrada em [Str00]). Tal resultado mostra que a dualidade de REHREN (Definição 4.3, página 75) concerne realmente os germes das álgebras ao redor de $\mathscr{I}$ (cuja definição exige, de fato, a imposição de algum tipo de condição de contorno como a elaborada acima ${ }^{9}$ ), sendo portanto mais rica que um mero limite de escala. Tal diferença também se manifesta nas propriedades das álgebras locais da teoria quântica dual na fronteira em ambas as abordagens, conforme discutido por DÜtsch e REHREN [DR03, Reh05].

${ }^{9} \mathrm{Na}$ verdade, fomos um pouco abusivos em nossa definição da propriedade do tubo tipo tempo de Borchers. A formulação correta (da qual não faremos uso) envolve as álgebras de VON NEUMANN locais e fechos na topologia fraca, associados a uma representação GNS gerada por um estado $\omega$. Assim, a definição envolve também propriedades de $\omega$, ou, mais precisamente, de seu fólio local. 
O item (iii) da Proposição 4.4 e os Teoremas 4.2 e 4.3 podem, por outro lado, ser entendidos como propriedades de retorno ao equilíbrio, se imaginarmos as isometrias $u_{p, q}^{\lambda}$ em $A d S_{d}$ como evoluções de CAUCHY com condições de contorno em $\mathscr{I}$, à luz dos resultados do Capítulo 2. Assim, temos uma relação estreita (podemos dizer "holográfica") entre limites de escala ao redor de pontos de $\mathscr{I}$ e termalização dos estados "elementares" $\omega$ através da dinâmica gerada por $\mathfrak{A} u_{p, q}^{\lambda}$. No caso de $A d S_{d}$, esse retorno é trivial, pois vimos que $\omega$ é um estado KMS com respeito a essa dinâmica e, ao mesmo tempo, $\omega$ é um estado da teoria REHREN-dual invariante por transformações de escala. Na próxima Seção, faremos uso deste ponto de vista mais geral e considerar estados "elementares" que, em virtude de efeitos gravitacionais não-triviais, retornam, de acordo, "não-trivialmente" ao equilíbrio e, equivalentemente, apresentam invariância de escala apenas assintótica ao redor de $\mathscr{I}$

\subsection{Holografia algébrica em espaços-tempos AAdS}

Chegamos finalmente ao momento de definir as teorias quânticas de interesse para nós em espaços-tempos AAdS $(\mathscr{M}, g)$ satisfazendo as hipóteses do Teorema 1.3, que garante que $I^{+}(p, \overline{\mathscr{M}}) \cap I^{-}(q, \overline{\mathscr{M}}) \cap \mathscr{I}=I^{+}(p, \mathscr{I}) \cap I^{-}(q, \mathscr{I}) \doteq \mathscr{D}_{p, q}$ para todo $(p, q) \in \mathscr{D}(\mathscr{I})$. A parte mais delicada concerne a implementação das isometrias assintóticas associadas a cunhas construídas no Capítulo 2 (páginas 47-52). Todavia, antes disto, escrevamos:

DefiniçÃo 4.8 A teoria quântica ReHREN-dual à realização de $\mathfrak{A}$ em $(\mathscr{M}, g)$ é o précofeixe $\mathscr{D}_{p, q} \mapsto \mathfrak{A}\left(\mathscr{D}_{p, q}\right) \doteq \mathfrak{A}\left(\rho_{(\mathscr{M}, g)}^{-1}\left(\mathscr{D}_{p, q}\right)\right)=\mathfrak{A}\left(\mathscr{W}_{p, q}\right)$ de $C^{*}$-álgebras, indexado pela coleção de diamantes em $\mathscr{I}$. Aqui, $\rho_{(\mathscr{M}, g)}$ é a bijeção de REHREN (1.32), introduzida na página 16.

Consideremos agora uma cunha $\mathscr{W}_{p, q}$ com fecho geodesicamente convexo. Definamos, para $\lambda \in \mathbb{R}, \epsilon>0$ fixo, as regiões

$$
\mathscr{W}_{p, q}^{+}(\lambda) \doteq\left(\lambda_{p, q}^{\bar{g}}\right)^{-1}((\lambda-\epsilon,+\infty)) \cap \mathscr{M}, \mathscr{W}_{p, q}^{-}(\lambda) \doteq\left(\lambda_{p, q}^{\bar{g}}\right)^{-1}((-\infty, \epsilon-\lambda)) \cap \mathscr{M},
$$

e, correspondentemente em $\mathscr{I}$,

$$
\mathscr{D}_{p, q}^{+}(\lambda) \doteq\left(\lambda_{p, q}^{\bar{g}}\right)^{-1}((\lambda-\epsilon,+\infty)) \cap \mathscr{I}, \mathscr{D}_{p, q}^{-}(\lambda) \doteq\left(\lambda_{p, q}^{\bar{g}}\right)^{-1}((-\infty, \epsilon-\lambda)) \cap \mathscr{I} .
$$

Seja $\mathfrak{A}$ uma teoria quântica localmente covariante estendida regular, satisfazendo as seguintes hipóteses:

(a) A realização de $\mathfrak{A}$ em $(\mathscr{M}, g)$ é localmente causal com respeito à coleção das cunhas $\mathscr{W}(\mathscr{M}, g)$; 
(b) $\mathfrak{A}$ é fracamente primitivamente causal com respeito às cunhas de $(\mathscr{M}, g)$ (garantindo destarte a possibilidade de impor uma evolução temporal, sujeita a condições de contorno);

(c) Existe um espaço de estados $\mathfrak{S}$ tal que $\mathfrak{S}(\mathscr{M}, g)$ possui um representante $\omega$ primário satisfazendo a propriedade de definiteza local holográfica (definida no item (iii) da Proposição 4.4, página 80) e tal que seu fólio local com respeito às subregiões causalmente convexas de cunhas está contido em $\mathfrak{S}(\mathscr{M}, g)$.

Omitimos no que se segue a notação de restrição da métrica $g$ e de quociente pelos *_ ideais não-triviais que anulam os propagadores ( ${ }^{*}$-ideais de contorno), por simplicidade, tendo em mente sempre os Teoremas de ${ }^{*}$-Isomorfismo. Segue de (b) que $\mathfrak{A}\left(\mathscr{W}_{p, q}^{ \pm}(\lambda), g\right)$ é ${ }^{*}$-isomorfa a $\mathfrak{A}\left(\mathscr{W}_{p, q}\right)$ para todo $\gamma,(p, q) \in \mathscr{D}(\mathscr{I})$, modulo ${ }^{*}$-ideais de contorno. Mais ainda, podemos definir o germe $\mathfrak{A}\left(\partial_{ \pm} \mathscr{W}_{p, q}\right)$ de $\mathfrak{A}\left(\mathscr{W}_{p, q}\right)$ nos horizontes passado e futuro $\partial_{ \pm} \mathscr{W}_{p, q}$ pelos limites projetivos (modulo ${ }^{*}$-ideais de contorno)

$$
\mathfrak{A}\left(\partial_{ \pm} \mathscr{W}_{p, q}\right) \doteq \lim _{\lambda \nearrow+\infty, \mathfrak{A}} \mathfrak{A}\left(\mathscr{W}_{p, q}^{ \pm}(\lambda), g\right)
$$

lembrando que a relação de equivalência que define o limite é dada pela identificação (modulo *-ideais de contorno) $A \in \mathfrak{A}\left(\mathscr{W}_{p, q}^{ \pm}(\lambda), g\right) \sim\left(\mathfrak{A}_{\mathscr{W}_{p, q}^{ \pm}(\lambda), \mathscr{W}_{p, q}^{ \pm}\left(\lambda^{\prime}\right)}\right)^{-1}(A)$, para todo $\lambda^{\prime} \geq \lambda$, similarmente ao caso dos germes de álgebras locais em superfícies de CAUCHY, definidos no Capítulo 3 (ver a fórmula (3.6), página 63).

Com as definições acima, se $r \in\left(\lambda_{p, q}^{\bar{g}}\right)^{-1}\left(\lambda_{0}\right) \cap \mathscr{I}$ e $r \ll \mathscr{I} s \leq \mathscr{I} q$, obviamente $\mathscr{W}_{r, s} \subset \mathscr{W}_{p, q}^{+}\left(\lambda_{0}\right)$ e, portanto, $\mathscr{W}_{u_{p, q}^{\lambda}(r), u_{p, q}^{\lambda}(s)} \subset \mathscr{W}_{p, q}^{+}\left(\lambda_{0}+\lambda\right)$ para todo $\bar{\lambda} \geq 0$.

DefiniçÃo 4.9 Sejam $p \ll q \in \mathscr{M}$ in $(r)$ para algum $r \in \mathscr{I}$, onde assume-se que $\mathscr{M}$ in $(r)$ é dotado das operações de espaço vetorial de $\mathbb{R}^{1, d-2}$. A álgebra de escala futura $\mathfrak{A}^{+}\left(\mathscr{W}_{p, q}\right)$ (resp. passada $\mathfrak{A}^{-}\left(\mathscr{W}_{p, q}\right)$ ) em $\mathscr{W}_{p, q}$ consiste das funções uniformemente limitadas em norma $(0,1] \ni \theta \mapsto \underline{A}^{+}(\theta) \doteq A_{\theta}^{+} \in \mathfrak{A}\left(\mathscr{W}_{p, q}^{+}\left(f_{p,+}^{-1}(\theta)\right), g\right)$ (resp. $(0,1] \ni \theta \mapsto$ $\left.\underline{A}^{-}(\theta) \doteq A_{\theta}^{-} \in \mathfrak{A}\left(\mathscr{W}_{p, q}^{-}\left(f_{q,-}^{-1}(\theta)\right), g\right)\right)$ satisfazendo $A_{1}^{+} \doteq A^{+} \in \mathfrak{A}\left(\mathscr{W}_{r, s}\right), r \in \mathscr{W}_{p, q}^{+}\left(\lambda_{0}\right)$ para algum $\lambda_{0}$ implica $A_{\theta}^{+} \in \mathfrak{A}\left(\mathscr{W}_{p, q}^{+}\left(\lambda_{0}+f_{r,+}^{-1}(\theta)\right), g\right)$ (resp. $A_{1}^{-} \doteq A^{-} \in \mathfrak{A}\left(\mathscr{W}_{r, s}\right), s \in \mathscr{W}_{p, q}^{-}\left(\lambda_{0}\right)$ para algum $\lambda_{0}$ implica $\left.A_{\theta}^{-} \in \mathfrak{A}\left(\mathscr{W}_{p, q}^{-}\left(\lambda_{0}+f_{s,-}^{-1}(\theta)\right), g\right)\right)$, para todo $p \leq \mathscr{I} r \ll \mathscr{I} s \leq \mathscr{I} q$, $\theta \in(0,1]$, onde $f_{r,+}$ e $f_{s,-}$ são definidas na página 79. As operações algébricas são definidas ponto a ponto, e a $C^{*}$-norma é dada por $\left\|\underline{A}^{ \pm}\right\| \doteq \sup _{0<\theta \leq 1}\left\|A_{\theta}^{ \pm}\right\|$.

A definição mais geral dada acima lida com a perda da localização "espacial" causada pela ação dos propagadores

$$
\alpha_{\lambda, \lambda^{\prime}}^{ \pm} \doteq\left(\mathfrak{A} i_{\mathscr{W}_{p, q}^{ \pm}\left(\lambda^{\prime}\right), \mathscr{W}_{p, q}}\right)^{-1} \circ \mathfrak{A} i_{\mathscr{W}_{p, q}^{ \pm}(\lambda), \mathscr{W}_{p, q}},
$$

que podem ser então usados para construir elementos de $\mathfrak{A}^{ \pm}\left(\mathscr{W}_{p, q}\right)$ (modulo *-ideais de contorno) da mesma forma que empregamos $\mathfrak{A} u_{p, q}^{\lambda}$ no caso de $A d S_{d}$. Supomos, de 
acordo, que os propagadores (4.14) são fortemente contínuos na norma das álgebras de escala.

Notamos agora um ponto muito importante: as imagens dos elementos das álgebras de escala pertencem às álgebras intrínsecas, e não a suas imagens em $\mathfrak{A}\left(\mathscr{W}_{p, q}\right)$, que identificam elementos que diferem por um elemento de um *-ideal de contorno. Isso elimina uma aparente contradição entre os germes de álgebras locais nos horizontes passado e futuro e o princípio de definiteza local holográfica. As aplicações

$$
A_{\theta}^{+} \mapsto I_{p, q}^{+}\left(\underline{A}^{+}\right)(\theta) \doteq \mathfrak{A} i_{\mathscr{W}_{p, q}^{+}\left(f_{p,+}^{-1}(\theta)\right), \mathscr{W}_{p, q}}\left(A_{\theta}^{+}\right)
$$

e

$$
A_{\theta}^{-} \mapsto I_{p, q}^{-}\left(\underline{A}^{-}\right)(\theta) \doteq \mathfrak{A} i_{\mathscr{W}_{p, q}^{-}\left(f_{q,-}^{-1}(\theta)\right), \mathscr{W}_{p, q}}\left(A_{\theta}^{-}\right)
$$

definem *-morfismos das álgebras de escala nas funções uniformemente limitadas de $(0,1] \ni \theta$ em $\mathfrak{A}\left(\mathscr{W}_{p, q}\right)$ e preservam a localização "temporal". Definindo o levantamento $\omega_{\theta}^{ \pm}$do nosso estado de referência $\omega$ a $I_{p, q}^{ \pm}\left(\mathfrak{A}^{ \pm}\left(\mathscr{W}_{p, q}\right)\right)$ na escala $\theta$ da mesma maneira que no caso AdS, podemos finalmente impor nossas condições de contorno:

(d)

$$
\operatorname{Ann} \mathfrak{S}\left(\mathscr{W}_{p, q}^{ \pm}(\lambda), g\right) \doteq \bigcap_{\omega^{\prime} \in \mathfrak{S}\left(\mathscr{W}_{p, q}^{ \pm}(\lambda), g\right)} \operatorname{Ann} \omega^{\prime} \supset \operatorname{Ker} \mathfrak{A} i_{\mathscr{W}_{p, q}^{ \pm}(\lambda), \mathscr{W}_{p, q}}
$$

para todo $\lambda \in \mathbb{R}$, e os limites de escala $\omega_{0, \iota}^{ \pm}$definem representações GNS ligadas por *-isomorfismos de rede com respeito à coleção das cunhas em $(\mathscr{M}, g)$, e ${ }^{*}$-isomorfas à *-representação de $\mathfrak{A}\left(\partial_{ \pm} \mathscr{W}_{p, q}\right)$ induzida pelo quociente por $\operatorname{Ann} \mathfrak{S}\left(\partial_{ \pm} \mathscr{W}_{p, q}^{ \pm}\right)$(esse espaço de estados é definido empregando-se a contravariância de $\mathfrak{S}$ e a universalidade de limites projetivos). A ação *-automorfa $(0,1] \ni \mu \mapsto \delta_{p, q}^{\mu, \pm}$ das transformações de escala em $\pi_{0, \iota}\left(I_{p, q}^{ \pm}\left(\mathfrak{A}^{ \pm}\left(\mathscr{W}_{p, q}\right)\right)\right)$ é unitariamente implementada no limite de escala na forma

$$
\pi_{0, \iota}\left(\delta_{p, q}^{\exp \left( \pm \kappa_{ \pm} \lambda\right), \pm}\left(I_{p, q}^{ \pm}\left(\underline{A}^{ \pm}\right)\right)\right)=\operatorname{Ad} \Delta_{p, q}^{\frac{i}{2 \pi} \lambda} \pi_{0, \iota}\left(I_{p, q}^{ \pm}\left(\underline{A}^{ \pm}\right)\right)
$$

onde $\Delta_{p, q}$ é o operador modular de TOMITA-TAKESAKI associado a $\omega_{0, \iota}$ (que é, portanto, essencialmente único para todo $\iota$ ), e $\kappa_{ \pm}=\mp 1$ é a gravidade de superfície assintótica passada/futura de $\mathscr{W}_{p, q}$, obtida no Teorema 2.6 (página 53).

A condição (d), embora bastante complicada, é naturalmente motivada pela Proposição 4.4 e pelo Teorema 2.6 no caso de $A d S_{d}$. Juntamente com (c), ela não só é comum a todos os estados no fólio local de $\omega$ como também implica que as álgebras de von Neumann $\pi_{\omega}\left(\mathfrak{A}\left(\mathscr{W}_{p, q}\right)\right)^{\prime \prime}$ são tipo III, analogamente ao item (iii) da Proposição 4.4. A continuidade assumida dos propagadores, juntamente com a covariância local, 
garante que os limites de escala de diferentes cunhas vivem no mesmo espaço de HıLBERT, como no item (ii). O caráter geométrico em $\mathscr{I}$ da ação dos grupos modulares, por sua vez, segue do Teorema 4.1. Em virtude do valor assintótico que encontramos para as gravidades de superfície das cunhas e das relações de comutação entre os diferentes grupos modulares, resultantes do Teorema 4.1, temos que os grupos modulares agem geometricamente exatamente como transformações conformes em $\mathscr{I}$, e o limite de escala $\omega_{0, \iota}$ define um vácuo [BS93] conformalmente invariante no pré-cofeixe $\mathscr{D}_{p, q} \mapsto \pi_{0, \iota}\left(\left(I_{p, q}^{ \pm}\left(\mathfrak{A}^{ \pm} \circ \rho_{(\mathscr{M}, g)}^{-1}\right)\left(\mathscr{D}_{p, q}\right)\right)^{\prime \prime}\right.$. Em suma, o limite de escala que definimos perde toda a informação geométrica acerca de $(\mathscr{M}, g)$, e poderia ser tomado como uma teoria quântica Rehren-dual a uma teoria quântica em $A d S_{d}$. Mais ainda, a implementação das isometrias assintóticas só ocorre no limite de escala. Notamos aqui o forte parentesco da construção acima com a holografia na frente de luz proposta por SCHROER [Sch06, Sch05].

Enfatizamos uma definição dinâmica como a Definição 4.9 para as álgebras de escala em cunhas AAdS, ao invés da Definição mais simples 4.4 dada no caso de $A d S_{d}$ (que, em princípio é também aplicável aqui) por várias razões. Primeiro, a condição de contorno (d) dada nos moldes da Definição 4.9 enfatiza o caráter de retorno ao equilíbrio dual ao limite de escala, e se estende a contextos mais gerais (i.e., diamantes relativamente compactos em espaços-tempos causalmente simples), tal como a construção de funções tempo globais no Capítulo 2. Segundo, abre-se a possibilidade de sondarmos dinamicamente desvios do comportamento de equilíbrio térmico da mesma forma que sondamos desvios do comportamento crítico (i.e., invariante de escala) por meio de, digamos, expansões de produto de operadores [FH81, Wei96, Bos05, BOR02].

\subsection{Análise holográfica dos setores de superseleção}

O desenvolvimento que conclui esta Tese consiste em mostrar como as propriedades geométricas de espaços-tempos AAdS não-triviais (i.e., diferentes de $A d S_{d}$ ) dentro das hipóteses empregadas ao longo do Capítulo 1 modificam de maneira radical não só a implementação de simetrias geométricas, mas também das simetrias internas, codificadas na estrutura de setores de superseleção. No que se segue, faremos o seguinte conjunto de hipóteses sobre os espaços-tempos e as teorias quânticas localmente covariantes de nosso interesse:

(i) $(\mathscr{M}, g)$ é um espaço-tempo AAdS satisfazendo as hipóteses da Proposição 1.8;

(ii) $\mathfrak{A}$ é uma teoria quântica localmente covariante estendida, cuja realização em $(\mathscr{M}, g)$ satisfaz causalidade local com respeito a $\mathscr{W}(\mathscr{M})$; 
(iii) Temos à nossa disposição um espaço de estados $\mathfrak{S}_{0}$, dito de referência, tal que quaisquer $\omega, \omega^{\prime} \in \mathfrak{S}_{0}\left(\mathscr{W}_{p, q}\right)$ satisfazem a condição (3.8) (página 66) para todo $(p, q) \in \mathscr{D}(\mathscr{I})$. Em particular, como vimos, as álgebras de VON NEUMANN $\pi_{\omega}\left(\mathfrak{A}\left(\mathscr{W}_{p, q}\right)\right)^{\prime \prime}, \omega \in \mathfrak{S}_{0}\left(\mathscr{W}_{p, q}\right)$, são todas ${ }^{*}$-isomorfas entre si.

(iv) $\mathfrak{S}_{0}\left(\mathscr{W}_{p, q}\right)$ possui um representante $\omega_{0}$, (também) dito de referência, que satisfaz:

- A propriedade de ReEH-Schlieder, i.e., o vetor cíclico $\Omega_{0}$ no espaço de HilberT GNS $\mathscr{H}_{0} \doteq \mathscr{H}_{\omega_{0}}$ associado a $\omega_{0}$ é separante para $\pi_{0}\left(\mathfrak{A}\left(\mathscr{W}_{p, q}\right)\right)^{\prime \prime}$, onde $\pi_{0} \doteq \pi_{\omega_{0}}$ é a ${ }^{*}$-representação GNS associada a $\omega_{0}$;

- A propriedade de definiteza local holográfica;

- A propriedade (III), i.e., $\pi_{0}\left(\mathfrak{A}\left(\mathscr{W}_{p, q}\right)\right)^{\prime \prime}$ é tipo III para todo $(p, q) \in \mathscr{D}(\mathscr{I})$ (ver Apêndice B, Definição B.5, página 135).

Condições adicionais serão eventualmente acrescentadas ao longo dos desenvolvimentos a seguir. Note que os estados $\omega_{0}$ satisfazendo as condições de contorno $(\mathrm{c})-(\mathrm{d})$ impostas na Seção anterior satisfazem as propriedades enumeradas pela hipótese (iv).

OBSERVAÇÃO 4.10 As hipóteses (iii) e (iv) sobre o espaço de estados de referência $\mathfrak{S}_{0}$ são similares às adotadas por BRUNETTI e RUzzi [BRO'7] em sua formulação localmente covariante para a Teoria Algébrica de Setores de Superseleção. Não adotaremos, contudo, a abordagem desse trabalho por simplicidade, uma vez que abordaremos apenas elementos básicos desta Teoria no que se segue.

\subsubsection{Uma digressão. Dualidade de HAAG e elementos da Teoria Algébrica de Setores de Superseleção}

O fato de que a coleção $\mathscr{W}\left(A d S_{d}\right)$ das cunhas em $A d S_{d}$ é fechada pela operação de complemento causal (mais precisamente, as fórmulas (1.29) são válidas) garante que não apenas causalidade local é preservada pela bijeção de REHREN, como também a possível maximalidade das álgebras locais com respeito a esta propriedade. Veremos na próxima Subseção que, devido à geometria das cunhas em espaços-tempos AAdS não-triviais, essa maximalidade ganha contornos de um teorema "no-go" acerca da não-trivialidade das álgebras locais no interior de tais espaços-tempos, com implicações sobre a estrutura dos setores de superseleção. Entretanto, antes disso, formularemos de maneira precisa essa hipótese se maximalidade, e ilustraremos seu papel na determinação desta estrutura.

DEFINIÇÃO 4.11 Seja $\mathfrak{A}$ uma teoria quântica localmente covariante estendida, $(\mathscr{M}, g) \in$ Obj $\mathscr{S} t c_{d}, \pi$ uma ${ }^{*}$-representação de $\mathfrak{A}_{\mathscr{M}}$ no espaço de Hilbert $\mathscr{H}$, e $\mathscr{Q}(\mathscr{M}, g)$ uma coleção de abertos causalmente completos de $(\mathscr{M}, g)$. Dizemos que a realização de 
$\mathfrak{A}$ em $(\mathscr{M}, g)$ é $\pi$-HAAG-dual com respeito a $\mathscr{Q}(\mathscr{M}, g)$ se as álgebras de VON NEUMANN associadas a $\pi$ e localizadas em elementos de $\mathscr{Q}(\mathscr{M}, g)$ são maximalmente localmente causais, i.e., $\pi(\mathfrak{A}(\mathscr{O}))^{\prime \prime}=\pi\left(\mathfrak{A}\left(\mathscr{O}^{\prime}\right)\right)^{\prime}$, onde $\pi(\mathfrak{A}(\mathscr{O}))^{\prime}$ denota o comutante de $\pi(\mathfrak{A}(\mathscr{O}))$ em $\mathscr{H}$ (ver no Apêndice $B$ a discussão que precede imediatamente a Definição B.4, página 132) - lembrar que causalidade local de $\mathfrak{A}$ implica apenas que $\pi\left(\mathfrak{A}\left(\mathscr{O}^{\prime}\right)\right)^{\prime \prime} \subset \pi(\mathfrak{A}(\mathscr{O}))^{\prime}$. Mais em geral, dizemos que a realização de $\mathfrak{A}$ em $(\mathscr{M}, g)$ $e ́$ essencialmente $\pi$-HAAG-dual com respeito a $\mathscr{Q}(\mathscr{M}, g)$ se a extensão HAAG-dual $\mathscr{Q}(\mathscr{M}, g) \ni \mathscr{O} \mapsto \pi(\mathfrak{A}(\mathscr{O}))^{d} \doteq \pi\left(\mathfrak{A}\left(\mathscr{O}^{\prime}\right)\right)^{\prime}$ da realização de $\mathfrak{A}$ em $(\mathscr{M}, g)$ na representação $\pi$ é localmente causal, i.e., $\pi\left(\mathfrak{A}\left(\mathscr{O}_{1}\right)\right)^{d} \subset\left(\pi\left(\mathfrak{A}\left(\mathscr{O}_{2}\right)\right)^{d}\right)^{\prime}$ para todo par $\mathscr{O}_{1}, \mathscr{O}_{2} \in \mathscr{Q}(\mathscr{M}, g)$ tal que $\mathscr{O}_{1} \perp \mathscr{O}_{2}$. Uma ${ }^{*}$-representação $\pi$ de $\mathfrak{A}_{\mathscr{M}}$ é dita ser (resp. essencialmente) HAAG-dual se a realização de $\mathfrak{A}$ em $(\mathscr{M}, g)$ for (resp. essencialmente) $\pi$-HAAG-dual.

OBSERVAÇÃO 4.12 Há uma sutileza na definição de dualidade de HAAG essencial: é imediato ver que, se $\mathscr{Q}(\mathscr{M}, g)$ for fechado por complementos causais, i.e., $\mathscr{O} \in$ $\mathscr{Q}(\mathscr{M}, g) \Leftrightarrow \mathscr{O}^{\prime} \in \mathscr{Q}(\mathscr{M}, g)$, então dualidade de HAAG e dualidade de HAAG essencial com respeito a $\mathscr{Q}(\mathscr{M}, g)$ coincidem. Assim, os efeitos interessantes que surgem quando a realização de $\mathfrak{A}$ em $(\mathscr{M}, g)$ é apenas essencialmente $\pi$-HAAG-dual com respeito a $\mathscr{Q}(\mathscr{M}, g)$ só aparecem quando este último não é fechado por complementos causais. $O$ exemplo típico é $(\mathscr{M}, g)=\mathbb{R}^{1, d-1}$ e $\mathscr{Q}\left(\mathbb{R}^{1, d-1}\right)=\left\{I^{+}(p) \cap I^{+}(q): p \ll q \in \mathbb{R}^{1, d-1}\right\}$.

A quebra da dualidade de HAAG implica, por exemplo, na quebra espontânea de simetrias internas para a representação induzida por um vácuo em teorias de observáveis locais no espaço-tempo de Minkowski [Haa96]. A razão, em termos intuitivos, é a seguinte: a presença de simetrias internas, manifestas na estrutura dos multipletos de partículas que aparecem em processos de espalhamento, é codificada nas álgebras locais por meio dos "intertwiners" 10 entre as representações unitárias do grupo de simetrias internas. A dualidade de HAAG garante que há "intertwiners" suficientes para reconstruir completamente a teoria de representação deste grupo (= estrutura dos setores de superseleção) e, portanto, o próprio grupo - este resultado, considerado um dos grandes triunfos da abordagem algébrica para a Teoria Quântica de Campos, foi demonstrado por Doplicher e RoBERTs [Ara99, Haa96], e constitui uma formulação abstrata para a dualidade entre grupos compactos e suas representações irredutíveis (teorema de TANNAKA-KREIN).

Se apenas dualidade de HAAG essencial é válida, é possível reconstruir a parte das simetrias internas que não sofre quebra espontânea repetindo o procedimento de DoPLICHER e RoBERTs para a extensão HAAG-dual das álgebras locais, conforme a Definição

\footnotetext{
${ }^{10}$ Dado um grupo $G$ e duas representações unitárias $G \ni g \mapsto U_{1}(g), U_{2}(g)$ de $G$ respectivamente nos espaços de Hilbert $\mathscr{H}_{1}$ e $\mathscr{H}_{2}$, um "intertwiner" entre $U_{1}$ e $U_{2}$ é um operador linear limitado $T: \mathscr{H}_{1} \rightarrow \mathscr{H}_{2}$ tal que $T U_{1}(g)=U_{2}(g) T$ para todo $g \in G$. Em particular, tal definição se aplica, como será freqüentemente utilizado, a *-representações de *álgebras.
} 
4.11. O procedimento para identificar a parte espontaneamente quebrada das simetrias internas é, todavia, um pouco mais sutil [Haa96].

Vamos agora precisar melhor como a estrutura de setores de superseleção é codificada na realização de $\mathfrak{A}$ em $(\mathscr{M}, g)$, seguindo o tratamento em [Ara99] e [GLRV01], a que nos referimos para detalhes. Sejam $\mathfrak{A},(\mathscr{M}, g)$ e $\mathscr{Q}(\mathscr{M}, g)$ como na Definição 4.11, e $\pi_{0}$ uma *-representação HAAG-dual de $\mathfrak{A}_{\mathscr{M}}$ no espaço de HilberT $\mathscr{H}_{0}$, que adotaremos como referência. Dizemos que uma ${ }^{*}$-representação $\pi$ de $\mathfrak{A}_{\mathscr{M}}$ num espaço de HiLberT $\mathscr{H}$ satisfaz o critério $D H R{ }^{11}$ com respeito a $\mathscr{Q}(\mathscr{M}, g)$ se, para todo $\mathscr{O} \in \mathscr{Q}(\mathscr{M}, g)$, $\pi \uparrow_{\mathfrak{A}\left(\mathscr{O}^{\prime}\right)}$ é unitariamente equivalente a $\pi_{0} \uparrow_{\mathfrak{A}\left(\mathscr{O}^{\prime}\right)}$. Seja, então, $V$ um operador unitário de $\mathscr{H}$ em $\mathscr{H}_{0}$ tal que $V \pi(A) V^{*}=\pi_{0}(A)$ para todo $A \in \mathfrak{A}\left(\mathscr{O}^{\prime}\right)$. Defina o seguinte *-morfismo de $\pi_{0}\left(\mathfrak{A}_{\mathscr{M}}\right)$ em $\mathscr{B}\left(\mathscr{H}_{0}\right)$ :

$$
\rho\left(\pi_{0}(A)\right) \doteq V \pi(A) V^{*}
$$

Por construção, $\rho \circ \pi_{0}$ é unitariamente equivalente a $\pi$. Assumindo, por ora, que $\mathscr{Q}(\mathscr{M}, g)$ é direcionado mediante o ordenamento parcial induzido por inclusões de conjuntos, considere $\mathscr{O}_{1}, \mathscr{O}_{2} \in \mathscr{Q}(\mathscr{M}, g)$ tais que $\mathscr{O}_{2} \supset \mathscr{O}, \mathscr{O}_{1}$. Então,

$$
\left[\rho\left(\pi_{0}(A)\right), \rho\left(\pi_{0}(B)\right)\right]=\left[\rho\left(\pi_{0}(A)\right), \pi_{0}(B)\right]=0
$$

para todo $A \in \mathfrak{A}\left(\mathscr{O}_{1}\right)$ e $B \in \mathfrak{A}\left(\mathscr{O}_{2}^{\prime}\right) \subset \mathfrak{A}\left(\mathscr{O}^{\prime}\right)$. Devido à dualidade de HAAG, temos que $\rho\left(\pi_{0}(A)\right) \in \pi_{0}\left(\mathfrak{A}\left(\mathscr{O}_{2}^{\prime}\right)\right)^{\prime}=\pi_{0}\left(\mathfrak{A}\left(\mathscr{O}_{2}\right)\right)^{\prime \prime}$. Segue que, pela definição de álgebra quase-local, $\rho \circ \pi_{0}\left(\mathfrak{A}_{\mathscr{M}}\right) \subset \rho\left(\pi_{0}\left(\mathfrak{A}_{\mathscr{M}}\right)^{\prime \prime}\right) \subset \pi_{0}\left(\mathfrak{A}_{\mathscr{M}}\right)^{\prime \prime}$.

O argumento acima é uma linha de raciocínio recorrente na Teoria Algébrica de Setores de Superseleção. Com ele, podemos definir o transporte de *-endomorfismos localizados de $\pi_{0}\left(\mathfrak{A}_{\mathscr{M}}\right)^{\prime \prime}$ sem o auxílio da implementação de um grupo de translações. Mais precisamente, temos os seguintes fatos:

- Dados dois *-endomorfismos $\rho_{1}, \rho_{2}$ localizados respectivamente em $\mathscr{O}_{1}$ e $\mathscr{O}_{2}$ e tais que as representações $\rho_{1} \circ \pi_{0}$ e $\rho_{2} \circ \pi_{0}$ são unitariamente equivalentes, i.e., $\rho_{1} \circ \pi_{0}(A)=U \rho_{2} \circ \pi_{0}(A) U^{*}$, então $U \in \pi_{0}\left(\mathfrak{A}\left(\mathscr{O}_{3}^{\prime}\right)\right)^{\prime}$, onde $\mathscr{O}_{3} \supset \mathscr{O}_{1}, \mathscr{O}_{2}$. Ou seja, *-endomorfismos localizados em $\mathscr{O}$ podem ser transportados para qualquer outro elemento de $\mathscr{Q}(\mathscr{M}, g)$ por meio de "intertwiners" locais.

- A composição $\rho_{1} \circ \rho_{2}$ é claramente localizada em $\mathscr{O}_{3} \supset \mathscr{O}_{1}, \mathscr{O}_{2}$, onde $\rho_{i}$ é localizado em $\mathscr{O}_{i}$. Mais ainda, se $\mathscr{O}_{1} \perp \mathscr{O}_{2}$, então $\rho_{1} \circ \rho_{2}=\rho_{2} \circ \rho_{1}$.

\footnotetext{
${ }^{11}$ DHR responde pelos nomes de Doplicher, HaAg e Roberts.
} 
Um *-endomorfismo $\rho$ é dito irredutível se a *-representação $\rho \circ \pi_{0}$ o for. Neste caso, dizemos que o conjunto dos estados puros no fólio de $\rho \circ \pi_{0}$ é o setor (de superseleção) associado a $\rho$.

Denotamos o espaço de BANACH dos "intertwiners" (locais) entre os *-endomorfismos localizados $\rho$ e $\sigma$ por $(\rho, \sigma) \doteq\{U: U \rho(A)=\sigma(A) U, \forall A\}$. Dados $T \in(\rho, \sigma), T^{\prime} \in\left(\rho^{\prime}, \sigma^{\prime}\right)$ podemos definir o produto tensorial $T \otimes T^{\prime} \doteq T \rho\left(T^{\prime}\right) \in\left(\rho \circ \rho^{\prime}, \sigma \circ \sigma^{\prime}\right)$. Podemos provar que, se $T$ e $T^{\prime}$ são causalmente disjuntos, então $T \otimes T^{\prime}=T^{\prime} \otimes T$. Segue daí que, se $\sigma$ e $\sigma^{\prime}$ são localizados em regiões causalmente disjuntas, então $\left(T^{\prime} \otimes T\right)^{*} \circ\left(T \otimes T^{\prime}\right) \doteq \epsilon\left(\rho, \rho^{\prime}\right) \epsilon$ $\left(\rho \circ \rho^{\prime}, \rho^{\prime} \circ \rho\right)$ é independente de $\sigma, \sigma^{\prime}$ ou de $T, T^{\prime}$. Generalizando para $n$ *-endomorfismos localizados $\rho_{i}, i=1, \ldots, n$, obtemos "intertwiners" $\epsilon_{p}\left(\rho_{1}, \ldots, \rho_{n}\right)$ para cada permutação $p$ de $\{1, \ldots, n\}$, de forma tal que $\epsilon_{p^{\prime}}\left(\rho_{1}, \ldots, \rho_{n}\right) \circ \epsilon_{p}\left(\rho_{1}, \ldots, \rho_{n}\right)=\epsilon_{p^{\prime} \circ p}\left(\rho_{1}, \ldots, \rho_{n}\right)$. Desta forma, se $\rho_{1}=\cdots=\rho_{n}=\rho$ a aplicação $S_{n} \ni p \mapsto \epsilon_{p}(\rho, \ldots, \rho) \in \rho^{n}\left(\pi_{0}\left(\mathfrak{A}_{\mathscr{M}}\right)\right)^{\prime}$ constitui uma representação unitária do grupo $S_{n}$ de permutações de $n$ elementos em $\rho^{n} \circ \pi_{0}$. Os operadores $\epsilon$ são denominados operadores de estatística, pois refletem a relação spin-estatística dos setores de superseleção. São precisamente tais operadores, juntamente com estruturas adicionais (conjugados, inversas à esquerda), permitem reconstruir o grupo de simetrias internas e os multipletos de partículas.

Da mesma forma que a composição de *-endomorfismos corresponde ao produto tensorial de representações, podemos descrever subrepresentações e somas diretas por subobjetos e somas diretas de *-endomorfismos: dizemos que $\sigma$ é subobjeto de $\rho$ se existe uma isometria $W \in(\sigma, \rho)$ (i.e., $W^{*} W=\mathbb{1}$ e, portanto, $W W^{*}$ é uma projeção), e dizemos que $\tau$ é soma direta de $\rho$ e $\sigma$ se existem isometrias $V \in(\rho, \tau), W \in(\sigma, \tau)$ tais que $V V^{*}+W W^{*}=\mathbb{1} \mathrm{e}$, portanto, $\tau()=.V \rho(.) V^{*}+W \sigma(.) W^{*}$. Para garantir que estes *endomorfismos permanecem localizados, precisamos invocar a chamada propriedade $B$ de Borchers, válida, por exemplo, para fatores tipo III: dados $\mathscr{O}_{1}, \mathscr{O}_{2} \in \mathscr{Q}(\mathscr{M}, g)$ tais que $\overline{\mathscr{O}_{1}} \subset \mathscr{O}_{2}$ e uma projeção $E \in \pi_{0}\left(\mathfrak{A}\left(\mathscr{O}_{1}\right)\right)^{\prime \prime}$, existe uma isometria $W \in \pi_{0}\left(\mathfrak{A}\left(\mathscr{O}_{1}\right)\right)^{\prime \prime}$ tal que $W W^{*}=E$. No caso de fatores tipo III, podemos tomar $\mathscr{O}_{2}=\mathscr{O}_{1}$.

Quando $\mathscr{Q}(\mathscr{M}, g)$ não é direcionado, o *-morfismo $\rho$ definido em (4.18) é definido apenas no pré-cofeixe $\mathscr{Q}(\mathscr{M}, g) \ni \mathscr{O} \subset \mathscr{O}_{1} \mapsto \pi_{0}\left(\mathfrak{A}\left(\mathscr{O}_{1}\right)\right)^{\prime \prime}$, e satisfaz aqui $A \in \mathfrak{A}\left(\mathscr{O}_{1}\right) \Rightarrow$ $\rho\left(\pi_{0}(A)\right) \in \pi_{0}\left(\mathfrak{A}\left(\mathscr{O}_{1}\right)\right)^{\prime \prime}$.

\subsubsection{Dualidade de HAAG (essencial) em espaços-tempos (A)AdS}

Agora, com a linguagem adequada em mãos, voltemos a discutir a questão suscitada no primeiro parágrafo da Subseção anterior. Consideremos um espaço-tempo AAdS $(\mathscr{M}, g)$, uma teoria quântica localmente covariante estendida $\mathfrak{A}$ realizada em 
$(\mathscr{M}, g)$ e $\pi_{0}$ uma ${ }^{*}$-representação de referência de $\mathfrak{A}_{\mathscr{M}}$, que assumimos irredutível por simplicidade. Novamente, tanto $\mathscr{D}\left(A d S_{d}\right)$ quanto $\mathscr{W}\left(A d S_{d}\right)$ são fechados por complementos causais, em razão das fórmulas (1.29) (página 14). Empregando a bijeção de REHREN (1.32) (página 16), segue automaticamente que a realização de $\mathfrak{A}$ em $A d S_{d}$ é $\pi_{0}$-HAAG-dual com respeito a $\mathscr{W}\left(A d S_{d}\right)$ se e somente se a realização REHREN-dual de $\mathfrak{A}$ em $E S U_{d-1}$ o for em relação a $\mathscr{D}\left(A d S_{d}\right)$ - lembrar também que, em ambos os casos, dualidade de HAAG e dualidade de HAAG essencial coincidem.

Vejamos agora o que ocorre se $(\mathscr{M}, g)$ é um espaço-tempo AAdS não-trivial, satisfazendo as hipóteses do Teorema 1.3 , página 17 . Neste caso, $\mathscr{W}(\mathscr{M}, g)$ não é mais fechado por complementos causais, havendo assim uma diferença fundamental entre dualidade de HAAG e dualidade de HAAG essencial. Não obstante, ainda vale o seguinte

LEMA $4.5 S e \pi_{0}$ é (essencialmente) HAAG-dual com respeito a $\mathscr{D}(\mathscr{M}, g)$, então é HAAG-dual com respeito a $\mathscr{W}(\mathscr{M}, g)$ e $\pi_{0}\left(\mathfrak{A}\left(\mathscr{W}_{p, \bar{q}}\right)\right)^{\prime \prime}=\pi_{0}\left(\mathfrak{A}\left(\mathscr{W}_{q, \bar{p}}^{\prime}\right)\right)^{\prime \prime}=\pi_{0}\left(\mathfrak{A}\left(\mathscr{W}_{\bar{p}, q}\right)^{\prime}\right)^{\prime}$ para todo par $p, q \in \mathscr{I}$ tal que $(p, \bar{q}) \in \mathscr{D}(\mathscr{I})$, onde $\bar{p}$ é o antípoda de $p$ (ver fórmulas (1.27)-(1.28) e a discussão que as precedem na página 12).

Prova. $\quad$ Dualidade de HAAG essencial com respeito a $\mathscr{D}(\mathscr{M}, g)$ não só é equivalente a dualidade de HAAG (pois a coleção dos diamantes em $\mathscr{I}$ é fechada por complementos causais), como implica em dualidade de HAAG para cunhas, pois $\pi_{0}\left(\mathfrak{A}\left(\mathscr{D}_{p, \bar{q}}\right)\right)^{\prime \prime}=\pi_{0}\left(\mathfrak{A}\left(\mathscr{W}_{p, \bar{q}}\right)\right)^{\prime \prime} \subset \pi_{0}\left(\mathfrak{A}\left(\mathscr{W}_{p, \bar{q}}^{\prime}\right)\right)^{\prime} \subset \pi_{0}\left(\mathfrak{A}\left(\mathscr{W}_{q, \bar{p}}\right)\right)^{\prime}=\pi_{0}\left(\mathfrak{A}\left(\mathscr{D}_{q, \bar{p}}\right)\right)^{\prime}=$ $\pi_{0}\left(\mathfrak{A}\left(\mathscr{D}_{p, \bar{q}}^{\prime}\right)\right)^{\prime}$. A última assertiva segue das igualdades $\pi_{0}\left(\mathfrak{A}\left(\mathscr{W}_{p, \bar{q}}\right)\right)^{\prime \prime}=\pi_{0}\left(\mathfrak{A}\left(\mathscr{D}_{p, \bar{q}}\right)\right)^{\prime \prime}$ $=\pi_{0}\left(\mathfrak{A}\left(\mathscr{D}_{q, \bar{p}}^{\prime}\right)\right)^{\prime \prime}=\pi_{0}\left(\mathfrak{A}\left(\mathscr{D}_{q, \bar{p}}\right)\right)^{\prime}=\pi_{0}\left(\mathfrak{A}\left(\mathscr{W}_{q, \bar{p}}\right)\right)^{\prime}=\pi_{0}\left(\mathfrak{A}\left(\mathscr{W}_{q, \bar{p}}^{\prime}\right)\right)^{\prime \prime}$.

A última expressão do Lema 4.5 é trivial para $A d S_{d}$, mas possui conseqüências potencialmente dramáticas para espaços-tempos AAdS não-triviais, pois, neste caso, $\pi_{0}\left(\mathfrak{A}\left(\mathscr{W}_{p, \bar{q}}\right)\right)^{\prime \prime} \cap \pi_{0}\left(\mathfrak{A}\left(\mathscr{W}_{\bar{p}, q}\right)\right)^{\prime \prime} \supset \pi_{0}\left(\mathfrak{A}\left(\mathscr{W}_{\bar{p}, q}^{\prime} \cap \mathscr{W}_{p, \bar{q}}^{\prime}\right)\right)^{\prime \prime}$, e a região $\mathscr{W}_{\bar{p}, q}^{\prime} \cap \mathscr{W}_{p, \bar{q}}^{\prime}$ é um aberto não-vazio se $(\mathscr{M}, g)$ satisfaz as hipóteses da Proposição 1.8 (página 21), mais fortes que as do Teorema 1.3 (ver Figura 1.4, página 22). Supondo que o pré-cofeixe de álgebras de von Neumann $\mathscr{K}(\mathscr{M}, g) \ni \mathscr{O} \mapsto \pi_{0}(\mathfrak{A}(\mathscr{O}))^{\prime \prime}$ satisfaz a propriedade de localidade estendida

$$
\pi_{0}\left(\mathfrak{A}\left(\mathscr{O}_{1}\right)\right)^{\prime \prime} \cap \pi_{0}\left(\mathfrak{A}\left(\mathscr{O}_{1}\right)\right)^{\prime \prime}=\mathbb{C} \mathbb{1}, \forall \mathscr{O}_{1}, \mathscr{O}_{2} \in \mathscr{K}(\mathscr{M}, g): \mathscr{O}_{1} \perp \mathscr{O}_{2}
$$

segue então que $\pi_{0}\left(\mathfrak{A}\left(\mathscr{W}_{\bar{p}, q}^{\prime} \cap \mathscr{W}_{p, \bar{q}}^{\prime}\right)\right)^{\prime \prime}=\mathbb{C} \mathbb{1}$ e, portanto, $\pi_{0}\left(\mathfrak{A}\left(\mathscr{O}_{r, s}\right)\right)^{\prime \prime}=\mathbb{C} \mathbb{1}$ para quaisquer $r, s \in \mathscr{M}$ tais que $\mathscr{O}_{p, q} \stackrel{p}{=} I^{+}(r, \mathscr{M}) \cap I^{-}(s, \mathscr{M}) \subset \mathscr{W}_{\bar{p}, q}^{\prime} \cap \mathscr{W}_{p, \bar{q}}^{\prime}$. Como:

- Qualquer diamante suficientemente pequeno em $(\mathscr{M}, g)$ satisfaz a condição acima para algum par $p, q \in \mathscr{I}$ satisfazendo as condições do Lema 4.5, e

- No espaço-tempo de Minkowski $\mathbb{R}^{1, d-1}$, qualquer teoria de observáveis locais aditiva, covariante por translações e localmente causal na representação GNS $\pi_{0}$ 
associada a um estado puro de vácuo (i.e., o espectro conjunto dos geradores de translações está contido em $J^{+}\left(0, \mathbb{R}^{1, d-1}\right)$ e o vetor de vácuo é um autovetor dos geradores com autovalores zero) satisfaz localidade estendida [Lan69],

somos levados ao seguinte "teorema "no-go":

Corolário 4.6 Sejam $(\mathscr{M}, g)$ um espaço-tempo AAdS satisfazendo as hipóteses da Proposição 1.8, $\mathfrak{A}$ uma teoria quântica localmente covariante estendida realizada em $(\mathscr{M}, g)$ e $\pi_{0}$ uma ${ }^{*}$-representação irredutivel de $\mathfrak{A}_{\mathscr{M}}$ satisfazendo as hipóteses do Lema 4.5. Se $\pi_{0}$ satisfaz localidade estendida (4.20), então qualquer álgebra de VON NEUMANN localizada em um aberto suficientemente pequeno consiste apenas em múltiplos de $\mathbb{1}$. Se o pré-cofeixe de álgebras de von Neumann $\mathscr{K}(\mathscr{M}, g) \ni \mathscr{O} \mapsto \pi_{0}(\mathfrak{A}(\mathscr{O}))^{\prime \prime}$ for, além disso, aditivo, então $\pi_{0}\left(\mathfrak{A}_{\mathscr{M}}\right)^{\prime \prime}=\mathbb{C} \mathbb{1}$.

OBSERVAÇÃo 4.13 Não existe, no conhecimento do Autor, uma demonstração da propriedade de localidade estendida em espaços-tempos curvos dentro de hipóteses similares às empregadas no espaço-tempo de Minkowski (i.e., mediante a substituição da condição espectral do vácuo por, digamos, alguma versão da condição de espectro microlocal [BFK96]). Conjeturamos, todavia, que tal demonstração é possível em espaços-tempos AAdS real-analíticos, em linhas similares à demonstração da propriedade do tubo tipo tempo de BORCHERS (página 83 e nota de rodapé 9).

Concluímos a partir do Corolário 4.6 que a dualidade de HAAG essencial com respeito a $\mathscr{D}(\mathscr{M}, g)$ implicam potencialmente numa teoria quântica trivial se combinadas com aditividade no interior, e mesmo uma teoria não-aditiva no interior pode acabar sendo trivial em escalas suficientemente pequenas - em particular, o procedimento de reconstrução holográfica da realização de $\mathfrak{A} \mathrm{em}(\mathscr{M}, g)$ a partir da teoria dual na fronteira, cuja parte geométrica foi esquematizada na Subsubseção 1.3.1.3 (página 24), é inútil em ambos os casos! ${ }^{12}$ A seguinte hipótese, contudo, leva a resultados não-triviais:

(v) $\pi_{0}$ é essencialmente HAAG-dual com respeito a $\mathscr{W}(\mathscr{M}, g)$, mas não é (essencialmente) HAAG-dual com respeito a $\mathscr{D}(\mathscr{M}, g)$.

\footnotetext{
${ }^{12}$ Por outro lado, a possibilidade de que $\pi_{0}$ seja HAAG-dual e $\pi_{0}\left(\mathfrak{A}\left(\mathscr{W}_{p, \bar{q}}^{\prime} \cap \mathscr{W}_{q, \bar{p}}^{\prime}\right)\right)^{\prime \prime}=\mathbb{C} \mathbb{1}$ não deixa de ser interessante do ponto de vista físico - como cunhas modelam, num certo sentido, o exterior de buracos negros, podemos imaginar a região $\mathscr{W}_{p, \bar{q}}^{\prime} \cap \mathscr{W}_{q, \bar{p}}^{\prime}$ como "dentro do horizonte". Desse ponto de vista, vemos que uma teoria quântica com as características acima é incapaz de "enxergar" eventos físicos localizados "dentro do horizonte de eventos". Como dualidade de HAAG com respeito a $\mathscr{D}(\mathscr{M}, g)$ é necessária para que a teoria quântica REHREN-dual na fronteira seja conformalmente covariante e que $\pi_{0}$ seja a representação GNS associada a um estado de vácuo [BGL93], conjeturamos que tal teoria quântica (não-aditiva) provavelmente não sofre do paradoxo de informação de buracos negros, mas é também essencialmente insensível aos detalhes da geometria no interior, da mesma forma que o limite de escala construído na Seção anterior.
} 
A segunda premissa nos permite escapar do Corolário 4.6. Em particular, neste caso o pré-cofeixe de álgebras de von Neumann $\mathscr{D}(\mathscr{M}, g) \ni \mathscr{D}_{p, \bar{q}} \mapsto \pi_{0}\left(\mathfrak{A}\left(\mathscr{D}_{q, \bar{p}}\right)\right)^{\prime}$ não é localmente causal, mas o pré-cofeixe

$$
\mathscr{W}(\mathscr{M}, g) \ni \mathscr{W}_{p, q} \mapsto \mathfrak{B}\left(\mathscr{W}_{p, q}\right) \doteq \pi_{0}\left(\mathfrak{A}\left(\mathscr{W}_{p, q}^{\prime}\right)\right)^{\prime}
$$

o é, pela hipótese (v).

LEMA 4.7 Suponha que $\pi_{0}$ satisfaz a hipótese (v). Então, $\pi_{0}$ satisfaz definiteza local holográfica se e somente se as álgebras quase-locais $\mathfrak{B}_{\mathscr{D} \text { oi(r) }}^{\prime \prime}$ são irredutíveis, i.e. $\mathfrak{B}_{\mathscr{P} \text { oi }(r)}^{\prime}=\mathbb{C} \mathbb{1}$, para todo $r \in \mathscr{I}$. Em particular, neste caso $\mathfrak{B}_{\mathscr{M}}^{\prime \prime}$ é irredutivel.

Prova. Segue imediatamente da fórmula

$$
\begin{gathered}
\bigcap_{\substack{(p, \bar{q}) \in \mathscr{D}(\mathscr{I}), \mathscr{W}_{p, \bar{q} \ni \bar{r}}}} \pi_{0}\left(\mathfrak{A}\left(\mathscr{W}_{p, \bar{q}}\right)\right)^{\prime \prime}=\bigcap_{\substack{(p, \bar{q}) \in \mathscr{D}(\mathscr{T}), \mathscr{W}_{p, \bar{q} \ni \bar{r}}}} \pi_{0}\left(\mathfrak{A}\left(\mathscr{W}_{q, \bar{p}}^{\prime}\right)\right)^{\prime \prime}= \\
=\left(\bigcup_{q \ll \mathscr{I} \bar{p} \in \mathscr{M} i n(r)} \mathfrak{B}\left(\mathscr{W}_{q, \bar{p}}\right)\right)^{\prime},
\end{gathered}
$$

e do fato de que

$$
\bigcap_{\substack{(p, \bar{q}) \in \mathscr{D}(\mathscr{F}), \mathscr{W}_{p}, \bar{q} \ni \bar{r}}} \mathscr{W}_{q, \bar{p}}^{\prime}=\bigcap_{\substack{(p, \bar{q}) \in \mathscr{D}(\mathscr{F}), \mathscr{W}_{p, \bar{q}} \ni \bar{r}}} \mathscr{W}_{p, \bar{q}}=\{\bar{r}\},
$$

em virtude da Proposição 1.8 e do Teorema 1.3.

Em particular, simetrias internas do pré-cofeixe $\mathscr{W}_{p, q} \mapsto \mathfrak{B}\left(\mathscr{W}_{p, q}\right)$ não podem estar espontaneamente quebradas em $\mathscr{H}_{0}$ se $\pi_{0}$ satisfaz definiteza local holográfica e a hipótese $(\mathrm{v})$. Note que isto não implica que as simetrias internas do pré-cofeixe $\mathscr{W}_{p, q} \mapsto \pi_{0}\left(\mathfrak{A}\left(\mathscr{W}_{p, q}\right)\right)^{\prime \prime}$ não estejam quebradas.

Consideremos, agora, uma ${ }^{*}$-representação $\pi$ de $\mathfrak{A}_{\mathscr{M}}$ no espaço de HilBERT $\mathscr{H}_{\pi}$ satisfazendo

$\forall p, q \in \mathscr{D}(\mathscr{I}), \exists V \in \mathscr{B}\left(\mathscr{H}_{0}, \mathscr{H}_{\pi}\right)$ unitário tal que $\pi(A)=V \pi_{0}(A) V^{*}, \forall A \in \mathfrak{A}\left(\mathscr{W}_{p, q}^{\prime}\right)$.

Em particular, $\pi$ satisfaz o critério DHR com respeito a $\mathscr{D}(\mathscr{M}, g)$ mediante a bijeção de Rehren. O fato de que $\pi_{0}$ não satisfaz dualidade de HAAG essencial com respeito a $\mathscr{D}(\mathscr{M}, g)$ garante que as *-representações satisfazendo (4.22) constituem uma subclasse própria de excitações DHR.

Convém notar que, mesmo do ponto de vista da forma original da dualidade de ReHREN, i.e., $(\mathscr{M}, g)=A d S_{d}$, parece estranho à primeira vista empregar um critério 
de seleção que visa modelar excitações do vácuo correspondendo a partículas, uma vez que teorias quânticas de campo conformes não são teorias de partículas elementares. Entretanto, foi demonstrado por BuchHolz, Mack e Todorov [BMT88] que:

TEOREMA 4.8 ([BMT88]) Se $\pi_{0}$ é a representação GNS de um estado de vácuo $\omega_{0}$ conformalmente invariante (e que, portanto, é HAAG-dual com respeito a $\mathscr{D}\left(A d S_{d}\right)$ [BGL93]), qualquer estado $\omega$ com representação GNS $\pi_{\omega}$ de energia positiva é uma excitação DHR de $\omega_{0}$, i.e., dado qualquer $(p, q) \in \mathscr{D}(\mathscr{I})$, temos que $\pi_{\omega}\left\lceil_{\mathfrak{A}(\mathscr{D} p, q}\right)$ é unitariamente equivalente a $\pi_{0} \Upsilon_{\mathfrak{A}\left(\mathscr{D}_{p, q}^{\prime}\right)}$.

Prova. $\quad$ Considere os seguintes fatos: (i) $(p, \bar{q}) \in \mathscr{D}(\mathscr{I})$ se e somente se $(q, \bar{p}) \in$ $\mathscr{D}(\mathscr{I})$; (ii) $\omega_{0} \uparrow_{\pi_{0}\left(\mathfrak{A}\left(\mathscr{D}_{p, q}\right)\right)^{\prime \prime}}$ é normal e fiel para todo $(p, q) \in \mathscr{D}(\mathscr{I})$ (propriedade de ReEH-Schlieder). Como a propriedade de REeH-Schlieder também vale se substituirmos $\pi_{0}$ por $\pi$ em virtude da positividade da energia-momento, segue que a aplicação $\pi_{0}(A) \mapsto \pi(A)$ para $A \in \mathfrak{A}\left(\mathscr{D}_{p, q}\right)$ se estende a um *-isomorfismo normal entre $\pi_{0}\left(\mathfrak{A}\left(\mathscr{D}_{p, q}\right)\right)^{\prime \prime}$ e $\pi_{\omega}\left(\mathfrak{A}\left(\mathscr{D}_{p, q}\right)\right)^{\prime \prime}$. Como os vetores cíclicos $\Omega_{0}$ e $\Omega$ nos respectivos espaços de Hilbert GNS $\mathscr{H}_{0}$ e $\mathscr{H}_{\omega}$ associados a $\omega_{0}$ e $\omega$ são também separantes pela propriedade de REEH-SCHLIEDER, segue do Teorema 2.5.32 em [BR87] (ver também o Teorema 7.2.9 em [KR86]) que tal *-isomorfismo é implementado por um operador unitário entre os espaços de Hilbert GNS de $\omega_{0}$ e $\omega$. A partir de (i), vemos finalmente que $\pi$ satisfaz o critério DHR, com $\rho$ localizado no diamante $\mathscr{D}_{p, q}^{\prime}$

Como a propriedade de REEH-SCHLIEDER é válida para as representações de referência $\pi_{0}$ que adotamos para espaços-tempos AAdS, vemos que tal critério é suficientemente geral para nossos propósitos. O endomorfismo $\rho$ correspondente a $\pi$ e localizado, digamos, em $\mathscr{D}_{p, \bar{q}}$ possui as seguintes propriedades:

1. Por construção, $\rho \circ \pi_{0}(A)=\pi_{0}(A)$ para todo $A \in \mathfrak{A}\left(\mathscr{D}_{q, \bar{p}}\right)$;

2. Se $\left(p^{\prime}, q^{\prime}\right) \in \mathscr{D}(\mathscr{I})$ são tais que $p, \bar{q}, p^{\prime}, q^{\prime} \in \mathscr{M}$ in $(r)$ para algum $r \in \mathscr{I}$ e $B \in$ $\mathfrak{A}\left(\mathscr{D}_{p^{\prime}, q^{\prime}}\right)$, então $\rho \circ \pi_{0}(B) \in \mathfrak{B}\left(\mathscr{D}_{p^{\prime \prime}, q^{\prime \prime}}\right)$ para $\left(p^{\prime \prime}, q^{\prime \prime}\right) \in \mathscr{D}(\mathscr{I})$ tal que $\mathscr{D}_{p, \bar{q}}, \mathscr{D}_{p^{\prime}, q^{\prime}} \subset$ $\mathscr{D}_{p^{\prime \prime}, q^{\prime \prime}}$ (repetir o argumento usado na Subseção 4.4.1, página 88).

A segunda propriedade acima leva ao seguinte cenário: $\rho \circ \pi_{0}$ só assume valores em

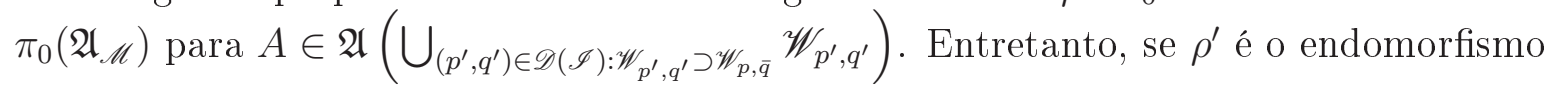
associado a $\pi$ e localizado em $\mathscr{W}_{q, \bar{p}}$, temos que $\rho^{\prime}$ ainda pode ser obtido pelo transporte de $\rho$ por meio de "intertwiners" locais, pois, embora não exista $\left(p^{\prime}, q^{\prime}\right) \in \mathscr{D}(\mathscr{I})$ tal que $\mathscr{W}_{p^{\prime}, q^{\prime}} \supset \mathscr{W}_{p, \bar{q}} \cup \mathscr{W}_{q, \bar{p}}, \rho$ sempre pode ser transportado para uma cunha menor contida em $\mathscr{W}_{q, \bar{p}}$. 
Podemos imaginar, em vista da possibilidade da quebra espontânea de simetrias internas permitida pela dualidade de HAAG essencial, que $\pi_{0}$ não é irredutível. Mais precisamente, suponhamos que o endomorfismo (trivialmente) localizado $\iota \doteq \mathrm{id}_{\pi_{0}\left(\mathfrak{A}_{\mathscr{M}}\right)}$ associado à $\pi_{0}$ possui subobjetos não-triviais, i.e., para cada $(p, \bar{q}) \in \mathscr{D}(\mathscr{I})$ existe uma projeção $\mathbb{1} \neq E \in \pi_{0}\left(\mathfrak{A}\left(\mathscr{W}_{p, \bar{q}}\right)\right)^{\prime}$, um *-endomorfismo $\sigma$ de $\pi_{0}\left(\mathfrak{A}_{\mathscr{M}}\right)$ localizado em $\mathscr{W}_{p, \bar{q}}$ e uma isometria $W \in(\sigma, \iota)$ tal que $W W^{*}=E$ (pela propriedade B de Borchers, $\left.W \in \pi_{0}\left(\mathfrak{A}\left(\mathscr{W}_{p, \bar{q}}\right)\right)^{\prime}\right)$. Na verdade, só precisamos da existência de $E$ (garantida, por sua vez, pela não-irredutibilidade de $\pi_{0}$ ), pois a existência de $W$ e, portanto, de $\sigma$ $\left(\sigma\left(\pi_{0}(A)\right) \doteq W^{*} \pi_{0}(A) W\right)$ segue da propriedade B. Assim, podemos considerar também a isometria $V$ e o endomorfismo localizado $\sigma^{\prime}$ associados à projeção $\mathbb{1}-E$. Tanto $\sigma \circ \pi_{0}$ como $\sigma^{\prime} \circ \pi_{0}$ satisfazem dualidade de HAAG essencial com respeito a $\mathscr{W}(\mathscr{M}, g)$.

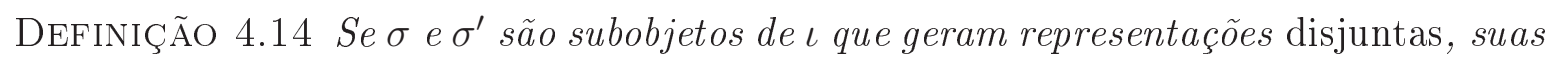
classes de equivalência unitária são então denominadas fases do estado de referência $\omega_{0}$. Uma fase $\sigma$ é dita pura se a representação correspondente for irredutivel.

Tomemos os subobjetos $\sigma$ e $\sigma^{\prime}$ de $\iota$ construídos no parágrafo anterior localizados respectivamente, digamos, em $\mathscr{D}_{p, \bar{q}}$ e $\mathscr{D}_{q, \bar{p}}$ Como neste caso $\sigma\left(\pi_{0}(A)\right)=\sigma^{\prime}\left(\pi_{0}(A)\right)=$ $\pi_{0}(A)$ se $A \in \mathfrak{A}\left(\mathscr{W}_{p, \bar{q}}^{\prime}\right) \cap \mathfrak{A}\left(\mathscr{W}_{q, \bar{p}}^{\prime}\right) \supset \mathfrak{A}\left(\mathscr{W}_{p, \bar{q}}^{\prime} \cap \mathscr{W}_{q, \bar{p}}^{\prime}\right)$, podemos considerar então o seguinte *-endomorfismo $\tau$ de $\pi_{0}\left(\mathfrak{A}_{\mathscr{M}}\right)^{\prime \prime}$, que satisfaz as seguintes propriedades:

(S1) $\tau \circ \sigma\left(\pi_{0}(A)\right)=\sigma\left(\pi_{0}(A)\right)$ para todo $A \in \mathfrak{A}\left(\mathscr{W}_{p, \bar{q}}\right)$;

(S2) $\tau \circ \sigma^{\prime}\left(\pi_{0}(A)\right)=\sigma^{\prime}\left(\pi_{0}(A)\right)$ para todo $A \in \mathfrak{A}\left(\mathscr{W}_{q, \bar{p}}\right)$.

Obviamente, temos que $\tau \circ \sigma\left(\pi_{0}(A)\right)=\tau \circ \sigma^{\prime}\left(\pi_{0}(A)\right)=\tau\left(\pi_{0}(A)\right)$ se $A \in \mathfrak{A}\left(\mathscr{W}_{p, \bar{q}}^{\prime} \cap\right.$ $\left.\mathscr{W}_{q, \bar{p}}^{\prime}\right)$. Podemos então dizer que $\tau$ está "localizado" em $\mathscr{W}_{p, \bar{q}}^{\prime} \cap \mathscr{W}_{q, \bar{p}}^{\prime}$ e interpola as fases $\sigma$ e $\sigma^{\prime}$ (lembremos que, em espaços-tempos AAdS satisfazendo, como assumimos nesta Seção, as hipóteses da Proposição 1.8 e, portanto, do Teorema 1.3, $\mathscr{W}_{p, \bar{q}}^{\prime} \cap \mathscr{W}_{q, \bar{p}}^{\prime} \neq \varnothing$ ! Ver Figura 1.4, página 1.4).

DEFINIÇÃO 4.15 Um endomorfismo $\tau$ que satisfaz (S1) e (S2) para algum par de fases $\sigma, \sigma^{\prime}$ de $\omega_{0}$ localizadas respectivamente em cunhas $\mathscr{W}_{p, \bar{q}}, \mathscr{W}_{q, \bar{p}}$ é dito solitônico. Dizemos então que $\tau$ separa ou interpola $\sigma$ e $\sigma^{\prime}$, e que a classe de equivalência unitária de $\tau$ é um sóliton.

Note que um "intertwiner" que transporte $\sigma$ da cunha $\mathscr{W}_{p, \bar{q}}$ a uma cunha suficientemente próxima $\mathscr{W}_{p^{\prime}, \overline{q^{\prime}}}$, quando aplicado a $\sigma^{\prime}$, transporta-o de $\mathscr{W}_{q, \bar{p}}$ a $\mathscr{W}_{q^{\prime}, \overline{p^{\prime}}}$ e, portanto, quando aplicado a $\tau$, produz um endomorfismo solitônico "localizado" em $\mathscr{W}_{p^{\prime}, \overline{q^{\prime}}}^{\prime} \cap \mathscr{W}_{q^{\prime}, \overline{p^{\prime}}}^{\prime}$ no sentido acima. Portanto, é-nos lícito remover as aspas e dizer que $\tau$ é, de fato, localizado em $\mathscr{W}_{p, \bar{q}}^{\prime} \cap \mathscr{W}_{q, \bar{p}}^{\prime}$. A composição de endomorfismos solitônicos $\tau, \tau^{\prime}$ não é sempre definida, pois exige que os endomorfismos correspondentes representando as fases interpoladas por $\tau$ e $\tau^{\prime}$ "se encaixem". 


\subsubsection{Obstruções à inversão da dualidade de REHREN}

Resta discutir a possibilidade de, partindo de um pré-cofeixe $\mathscr{D}_{p, q} \mapsto \mathfrak{A}\left(\mathscr{D}_{p, q}\right)$ realizando uma teoria quântica localmente covariante $\mathfrak{A}$ em $\left(\mathscr{I}, \bar{g}^{(0)}\right)$, construirmos para cada espaço-tempo AAdS $(\mathscr{M}, g)$ satisfazendo as hipóteses da presente Seção um précofeixe $\mathscr{O} \mapsto \mathfrak{A}(\mathscr{O})$ indexado pelas regiões abertas, globalmente hiperbólicas e causalmente convexas $\mathscr{O} \subset \mathscr{M}$, e não apenas cunhas (cujas álgebras locais são obtidas definindo $\left.\mathfrak{A}\left(\mathscr{W}_{p, q}\right) \doteq \mathfrak{A} \circ \rho_{(\mathscr{M}, g)}\left(\mathscr{W}_{p, q}\right)\right)$.

Tomemos um estado de referência $\omega$ na realização de $\mathfrak{A}$ em $(\mathscr{M}, g)$. Se o précofeixe de álgebras de VON NeUmann $\mathscr{O} \mapsto \pi_{\omega}(\mathfrak{A}(\mathscr{O}))^{\prime \prime}$ for aditivo, podemos tentar fazer essa reconstrução empregando os resultados da Subsubseção 1.3.1.3 (página 24) se definirmos, para um diamante relativamente compacto e causalmente convexo $\mathscr{O}_{p, q} \subset$ $\mathscr{M}$ suficientemente pequeno,

$$
\mathfrak{B}\left(\mathscr{O}_{p, q}\right) \doteq \bigcap_{\mathscr{W}_{p, q} \supset \mathscr{O}_{p, q}} \pi_{\omega}\left(\mathfrak{A}\left(\mathscr{W}_{p, q}\right)\right)^{\prime \prime}
$$

Proposição 4.9 Suponha que o pré-cofeixe de álgebras de vOn Neumann $\mathscr{O} \mapsto$ $\pi_{\omega}(\mathfrak{A}(\mathscr{O}))^{\prime \prime}$ é aditivo e satisfaz dualidade de HAAG para cunhas. Então $\mathfrak{B}\left(\mathscr{O}_{p, q}\right)=$ $\pi_{\omega}\left(\mathfrak{A}\left(\mathscr{O}_{p, q}^{\prime}\right)\right)^{\prime}$.

Prova. Notemos que a operação que leva uma álgebra de von Neumann no seu comutante satisfaz as mesmas propriedades que a operação de complemento causal em regiões causalmente completas (ver Apêndice A, final da Subseção A.2.1, página 119), se substituirmos a união de álgebras pela álgebra gerada pela união. Segue então de (4.23) que $\mathfrak{B}\left(\mathscr{O}_{p, q}\right) \doteq \bigcap_{\mathscr{W}_{p, q} \supset \mathscr{O}_{p, q}} \pi_{\omega}\left(\mathfrak{A}\left(\mathscr{W}_{p, q}^{\prime}\right)\right)^{\prime}=$ $\left(\bigvee_{\mathscr{W}_{p, q} \supset \mathscr{O}_{p, q}} \pi_{\omega}\left(\mathfrak{A}\left(\mathscr{W}_{p, q}\right)^{\prime}\right)^{\prime \prime}\right)^{\prime}=\pi_{\omega}\left(\mathfrak{A}\left(\bigcup_{\mathscr{W}_{p, q} \supset \mathscr{O}_{p, q}} \mathscr{W}_{p, q}^{\prime}\right)\right)^{\prime}=\pi_{\omega}\left(\mathfrak{A}\left(\mathscr{O}_{p, q}^{\prime}\right)\right)^{\prime}$

Se o pré-cofeixe $\mathscr{O} \mapsto \pi_{\omega}(\mathfrak{A}(\mathscr{O}))^{\prime \prime}$ for HAAG-dual, então é também aditivo e, assim, o procedimento de reconstrução baseado em (4.23) pode ser conduzido até o fim de maneira bem sucedida. Se assumirmos, por outro lado, apenas dualidade de HAAG essencial, o pré-cofeixe HAAG-dual $\mathscr{O} \mapsto \pi_{\omega}\left(\mathfrak{A}\left(\mathscr{O}^{\prime}\right)\right)^{\prime}$ não precisa ser aditivo. Tendo em vista o cenário de quebra espontânea de simetrias internas, podemos dar uma interpretação física natural para este fenômeno: a rede HAAG-dual contorna a quebra espontânea de simetrias internas incluindo precisamente observáveis correspondendo a procedimentos físicos estendidos, localizados, por exemplo, ao redor de paredes de domínio, tal qual os setores solitônicos construídos na Subseção anterior. Tal situação é análoga a fases ordenadas em modelos de spin quânticos em Mecânica Estatística, onde a dualidade de KRAMERS-WANNIER produz um gás de "contornos ao redor de domínios de fase pura" que, por sua vez, encontra-se na fase simétrica (desordenada) [Kad00, Min00]. Se imaginarmos a região $\mathscr{W}_{p, \bar{q}}^{\prime} \cap \mathscr{W}_{q, \bar{p}}^{\prime}(\neq \varnothing !)$, esboçada na Figura 1.4 
(página 1.4) como a localização aproximada de uma parede de dominio, i.e., uma subvariedade de $\mathscr{M}$ maximal (ou seja, que não pode ser mergulhada em nenhuma outra subvariedade de mesma codimensão) e acausal de codimensão 2, é bastante tentador interpretar os sólitons na Definição 4.15 à luz da correspondência AdS/CFT como $D$ branas, pois, como vimos, estes compartilham essencialmente as mesmas propriedades dos objetos de mesmo nome em Teoria de Cordas [Zwi04].

Notamos ademais que setores de superseleção induzidos gravitacionalmente já foram considerados anteriormente na literatura, em particular com relação à regra de superseleção de carga elétrica [AS80, Sor79]. Tais setores gozam de razoável universalidade, e são induzidos até mesmo em teorias quânticas de campo livres.

Enfatizamos que os setores solitônicos que construímos não existem em $A d S_{d}$, pois neste caso dualidade de HAAG e dualidade de HAAG essencial em relação a $\mathscr{W}\left((\mathscr{M}, g)=A d S_{d}\right)$, como vimos, coincidem (isto, é claro, é diretamente relacionado ao fato de que $\left.\mathscr{W}_{p, \bar{q}}^{\prime} \cap \mathscr{W}_{q, \bar{p}}^{\prime}=\varnothing \mathrm{em} A d S_{d}\right)$, e a soma direta de ${ }^{*}$-representações de referência satisfazendo dualidade de HAAG não necessariamente também o faz, mas a soma direta de *-representações de referência essencialmente HAAG-duais é essencialmente HAAG-dual [Rob04]. Mais em geral, a dualidade de HAAG para cunhas leva, pelo Lema 4.7, à irredutibilidade de $\pi_{0}$, impossibilitando a existência de setores solitônicos mesmo se conseguirmos escapar do Corolário 4.6 e ter observáveis não triviais para $\mathscr{O} \subset \mathscr{M}$ arbitrariamente pequeno para $(\mathscr{M}, g) \neq A d S_{d}$ (em $A d S_{d}$, não há problema em assumir dualidade de HAAG, e neste caso a dualidade de REHREN original permite uma correspondência biunívoca). Assim, a presença de setores solitônicos parece essencialmente inutilizar ou impossibilitar (modulo a validade da propriedade de localidade estendida) a reconstrução inequívoca da teoria quântica no interior por meio da prescrição (4.23) em geometrias AAdS não-triviais. Especulamos que uma possibilidade alternativa, mais indireta, para uma reconstrução pelo menos parcial, mas além da mera especificação da localização dos observáveis em cunhas dada pela bijeção de REHREN, seria elaborar uma expansão de produto de operadores ao redor do limite de escala, tal qual sugerido no final da Seção 4.3 . 


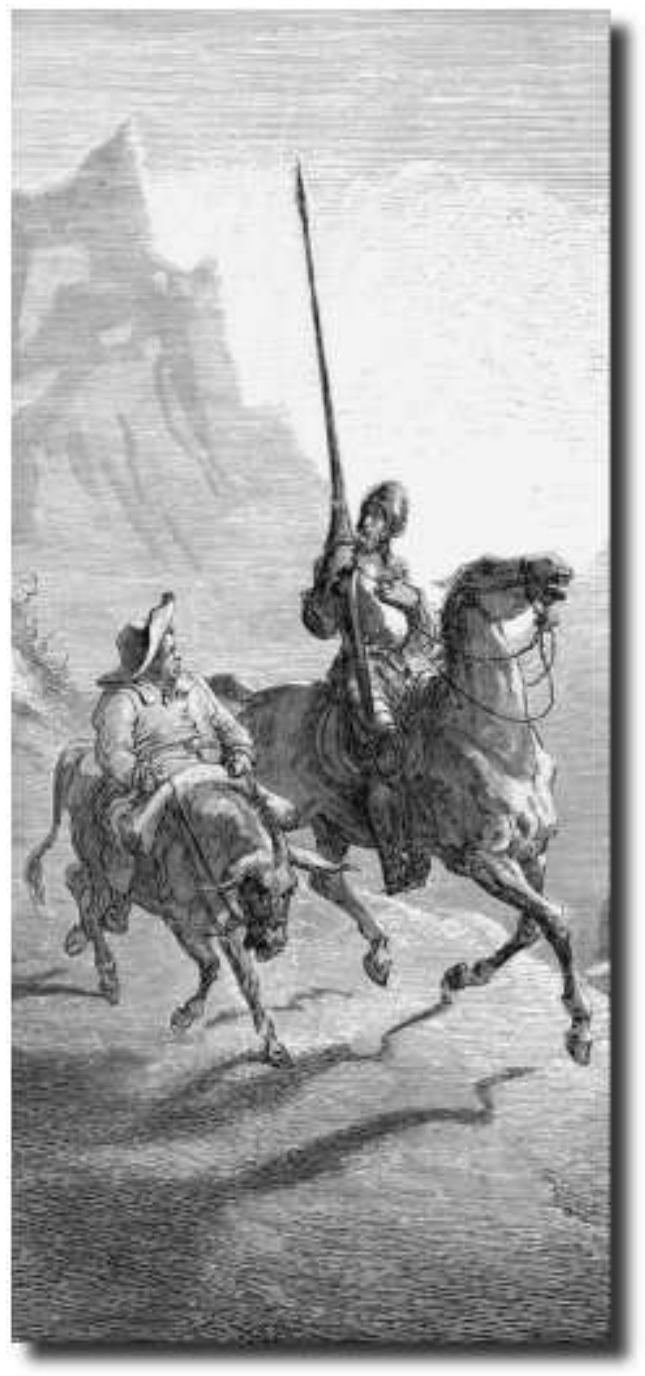

- ¿Cómo puede ser eso? - respondió don Quijote - ¿ ¿Tan de esencia de la historia es saber las cabras que han pasado por estenso, que si se yerra una del número no puedes seguir adelante con la historia?

- No, señor, en ninguna manera respondió Sancho -; porque así como yo pregunté a vuestra merced que me dijeste cuántas cabras habían pasado, y me respondió que no sabía, en aquel mesmo instante se me fue a mí de la memoria cuanto que quedaba por decir, y a fe que era de mucha virtud y contiento.

- ¿De modo - dijo don Quijote - que ya la historia es acabada?

- Tan acabada es como mi madre - dijo Sancho.

- Dígote de verdad - respondió don Quijote - que tú has contado unas de las más nuevas consejas, cuento o historia que nadie pudo pensar en el mundo, y que tal modo de contarla ni dejarla jamás se podrá ver ni habrá visto en toda la vida, aunque no esperaba yo otra cosa de tu buen discurso; mas no me maravillo, ques quizá estos golpes que no cesan te deben de tener turbado el entendimiento. ${ }^{\mathrm{xvi}}$

Miguel de Cervantes SaAvedra El ingenioso hidalgo don Quijote de la Mancha, Primero Libro, Cap. XX 



\section{Coda. Considerações finais}

Go, go, go, said the bird: human kind

Cannot bear very much reality.

Time past and time future

What might have been and what has been

Point to one end, which is always

present. ${ }^{\text {xvii }}$

T. S. ELIOT

"Burnt Norton" (Four Quartets $\left.{ }^{\mathrm{xviii}}, 1943\right)$, I

O presente trabalho foi capaz de revelar uma estrutura geral bastante rica subjazendo à correspondência $\mathrm{AdS} / \mathrm{CFT}$, da qual fomos capazes apenas de arranhar a superfície.

As principais contribuições do presente trabalho, como um todo, podem ser listadas a seguir:

- Foram especificados os aspectos essenciais subjacentes à dualidade de REHREN. Em particular, o caráter geométrico e causal da mudança de localização proposta por REHREN foi delineado tanto do ponto de vista global (Capítulo 1) como local (i.e., numa vizinhança de $\mathscr{I}$ - Capítulo 2).

- O elaborado maquinário de geometria Lorentziana global desenvolvido a partir de dois diferentes pontos de vista - cinemático (estrutura causal, infinito conforme) e dinâmico (evolução temporal, retorno ao equilíbrio) - permitiu determinar condições suficientemente robustas sobre a geometria dos espaços-tempos AAdS tais que certas propriedades básicas da dualidade de REHREN são preservadas e outras são modificadas de forma relativamente clara, conforme visto no Teorema 1.3 (página 17), na Proposição 1.8 (página 21) e no Teorema 1.14 (página 28).

- Propusemos uma maneira geometricamente intrínseca de construir funções tempo globais associadas a diamantes relativamente compactos em espaços-tempos cau- 
salmente simples e cunhas AAdS, que admite uma formulação das leis zero (assintótica) e segunda da dinâmica de buracos negros (Seção 2.3, página 40ff.).

- Em nível quântico, implementamos uma instância do princípio de covariância local, proposto por Brunetti, Fredenhagen e Verch (Capítulo 3), numa situação que demanda a imposição de condições de contorno (ver Definição 4.1, página 72, e a discussão que se segue) adaptadas à geometria de espaços-tempos (A)AdS, com a ajuda do formalismo algébrico desenvolvido por SOMMER e do caso-protótipo de $A d S_{d}$ (Proposição 4.4, página 80).

- Propomos um formalismo de "álgebras de escala" ao redor de pontos no infinito conforme de espaços-tempos AAdS que, auxiliado pelos poderosos resultados estruturais de Borchers e YNGVAson (Teoremas (4.1) a (4.3), página 77ff.) e pelo ponto de vista geométrico da Seção 2.3, não só permitem uma determinação precisa de condições de contorno para estados fisicamente relevantes, como também estabelecem uma estreita relação entre retorno ao equilíbrio térmico pela ação de isometrias assintóticas e limites de escala (condição (d), página 86 e a discussão que se segue).

- Indicamos como excitações solitônicas similares a D-branas (Definição 4.15, página 96) podem surgir no caso de geometrias AAdS não-triviais, relacionando-as a uma estrutura não trivial de vácuo (Definição 4.14, página 96) e a uma potencial impossibilidade de reconstruir a teoria quântica no interior a partir de seu dual de REHREN por meio da prescrição (4.23) (página 97).

Um problema de grande interesse na literatura de Geometria Lorentziana concerne a estabilidade e rigidez de espaços-tempos AAdS mediante condições similares às assumidas no Teorema 1.3 e na Proposição 1.8. Um importante resultado parcial foi obtido recentemente por ANDERSON [And06b], que nos diz que, dados (i) uma função tempo global $t$ num espaço-tempo AAdS $(\mathscr{M}, g)$ assintoticamente simples, geodesicamente completo e satisfazendo uma condição técnica de unicidade de continuação de soluções das equações de Einstein linearizadas através do infinito conforme, (ii) uma seqüência de dados de CAUCHY definidos em níveis distintos $t^{-1}\left(t_{i}\right)$ de $t$, tais que $t_{i} \stackrel{i \rightarrow \infty}{\longrightarrow}+\infty$, e que tendem a dados de CAUCHY estacionários, então $(\mathscr{M}, g)$ é globalmente estacionário. Embora este não seja um resultado estrito de rigidez, ilustra como as condições de contorno no infinito conforme atuam para impedir a dispersão de perturbações nos dados de CAuchy. Estamos, contudo, longe de um resultado acerca da estabilidade não-linear global de espaços-tempos (A)AdS com a mesma precisão que o de CHRIStodoulou e Klainerman [CK93] para o espaço-tempo de Minkowski (ver também a Observação 2.2, página 53, para mais considerações a respeito). Outra questão em aberto é a factibilidade de se demonstrar a rigidez de espaços-tempos AdS mediante ausência de atraso temporal gravitacional de geodésicas nulas [PSW02] (Ver Figura 1.3, 
página 17). Tais questões nos interessam na medida em que respondê-las nos diria quão naturais são nossas hipóteses geométricas do ponto de vista gravitacional.

A construção de funções tempo globais para diamantes relativamente compactos que obtivemos podem, acreditamos, ser ainda mais aprofundadas, na direção de análogos assintóticos das outras leis da dinâmica de buracos negros, que nos dariam mais detalhes sobre as "flutuações" de quantidades geométricas ao redor do limite de escala nas extremidades dos diamantes, buscando assim um análogo d(e uma generalização d)a primeira lei da dinâmica de buracos negros para diamantes. Do ponto de vista das cunhas AAdS, interessa-nos relacionar alguma quantidade deste tipo com a parte elétrica rescalonada $E_{a b}$ do tensor de WEYL, que entra como parte dos "dados iniciais" para a expansão de FEFFERMAN-GRAHAM da métrica ao redor do infinito.

A limitação mais gritante do presente trabalho é a total ausência de exemplos nãotriviais, tanto no que concerne a geometria como modelos (mesmo livres) em Teoria Quântica de Campos. A questão geométrica, como vimos acima, envolve encontrar exemplos não triviais de espaços-tempos AAdS que não sejam singulares, o que é muito difícil sem um resultado geral como no caso de constante cosmológica zero. Em relação à parte quântica, uma direção futura de investigação que é bastante convidativa é checar a condição de contorno (4.17), pelo menos para campos livres, em termos de premissas mais familiares. Fazer isto em espaços-tempos gerais envolve mergulhar no arsenal de análise microlocal [Hör71, DH72, Dui96, Hör90] para construirmos de maneira precisa as funções de GREEN.

A construção de uma expansão de produto de operadores dentro de nossa extensão da dualidade de REHREN representa um desafio à parte, mas do mais alto interesse, pois pode potencialmente revelar relações entre desvios do equilíbrio térmico em cunhas AAdS com quantidades geométricas tais como $E_{a b}$. Um exemplo que motiva tal conjectura é a relação entre os pólos das funções de dois pontos retardadas da teoria quântica dual na fronteira, que determinam as propriedades de retorno ao equilíbrio, e modos quase-normais em buracos negros AAdS [HH00, SS02]. Tal relação deve possuir um análogo para cunhas e campos livres em AAdS.

Por fim, obviamente apresentamos na Subseção 4.4 .2 (página 91ff.) apenas o mais básico das propriedades dos setores solitônicos na teoria quântica no interior associados a pares de fases (satisfazendo o critério DHR) da sua teoria REHREN-dual na fronteira. Os resultados apresentados acima certamente merecem um escrutínio mais profundo, nas linhas dos trabalhos de Müger [Müg98, Müg96] e Schlingemann [Sch96] no caso geral, e nas linhas dos trabalhos de ASHTEkAR e SEn [AS80] e Sorkin [Sor79] na construção de exemplos, combinando as técnicas destes artigos com o ferramental de análise microlocal supracitado. 

APÊNDICES 



\section{APÊNDICE A}

\section{Elementos de geometria Lorentziana}

Apresentaremos aqui as definições necessárias e o enunciado de alguns resultados úteis em geometria Lorentziana. Nossas referências são [BEE96, HE73, O’N83, Wal84].

\section{A.1 Teoria local}

Seja $\mathscr{M}$ uma variedade $d$-dimensional, $d \geq 2$. Uma métrica semi-Riemanniana de índice $p$ é uma seção $g$ de $T^{*} \mathscr{M} \otimes T^{*} \mathscr{M}$ tal que $g(r)$ é uma forma bilinear simétrica não-degenerada de índice $p, \forall r \in \mathscr{M}$. O par $(\mathscr{M}, g)$ é dito ser uma variedade semiRiemanniana - para $p=0,(\mathscr{M}, g)$ é simplesmente uma variedade Riemanniana. Se $p=1$, dizemos que $(\mathscr{M}, g)$ é uma variedade Lorentziana.

Em todos os casos, denotaremos por $\nabla_{a}$ a conexão de LEvi-Civita associada a $g$ - lembrar que uma conexão (de Koszul) num fibrado vetorial $\mathscr{E} \stackrel{p}{\longrightarrow} \mathscr{M}$ é uma aplicação $\nabla: \Gamma^{\infty}(\mathscr{M}, T \mathscr{M}) \times \Gamma^{\infty}(\mathscr{M}, \mathscr{E}) \rightarrow \Gamma^{\infty}(\mathscr{M}, \mathscr{E}) \mathscr{C}^{\infty}(\mathscr{M})$-linear na primeira variável e tal que, para todo $X \in \Gamma^{\infty}(\mathscr{M}, T \mathscr{M}), \nabla_{X}$ é uma derivação no $\mathscr{C}^{\infty}(\mathscr{M})$ módulo $\Gamma^{\infty}(\mathscr{M}, \mathscr{E})$, i.e.,

$$
\nabla_{X}(f S)=\langle d f, X\rangle S+f \nabla_{X} S, \forall S
$$

Devido a (A.1), a diferença entre duas conexões $\nabla$ e $\tilde{\nabla}$ é uma aplicação $\mathscr{C}^{\infty}(\mathscr{M})$ bilinear a valores em $\Gamma^{\infty}(\mathscr{M}, \mathscr{E})$, i.e., é uma seção de $T \mathscr{M} \otimes \mathscr{E}^{*} \otimes \mathscr{E}$. Uma conexão $\nabla$ induz funtorialmente uma única conexão em todos os fibrados tensoriais $\mathscr{E}^{(r, s)}=$ $\left(\otimes^{r} \mathscr{E}\right) \otimes\left(\otimes^{s} \mathscr{E}^{*}\right)(\mathscr{E} *$ é o fibrado dual a $\mathscr{E})$ por meio da regra de LeIBNiz:

$$
\nabla_{X}\left(S_{1} \otimes S_{2}\right)=\left(\nabla_{X} S_{1}\right) \otimes S_{2}+S_{1} \otimes\left(\nabla_{X} S_{2}\right),\left(\nabla_{X} S^{\prime}\right)(S)+S^{\prime}\left(\nabla_{X} S\right)=\left\langle d S^{\prime}(S), X\right\rangle .
$$

No caso $\mathscr{E}=T \mathscr{M}$, dizemos que $\nabla$ é simétrica se o tensor de torção $T(X, Y) \doteq$ $\nabla_{X} Y-\nabla_{Y} X-[X, Y]$ se anula para todo $X, Y\left([X, Y]=£_{X} Y\right.$ denota o colchete 
de LiE de $X$ com $Y$ ). Numa variedade semi-Riemanniana $(\mathscr{M}, g)$, uma conexão de Levi-Civita é a (única!) conexão simétrica $\nabla$ em $T \mathscr{M}$ que satisfaz $\nabla_{X} g=0$ para todo $X$. Usamos, para esta, a notação $\nabla_{a}$ para denotar seu caráter tensorial.

O tensor de curvatura de RIEMANn $\operatorname{Riem}(g)$ associado a $g$ (mais precisamente, associado a $\nabla_{a}$ ) é dado por $2 \nabla_{[a} \nabla_{b]} X_{c}=\operatorname{Riem}(g)_{a b c}^{d} X_{d}$, para todo $X_{a}$. Denotamos ainda o tensor de RicCi e a curvatura escalar associados a $g$ respectivamente por $\operatorname{Ric}(g)_{a b} \doteq \operatorname{Riem}(g)_{a c b}^{c}$ e $R(g)=g^{a b} \operatorname{Ric}(g)_{a b}$, onde $g^{a b} \doteq\left(g^{-1}\right)^{a b}, g^{a b} g_{b c}=\delta_{c}^{a}$. O tensor de Weyl $C(g)$ associado a $g$ é dado, em $d \geq 3$, por

$$
C_{a b c d}=\operatorname{Riem}_{a b c d}-\frac{2}{d-2}\left(g_{a[c} \operatorname{Ric}_{d] b}-g_{b[c} \operatorname{Ric}_{a] d}\right)+\frac{2}{(d-1)(d-2)} R_{a[c} g_{d] b} .
$$

Esta expressão se anula algebricamente para $d \leq 3$, e, neste caso, Riem $(g)$ é completamente determinado por $\operatorname{Ric}(g)$, através da fórmula (A.2). Adotamos nesta fórmula, assim como em vários lugares ao longo do presente trabalho, um ligeiro abuso de notação ao omitirmos a especificação de $g$ para o tensor de WEYL. Faremos isso se tal prática não causar confusão, para aliviar a notação).

Dada uma variedade semi-Riemanniana $(\mathscr{M}, g), p \in \mathscr{M}$ dizemos que $X(p) \in T_{p} \mathscr{M}$ é tipo tempo, tipo espaço, causal ou tipo luz (nulo) se, respectivamente, $g(X, X)(p)<0$, $>0, \leq 0$ ou $=0$. Um campo vetorial $X$ é tipo tempo, tipo espaço, causal ou tipo luz / nulo se $X(p)$ o for para todo $p \in \mathscr{M}$. Definimos analogamente covetores e campos de covetores tipo tempo, tipo espaço, causais e tipo luz / nulos trocando $g$ por $g^{-1}$. Tal característica de um (campo de) (co)vetor(es) é dita ser seu caráter causal. O levantamento e abaixamento de índices por $g, g^{-1}$ não modifica o caráter causal.

Uma curva $\mathscr{C}^{\infty}$ por partes $\gamma$ é dita de tipo tempo, espaço ou nula se em cada componente $\mathscr{C}^{\infty} \gamma_{\alpha}, \alpha=1, \ldots, k$, o vetor tangente $\dot{\gamma}^{a}$ satisfaz respectivamente $\dot{\gamma}_{\alpha}^{a} \dot{\gamma}_{\alpha a}<0$, $\dot{\gamma}_{\alpha}^{a} \dot{\gamma}_{\alpha a}>0$ ou $\dot{\gamma}_{\alpha}^{a} \dot{\gamma}_{\alpha a}=0$. $\gamma$ é dita causal se $\dot{\gamma}_{\alpha}^{a} \dot{\gamma}_{\alpha a} \leq 0$. Neste caso, $\gamma$ é dita passada ou futura se, para $t^{a}$ denotando um campo vetorial de tipo tempo que determina a orientação temporal de $\left(\mathscr{V}, g_{a b}\right)$, temos respectivamente $t^{a} \dot{\gamma}_{\alpha a}>0$ ou $t^{a} \dot{\gamma}_{\alpha a}<0$ ao longo de cada componente $\gamma_{\alpha}$.

Uma curva $\mathscr{C}^{\infty} \gamma$ é uma geodésica se seu vetor tangente é covariantemente constante: $\dot{\gamma}^{a} \nabla_{a} \dot{\gamma}_{b}=0$. Assim, uma geodésica é unicamente determinada pela escolha de um ponto $p$ de $\mathscr{M}$ e de um vetor tangente a $p$, pelo teorema de existência e unicidade de equações diferenciais ordinárias em variedades. Dados $\mathscr{O}_{1} \subset \mathscr{O} \subset \mathscr{M}$, dizemos que $\mathscr{O}_{1}$ é geodesicamente convexo em relação a $\mathscr{O}$ se, dados quaisquer $p, q \in \mathscr{O}_{1}$, existe uma único segmento geodésico contido em $\mathscr{O}$ ligando $p$ e $q$. Todo $p \in \mathscr{M}$ possui uma vizinhança geodesicamente convexa. Mais em geral, uma vizinhança $\mathscr{U}$ de $p \in \mathscr{M}$ é dita geodesicamente normal se para todo $q \in \mathscr{U}$ existe uma única geodésica ligando 
$p$ a $q$ (i.e., não assumimos que isto é verdade para $q, q^{\prime} \neq p$ ). Para tais vizinhanças, podemos escrever o seguinte sistema de coordenadas: a aplicação exponencial

$$
\begin{aligned}
& \exp _{p}: \mathscr{V} \subset T_{p} \mathscr{M} \rightarrow \mathscr{U} \\
& X \mapsto \exp _{p}(X)=\gamma_{X}(1),
\end{aligned}
$$

onde $\gamma_{X}:[0,1] \rightarrow \mathscr{M}$ é o (único) segmento geodésico tal que $\gamma(0)=p$ e $\dot{\gamma}(0)=X$, é um difeomorfismo para $\mathscr{U}$ suficientemente pequena. Neste caso, $\exp _{p}^{-1}(\mathscr{U})=\mathscr{V}$ é uma vizinhança estrelada ("star-shaped") da origem em $T_{p} \mathscr{M} \cong \mathbb{R}^{d}$ (i.e., dado $q \in \mathscr{V}$, temos que $t q \in \mathscr{V}$ para todo $t \in[0,1])$, cujas coordenadas Cartesianas $\left(\exp _{p}^{-1}\right)^{\mu} \doteq x^{\mu}: \mathscr{U} \rightarrow$ $\mathscr{V}$ são denominadas coordenadas normais. A métrica em $\mathscr{U}$, expressa por meio destas coordenadas, fica [BGM71, Spi79]

$$
\begin{aligned}
g_{\mu \nu}(p) & =\left(g \circ x^{-1}\right)_{\mu \nu}(0)=\eta_{\mu \nu}, \partial_{\mu}\left(g \circ x^{-1}\right)(0)=0 ; \\
g_{\mu \nu}\left(\exp _{p}(x)\right) & =\eta_{\mu \nu}-\frac{1}{3} \operatorname{Riem}\left(g \circ x^{-1}\right)(0)_{\mu \rho \nu \sigma} x^{\rho} x^{\sigma}+o\left(x^{2}\right) ; \\
& \Downarrow \\
\sqrt{\left|\operatorname{det}\left(g \circ x^{-1}\right)\right|}\left(\exp _{p}(x)\right) & =1-\frac{1}{6} \operatorname{Ric}\left(g \circ x^{-1}\right)(0)_{\rho \sigma} x^{\rho} x^{\sigma}+o\left(x^{2}\right) .
\end{aligned}
$$

As fórmulas (A.4)-(A.5), já presentes em forma embrionária na célebre Habilitationsschrift de B. RIEMANN, foram demonstradas em completa generalidade por ÉLIE Cartan.

No caso de variedades Lorentzianas, dizemos que uma hipersuperfície (i.e., uma subvariedade de codimensão 1) é tipo tempo, tipo espaço ou tipo luz / nula se a normal $n^{a}$ em cada ponto for respectivamente um vetor tipo espaço, tipo tempo ou tipo luz. Hipersuperfícies tipo tempo e tipo espaço, quando dotadas do "pullback" de $g$ pela aplicação de inclusão (denominada por vezes primeira forma fundamental), são respectivamente variedades Riemannianas e Lorentzianas. O caso nulo é excepcional, pois nesse caso a normal é também tangente à hipersuperfície, e o "pullback" de $g$ resulta numa forma bilinear simétrica degenerada, pois o subespaço unidimensional de vetores tangentes nulos possui autovalor 0 - as direções restantes são necessariamente tipo espaço, pois não existem dois vetores linearmente independentes e mutuamente ortogonais com respeito a $g$ tais que um é tipo luz e o outro causal.

Seja uma hipersuperfície não-degenerada $\Sigma$ numa variedade Lorentziana $(\mathscr{M}, g)$ (i.e., $\Sigma$ tipo tempo ou espaço), com normal $n^{a}$ e métrica induzida $h_{a b}=g_{a b}-g(n, n) n_{a} n_{b}$ (tal que $h(X, X)=g(X, X)$ se $X \in T \Sigma$, e $h(n,)=$.0 ). Podemos definir, para um $\epsilon>0$, coordenadas geodésicas normais $\left(p, \epsilon^{\prime}\right)$ numa vizinhança tubular $\mathscr{U}$ de $\Sigma$ em $\mathscr{M}$ definindo $\Sigma \times\{0\} \doteq \Sigma$ e, para $p \in \Sigma$ e $\epsilon^{\prime}$ satisfazendo $\left|\epsilon^{\prime}\right|<\epsilon$ ( $\epsilon$ pode depender de $p$, 
a menos que $\Sigma$ seja compacta), defina $\left(p, \epsilon^{\prime}\right)=\gamma\left(\epsilon^{\prime}\right)$, onde $\gamma$ é a única geodésica com $\gamma(0)=p$ e $\dot{\gamma}(0)^{a}=n^{a}(p)$ - com isto, podemos estender $n^{a}$ de $\Sigma$ a $\mathscr{U}$, onde permanece valendo que $g(n, n)= \pm 1$ (usamos a mesma notação para a extensão de $n$, uma vez que não há confusão). Como, neste caso, vemos que $g(n,)=.d \phi$, onde $\phi: \mathscr{U} \rightarrow \mathbb{R}$ é dada por $\phi\left(\left(p, \epsilon^{\prime}\right)=\epsilon^{\prime}\right.$, segue que $\nabla_{a} n_{b}=\nabla_{b} n_{a}$ e $n^{a} \nabla_{a} n_{b}=n^{a} \nabla_{b} n_{a}=0$.

Usaremos do seguinte expediente para relacionar as geometrias intrínseca (dada por $h$ ) e extrínseca (dada por $g$ ) de $\Sigma$ : seja $\nabla^{(0)}$ a conexão de LEvi-CivitA associada à restrição $g^{(0)}$ de $g$ a $\Sigma$ (= "pullback" de $g$ pela aplicação / mergulho natural $i^{\Sigma, \mathscr{M}}$ de inclusão de $\Sigma$ em $\mathscr{M}$ ), e identifiquemos $X, Y$ campos vetoriais em $\Sigma$ com os campos vetoriais em $\mathscr{U}$ obtidos por meio de transporte paralelo de $X$ e $Y$ ao longo das geodésicas normais a $\Sigma$. Podemos, então, identificar a conexão $\nabla^{(0)}$ com a componente de $\nabla_{X} Y$ tangencial a $\Sigma$, i.e.,

$$
\nabla_{X}^{(0)} Y=\left(g^{-1} h\right) \nabla_{X} Y
$$

onde $\left(g^{-1} h\right)_{b}^{a}=g^{a c} h_{b c}=h_{b}^{a}$ é o projetor no subespaço tangente à folheação. A componente de $\nabla_{X} Y$ normal a $\Sigma$, dada por $g\left(\nabla_{X} Y, n\right)=-X^{a} Y^{b} \nabla_{a} n_{b}$, define a curvatura extrínseca (ou segunda forma fundamental) $K_{a b} \doteq-\frac{1}{2}\left(£_{n} h\right)_{a b}=-\nabla_{a} n_{b}$. Notar que, devido às considerações acima, $K$ é simétrico e realmente um tensor $\mathrm{em} \Sigma$. A relação desejada é, então, dada pelas equações de Gauss (A.7) e de CodAzzi-Mainardi (A.8)

$$
\begin{aligned}
h_{a}^{q} h_{b}^{r} h_{c}^{s} h_{d}^{t} \operatorname{Riem}(g)_{q r s t} & =\operatorname{Riem}(h)_{a b c d}-2 g(n, n) K_{a[c} K_{d] b}, \\
h_{b}^{c} n^{d} \operatorname{Ric}(g)_{c d} & =2 h_{[a}^{c} D_{|c|} K_{b]}^{a} .
\end{aligned}
$$

O caso tipo luz pode ser tratado da seguinte maneira: tomando-se uma hipersuperfície tipo luz $\mathscr{S}$, vemos que as geodésicas tipo luz construídas como no caso não-degenerado acima são, ao mesmo tempo, normais e tangentes a $\mathscr{S}$, e geram esta última, no sentido de que é possível escolher uma parametrização afim $\lambda$ comum a todas estas geodésicas (usando, por exemplo, uma métrica Riemanniana auxiliar em $\mathscr{M}$ para normalizar as direções tipo luz), de modo que as subvariedades de $\lambda$ constante são subvariedades espaciais de $\mathscr{M}$ de codimensão 2, com normal nula e tangente a $\mathscr{S}$ denotada por $k^{a}=\left(\frac{d}{d \lambda}\right)^{a}$. É possível, nesta situação, impor a seguinte estrutura Riemanniana em $\mathscr{S}$ : em cada $p \in \mathscr{S}$, cujo parâmetro afim assume o valor $\lambda_{p}$, dizemos que $X(p), Y(p) \in T_{p} \mathscr{S}$ são equivalentes se $X(p)-Y(p)$ é tipo luz, denotando tal classe de equivalência por $\hat{X}(p)(=\hat{Y}(p))$. Ou seja, tomamos em cada $p$ o quociente $\widehat{T_{p} \mathscr{S}} \ni \hat{X}(p)$ de $T_{p} \mathscr{S}$ pelo seu subespaço unidimensional degenerado. A métrica $\hat{h}$ induzida em $T \mathscr{S}$ pelo quociente é uma métrica Riemanniana não-degenerada com uma dimensão a menos, que é identificada em cada subvariedade de $\lambda$ constante com a métrica induzida diretamente por $g$. Assim, podemos definir a curvatura extrínseca nula $\hat{K}=-\frac{1}{2} \frac{d}{d \lambda} \hat{h}$.

Podemos visualizar localmente uma hipersuperfície $\Sigma$ não-degenerada como uma parametrização da família de geodésicas normais a $\Sigma$. Podemos, mais geralmente, con- 
siderar uma família (ou congruência) de geodésicas de mesmo caráter causal, passando por um aberto $\mathscr{O} \subset \mathscr{M}$, tal que, por cada $p \in \mathscr{O}$, passa uma única geodésica da família (localmente, qualquer campo vetorial $X^{a}$ gera uma família de curvas com esta propriedade, que são geodésicas se $\left.X^{a} \nabla_{a} X^{b}=0\right)$. A diferença em relação ao caso de hipersuperfícies é que a distribuição de subespaços de $T_{p} \mathscr{O}$ normais à congruência em cada $p$ não precisa ser integrável, isto é, não precisa gerar uma família de hipersuperfícies normais à congruência. Podemos, não obstante, ainda neste caso definir a primeira forma fundamental da congruência no caso não-degenerado $h_{a b} \doteq g_{a b}-g(X, X) X_{a} X_{b}$ e no caso tipo luz $\hat{h}_{a b} \doteq\left(g_{a b}-X_{a} X_{b}\right) /^{\wedge}$, e a segunda forma fundamental (nula) $\stackrel{(\wedge)}{K}_{a b}=\nabla_{b} X_{a}(/ \wedge),{ }^{1}$ onde $X$ é o campo (co)vetorial tangente à congruência, a qual é parametrizada de maneira tal que $X$ é normalizado em \pm 1 no caso não-degenerado (resp. normalizado em 1 em relação a uma métrica Riemanniana auxiliar no caso tipo luz).

É imediato ver que, no caso não-degenerado, $K$ age nos subespaços normais à congruência, pois $X^{a} K_{a b}=\frac{1}{2} \nabla_{b} X^{a} X_{a}=0$ e $X^{b} K_{a b}=X^{b} \nabla_{b} X_{a}=0$. No caso nulo, $K$ (sem o chapéu) age não-trivialmente no subespaço normal tipo espaço $e$ na outra direção nula normal a este subespaço, cujo campo vetorial tangente de mesma orientação temporal que $X^{a}$ denotamos por $\bar{X}^{a}$. Este campo vetorial gera localmente uma outra congruência de geodésicas nulas, naturalmente associada à primeira - a saber, é a única outra congruência que compartilha os mesmos subespaços normais tipo espaço. A normalização adotada para $\bar{X}^{a}$ é dada por $g(X, \bar{X})=-2$.

Ao contrário do caso de hipersuperfícies, no entanto, $K_{a b}$ não é necessariamente um tensor simétrico: $\stackrel{(\wedge)}{\omega}_{a b} \doteq \stackrel{(\wedge)}{K}_{[a b]}$ (denominado torção ("twist") (nula) da congruência) expressa precisamente a falha da distribuição de subespaços normais à congruência em ser integrável. Pelo teorema de Frobenius [Wal84], a congruência é normal a uma família de hipersuperfícies se e somente se $\stackrel{(\Lambda)}{K}_{[a b]} \equiv 0$. A parte simétrica pode ser decomposta em termos do traço em relação a $\stackrel{(\wedge)}{h}$, denominado expansão (ou curvatura média) (nula) $\theta \doteq \stackrel{(\wedge)}{K}_{a b} \stackrel{(\wedge)}{h}^{a b}$ (o chapéu no caso nulo é desnecessário no primeiro membro por se tratar de um escalar), e da parte de traço zero, denominada cisalhamento ("shear") (nulo) $\stackrel{(\wedge)}{\sigma}_{a b} \doteq \stackrel{(\wedge)}{K}_{(a b)}-\frac{1}{d-1} \stackrel{(\wedge)}{\theta}^{\prime} \cdot \stackrel{(n)}{h}_{a b}$ (o fator $(d-1)$ deve ser substituído por $(d-2)$ no caso nulo, por causa do abaixamento de dimensão devido ao quociente pela relação de equivalência associada $\mathrm{a}^{\wedge}$ ). Em suma,

$$
\stackrel{(\wedge)}{K}_{a b}=\stackrel{(\wedge)}{\omega}_{a b}+\stackrel{(\wedge)}{\sigma}_{a b}+\frac{1}{d-1(2)} \stackrel{(\wedge)}{h}_{a b}
$$

\footnotetext{
${ }^{1}$ Usamos, para congruências, uma convenção de sinal oposta à empregada para hipersuperfícies ao definirmos a segunda forma fundamental.
} 
A maneira como a geometria normal à congruência muda ao longo do transporte paralelo é dada pela equação matricial de RiCCATi

$$
X^{c} \nabla_{c} K_{a b}=-K_{b}^{c} K_{a c}+\operatorname{Riem}(g)_{c b a d} X^{c} X^{d} .
$$

Vamos tomar o quociente de (A.10) no caso nulo, que é o caso de interesse no presente trabalho, e decompor o resultado em termos da expansão, cisalhamento e torção no primeiro membro. Denotando por $\lambda$ a parametrização afim comum da congruência, temos:

$$
\begin{aligned}
\frac{d \theta}{d \lambda} & =-\frac{1}{d-2} \theta^{2}-\hat{\sigma}^{a b} \hat{\sigma}_{a b}+\hat{\omega}^{a b} \hat{\omega}_{a b}-R_{c d} X^{c} X^{d} ; \\
X^{c} \nabla_{c} \hat{\sigma}_{a b} & =-\theta \hat{\sigma}_{a b}+C(g)_{c b a d} X^{c} X^{d} ;{ }^{2} \\
X^{c} \nabla_{c} \hat{\omega}_{a b} & =-\theta \hat{\omega}_{a b} .
\end{aligned}
$$

A equação (A.11) é a celebrada equação de RAYCHAUDHURI.

Qualquer subfamília uniparamétrica de geodésicas $\gamma_{\lambda},-\epsilon<\lambda<\epsilon, \epsilon>0$ de uma congruência produz um campo vetorial $\left.Y \doteq \frac{d}{d \lambda}\right|_{\lambda=0}$ em $\gamma_{0}$, que denota o deslocamento relativo de uma geodésica "infinitesimalmente próxima a $\gamma_{0}$ ". A aceleração relativa de duas tais geodésicas é dada pela equação de desvio geodésico (também denominada equação de JACOBI)

$$
X^{c} \nabla_{c}\left(X^{b} \nabla_{b} Y^{a}\right)=-R_{c b d}^{a} X^{c} X^{d} Y^{b} .
$$

Mais em geral, podemos definir um campo de JACOBI numa geodésica $\gamma$ como uma solução $Y$ de (A.14) com $X=\dot{\gamma}$. Dizemos que uma geodésica causal $\gamma$ possui um par de pontos conjugados $p=\gamma\left(\lambda_{1}\right), q=\gamma\left(\lambda_{2}\right), \lambda_{1}<\lambda_{2}$, se existe um campo de JACOBI $Y$ que não se anula em $\gamma\left(\left(\lambda_{1}, \lambda_{2}\right)\right)$ e se anula em $p$ e $q$. Geometricamente, isto significa que as geodésicas emanando de $p$ e "infinitesimalmente próximas" a $\gamma$ tendem a se focalizar em $q$.

Para visualizar esse fenômeno no caso não-degenerado, tomemos um referencial ortonormal $e_{i}^{a}(\lambda), i=0, \ldots, d-1$ paralelamente propagado ao longo de $\gamma$, tal que $e_{0}^{a}(\lambda)=X^{a}(\lambda)$. Tomando $\lambda_{1}=0$ por simplicidade, temos $Y^{a}(0)=0$ e, portanto,

$$
Y^{i}(\lambda)=\sum_{j=1}^{d-1} A_{j}^{i}(\lambda) \frac{d Y^{j}}{d \lambda}(0),
$$

\footnotetext{
${ }^{2} C(g)_{c b a d} X^{c} X^{d}$ é um tensor simétrico que assume um único valor em cada classe de equivalência associada $\mathrm{a}^{\wedge}$.
} 
onde a matriz $A_{j}^{i}$ expressa em termos do referencial escolhido satisfaz

$$
\frac{d^{2} A_{j}^{i}}{d \lambda^{2}}=-R_{k l m}^{i} X^{k} X^{m} A_{j}^{l}
$$

Como

$$
\frac{d Y^{i}}{d \lambda}=e_{b}^{i} X^{a} \nabla_{a} Y^{b}=e_{b}^{i} Y^{a} \nabla_{a} X^{b}=\sum_{j=1}^{d-1} K_{j}^{i} Y^{j}
$$

temos que

$$
K_{j}^{i}=\left[\frac{d A}{d \lambda}\right]_{k}^{i}\left[A^{-1}\right]_{j}^{k},
$$

e, daí, $\theta=\operatorname{tr}[K]=\frac{1}{\operatorname{det} A} \frac{d}{d \lambda}(\operatorname{det} A)$. Logo, $\operatorname{det} A \rightarrow 0$, o que implica que $A$ possui um subespaço de autovetores normais a $X^{a}$ com autovalor 0 e, portanto, existe um campo de JACOBI que se anula em $p$ e $q$, se e somente se $\theta \rightarrow-\infty$ em $q$. O caso tipo luz é tratado adotando-se um referencial paralelamente propagado ao longo de $\gamma$ tal que $e_{0}^{a}=X^{a}$, $e_{1}^{a}=\bar{X}^{a}$, e $e_{2}^{a}, \ldots, e_{d-1}^{a}$ um conjunto ortonormal tal que $g\left(e_{0}, e_{i}\right)=g\left(e_{1}, e_{i}\right)=0$, $i=2, \ldots, d-1$. As fórmulas acima não envolvem $e_{1}^{a}$ neste caso.

\section{A.2 Teoria global}

Por espaço-tempo entende-se uma variedade Lorentziana $(\mathscr{M}, g)$ temporalmente orientável, i.e., existe um campo vetorial $\mathscr{C}^{\infty} T^{a}$ tipo tempo em $\mathscr{M}$ que não se anula em parte alguma (em particular, podemos escolher $g(T, T)=-1$ ).

\section{A.2.1 Estrutura causal, completeza geodésica}

Seja $\left(\mathscr{M}, g_{a b}\right)$ um espaço-tempo, $\mathscr{O} \subset \mathscr{M}, p \in \mathscr{O}$. O futuro cronológico (resp. causal) de $p$ em relação a $\mathscr{O}$, denotado por $I^{+}(p, \mathscr{O})$ (resp. $\left.I^{+}(p, \mathscr{O})\right)$ é dado pelos seguintes conjuntos:

$$
\begin{aligned}
I^{+}(p, \mathscr{O})= & \left\{x \in \mathscr{O}: \exists \gamma:[0, a] \stackrel{\mathscr{C}^{\infty}}{\longrightarrow} \mathscr{O}\right. \text { de tipo tempo } \\
& \text { e futura tal que } \gamma(0)=p, \gamma(a)=x\} \\
J^{+}(p, \mathscr{O})= & \left\{x \in \mathscr{O}: x=p \text { ou } \exists \gamma:[0, a] \stackrel{\mathscr{C}^{\infty}}{\longrightarrow} \mathscr{U}\right. \text { causal } \\
& \text { e futura tal que } \gamma(0)=p, \gamma(a)=x\} .
\end{aligned}
$$

Trocando-se futuro por passado, define-se de maneira dual o passado cronológico (resp. causal) $I^{-}(p, \mathscr{O})\left(\right.$ resp. $\left.J^{-}(p, \mathscr{O})\right)$ de $p$ em relação a $\mathscr{O}$. Seguem dessas definições 
que $I^{ \pm}(p, \mathscr{O})$ é aberto e $\operatorname{int}\left(J^{ \pm}(p, \mathscr{O})\right)=I^{ \pm}(p, \mathscr{O})$. Um conjunto $\mathscr{O}_{1} \subset \mathscr{O}$ é dito ser causalmente convexo em relação a $\mathscr{O}$ se, dados quaisquer $p \leq_{\mathscr{O}} q \in \mathscr{O}_{1}$, temos que $J^{+}(p, \mathscr{O}) \cap J^{-}(q, \mathscr{O}) \subset \mathscr{O}_{1}$.

Usando-se esses conjuntos acima podemos definir relações de cronologia e causalidade entre dois pontos. Sejam $p, q \in \mathscr{O} \subset \mathscr{M}$. Dizemos que $p$ precede cronologicamente (resp. causalmente) $q$ em relação a $\mathscr{O}$ se $p \in I^{-}(q, \mathscr{O})$ (resp. $p \in J^{-}(q, \mathscr{O})$ ). Denotamos essa relação por $p \ll_{\mathscr{O}} q$ (resp. $p \leq_{\mathscr{O}} q$ ). Equivalentemente, dizemos neste caso que $q$ sucede cronologicamente (resp. causalmente) $p$ em relação a $\mathscr{O}$, com a notação $q \gg_{\mathscr{O}} p$ (resp. $q \geq_{\mathscr{O}} p$ ). Se $p \leq_{\mathscr{O}} q$ e $p \neq q$, escrevemos $p<_{\mathscr{O}} q$, ou, dualmente, $q>_{\mathscr{O}} p$. Se $p \neq q$, $p K_{\mathscr{O}} q$ e $p \gg_{\mathscr{O}} q$ (resp. $p \nless_{\mathfrak{O}} q$ e $p \ngtr_{\mathscr{O}} q$ ), dizemos que $p$ e $q$ são cronologicamente (resp. causalmente) disjuntos - neste caso, escrevemos $p \curlywedge_{\mathscr{O}} q$ (resp. $p \perp_{\mathscr{O}} q$ ). Todas as relações de cronologia e causalidade definidas acima, bem como o futuro e passado cronológicos ou causais, são definidos para conjuntos não-unitários e não-vazios de maneira óbvia. Se $p \neq q \in \mathscr{O} \subset \mathscr{M}$ implica $p \curlywedge_{\mathscr{O}} q$ (resp. $\left.p \perp_{\mathscr{O}} q\right)$, dizemos que $\mathscr{O}$ é acronal (resp. acausal) em relação a $\mathscr{O}$.

Algumas consequências das definições do parágrafo anterior são:

- $\ll_{\mathscr{O}}$ é uma relação aberta, ou seja,

$$
\begin{aligned}
p \ll_{\mathscr{O}} q & \Rightarrow \exists \mathscr{O}_{1}, \mathscr{O}_{2} \subset \mathscr{M} \text { abertos tais que } \\
p & \in \mathscr{O}_{1}, q \in \mathscr{O}_{2} \text { e } \mathscr{O}_{1} \cap \mathscr{O} \ll \mathscr{O} \mathscr{O}_{2} \cap \mathscr{O} .
\end{aligned}
$$

$$
J^{+}\left(J^{+}(p, \mathscr{O}), \mathscr{O}\right)=J^{+}(p, \mathscr{O}) \text {, i.e., } p \leq_{\mathscr{O}} q \text { e } q \leq_{\mathscr{O}} r \Rightarrow p \leq_{\mathscr{O}} r .
$$

$$
\begin{array}{r}
I^{+}\left(I^{+}(p, \mathscr{O}), \mathscr{O}\right)=I^{+}\left(J^{+}(p, \mathscr{O}), \mathscr{O}\right)=J^{+}\left(I^{+}(p, \mathscr{O}), \mathscr{O}\right)=I^{+}(p, \mathscr{O}), \\
\text { i.e., }\left(p \ll_{\mathscr{O}} q \text { e } q \ll_{\mathscr{O}} r\right) \text { ou }\left(p \ll_{\mathscr{O}} q \text { e } q \leq_{\mathscr{O}} r\right) \text { ou }\left(p \leq_{\mathscr{O}} q\right. \text { e } \\
\left.q \ll_{\mathscr{O}} r\right) \Rightarrow p \ll_{\mathscr{O}} r .
\end{array}
$$

$$
\overline{I^{+}\left(\mathscr{O}_{1}, \mathscr{O}_{2}\right)}=\overline{J^{+}\left(\mathscr{O}_{1}, \mathscr{O}_{2}\right)}, \forall \mathscr{O}_{1} \subset \mathscr{O}_{2}
$$

onde o fecho é tomado na topologia relativa de $\mathscr{O}_{2}$.

$$
\partial I^{+}\left(\mathscr{O}_{1}, \mathscr{O}_{2}\right)=\partial J^{+}\left(\mathscr{O}_{1}, \mathscr{O}_{2}\right), \forall \mathscr{O}_{1} \subset \mathscr{O}_{2},
$$

onde a fronteira é tomada na topologia relativa de $\mathscr{O}_{2}$. 
O conjunto definido em (A.21) é denominado fronteira acronal futura de $\mathscr{O}_{1} \mathrm{em}$ relação a $\mathscr{O}_{2}$, e constitui uma subvariedade topológica e acronal de $\mathscr{M}$ tal que todo $p \in \partial I^{+}\left(\mathscr{O}_{1}, \mathscr{O}_{2}\right)$ pertence a um (necessariamente único) segmento geodésico, acronal em relação a $\mathscr{O}_{2}$ e contido em $\partial I^{+}\left(\mathscr{O}_{1}, \mathscr{O}_{2}\right)$, que ou é inextensível no passado ou possui um extremo passado em $\mathscr{O}_{1}$. Tais geodésicas são denominadas geradores de $\partial I^{+}\left(\mathscr{O}_{1}, \mathscr{O}_{2}\right)$. Note que (A.17)-(A.21) continuam valendo se trocarmos o futuro pelo passado.

Uma das ferramentas mais poderosas na análise da geometria global de variedades Lorentzianas é a distância Lorentziana. Sejam $p \leq \mathscr{M} q \in \mathscr{M}$, e denote por $\Omega_{p, q} \mathrm{O}$ espaço de curvas causais futuras $\mathscr{C}^{\infty}$ por partes ligando $p$ a $q$ (este espaço é vazio se $p \not \leq \mathscr{M} q) . \Omega_{p, q}$ herda a seguinte topologia do espaço $\operatorname{Path}(\mathscr{M})$ de curvas $\mathscr{C}^{0}$ em $\mathscr{M}$ : dizemos que uma seqüência de curvas $\mathscr{C}^{\infty}$ por partes $\left\{\gamma_{n}:[0, \lambda] \rightarrow \mathscr{M}\right\}$ converge para $\gamma:[0, \lambda] \rightarrow \mathscr{M}$ se $\gamma_{n}(0) \rightarrow \gamma(0)$ e, dado qualquer aberto $\mathscr{O} \subset \mathscr{M}$ contendo $\gamma([0, \lambda])$, existe $N \in \mathbb{Z}_{+}$tal que $\gamma_{n}([0, \lambda]) \subset \mathscr{O}$ para todo $n \geq N$. Esta topologia é denominada topologia $\mathscr{C}^{0}$. Considere $\lambda \in \Omega_{p, q}$ parametrizada por $\lambda \in\left[\lambda_{0}=0, \lambda_{k}\right]$, com componentes $\mathscr{C}^{\infty} \gamma_{i}, i=1, \ldots, k$, definidas respectivamente nos intervalos $\left[\lambda_{i-1}, \lambda_{i}\right]$, $\lambda_{0}<\lambda_{1}<\cdots<\lambda_{k}$. O comprimento de arco Lorentziano de $\gamma$ é dado por

$$
L_{g}(\gamma) \doteq \sum_{i=1}^{k} \int_{\lambda_{i-1}}^{\lambda_{i}} \sqrt{-g\left(\dot{\gamma}_{i}(\lambda), \dot{\gamma}_{i}(\lambda)\right)} d \lambda .
$$

Para curvas de tipo tempo, que correspondem a trajetórias de observadores, (A.22) também é dita ser o tempo próprio da trajetória.

Definição A.1 A distância Lorentziana em $(\mathscr{M}, g)$ é a função $d_{g}: \mathscr{M} \times \mathscr{M} \rightarrow \overline{\mathbb{R}}_{+} \cup$ $\{+\infty\}$ dada por

$$
(p, q) \mapsto d_{g}(p, q)=\left\{\begin{aligned}
\sup _{\gamma \in \Omega, q} L_{g}(\gamma) & \text { se } p \leq \mathscr{M} q \\
0 & \text { de outra forma }
\end{aligned}\right.
$$

Segue imediatamente da definição que $d_{g}$ satisfaz uma desigualdade triangular reversa: se $p \leq \mathscr{M} r \leq \mathscr{M} q$, então

$$
d_{g}(p, q) \geq d_{g}(p, r)+d_{g}(r, q) .
$$

Um exemplo onde o valor $d_{g}(p, q)=+\infty$ pode ser obtido é no espaço-tempo de Reissner-Nordström extremal (ver [BEE96]). No caso $p=q$, temos que $d_{g}(p, p)=0$ ou $d_{g}(p, p)=+\infty$. $d_{g}$ é contínua por baixo para todo $(p, q)$ onde $d_{g}(p, q)<+\infty$. Dizemos que $\gamma \in \Omega_{p, q}$ é maximal se $L(\gamma)=d_{g}(p, q)$. Pode-se provar [BEE96] que, se $\gamma$ é maximal, é uma geodésica $\left(\mathscr{C}^{\infty}\right)$ causal a menos de reparametrização. Em particular, uma geodésica nula maximal é acronal. Em contrapartida, se uma geodésica causal, 
digamos, futura $\gamma$ possui um par de pontos conjugados $p \leq q$, então, dados quaisquer pontos $p^{\prime}, q^{\prime}$ em $\gamma$ com $p^{\prime}$ no passado de $p$ e $q^{\prime}$ no futuro de $q$, temos que os segmentos de $\gamma$ que ligam $p^{\prime}$ a $q$ e $p$ a $q^{\prime}$ não mais maximizam o comprimento de arco Lorentziano entre esses pares de pontos - em particular, se $\gamma$ é tipo luz, ela deixa de ser acronal se suficientemente estendida. O mesmo ocorre se uma geodésica causal, distinta de $\gamma$, ligar $p$ e $q$.

Agora, recapitularemos algumas condições de causalidade. Dizemos que o espaçotempo $(\mathscr{M}, g)$ é cronológico (resp. causal) se não existe $p \in \mathscr{M}$ tal que $p \ll \mathscr{M} p$ (resp. $p<\mathscr{M} p)$ - equivalentemente, $(\mathscr{M}, g)$ é cronológico se e somente se $d_{g}(p, p)=0$, e causal se e somente se $\Omega_{p, p}=\varnothing$, para todo $p \in \mathscr{M}$. Dizemos que $(\mathscr{M}, g)$ é fortemente causal se, para todo $p \in \mathscr{M}$, existem vizinhanças abertas arbitrariamente pequenas $\mathscr{O}$ de $p$ tais que nenhuma curva causal possui intersecção desconexa com $\mathscr{O}$. Isso implica que nenhum compacto contém completamente uma curva causal inextensível (i.e., que não está propriamente contida em nenhuma outra curva) no passado ou no futuro, e, em particular, que $(\mathscr{M}, g)$ é causal.

Uma classe particularmente interessante de espaços-tempos é aquela que admite uma folheação por hipersuperfícies acausais, pois estes admitem uma noção global de evolução temporal (possivelmente suplementada por condições de contorno). A saber, dizemos que $t \in \mathscr{C}^{0}(\mathscr{M})$ é uma função tempo global se $t \circ \gamma$ é estritamente crescente para toda curva causal futura $\gamma$. Se $t$ for $\mathscr{C}^{1}$, dizemos equivalentemente que $t$ é uma função tempo global se $d t$ é tipo tempo futuro e $t(\mathscr{M})=\mathbb{R}$. Neste caso, $t^{-1}(\tau)$ é uma hipersuperfície tipo espaço para todo $\tau \in \mathbb{R}$, e todo $p \in \mathscr{M}$ pertence a $t^{-1}(\tau)$ para precisamente um valor de $\tau$. Como $d t$ induz a mesma orientação temporal que a adotada em $(\mathscr{M}, g)$, segue que $t^{-1}(\tau)$ é acausal para todo $\tau \in \mathbb{R}$. Um espaço-tempo que admite uma função tempo global (contínua) é dito estavelmente causal. O nome vem do fato de que tais espaços-tempos podem ser definidos de maneira equivalente pela seguinte propriedade: existe um campo de covetores de tipo tempo $T_{a}$ tal que a métrica Lorentziana $g_{a b}-T_{a} T_{b}$ é cronológica. No nosso caso, $T=d t$; conversamente, dado $T_{a}$, é possível construir uma função tempo global (contínua) [Ger70, Wal84]. A questão de como construir funções tempo globais $\mathscr{C}^{\infty}$ neste contexto foi respondida parcialmente por SEIFERT [Sei77] e DiECKMANn [Die88], e completamente há pouco tempo por BERnAl e SÁNCHEz [BS03, BS05, BS06], eliminando a necessidade de assumir diferenciabilidade de funções tempo globais na definição de causalidade estável. Destarte, como $t$ diferenciável não possui pontos críticos, todas as hipersuperfícies de $t$ constante são difeomorfas entre si.

Por fim, dizemos que um espaço-tempo fortemente causal $(\mathscr{M}, g)$ é causalmente simples se, para todo $p \in \mathscr{M}$ tem-se que $J^{ \pm}(p, \mathscr{M})$ é fechado, ou, equivalentemente, $J^{ \pm}(p, \mathscr{M}) \backslash I^{ \pm}(p, \mathscr{M})=\partial I^{ \pm}(p, \mathscr{M})$, e globalmente hiperbólico se , para todo $p, q \in \mathscr{V}$, 
o conjunto $J^{-}(p, \mathscr{M}) \cap J^{+}(q, \mathscr{M})$ é compacto se não-vazio. ${ }^{3}$ Equivalentemente, $(\mathscr{M}, g)$ é globalmente hiperbólico se e somente se $\Omega_{p, q}$ é compacto na topologia $\mathscr{C}^{0}$ se nãovazio. Daí segue que, num espaço-tempo globalmente hiperbólico, $d_{g}$ é contínua e $d_{g}(p, q)<+\infty, \forall p, q \in \mathscr{M}$. Hiperbolicidade global claramente implica causalidade simples, que, por sua vez, implica causalidade estável (para a última afirmação, ver [BEE96]). Todas as condições acima podem ser definidas para subconjuntos de $\mathscr{M}$.

Seja agora $\mathscr{S} \subset \mathscr{M}$ fechado e acronal. O dominio de dependência futuro (resp. passado) de $\mathscr{S}$, denotado por $D^{+}(\mathscr{S})$ (resp. $D^{-}(\mathscr{S})$ ) consiste no seguinte conjunto:

$D^{+/-}(\mathscr{S})=\{p \in \mathscr{M}: \forall \gamma:[0, a) \longrightarrow \mathscr{M}$ inextensível, causal e passada/futura tal que $\gamma(0)=p, \exists b<a$ tal que $\gamma(b) \in \mathscr{I}\}$.

$D(\mathscr{S}) \doteq D^{+}(\mathscr{S}) \cup D^{-}(\mathscr{S})$ é denotado dominio de dependência ou desenvolvimento de CAUCHY de $\mathscr{S}$. O bordo de $\mathscr{S}$ (notação: $\dot{\mathscr{S}}$ ) consiste dos pontos $p \in \mathscr{S}$ tais que toda vizinhança aberta de $p$ possui pontos $q \in I^{-}(p), r \in I^{+}(p)$ e uma curva $\gamma$ de tipo tempo ligando $q$ a $r$ com intersecção vazia com $\mathscr{S}$. Se $\dot{\mathscr{S}}=\varnothing$, então $\mathscr{S}$ é uma subvariedade topológica de codimensão 1, mergulhada em $\mathscr{M}$. Um domínio de dependência possui as seguintes propriedades:

- O conjunto $\operatorname{int}(D(\mathscr{S}))$ é globalmente hiperbólico (neste caso, dizemos que $S$ é uma superfície de CAUCHY para $\operatorname{int}(D(\mathscr{S}))$ ), assim como também o é $D(\mathscr{S})$ se $\dot{\mathscr{S}}=\varnothing$. Conversamente, pode-se provar que toda região globalmente hiperbólica possui uma superfície de CAUCHY - mais ainda, pode-se construir uma função tempo global $t$ tal que $t^{-1}(\tau)$ é uma superfície de CAUCHY para cada $\tau \in \mathbb{R}$.

- O conjunto fechado e acronal $H^{+}(\mathscr{S}) \doteq \overline{D^{+}(\mathscr{S})} \backslash I^{-}\left(D^{+}(\mathscr{S})\right)$, denominado horizonte de CAUCHY futuro de $\mathscr{S}$ possui a seguinte propriedade: todo $p \in$ $H^{+}(\mathscr{S})$ está contido em um (necessariamente único) segmento geodésico nulo, acronal e contido em $H^{+}(\mathscr{S})$, que ou é inextensível no passado ou possui um extremo passado em $\dot{\mathscr{S}}$. Definição análoga existe para $H^{-}(\mathscr{S})$, o horizonte de CAUChy passado de $\mathscr{S}$;

- O horizonte de Cauchy $H(\mathscr{S}) \doteq H^{+}(\mathscr{S}) \cup H^{-}(\mathscr{S})$ é igual a $\partial D(\mathscr{S})$.

A principal característica de um espaço-tempo globalmente hiperbólico é que ele é "dinamicamente fechado", i.e., o problema de CAUCHY para qualquer equação de movimento que propaga os dados iniciais de maneira localmente causal (i.e., o suporte da solução em cada instante cresce no tempo com velocidade menor que a da luz), como,

\footnotetext{
${ }^{3}$ Muito recentemente, BERnAL e SÁnchEz [BS07] mostraram que podemos substituir "fortemente causal" por "causal" na definição de hiperbolicidade global dada aqui.
} 
por exemplo, a equação de onda, é bem posto, i.e., possui uma única solução, que depende continuamente dos dados iniciais, pois a solução associada a dados iniciais com suporte compacto na hipersuperfície de tempo zero possui suporte espacialmente compacto em cada instante. Isto elimina a necessidade de condições de contorno, o que não ocorre nos casos mais gerais de espaços-tempos estavelmente causais ou causalmente simples [Wal80].

Sobre completeza geodésica: dizemos que uma geodésica futura $\gamma$ é completa no passado, futuro ou simplesmente completa se, respectivamente, seu parâmetro afim pode ser estendido para $\mathbb{R}_{+}, \mathbb{R}_{-}$ou $\mathbb{R}$. $(\mathscr{M}, g)$ é dito espacialmente, temporalmente ou nulamente geodesicamente completo se, respectivamente, toda geodésica de tipo tempo, espaço ou nula for completa. Os contra-exemplos coletados e construídos por GEROCH em [Ger68] mostram que as três definições são logicamente inequivalentes, isto é, nenhuma dessas três definições implica em outra. Incompleteza geodésica temporal ou nula são critérios comumente utilizados para determinar a presença de singularidades.

Concluindo, devemos considerar duas noções importantes para teorias de observáveis locais: o complemento causal de um aberto $\mathscr{O}$ em relação a $\mathscr{O} \supset \mathscr{O}_{1}$ é o conjunto $\left(\mathscr{O}_{1}\right)_{\mathscr{O}}^{\prime} \doteq \operatorname{int}\left\{p \perp_{\mathscr{O}} \mathscr{O}_{1}\right\}$, e o completamento causal de $\mathscr{O}_{1}$ em relação a $\mathscr{O}$ é dado por $\left.\left(\mathscr{O}_{1}\right)_{\mathscr{O}}^{\prime \prime} \equiv\left(\mathscr{O}_{1}\right)_{\mathscr{O}}^{\prime}\right)_{\mathscr{O}}^{\prime} \cdot{ }^{4}$ Se $\left(\mathscr{O}_{1}\right)_{\mathscr{O}}^{\prime \prime}=\mathscr{O}_{1}$, dizemos que $\mathscr{O}_{1}$ é causalmente completo em relação a $\mathscr{O}$. Dessas definições segue que:

- $\left(\mathscr{O}_{1}\right)_{\mathscr{O}}^{\prime \prime}$ é o menor aberto causalmente completo em relação a $\mathscr{O}$ que contém $\mathscr{O}_{1}$. Note aqui a importância de tomarmos $\mathscr{O}_{1}$ aberto na nossa definição de complemento causal - se, por exemplo, $\mathscr{O}_{1}=\{p\}$ ou, mais em geral, um conjunto acronal discreto, temos $\left(\mathscr{O}_{1}\right)_{\mathscr{O}}^{\prime \prime}=\varnothing$. Ver logo adiante como faremos para definir as operações de complemento causal para conjuntos não necessariamente abertos, para os quais a propriedade acima não é obrigatoriamente válida.

- $\left(\mathscr{O}_{1}\right)_{\mathscr{O}}^{\prime \prime \prime}=\left(\mathscr{O}_{1}\right)_{\mathscr{O}}^{\prime}$;

- $\mathscr{O}_{1} \subset \mathscr{O}_{2} \Rightarrow\left(\mathscr{O}_{1}\right)_{\mathscr{O}}^{\prime} \supset\left(\mathscr{O}_{2}\right)_{\mathscr{O}}^{\prime}$;

- $\left(\cup_{\alpha} \mathscr{O}_{\alpha}\right)_{\mathscr{O}}^{\prime}=\cap_{\alpha}\left(\mathscr{O}_{\alpha}\right)_{\mathscr{O}}^{\prime} \Rightarrow\left(\cup_{\alpha}\left(\mathscr{O}_{\alpha}\right)_{\mathscr{O}}^{\prime}\right)_{\mathscr{O}}^{\prime}=\cap_{\alpha}\left(\mathscr{O}_{\alpha}\right)_{\mathscr{O}}^{\prime \prime}$ e, portanto, toda intersecção de conjuntos causalmente completos é causalmente completa - usaremos estas fórmulas para estender a definição de complemento causal a conjuntos arbitrários.

- Todo diamante $\mathscr{O}_{p, q} \doteq I^{-}(p, \mathscr{O}) \cap I^{+}(q, \mathscr{O})=(\{p, q\})_{\mathscr{O}}^{\prime \prime}, p \ll_{\mathscr{O}} q$, é causalmente completo.

${ }^{4}$ Tais definições diferem das adotadas em [Rib07], onde não assumimos $\mathscr{O}$ aberto. As diferenças envolvidas, todavia, não implicam em nenhuma alteração nas demonstrações dos resultados de [Rib07]. 
Ao longo do presente trabalho, assumimos que todos os nossos espaços-tempos são fortemente causais, a menos que seja dito o contrário.

ObservaÇÃo A.2 Se $\mathscr{O}=\mathscr{M}$, a parte das notações desta Subseção indicando $\mathscr{O}$ pode ser omitida.

\section{A.2.2 Infinito conforme}

DEFiniçÃo A.3 O infinito conforme ou fronteira conforme de um espaço-tempo d-dimensional $(\mathscr{M}, g)$ é um espaço-tempo d-1-dimensional $\left(\mathscr{I}, \bar{g}^{(0)}\right)$ tal que existe uma variedade Lorentziana d-dimensional $(\overline{\mathscr{M}}, \bar{g})$ com bordo (o fecho conforme ou completamento conforme de $(\mathscr{M}, g))$ satisfazendo:

- $\mathscr{I} \equiv \partial \overline{\mathscr{M}}$; existe um difeomorfismo $\Phi$ de $\mathscr{M}$ em $\Phi(\mathscr{M}) \doteq \overline{\mathscr{M}} \backslash \partial \overline{\mathscr{M}}$;

- $\bar{g}^{(0)}$ é a métrica semi-Riemanniana (possivelmente degenerada) induzida por $\bar{g}$ em $\mathscr{I} ;$

- Existe um fator conforme ou de WEYL, isto é, uma função z real, positiva e $\mathscr{C}^{\infty}$ em $\mathscr{M}$, que admite uma extensão $\mathscr{C}^{\infty}$ a $\overline{\mathscr{M}}$ tal que $z\lceil\mathscr{I} \equiv 0$ e dz「ף $\neq 0$ em $\mathscr{I}$ (o fator de WEYL e sua extensão ao fecho conforme são sempre denotados pela mesma letra, uma vez que não há confusão aqui) satisfazendo $\bar{g}=z^{2} g$. Denotamos respectivamente por $\bar{\nabla}_{a} e^{(0)} \bar{\nabla}_{a}$ as conexões de LEVI-CIVITA associadas a $\bar{g}$ e $\bar{g}^{(0)}$.

Mostraremos agora como ficam as fórmulas para $\bar{\nabla}_{a}$, $\operatorname{Riem}(\bar{g})$ e $\operatorname{Ric}(\bar{g})$ em $\mathscr{M}$. Primeiramente, lembremos que quaisquer duas conexões lineares $\nabla_{a}$ e $\bar{\nabla}_{a}$ diferem em sua ação sobre o $\mathscr{C}^{\infty}$-módulo $\Gamma\left(\mathscr{M}, T^{*} \mathscr{M}\right)$ por um tensor $C_{a b}^{c}$ de posto $(1,2)$, i.e.,

$$
\bar{\nabla}_{a} X_{b}=\nabla_{a} X_{b}-C_{a b}^{c} X_{c}
$$

Assim, a partir das condições de LEvi-Civita

$$
\nabla_{a} g_{b c}=0, \nabla_{a} \nabla_{b} f=\nabla_{b} \nabla_{a} f, \forall f \in \mathscr{C}^{\infty}(\mathscr{M}),
$$

obtemos

$$
C_{a b}^{c}=\frac{1}{2} \bar{g}^{c d}\left(\nabla_{a} \bar{g}_{b d}+\nabla_{b} \bar{g}_{a d}-\nabla_{d} \bar{g}_{a b}\right) .^{5}
$$

Em particular, tomando $g$ e $\bar{g}$ como na Definição A.3, segue que $\nabla_{a} \bar{g}_{b c}=2 z g_{b c} \nabla_{a} z$ e, portanto,

$$
C_{a b}^{c}=\frac{1}{z} g^{c d}\left(g_{b d} \nabla_{a} z+g_{a d} \nabla_{b} z-g_{a b} \nabla_{d} z\right)
$$

\footnotetext{
${ }^{5}$ Especializando esta fórmula no caso de uma carta local e tomando a métrica de MiNkOWsKI, obtemos assim a expressão usual em coordenadas locais para os símbolos de CHRISTOfFel.
} 
Geodésicas em relação a $\nabla_{a}$ em geral não mais o são em relação a $\bar{\nabla}_{a}$, pois

$$
X^{a} \nabla_{a} X^{b}=0 \Rightarrow X^{a} \bar{\nabla}_{a} X^{b}=X^{a} C_{a c}^{b} X^{c}=\frac{2}{z} X^{b} X^{c} \nabla_{c} z-\frac{g(X, X)}{z} g^{b d} \nabla_{d} z
$$

Entretanto, se $X^{a}=\dot{\gamma}^{a}(\lambda)$ é nulo em relação a $g$ (e, portanto, $\left.\bar{g}\right)$, onde $\lambda$ é a parametrização afim original de $\gamma$, escolhendo $\bar{\lambda}=e^{2 \log z(\gamma(\lambda))} \lambda=z^{2} \lambda$, segue que

$$
\frac{d^{2}}{d \bar{\lambda}^{2}} \gamma(\bar{\lambda})=0
$$

i.e., $\gamma$ é uma geodésica nula em relação a $\bar{g}$ a menos de reparametrização. As relações envolvendo os tensores de RIEMANN e RiCCi ficam (tomando-se $z^{-1} \nabla_{a} z=\nabla_{a} \log z$ )

$$
\begin{aligned}
(\mathrm{A} .27) \operatorname{Riem}(\bar{g})_{a b c}^{d}= & \operatorname{Riem}(g)_{a b c}^{d}-2 \nabla_{[a} C_{b] c}^{d}+2 C_{c[a}^{e} C_{b] e}^{d}= \\
= & \operatorname{Riem}(g)_{a b c}^{d}+2 \delta_{[a}^{d} \nabla_{b]} \nabla_{c} \log z-2 g^{d e} g_{c[a} \nabla_{b]} \nabla_{e} \log z+ \\
& +2\left(\nabla_{[a} \log z\right) \delta_{b]}^{d} \nabla_{c} \log z-2\left(\nabla_{[a} \log z\right) g_{b] c} g^{d f} \nabla_{f} \log z+ \\
& -2 g_{c[a} \delta_{b]}^{d} g^{e f}\left(\nabla_{e} \log z\right) \nabla_{f} \log z
\end{aligned}
$$

e (contraindo-se os índices $d$ e $b$ em (A.23))

$$
\begin{aligned}
\operatorname{Ric}(\bar{g})_{a c}= & \operatorname{Ric}(g)_{a c}-\frac{d-2}{z} \nabla_{a} \nabla_{c} z-\frac{1}{z} g_{a c} g^{d e} \nabla_{d} \nabla_{e} z+ \\
& +2 \frac{d-2}{z^{2}}\left(\nabla_{a} z\right) \nabla_{c} z-\frac{d-3}{z^{2}} g_{a c} g^{d e}\left(\nabla_{d} z\right) \nabla_{e} z .
\end{aligned}
$$

É conveniente termos à disposição a forma inversa de (A.28), empregada no Capítulo 2. Invertendo os papéis de $g$ e $\bar{g}$ e tomando $z \mapsto z^{-1}$, chegamos em

$$
\operatorname{Ric}(g)_{a c}=\operatorname{Ric}(\bar{g})_{a c}+\frac{d-2}{z} \bar{\nabla}_{a} \bar{\nabla}_{c} z+\bar{g}_{a c} \bar{g}^{d e}\left(\frac{1}{z} \bar{\nabla}_{d} \bar{\nabla}_{e} z-\frac{d-1}{z^{2}}\left(\bar{\nabla}_{d} z\right) \bar{\nabla}_{e} z\right) .
$$

Segue das fórmulas (A.2), (A.27) e (A.28) que o tensor de WEYL é invariante por mudanças conformes da métrica, i.e.,

$$
C(\bar{g})_{a b c}^{d}=C(g)_{a b c}^{d}, \text { ou } C(\bar{g})_{a b c d}=z^{2} C(g)_{a b c d},
$$

onde subentende-se que o levantamento e abaixamento de índices de tensores construídos unicamente a partir de uma métrica $g$ devem ser feitos com $g$ (outros casos são tratados individualmente de acordo com o contexto).

Retornando a $\mathscr{I}$, apontamos que sempre é possível escolher $z$ tal que $\bar{\nabla}_{a} \bar{\nabla}_{b} z \Upsilon_{\mathscr{I}}=0$, sem restrições ao representante escolhido para a classe conforme de $\bar{g}^{(0)}$ (uma demonstração deste fato no caso em que $g$ satisfaz as equações de EINSTEIN sem matéria é apresentada na Seção 2.2). Costuma-se denotar o espaço-tempo $(\mathscr{M}, g)$ simplesmente por 
interior ("bulk"), e o infinito conforme correspondente $\left(\mathscr{I}, \bar{g}^{(0)}\right)$, por fronteira ("boundary").

Uma característica importante de mudanças conformes de uma métrica Lorentziana é que a estrutura causal é um invariante da estrutura conforme dessa métrica. Em particular, o infinito conforme também é denominado infinito nulo, devido à invariância do conceito de geodésicas nulas por mudanças conformes na métrica, como visto acima. Isto baseia-se na idéia de que geodésicas nulas e completas podem ter, do ponto de vista de $(\overline{\mathscr{M}}, \bar{g})$, extremos passados e futuros (no sentido dos "pontos ideais" de GEROCH, Kronheimer e Penrose [GKP72]) em $\mathscr{I}$. No entanto, note-se que $\mathscr{I}$ não precisa ser o "infinito" para toda geodésica nula completa. Um caso em que isso certamente ocorre é quando $(\mathscr{M}, g)$ é fortemente causal e $(\overline{\mathscr{M}}, \bar{g})$ é compacto (por exemplo: espaço-tempo de Minkowski); contudo, se $(\overline{\mathscr{M}}, \bar{g})$ não é compacto, pode acontecer que algumas geodésicas nulas não consigam chegar em $\mathscr{I}$. Isto motiva a seguinte

DefiniÇÃo A.4 Seja $(\mathscr{M}, g)$ um espaço-tempo d-dimensional com infinito conforme $\left(\mathscr{I}, \bar{g}^{(0)}\right)$. Dizemos que $(\mathscr{M}, g)$ é assintoticamente simples se toda geodésica nula em $(\mathscr{M}, g)$ possui uma única extensão a $(\overline{\mathscr{M}}, \bar{g})$ tal que $\mathscr{I}$ contém precisamente os dois extremos desta.

Obviamente, isto só é possível se $(\mathscr{M}, g)$ é nulamente geodesicamente completo. Na verdade, no caso em que $\left(\mathscr{I}, \bar{g}^{(0)}\right)$ é de tipo tempo (e, portanto, um espaço-tempo por si só), pode-se dizer mais, justificando o nome "assintoticamente simples":

Teorema A.1 Se $(\mathscr{M}, g)$ for assintoticamente simples com infinito conforme de tipo tempo, então ele é causalmente simples.

Prova. $\quad$ Primeiro, notar que se $p, q \in \mathscr{M}$ é tal que $p \nless \overline{\mathscr{M}} q$, então $p K_{\mathscr{M}} q$ (analogamente trocando-se o futuro pelo passado), pois uma curva de tipo tempo em $\overline{\mathscr{M}}$ ligando $p$ a $q$ pode sempre ser ligeiramente deformada de modo a resultar numa curva de tipo tempo contida em $\mathscr{M}$ e ligando $p$ a $q$. Agora, suponha que $p \in$ $\partial I^{-}(q, \mathscr{M})$ e $p \notin J^{-}(q, \mathscr{M})$. Pelo argumento acima, temos que $p \in \partial I^{-}(q, \overline{\mathscr{M}})$. Mais ainda, por hipótese, um gerador nulo $\gamma$ de $\partial I^{-}(q, \mathscr{M})$ precisa atingir seu extremo futuro no infinito sem cruzar $q$ antes disso. Seja $r$ tal extremo. Então, $r \in \partial I^{-}(q, \overline{\mathscr{M}})$ já que este conjunto é fechado. Uma vez que o infinito é totalmente geodésico[Rib07], $\gamma$ deve atingi-lo transversalmente e, portanto, qualquer extensão causal de $\gamma$ deve ser quebrada ${ }^{6}$. Logo, se estendermos $\gamma$ ligeiramente para o futuro por um segmento de gerador nulo $\gamma^{\prime}$ de $\partial I^{-}(q, \overline{\mathscr{M}})$ cruzando $r$ (digamos, ajustando o parâmetro afim $t$ de $\gamma^{\prime}$ igual a zero em $r$ e estendendo-o até $\left.t=\epsilon>0\right)$,

\footnotetext{
${ }^{6}$ Lembrar que um segmento de curva contínua é dito quebrado se ele for $\mathscr{C}^{\infty}$ por partes, mas não é $\mathscr{C}^{1}$ em um conjunto finito de pontos.
} 
então existe uma curva de tipo tempo em $\overline{\mathscr{M}}$ ligando $p$ a $\gamma^{\prime}(\epsilon)$ [HE73], o que viola a acronalidade de $\partial I^{-}(q, \overline{\mathscr{M}})$. Repetir o argumento trocando o futuro com o passado.

Um espaço-tempo assintoticamente simples não precisa, no entanto, ser globalmente hiperbólico - um exemplo típico é o espaço-tempo AdS. 


\section{APÊNDICE B}

\section{Elementos de álgebras de operadores}

O formalismo de álgebras de operadores é uma linguagem bastante poderosa para a discussão de aspectos estruturais da Física Quântica que são independentes da representação dos operadores em algum espaço de HiLBERT fixo, algo absolutamente fundamental no caso relativístico (i.e., Teoria Quântica de Campos) e, mais em geral, no estudo de sistemas com um número infinito de graus de liberdade (limites termodinâmicos em Mecânica Estatística Quântica, etc.).

C*-álgebras e álgebras de von Neumann (a serem vistas na Seção B.2) são adequadas à discussão de operadores limitados. Podemos, ao considerar operadores nãolimitados, fazer uso do cálculo funcional contínuo (teorema espectral) quando este se aplica e tratar apenas de funções limitadas desses operadores, evitando assim problemas técnicos referentes à determinação dos domínios. Ao discutirmos operadores de campo, bem como seu caráter distribucional, é todavia conveniente ter à nossa disposição um arcabouço algébrico para tratar tais operadores ilimitados diretamente, mas ainda assim de maneira independente de representações. Para tal, apresentamos um estudo razoavelmente detalhado de *álgebras na Seção B.1, antes de introduzirmos C*-álgebras ou álgebras de VON NEUMANN, para mostrar que vários resultados conhecidos no contexto de $\mathrm{C}^{*}$-álgebras continuam válidos ou sofrem apenas algumas modificações técnicas no contexto de *álgebras. Isto permite tratar os casos de álgebras de observáveis e de campos mais ou menos em paralelo. Para os últimos, mostraremos o formalismo proposto por Wightman, Borchers e Uhlmann na Seção B.3.

Limitamo-nos a demonstrar resultados cuja prova é simples do ponto de vista analítico e ao mesmo tempo instrutiva, e aqueles que são exigidos no presente trabalho numa forma não-padrão. Nossas referências principais para este Apêndice são [BR87, BR97], e empregamos como referências auxiliares [Mur90, SW00, Tak01, Tak03a]. Para aspectos funcional-analíticos, adotamos [RS80, Rud91]. 


\section{B.1 *-Álgebras}

Esta Seção é dedicada a aspectos puramente algébricos. Começaremos definindo a noção de *-álgebra e algumas de suas propriedades.

DEFINIÇÃo B.1 Uma *-álgebra (associativa) é um espaço vetorial $\mathfrak{F}$ sobre $\mathbb{C}$, dotado de um produto associativo e distributivo $x, y \mapsto x y$ que comuta com a multiplicação por escalares, e de uma operação de involução $x \mapsto a^{*}$ que satisfaz $x^{* *}=x,(x y)^{*}=y^{*} x^{*} e$ $(\alpha x+\beta y)^{*}=\bar{\alpha} x^{*}+\bar{\beta} y^{*}$, para todo $x, y \in \mathfrak{F}, \alpha, \beta \in \mathbb{C}$. Dizemos que $\mathfrak{F}$ é unital se existe uma identidade $\mathbb{1} \in \mathfrak{F}$ tal que $\mathbb{1} x=x \mathbb{1}=x$, para todo $x \in \mathfrak{F}$ (neste caso, $\mathbb{1}$ é único).

O centro de $\mathfrak{F}$ é dado por $\mathfrak{Z}(\mathfrak{F}) \doteq\{x \in \mathfrak{F}:[x, y]=0, \forall y \in \mathfrak{F}\}$, onde $[x, y] \doteq x y-y x$ denota o comutador de $x$ com $y$. Uma *álgebra $\mathfrak{F}$ é Abeliana se $\mathfrak{Z}(\mathfrak{F})=\mathfrak{F}$; no outro

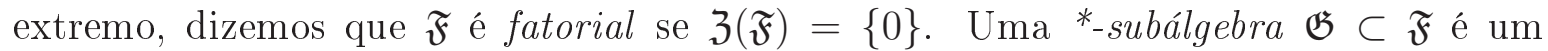
subespaço vetorial fechado por produto e involução. Exemplos de ${ }^{*}$-subálgebras de $\mathfrak{F}$ são $\mathfrak{Z}(\mathfrak{F})$ e, mais em geral, o comutante (relativo) ou normalizador de uma *-subálgebra $\mathfrak{G} \subset \mathfrak{F}$, dado por $\mathfrak{G}^{\prime}=\{x \in \mathfrak{F}:[x, y]=0, \forall y \in \mathfrak{G}\}$.

Podemos adjuntar uma identidade $\mathbb{1}$ a uma *álgebra não-unital $\mathfrak{F}$ da seguinte forma: seja $\widetilde{\mathfrak{F}}=\mathfrak{F} \oplus \mathbb{C} \mathbb{1} \ni(x, \lambda)$ com produto $(x, \lambda)(y, \mu) \doteq(x y+\mu x+\lambda y, \lambda \mu)$ e involução $(x, \lambda)^{*} \doteq\left(x^{*}, \bar{\lambda}\right)$. $\mathfrak{F}$ é naturalmente identificada com a ${ }^{*}$-subálgebra $(\mathfrak{F}, 0)$, e temos que $\mathbb{1}=(0,1)$.

Sejam $\mathfrak{F}, \mathfrak{G}$ *-álgebras. Um ${ }^{*}$-morfismo é uma aplicação linear $\pi: \mathfrak{F} \rightarrow \mathfrak{G}$ que preserva produto e involução. Se $\pi$ é respectivamente injetor, sobrejetor, bijetor, dizemos que $\pi$ é um *-monomorfismo, *-epimorfismo, ${ }^{*}$-isomorfismo - escrevemos $\mathfrak{F} \cong \mathfrak{G}$ se existe um *-isomorfismo de $\mathfrak{F}$ em $\mathfrak{G}$. Se $\mathfrak{F}=\mathfrak{G}$, dizemos que $\pi$ é um ${ }^{*}$-endomorfismo, e um *automorfismo se $\pi$, neste caso, for bijetor. Uma *-representação de $\mathfrak{F}$ num espaço de Hilbert $\mathscr{H}^{1}$ é um ${ }^{*}$-morfismo $\pi: \mathfrak{F} \rightarrow \mathscr{B}(\mathscr{H})$. Dizemos, então, que $\pi$ é fiel se $\pi$ for um *-monomorfismo, e irredutivel (abreviada irrep) se os únicos subespaços de $X$ invariantes por $\pi(\mathfrak{F})$ são $\{0\}$ e $X$ - pelo lema de SCHUR, $\pi$ é irredutível se e somente se $\{a \in \mathscr{B}(\mathscr{X}):[a, \pi(x)]=0, \forall x \in \mathfrak{F}\}=\{0\}$.

Um *-ideal de $\mathfrak{F}$ é um subespaço $\mathfrak{I} \subset \mathfrak{F}$ invariante por ${ }^{*}$, tal que $x \in \mathfrak{F}, y \in \mathfrak{I}$ implica

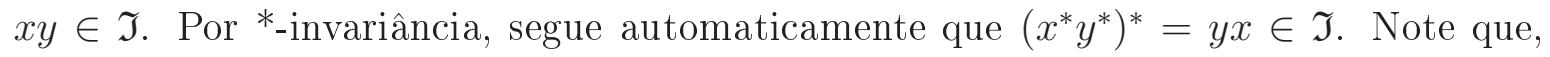
em contrapartida, ideais (não necessariamente *-invariantes) à esquerda de $\mathfrak{F}$ não o são necessariamente à direita, e vice-versa. $\mathfrak{F}$ é simples se não possui *-ideais não-triviais

\footnotetext{
${ }^{1}$ Demandamos uma estrutura de espaço de HilberT para evitar nos preocuparmos com a definição de involução em $\operatorname{End}_{\mathbb{C}} \mathscr{H}$. Uma definição de ${ }^{*}$-representação por elementos deste último (i.e., sem referência ao produto interno) envolveria termos à nossa disposição uma definição de involução, pelo menos na imagem da *álgebra em End $\mathbb{C} \mathscr{H}$.
} 
(i.e., diferentes de $\{0\}$ e $\mathfrak{F}$ ) - qualquer representação não-trivial $\pi$ de $\mathfrak{F}$ simples é fiel, pois $\operatorname{ker} \pi$ é um *-ideal de $\mathfrak{F}$.

Dado um *-ideal $\mathfrak{I} \subset \mathfrak{F}$, a ${ }^{*}$-álgebra quociente $\mathfrak{F} / \mathfrak{I}$ de $\mathfrak{F}$ por $\mathfrak{I}$ tem como elementos os "cosets" $[x]=x+\mathfrak{I} \subset \mathfrak{F}$, onde $[x]=[y]$ se $x-y \in \mathfrak{I}$. Por exemplo, se $\pi: \mathfrak{F} \rightarrow \mathfrak{G}$ é um *-morfismo, segue que $\mathfrak{F} / \operatorname{ker} \pi \cong \pi(\mathfrak{F}) \subset \mathfrak{G}$. O quociente possui a seguinte propriedade universal: dados uma ${ }^{*}$-álgebra $\mathfrak{G}$ e um ${ }^{*}$-morfismo $\rho: \mathfrak{F} \rightarrow \mathfrak{G}$ tal que $\operatorname{ker} \rho \subset \mathscr{I}$, existe um único *-morfismo $[\rho]: \mathfrak{F} / \mathfrak{I} \rightarrow \mathfrak{G}$ tal que $\rho(x)=[\rho]([x])$ para todo $x \in \mathfrak{F}$. Um *endomorfismo $\pi$ de $\mathfrak{F}$ induz um *-endomorfismo $[\pi]: \mathfrak{F} / \mathfrak{I} \ni[x] \mapsto \pi([x])=\pi(x)+\pi(\mathscr{I})$ se e somente se $\mathfrak{I}$ é invariante por $\pi$, i.e., $\pi(\mathfrak{I}) \subset \mathfrak{I}$. Em particular, $[\pi]$ é um *endomorfismo injetivo de $\mathfrak{F} / \mathfrak{I}$ se e somente se, em adição, $\operatorname{ker} \pi \subset \mathfrak{I}$ e $\pi(\mathfrak{I})$ supseteqq $\mathfrak{I}$.

Enunciamos a seguir quatro teoremas fundamentais sobre *-álgebras - os 3 últimos são os chamados Teoremas de *-Isomorfismo, análogos aos Teoremas de Isomorfismo para anéis e módulos (a incorporação da operação de involução * é imediata e pode ser checada diretamente).

Teorema B.1 (Teorema Fundamental de *-Morfismos) Sejam $\mathfrak{F}$ e $\mathfrak{G}$ duas *álgebras, e $\rho: \mathfrak{F} \rightarrow \mathfrak{G}$ um ${ }^{*}$-morfismo. Então o ${ }^{*}$-morfismo induzido $\tilde{\rho}: \mathfrak{F} /$ Ker $\rho \rightarrow$ $\rho(\mathfrak{F}) \subset \mathfrak{G}$ é um ${ }^{*}$-isomorfismo.

Prova. Imediato.

O Teorema B.1 pode ser generalizado, resultando no

Teorema B.2 (Primeiro Teorema de *-Isomorfismo ${ }^{2}$ ) Sejam $\mathfrak{F}$ e $\mathfrak{G}$ duas * álgebras, e $\rho: \mathfrak{F} \rightarrow \mathfrak{G}$ um ${ }^{*}$-epimorfismo. Então, $\mathfrak{H} \mapsto \rho(\mathfrak{H})$ estabelece uma correspondência biunivoca entre os subgrupos aditivos $\mathfrak{H} \supset$ Ker $\rho$ de $\mathfrak{F}$ e os subgrupos aditivos de $\mathfrak{G}$. Mediante esta correspondência, $\mathfrak{H}$ é uma ${ }^{*}$-subálgebra (resp. ${ }^{*}$-ideal) de $\mathfrak{F}$ se e somente se $\rho(\mathfrak{H})$ for uma ${ }^{*}$-subálgebra (resp. ${ }^{*}$-ideal) de $\mathfrak{G}$. Mais ainda, se $\mathfrak{I} \supset$ Ker $\rho$ é um *-ideal, então a aplicação

$$
x+\mathfrak{I} \mapsto \rho(x)+\rho(\mathfrak{I})
$$

é um *isomorfismo de $\mathfrak{F} / \mathfrak{I}$ em $\mathfrak{G} / \rho(\mathfrak{I})$.

Prova. A primeira assertiva segue imediatamente do Teorema B.2. O restante é demonstrado de maneira análoga ao caso de anéis - ver a demonstração do Teorema 2.6 de [Jac85], páginas 107-108.

\footnotetext{
${ }^{2}$ Não parece haver um acordo na literatura sobre a designação "Primeiro Teorema do Isomorfismo", nem para anéis e módulos, nem para grupos. Alguns autores atribuem este nome ao análogo do Teorema B.1, e outros, ao análogo do Teorema B.2, como fazemos aqui, pois o Teorema B.2 pode ser entendido, como dito acima, como uma generalização do Teorema B.1, e mais em acordo com o Segundo e Terceiro Teoremas de (*-)Isomorfismo.
} 
Teorema B.3 (Segundo Teorema de *-Isomorfismo) Seja $\mathfrak{F}$ uma *álgebra, $\mathfrak{G}$ uma ${ }^{*}$-subálgebra de $\mathfrak{F}$, e $\mathfrak{I}$ um ${ }^{*}$-ideal de $\mathfrak{F}$. Então $\mathfrak{G}+\mathfrak{I}$ é uma ${ }^{*}$-subálgebra de $\mathfrak{F}$, $\mathfrak{G} \cap \mathfrak{I}$ é um ${ }^{*}$-ideal de $\mathfrak{G}$, $\mathfrak{I}$ é um ${ }^{*}$-ideal de $\mathfrak{G}+\mathfrak{I}$, e $(\mathfrak{G}+\mathfrak{I}) / \mathfrak{I} \cong \mathfrak{G} /(\mathfrak{G} \cap \mathfrak{I})$.

Prova. Análogo ao caso de anéis - ver [Jac85], página 108.

Teorema B.4 (Terceiro Teorema De *-Isomorfismo) Seja $\mathfrak{F}$ uma *álgebra, $e$ $\mathfrak{I} \subset \mathfrak{J}$ dois ${ }^{*}$-ideais de $\mathfrak{F}$. Então $\mathfrak{I}$ é um ${ }^{*}$-ideal de $\mathfrak{J}$, e $\mathfrak{F} / \mathfrak{J} \cong(\mathfrak{F} / \mathfrak{I}) /(\mathfrak{J} / \mathfrak{I})$.

Prova. Segue imediatamente do Teorema B.2.

A demonstração dos análogos dos Teoremas B.2 e B.3 para anéis consistem tão somente na construção dos isomorfismos e checagem explícita das propriedades relevantes.

OBSERVAÇÃo B.2 No caso de *álgebras topológicas, em particular os exemplos a serem discutidos ao longo deste Apêndice, os Teoremas de B.1 a B.4 valem não só em nivel algébrico mas também em nivel topológico, contanto que se adicione o adjetivo "fechado" (nas respectivas topologias) aos termos "subgrupo", "*-subálgebra" e "*-ideal" - a continuidade dos *-morfismos é automática em todos os casos, em virtude da propriedade $C^{*}$ das (semi)normas (ver as Definiçôes B.3 e B.8). Neste caso, dada uma (semi)norma \|.\| numa ${ }^{*}$ álgebra $\mathfrak{F}$, a (semi)norma correspondente na ${ }^{*}$-álgebra quociente $\mathfrak{F} / \mathfrak{I}$, onde $\mathfrak{I} \subset \mathfrak{F}$ é um ${ }^{*}$-ideal, é dada por

$$
\mathfrak{F} / \mathfrak{I} \ni[x] \mapsto\|[x]\| \doteq \inf _{y \in \mathfrak{I}}\|x+y\| .
$$

Completeza desta norma (ou familia separante de seminormas) pode ser checada diretamente; para a verificaçãa da propriedade $C^{*}$ no caso de $C^{*}$-álgebras, ver a Proposição 2.2.19 de [BR87].

Um estado $\omega$ sobre uma *-álgebra unital $\mathfrak{F}$ é um funcional linear positivo sobre $\mathfrak{F}$, i.e., $\omega\left(x^{*} x\right) \geq 0$ para todo $x \in \mathfrak{F}$, que satisfaz $\omega(\mathbb{1})=1 .^{3}$ Dizemos que $\omega$ é fiel se $\omega\left(x^{*} x\right)>0$ para todo $x \neq 0$ (a razão do nome virá em breve). O conjunto dos estados sobre $\mathfrak{F}$ é convexo: se $\omega_{1}$ e $\omega_{2}$ são estados, então $\lambda \omega_{1}+(1-\lambda) \omega_{2}$ também o é, para todo $\lambda \in(0,1)$. Se $\omega$ não admite uma decomposição $\omega=\lambda \omega_{1}+(1-\lambda) \omega_{2}$ para $\omega_{1}, \omega_{2} \neq \omega$, $\lambda \in(0,1)$, dizemos que $\omega$ é puro (equivalentemente, $\omega$ é puro se não pode ser escrito como a soma de dois funcionais lineares positivos $\left.\phi_{1}, \phi_{2} \notin \mathbb{R}_{+} \omega\right)$. Caso contrário, dizemos que $\omega$ é misto. Denotamos o conjunto de todos os estados sobre $\mathfrak{F}$ por $\mathscr{S}_{\mathfrak{F}}$.

A positividade de $\omega$ leva à seguinte propriedade crucial:

\footnotetext{
${ }^{3}$ Se há uma topologia em $\mathfrak{F}$ à nossa disposição, podemos definir a condição de normalização de um estado mesmo na ausência de identidade.
} 
Lema B.5 (Desigualdade De Cauchy-Schwarz) Seja $\phi$ um funcional linear positivo sobre uma *álgebra (não necessariamente unital) $\mathfrak{F}$. Então, (i) $\phi\left(x^{*} y\right)=\overline{\phi\left(y^{*} x\right)}$ (em particular, se $\mathfrak{F}$ é unital, $\left.\phi\left(x^{*}\right)=\overline{\phi(x)}\right)$ e $($ ii $)\left|\phi\left(x^{*} y\right)\right|^{2} \leq \phi\left(x^{*} x\right) \phi\left(y^{*} y\right)$, para todo $x, y \in \mathfrak{F}$ (em particular, se $\mathfrak{F}$ é unital, $|\phi(x)|^{2} \leq \phi(\mathbb{1}) \phi\left(x^{*} x\right)$ ).

Prova (Esboço). Para todo $\lambda \in \mathbb{C}$, temos que $\phi\left((\lambda x+y)^{*}(\lambda x+y)\right) \geq 0$. Por linearidade,

$$
|\lambda|^{2} \phi\left(x^{*} x\right)+\bar{\lambda} \phi\left(x^{*} y\right)+\lambda \phi\left(y^{*} x\right)+\phi\left(y^{*} y\right) \geq 0 .
$$

A realidade do primeiro membro da desigualdade acima implica (i), de onde segue que podemos tomar agora $\lambda \in \mathbb{R}$. Escolhendo apropriadamente um intervalo para $\lambda$, prova-se (ii).

De posse do Lema B.5, podemos facilmente construir uma representação de $\mathfrak{F}$ por operadores lineares densamente definidos num espaço de HiLBERT $\mathscr{H}_{\phi}$ naturalmente associado a um funcional linear positivo $\phi$. Primeiro, notar que o aniquilador Ann $\phi$ de $\phi$, dado por $\operatorname{Ann} \phi=\left\{x \in \mathfrak{F}: \phi\left(x^{*} x\right)=0\right\}$, é um ideal à esquerda de $\mathfrak{F}$. De fato, Ann $\phi$ é um subespaço vetorial de $\mathfrak{F}$, devido à linearidade de $\phi$ e ao Lema B.5. Mais ainda, dados $x \in \operatorname{Ann} \phi, y \in \mathfrak{F}$, temos que $\phi\left((y x)^{*} y x\right)^{2} \leq \phi\left(x^{*} x\right) \phi\left(\left(x^{*} y^{*} y\right)\left(y^{*} y x\right)\right)=0$. Considere agora o espaço quociente $\mathfrak{F} / \operatorname{Ann} \phi \ni[x],[y]$, que admite o produto escalar dado por $\langle[x],[y]\rangle \doteq \phi\left(x^{*} y\right)$. Completando $\mathfrak{F} / \operatorname{Ann} \phi \ni[x],[y]$ com respeito à norma $\|$. associada a $\langle.,$.$\rangle , obtemos o espaço de Hilbert \mathscr{H}_{\phi} \doteq \overline{(\mathfrak{F} / \operatorname{Ann} \phi)^{\|\cdot\|}}$.

Consideremos o operador $\tilde{\pi}_{\phi}(x)$ associado a $x$, definido no subespaço linear denso $\mathfrak{F} / \operatorname{Ann} \phi$ por $\tilde{\pi}_{\phi}(x)[y] \doteq[x y]$. Como o adjunto de $\tilde{\pi}_{\phi}(x)$ é dado por $\tilde{\pi}_{\phi}(x)^{*} \uparrow_{\mathfrak{F} / \text { Ann } \phi}=$ $\tilde{\pi}_{\phi}\left(x^{*}\right)$ e ambos são densamente definidos, segue que $\tilde{\pi}_{\phi}(x)$ é fechável [RS80], com fecho $\overline{\pi_{\phi}(x)}=\pi_{\phi}(x)^{* *} \subset \pi_{\phi}\left(x^{*}\right)^{*}$ (lembrar que, dados operadores lineares densamente definidos $A, B$ com respectivos domínios $D(A), D(B), A \subset B$ denota que $B$ é uma extensão de $A$, i.e., $D(B) \supset D(A)$ e $\left.B \uparrow_{D(A)}=A\right) \operatorname{Logo}, \pi_{\phi}(x)=\overline{\pi_{\phi}(x)}=\pi_{\phi}(x)^{* *}$ define uma representação de $\mathfrak{F}$ por operadores lineares fechados e densamente definidos em $\mathscr{H}_{\phi}$. O operador anti-linear densamente definido dado por $S_{0}:[x] \mapsto[x *]$ será crucial no desenvolvimento da teoria modular de TOMITA-TAKESAKI mais adiante.

Note que a representação $\pi_{\phi}$ é fiel se e somente se $\phi\left(x^{*} x\right)>0$ para todo $x \neq 0$. Se $\mathfrak{F}$ é unital e $\omega$ é um estado, portanto, $\phi_{\omega}$ é fiel se e somente se $\omega$ é fiel, justificando o nome que demos. Para $\mathfrak{F}$ unital, há ainda um elemento especial de $\mathscr{H}_{\omega}$, dado por $\Omega=[\mathbb{1}]$. Obviamente, $\|\Omega\|=1$ se $\omega$ é um estado; mais importante, segue que o conjunto $\pi_{\omega}(\mathfrak{F}) \Omega=\mathfrak{F} /$ Ann $\phi$ é denso em $\mathscr{H}_{\omega}$, i.e., $\Omega$ é um vetor cíclico para $\pi_{\omega}(\mathfrak{F}) .{ }^{4}$ Neste caso, a representação construída acima, denotada pela tripla $\left(\mathscr{H}_{\omega}, \pi_{\omega}, \Omega\right)$, é denominada

\footnotetext{
${ }^{4}$ No caso de $C^{*}$-álgebras, é possível construir um vetor cíclico mesmo na ausência de uma identidade [Mur90].
} 
representação cíclica ou representação Wightman-GNS de $\mathfrak{F}$ associada ao funcional linear positivo $\omega .{ }^{5} \pi_{\omega}$ é fiel se e somente se $\Omega$, além de cíclico, for separante para $\mathfrak{F}$, i.e., $\pi_{\omega}(x) \Omega=0 \Rightarrow x=0$.

A representação Wightman-GNS de uma *-álgebra unital $\mathfrak{F}$ associada a um funcional linear positivo $\phi$ é única no seguinte sentido: seja $\pi$ uma representação de $\mathfrak{F}$ por operadores lineares densamente definidos e fechados num espaço de HilBERT $\mathscr{H}$, tal que:

- Existe um domínio $\mathscr{D}$, comum a todos $\pi(x)$ 's, denso em $\mathscr{H}$ e tal que $\pi \mathfrak{F} \mathscr{D} \subset \mathscr{D}$;

- Existe um vetor $\Phi^{\prime} \in \mathscr{D}$ cíclico para $\pi(\mathfrak{F})$ tal que $\pi(\mathfrak{F}) \Phi^{\prime}=\mathscr{D}$ e $\left\langle\Phi^{\prime}, \pi(x) \Phi^{\prime}\right\rangle=$ $\phi(x)$ para todo $x \in \mathfrak{F}$.

Podemos então definir o operador linear $U: \mathfrak{F} / \operatorname{Ann} \phi \rightarrow \mathscr{H}$ como $U[x] \doteq \pi(x) \Phi^{\prime}$. $U$ é densamente definido e satisfaz $\langle U[x], U[y]\rangle=\phi\left(x^{*} y\right)=\left\langle\pi(x) \Phi^{\prime}, \pi(y) \Phi^{\prime}\right\rangle$ para todo $x, y \in \mathfrak{F}$, ou seja, $U$ é uma isometria. Pelo Teorema BLT [RS80], $U$ se estende unicamente a uma isometria $U$ de $\mathscr{H}_{\phi}$ em $\mathscr{H}$, injetora e com imagem densamente definida. Invocando uma vez mais o Teorema BLT, temos que $U$ é uma bijeção, e sua inversa $U^{-} 1=U^{*}$ também é uma isometria. Ou seja, as representações $\pi_{\phi}$ e $\pi$ são unitariamente equivalentes. ${ }^{6}$ Outra conseqüência importante é que, se $\Phi=[\mathbb{1}]$ é o vetor cíclico de $\pi_{\phi}$, então $U \Phi=\Phi^{\prime}$. Uma conseqüência imediata deste fato, que podemos declarar como um Corolário, é:

Corolário B.6 Se a é um *automorfismo de uma *álgebra unital $\mathfrak{F}$ e $\phi$, um funcional linear positivo tal que $\phi \circ \alpha=\phi$, então existe um único operador unitário $U_{\alpha}$ em $\mathscr{H}_{\phi}$ tal que $\pi_{\phi}(\alpha(x))=U \pi_{\phi}(x) U^{-1}$.

Ou seja, uma condição suficiente para que um *-automorfismo $\alpha$ seja unitariamente implementável em $\mathscr{H}_{\phi}$ é que $\phi$ seja invariante pela ação de $\alpha$. Este é o principal mecanismo de implementação de simetrias na teoria quântica. Por fim, há uma relação estreita entre a pureza de um estado $\omega$ e uma contraparte "fraca" da noção de irredutibilidade para $\pi_{\omega}$ :

${ }^{5}$ GNS atende pelos nomes de GEL'FAnd e NAIMARK [GN43] e SEgAL [Seg47], que propuseram a construção acima no caso em que $\mathfrak{F}$ é uma $C^{*}$-álgebra - ver Seção B.2. O nome de Wightman vem anexo pois a construção acima é a parte central de seu Teorema de reconstrução de campos quânticos a partir de uma hierarquia de funções de $n$ pontos [SW00]. Teremos mais a dizer a respeito na Seção B.3.

${ }^{6}$ No caso em que $\mathfrak{F}$ é uma $C^{*}$-álgebra, a unicidade da representação GNS, dada por $U$, permanece mesmo se $\mathfrak{F}$ não possui identidade, mas neste caso o vetor cíclico de $\pi_{\phi}$ não necessariamente pertence a $\mathfrak{F} /$ Ann $\phi$, e devemos impor analogamente apenas que $\pi(\mathfrak{F}) \Phi^{\prime} \subset \mathscr{D}$ é denso em $\mathscr{H}$. 
Teorema B.7 Seja $\omega$ um estado sobre uma *álgebra unital $\mathfrak{F}$. Defina o comutante fraco

$$
\pi_{\omega}(\mathfrak{F})_{w}^{\prime} \doteq\left\{T \in \mathscr{B}\left(\mathscr{H}_{\omega}\right):\left\langle T^{*}[x], \pi_{\omega}(y)[z]\right\rangle=\left\langle\pi_{\omega}\left(y^{*}\right)[x], T[z]\right\rangle, \forall x, y, z \in \mathfrak{F}\right\}
$$

de $\pi_{\omega}(\mathfrak{F}) ; \pi_{\omega}(\mathfrak{F})_{w}^{\prime}$ é um subespaço linear ${ }^{*}$-invariante de $\mathscr{B}\left(\mathscr{H}_{\omega}\right)$. Então, $\pi_{\omega}(\mathfrak{F})_{w}^{\prime}=\mathbb{C} \mathbb{1}$ se e somente se $\omega$ for puro. Mais ainda, existe uma correspondência biunívoca $\omega_{T} \leftrightarrow T$ entre funcionais lineares positivos $\omega_{T}$ satisfazendo $\omega_{T} \leq \omega$ e elementos positivos $T$ de $p i_{\omega}(\mathfrak{F})_{w}^{\prime}$ satisfazendo $\|T\| \leq 1$.

Prova. $\quad(\Rightarrow)$ Suponha que $\omega$ é misto. Então existe um funcional linear positivo $\omega^{\prime}$ tal que $\omega^{\prime}\left(x^{*} x\right) \leq \omega\left(x^{*} x\right)$, para todo $x$, e tal que $\omega^{\prime}$ não é um múltiplo de $\omega$. Aplicando a desigualdade de Cauchy-Schwarz, temos que $\left|\omega^{\prime}\left(x^{*} y\right)\right|^{2} \leq$ $\left\|\pi_{\omega}(x) \Omega\right\|^{2}\left\|\pi_{\omega}(y)\right\|^{2}$. Portanto, $\pi_{\omega}(x) \Omega \times \pi_{\omega}(y) \Omega \mapsto \omega^{\prime}\left(x^{*} y\right)$ define uma forma sesquilinear limitada e densamente definida em $\mathscr{H}_{\omega}$. Assim, existe um único operador limitado $T$ em $\mathscr{H}_{\omega}$ tal que $\left\langle\pi_{\omega}(x) \Omega, T \pi_{\omega}(y) \Omega\right\rangle=\omega^{\prime}\left(x^{*} y\right)$, e $T$ não é um múltiplo de $\mathbb{1}$. Mais ainda, $0 \leq\left\langle\pi_{\omega}(x) \Omega, T \pi_{\omega}(x) \Omega\right\rangle \leq\left\|\pi_{\omega}(x)\right\|^{2}$, e, assim, $0 \leq T \leq \mathbb{1}$. Mas, neste caso,

$$
\left\langle\pi_{\omega}(x) \Omega, T \pi_{\omega}(y) \pi_{\omega}(x) \Omega\right\rangle=\omega^{\prime}\left(x^{*} y z\right)=\omega^{\prime}\left(\left(y^{*} x\right)^{*} z\right)=\left\langle\pi_{\omega}\left(y^{*}\right) \pi_{\omega}(x) \Omega, T \pi_{\omega}(z) \Omega\right\rangle
$$

para todo $x, y, z \in \mathfrak{F}$, provando que $T$ é um elemento não-trivial de $\pi_{\omega}(\mathfrak{F})_{w}^{\prime}$.

$(\Leftarrow)$ Suponha que $T \in \pi_{\omega}(\mathfrak{F})_{w}^{\prime}, T \notin \mathbb{C} \mathbb{1}$. Então, $T^{*}$ e, portanto, $T+T^{*}$ pertencem a $\pi_{\omega}(\mathfrak{F})_{w}^{\prime}$, sendo que o último é auto-adjunto e não-trivial. Defina $S \doteq \lambda(2 \| T+$ $\left.T^{*} \| \mathbb{1}+\left(T+T^{*}\right)\right) \in \pi_{\omega}(\mathfrak{F})_{w}^{\prime}$, onde $\lambda>0$ é escolhido tal que $\|S\|<1$. Então, $0<S<\mathbb{1}$, e portanto existe um projetor espectral $P$ de $S$ tal que $0<P<\mathbb{1}$ e $P \in \pi_{\omega}(\mathfrak{F})_{w}^{\prime}$. Considere o funcional linear $\omega^{\prime}(x)=\left\langle P \Omega, \pi_{\omega}(x) \Omega\right\rangle$. $\omega^{\prime}$ é positivo, pois

$\omega^{\prime}\left(x^{*} x\right)=\left\langle P \Omega, \pi_{\omega}\left(x^{*}\right) \pi_{\omega}(x) \Omega\right\rangle=\left\langle\pi_{\omega}(x) \Omega, P \pi_{\omega}(x) \Omega\right\rangle=\left\langle P \pi_{\omega}(x) \Omega, \pi_{\omega}(x) \Omega\right\rangle \geq 0$.

Finalmente,

$$
\omega\left(x^{*} x\right)-\omega^{\prime}\left(x^{*} x\right)=\left\langle\pi_{\omega}(x) \Omega, P \pi_{\omega}(x) \Omega\right\rangle \geq 0,
$$

mostrando que $\omega$ é misto.

A assertiva final segue automaticamente dos argumentos acima.

\section{B.2 C*-álgebras e álgebras de VON NEUMANN}

\section{B.2.1 C*-álgebras}

Vamos agora dar uma condição suficiente para que os operadores fechados $\pi_{\phi}(x)$ construídos por meio da representação WiGHTMAN-GNS na Seção B.1 sejam limitados. 
Se pudermos encontrar para todo $x$ um $C(x)>0$ tal que $\phi\left((x a)^{*} x a\right) \leq C(x)^{2} \phi\left(a^{*} a\right)$ para todo $a \in \mathfrak{F}$ e todo funcional linear positivo $\phi$ (i.e., $C$ não depende de $\phi$ ), então segue que $\left\|\pi_{\phi}(x)[a]\right\| \leq C(x)\|[a]\|$. Portanto, pelo teorema $\operatorname{BLT}, \pi_{\phi}(x)$ admite uma única extensão a $\mathscr{H}_{\phi}$, e $\left\|\pi_{\phi}(x) \Psi\right\| \leq C(x)\|\Psi\|$ para todo $\Psi \in \mathscr{H}_{\phi}$. Neste caso, $\pi_{\phi}\left(x^{*}\right)=\pi_{\phi}(x)^{*}$ e, assim, $\pi_{\phi}$ define uma ${ }^{*}$-representação de $\mathfrak{F}$ por operadores limitados em $\mathscr{H}_{\phi}$.

A maneira mais óbvia de obter $C$ como no parágrafo anterior é impor uma norma $\|$.$\| em \mathfrak{F}$ tal que o produto e a involução de $\mathfrak{F}$ sejam contínuas com respeito a esta norma, definindo uma *álgebra normada. Em particular, podemos escolher uma norma equivalente à original tal que $\|x y\| \leq\|x\|\|y\| \mathrm{e}$, portanto, $\|\mathbb{1}\|=1$ se $\mathfrak{F}$ for unital (tal propriedade está subentendida ao longo de todo o presente trabalho). Veremos que $C(x) \doteq\|x\|$ satisfaz a condição no parágrafo anterior se:

1. $\mathfrak{F}$ é completa com respeito a $\|$.$\| (dizemos então que \mathfrak{F}$ é uma *-álgebra de BA$\mathrm{NACH}), \mathrm{e}$

2. $\left\|x^{*} x\right\|=\|x\|^{2}$. Neste caso, segue que $\|x\|^{2},\left\|x^{*}\right\|^{2} \leq\|x\| \cdot\left\|x^{*}\right\|$ e, portanto, $\|x\|=$ $\left\|x^{*}\right\|$.

DEFiniçÃo B.3 Uma $\mathrm{C}^{*}$-álgebra $\mathfrak{A}$ é uma *álgebra de BANACH cuja norma satisfaz a condição $\mathrm{C}^{*}\left\|x^{*} x\right\|=\|x\|^{2} \cdot{ }^{7}$

Um exemplo típico de $\mathrm{C}^{*}$-álgebra é a *álgebra unital $\mathscr{B}(\mathscr{H})$ de operadores limitados num espaço de Hilbert complexo $\mathscr{H}$. Vamos citar agora dois resultados de uso freqüente, que mostram outrossim a força da Definição B.3.

ProposiçÃo B.8 Todo *-morfismo $\rho$ de uma *álgebra de BAnACH unital $\mathfrak{B}$ numa $C^{*}$-álgebra $\mathfrak{C}$ satisfaz $\|\rho(x)\| \leq\|x\|$ para todo $x \in \mathfrak{B}$. Se $\mathfrak{B}$ é uma $C^{*}$-álgebra, então $\rho(\mathfrak{B})$ é uma $C^{*}$-subálgebra de $\mathfrak{C}, e\|\rho(x)\|=\|x\|$ para todo $x$ se e somente se $\rho$ é um *-monomorfismo.

Prova. Ver as Proposições 2.3.1 e 2.3.3 de [BR87].

Proposição B.9 Todo funcional linear positivo $\phi$ sobre uma $C^{*}$-álgebra $\mathfrak{A}$ (não necessariamente unital) satisfaz as seguintes propriedades:

(i) $\phi\left(x^{*}\right)=\overline{\phi(x)}$;

(ii) $|\phi(x)|^{2} \leq\|\phi\| \phi\left(x^{*} x\right)$ (em particular, $\|\phi\|=\phi(\mathbb{1})$ se $\mathfrak{A}$ for unital);

$7 \mathrm{Na}$ verdade, não é necessário impor a condição $\|x y\| \leq\|x\|\|y\|$ ou mesmo a continuidade do produto e da involução como axiomas definindo uma $C^{*}$-álgebra. ARAKI e ElliotT [AE73] mostraram que a primeira condição, e, portanto, a condição $\|x\|=\left\|x^{*}\right\|$ seguem dos demais axiomas (completeza da norma, estrutura de *-álgebra e a condição $\mathrm{C}^{*}$ ). 
(iii) $\left|\phi\left(x^{*} y x\right)\right| \leq \phi\left(x^{*} x\right)\|y\|$;

(iv) $\|\phi\|=\sup \left\{\phi\left(x^{*} x\right):\|x\|=1\right\}$.

Prova. Ver a Proposição 2.3.11 de [BR87].

Segue da Proposição B.9(iii) que $\phi\left((x y)^{*} x y\right) \leq\|x\|^{2} \phi\left(y^{*} y\right)$. Portanto, $C(x)=\|x\|$ satisfaz a estimativa no primeiro parágrafo desta Seção, e a representação GNS $\pi_{\phi}$ é, assim, uma *-representação de $\mathfrak{A}$ por elementos da $\mathrm{C}^{*}$-álgebra $\mathscr{B}\left(\mathscr{H}_{\phi}\right)$. O exemplo aparentemente particular de $\mathrm{C}^{*}$-álgebra dado por $\pi_{\phi}(\mathfrak{A})$, na verdade, é o mais geral possível, pois GEL'FAND e NAIMARK provaram [GN43] que toda $\mathrm{C}^{*}$-álgebra é *-isomorfa a uma $\mathrm{C}^{*}$-subálgebra de $\mathscr{B}(\mathscr{H})$ para algum espaço de HilberT $\mathscr{H}$. Mais precisamente, a representação universal

$$
\mathscr{H}_{u}=\bigoplus_{\omega \in \mathscr{S}_{\mathfrak{A}}} \mathscr{H}_{\omega}, \pi_{u}=\bigoplus_{\omega \in \mathscr{S}_{\mathfrak{A}}} \pi_{\omega}
$$

é fiel, pois pode-se provar [BR87] que, dado $x \in \mathscr{A}$, existe um estado puro $\omega_{x}$ tal que $\omega_{x}\left(x^{*} x\right)=\|x\|^{2}$. Como $\pi_{\omega_{x}}$ neste caso é irredutível (ver próximo parágrafo), segue que $\pi_{u}$ possui, dentre suas subrepresentações irredutíveis, $\pi_{\omega_{x}}$ para todo $x$. Portanto, $\pi_{u}(x) \neq 0$ para todo $x \neq 0$.

Por fim, consideremos a seguinte classe de elementos de uma $\mathrm{C}^{*}$-álgebra $\mathfrak{A}$. Dizemos que $x \in \mathfrak{A}$ é positivo se for auto-adjunto e seu espectro $\sigma(x)$ satisfaz $\sigma(x) \subset \overline{\mathbb{R}}_{+}$, ou, equivalentemente, pode ser escrito como $x=y^{*} y, y \in \mathfrak{A}^{8}{ }^{8}$ Os elementos positivos de $\mathfrak{A}$ formam um cone convexo $\mathfrak{A}_{+}$que satisfaz $\mathfrak{A}_{+} \cap\left(-\mathfrak{A}_{+}\right)=\{0\}$, e todo elemento autoadjunto $x \in \mathfrak{A}$ pode ser escrito como a decomposição ortogonal $x=x_{+}-x_{-}$, onde $x_{ \pm}=\frac{1}{2}(x \pm|x|) \in \mathfrak{A}_{+},|x|=\sqrt{x^{*} x}$ e $x_{+} x_{-}=0$. Podemos, assim, impor o seguinte ordenamento $\leq$ aos elementos auto-adjuntos de $\mathfrak{A}$, dado por $x \leq y \Leftrightarrow y-x \in \mathfrak{A}_{+}$. Vemos imediatamente que $A \leq B$ e $B \leq A$ implicam $A=B$, e, pela fórmula do raio espectral [RS80, Rud91], segue que $\mathfrak{A}_{+} \ni x \leq\|x\| \mathbb{1}$. Assim, quando nos referimos a subconjuntos (de)crescentes de $\mathfrak{A}_{+}$e a limites superiores, supremos, limites inferiores ou ínfimos de subconjuntos de $\mathfrak{A}_{+}$, será sempre em relação ao ordenamento $\leq$definido acima.

${ }^{8}$ À primeira vista, poderíamos definir positividade diretamente numa *álgebra na Seção B.1 usando esta condição. Evitamos esta terminologia explícita neste caso mais geral (exceto, é claro, na definição de funcional linear positivo e estado numa *álgebra), pois a representação WightMAN-GNS de um elemento "positivo" neste sentido define um operador fechado e simétrico, mas não necessariamente auto-adjunto. Assim, a noção intuitiva de operador positivo como referindo-se à nãonegatividade do espectro se perde para *álgebras, o que poderia levar a mal-entendidos perigosos. 


\section{B.2.2 Álgebras de VON NEUMANN}

Consideremos agora um estado $\omega$ sobre $\mathfrak{A}$. Neste caso, o comutante fraco $\pi_{\omega}(\mathfrak{A})_{w}^{\prime}$ é uma $\mathrm{C}^{*}$-álgebra, e coincide com o comutante $\pi_{\omega}(\mathfrak{A})^{\prime} \doteq\left\{T \in \mathscr{B}\left(\mathscr{H}_{\omega}\right):\left[T, \pi_{\omega}(A)\right] \stackrel{=}{\doteq}\right.$ $\left.T \pi_{\omega}(A)-\pi_{\omega}(A) T=0, \forall A \in \mathfrak{A}\right\}$ de $\pi_{\omega}(\mathfrak{A})$ em $\mathscr{B}\left(\mathscr{H}_{\omega}\right)$. Podemos então reforçar a conclusão do Teorema B.7: $\omega$ é puro se e somente se $\pi_{\omega}$ é irredutível. Mais ainda, $\pi_{\omega}(\mathfrak{A})^{\prime}$ é uma $\mathrm{C}^{*}$-álgebra de um tipo bastante particular, pois $\pi_{\omega}(\mathfrak{A})^{\prime}=\left(\pi_{\omega}(\mathfrak{A})^{\prime}\right)^{\prime \prime}$.

DefiniçÃo B.4 Seja uma $C^{*}$-álgebra unital $\Re$ concretamente realizada como uma $C^{*}$ subálgebra de $\mathscr{B}(\mathscr{H})$ para algum espaço de Hilbert $\mathscr{H}$. Dizemos que $\mathfrak{A}$ é uma álgebra de VON NEUMANN se $\mathfrak{R}=\mathfrak{R}^{\prime \prime}$.

Dizemos que uma álgebra de von Neumann $\mathfrak{R}$ fatorial, i.e., $\mathfrak{Z}(\mathfrak{F}) \doteq \mathfrak{F} \cap \mathfrak{F}^{\prime}=\mathbb{C} \mathbb{1}$, é simplesmente um fator. O Teorema do Duplo Comutante de von Neumann nos diz que uma *-subálgebra não-trivial $\mathfrak{R}$ de $\mathscr{B}(\mathscr{H})$ é uma álgebra de von NEumanN se e somente se $\mathfrak{R}$ fechada nas topologias forte, fraca e $\sigma$-fraca (também chamada ultrafraca) de $\mathscr{B}(\mathscr{H})$. Esta última é dada pelas seminormas $\sum_{i=1}^{\infty}\left|\left\langle\Psi_{i}, . \Phi_{i}\right\rangle\right|$, onde $\left\{\Psi_{i}\right\}_{i},\left\{\Phi_{i}\right\}_{i} \subset \mathscr{H}$ satisfazem $\sum_{i=1}^{\infty}\left\|\Psi_{i}\right\|^{2}, \sum_{i=1}^{\infty}\left\|\Phi_{i}\right\|^{2}<\infty$. Mais ainda, dada uma $\mathrm{C}^{*}$-subálgebra $\mathfrak{A} \in \mathscr{B}(\mathscr{H})$, temos que $\mathfrak{A}^{\prime \prime}$ é o fecho de $\mathfrak{A}$ nas topologias forte, fraca e $\sigma$-fraca. Citamos dois resultados acerca dessa última topologia:

Proposição B.10 Seja $\mathfrak{R}$ uma álgebra de von Neumann, e $\mathfrak{I}$ um ideal $\sigma$-fracamente fechado à esquerda e à direita em $\mathfrak{R}$. Então $\mathfrak{I}$ é um ${ }^{*}$-ideal, e existe uma projeção $E \in \mathfrak{Z}(\mathfrak{R})$ tal que $\mathfrak{I}=E \mathfrak{R}$.

Prova. Ver a Proposição 2.4.22 de [BR87].

Teorema B.11 Dadas duas álgebras de von Neumann $\mathfrak{R}$ e $\mathfrak{S}$, um ${ }^{*}$-morfismo de $\mathfrak{R}$ em $\mathfrak{S}$ é necessariamente $\sigma$-fracamente contínuo.

Prova. Ver o Teorema 2.4.23 de [BR87].

Se $\mathfrak{A}$ é uma $\mathrm{C}^{*}$-subálgebra de $\mathscr{B}(\mathscr{H})$, vê-se que $\mathfrak{A}$ é densa em $\mathfrak{A}^{\prime \prime}$ nas topologias forte, fraca e $\sigma$-fraca de $\mathscr{B}(\mathscr{H})$.

Note que, se um elemento $T \in \mathscr{B}(\mathscr{H})$ comuta $\operatorname{com} x=x^{*} \in \mathfrak{R}$, ele comuta com todas os projetores espectrais de $x$. Portanto, essas projeções pertencem a $\mathfrak{R}$. Utilizando a decomposição de um elemento de $\mathscr{B}(\mathscr{H})$ numa combinação linear de elementos auto-adjuntos, segue que as projeções de $\mathfrak{R}$ são densas em $\mathfrak{R}$ na norma; já utilizando-se a decomposição numa combinação linear de elementos unitários [RS80], mostra-se que $x \in \mathfrak{R}$ se e somente se a isometria parcial $u$ e o elemento positivo $|x|$ ocorrendo na decomposição polar $x=u|x|$ de $x$, bem como todos os projetores espectrais de $|x|$, pertencem a $\mathfrak{R}$ [BR87]. Mais em geral, dado um operador linear densamente definido 
e fechado $T$ em $\mathscr{H}$, dizemos que $T$ é afiliado a $\mathfrak{R}$ se $\mathfrak{R}^{\prime} D(T) \subseteq D(T)$ e $T x \supseteq x T$ para todo $x \in \mathfrak{R}^{\prime}$. Equivalentemente, $T$ é afiliado a $\mathfrak{R}$ se e somente se a isometria parcial $U$ e os projetores espectrais do operador positivo $|T|$ ocorrendo na decomposição polar $T=U|T|$ de $T$ pertencem a $\mathfrak{R}$.

Dada uma álgebra de von Neumann $\mathfrak{R}$, seu predual $\mathfrak{R}_{*}$ consiste dos funcionais $\sigma$ fracamente contínuos em $\mathfrak{R}$. Este é um subespaço de BANACH do dual $\mathfrak{R}^{*}$ do espaço de Banach $\mathfrak{R}$, sendo que este último coincide precisamente com o dual $\left(\mathfrak{R}_{*}\right)^{*}$ de $\mathfrak{R}_{*}$ [BR87]. O exemplo típico é $\mathscr{B}(\mathscr{H})$ ( $\mathscr{H}$ separável), cujo predual é dado pelos operadores "trace class" $\mathscr{L}_{1}(\mathscr{H})$, e o pareamento dual é dado pelo traço em $\mathscr{H}$. Temos, em geral, o seguinte resultado estrutural, devido a SAKAI:

Teorema B.12 (SAKai [Tak01]) Uma $C^{*}$-álgebra $\mathfrak{A}$ é *-isomorfa a uma álgebra de VOn NEUmann se e somente se for o dual de algum espaço de BANACH.

Devido ao Teorema B.12, é costume denominar tais álgebras de vON NEUmAnN "abstratas" por $W^{*}$-álgebras ( $W$ corresponde a "weak", denotando fecho na topologia fraca), mas aqui usaremos os dois termos alternadamente ao tratar de tais $\mathrm{C}^{*}$-álgebras. Vemos também que a topologia mais naturalmente associada a uma álgebra de VON Neumann é a topologia $\sigma$-fraca, e é a esta que, por exemplo, nos referimos ao empregar a versão "topologizada" dos Teoremas de B.1 a B.4 no contexto de W*-álgebras, conforme a Observação B.2. Contudo, conforme a Proposição 2.4.2 de [BR87], a operação de multiplicação é separadamente $\sigma$-fracamente contínua nas duas variáveis, mas $n \tilde{a} o$ é conjuntamente $\sigma$-fracamente contínua se a $\mathrm{W}^{*}$-álgebra em questão for fielmente realizada num espaço de HILBERT de dimensão infinita.

Dada uma $C^{*}$-álgebra $\mathscr{A}$ e um estado $\omega$, temos uma álgebra de von Neumann em $\mathscr{H}_{\omega}$ naturalmente associada a este par, dada por $\pi_{\omega}(\mathfrak{A})^{\prime \prime}$. Mais em geral, é possível mostrar que o duplo dual $\mathfrak{A}^{* *}$ de $\mathfrak{A}$ é uma $\mathrm{C}^{*}$-álgebra ${ }^{*}$-isomorfa a $\pi_{u}(\mathfrak{A})^{\prime \prime}$, denotada $W^{*}$-álgebra envolvente (universal) de $\mathfrak{A}$. Esta é a menor $W^{*}$-álgebra que contém $\mathfrak{A}$, e é *-isomorfa a $\pi_{\omega}(\mathfrak{A})^{\prime \prime}$ se $\omega$ for fiel. Um estado $\omega$ sobre uma álgebra de von NEUmanN $\mathfrak{R}$ sobre $\mathscr{H}$ é dito normal se existe um elemento "trace class" $\rho \in \mathscr{B}(\mathscr{H})$ tal que $\operatorname{Tr}(\rho)=1$ e $\omega(x)=\operatorname{Tr}(\rho x)$. Todo estado $\omega$ sobre uma $\mathrm{C}^{*}$-álgebra $\mathfrak{A}$ admite uma única extensão normal a $\mathfrak{A}^{* *}$, que é fiel se e somente se $\omega$ o é [Tak01].

Estados normais possuem a seguinte caracterização:

Teorema B.13 Seja $\omega$ um estado sobre uma álgebra de von Neumann $\mathfrak{R}$ no espaço de Hilbert $\mathfrak{H}$. Então, são equivalentes:

(i) $\omega$ é normal; 
(ii) $\omega$ é $\sigma$-fracamente contínuo;

(iii) Dada qualquer rede crescente $\left\{A_{\alpha}\right\} \subset \mathfrak{R}_{+}$limitada superiormente, segue que $\omega\left(\sup _{\alpha} A_{\alpha}\right)=\sup _{\alpha} \omega\left(A_{\alpha}\right)$.

Prova. Ver o Teorema 2.4.21 de [BR87].

Cabe aqui um comentário sobre a condição (iii) do Teorema B.13. Não é óbvio que,

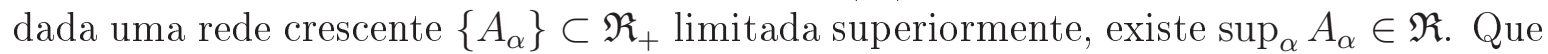
isto é verdade, pode ser visto a seguir: seja $\mathfrak{R}_{\alpha}$ o fecho de $\left\{A_{\beta}: \beta>\alpha\right\}$ na topologia fraca de $\mathscr{B}(\mathscr{H})$. Como a bola unitária fechada $\mathscr{B}(\mathscr{H})_{1}$ deste último é fracamente compacta, ${ }^{9}$ segue da estrutura de cone de $\mathfrak{R}_{+}$que existe $A \in \cap_{\alpha} \mathfrak{R}_{\alpha}$. Para todo $A_{\alpha}$, $\left\{B \in \mathscr{B}(\mathscr{H})_{+}: B \geq A_{\alpha}\right\}$ é $\sigma$-fracamente fechado e contém $\mathfrak{R}_{\alpha}$, portanto $A \geq A_{\alpha}$ para todo $\alpha$. Qualquer elemento $B$ majorando $\left\{A_{\alpha}\right\}$ majoriza seu fecho fraco e, assim, $B \geq A$. Logo, $A=\sup _{\alpha} A_{\alpha}$. Mais ainda, $A_{\alpha} \rightarrow A$ na topologia $\sigma$-fraca, pois esta coincide com a topologia fraca em $\mathscr{B}(\mathscr{H})_{1}$ [BR87], estabelecendo assim (ii) $\Rightarrow$ (iii) no Teorema B.13. Dizemos em geral que um funcional linear positivo $\phi$ numa álgebra de Von Neumann $\mathfrak{R}$ é normal se satisfaz a condição (iii) do Teorema B.13. ${ }^{10}$

Concluímos esta seção com a classificação de álgebras de vON NEUmann introduzida por MURRAY e VON NEUMANN: seja

$$
\mathscr{P}(\mathfrak{R}) \doteq\left\{E \in \mathfrak{R}: E=E^{*}=E^{2}\right\}
$$

o conjunto das projeções da álgebra de vOn Neumann $\mathfrak{R}$. $\mathscr{P}(\mathfrak{R})$ possui um ordenamento parcial $\leq$ herdado de $\mathfrak{R}_{+}$, e dados quaisquer $E, F \in \mathscr{P}(\mathfrak{R})$, temos que existem o complemento $E^{\perp} \doteq \mathbb{1}-E$ de $E$, o ínfimo $E \wedge F \doteq s-\lim _{n \rightarrow \infty}(E F)^{n}$ (por $s-\lim$ entende-se o limite na topologia forte de $\mathfrak{R})$ e o supremo $E \vee F \doteq\left(E^{\perp} \wedge F^{\perp}\right)^{\perp}$ de $E$ e $F$ com respeito ao ordenamento $\leq{ }^{11}$ - ou seja, $\left(\mathscr{P}(\Re), \leq, \vee, \wedge,{ }^{\perp}\right)$ é um reticulado

\footnotetext{
${ }^{9}$ Note que todo $\mathscr{B}(\mathscr{H})$ é uma álgebra de von Neumann e, como tal, é o dual de um espaço de BANACH - este último, neste caso, é formado pelos seus elementos "trace class" [BR87]. A assertiva segue então do teorema de BANACH-AlaOgLu [RS80].

${ }^{10}$ Foi demonstrado por KADISON [Tak01] que uma $\mathrm{C}^{*}$-álgebra $\mathfrak{A}$ é uma $\mathrm{W}^{*}$-álgebra se e somente se toda rede crescente $\left\{A_{\alpha}\right\} \subset \mathfrak{A}_{+}$limitada superiormente possui supremo $\sup _{\alpha} A_{\alpha} \in \mathfrak{A}$ e os estados que satisfazem a condição (iii) do Teorema B.13 separam elementos em $\mathfrak{A}_{+}$, i.e., dado $0 \neq x \in \mathfrak{A}_{+}$, existe um estado normal $\omega$ tal que $\omega(A) \neq 0$.

${ }^{11}$ Dado um conjunto parcialmente ordenado $(S, \leq)$, o infimo de $x, y \in S$ é um elemento $x \wedge y \in S$ tal que $x \wedge y \leq x, y$ e $z \leq x, y \Rightarrow z \leq x \wedge y$, o supremo de $x, y \in S$ é um elemento $x \vee y \in S$ tal que $x, y \leq x \vee y$ e $x, y \leq w \Rightarrow x \vee y \leq w$, e uma operação de complemento é uma bijeção $\perp: S \rightarrow S$ que satisfaz $\left(x^{\perp}\right)^{\perp}=x,(x \wedge y)^{\perp}=x^{\perp} \vee y^{\perp}$ e $(x \vee y)^{\perp}=x^{\perp} \wedge y^{\perp}$. Se todo par $x, y \in S$ possui supremo e ínfimo, dizemos que $(S, \leq, \vee, \wedge)$ é um reticulado ("lattice") - em particular, todo reticulado é direcionado, i.e., dados $x, y \in S$, existe $z \geq x, y$. Se, mais ainda, $S$ possui uma operação de complemento ${ }^{\perp}$, então dizemos que $\left(S, \leq, \vee, \wedge,{ }^{\perp}\right)$ é um reticulado ortocomplementado.
} 
ortocomplementado.

Introduzimos a seguinte relação de equivalência em $\mathscr{P}(\mathfrak{R})$ : dados $E, F \in \mathscr{P}(\mathfrak{R})$, dizemos que $E \sim F$ se existe $W \in \mathfrak{R}$ tal que $W^{*} W=E$ e $W W^{*}=F$ (i.e., $W$ é uma isometria parcial com fonte $E$ e alvo $F$ ). Dizemos ainda que $E$ é uma subprojeção de $F$ se $E \leq F$ ( $E$ é própria se, além disso, $E \neq F$ ), e definimos a seguinte relação de ordem em $\mathscr{P}(\Re) / \sim \ni[E],[F]:[E] \neq[F]$ se $E$ é equivalente a uma subprojeção de $F$ (a definição claramente independe da escolha de representante). Usando uma *-representação fiel de $\mathfrak{R}$ num espaço de Hilbert $\mathscr{H}$, é fácil provar que $E \leq F$ se e somente se $E F=E$, justificando nossa terminologia. Mais ainda, usando a correspondência biunívoca $E \mapsto \operatorname{Ran} E$ que existe entre os elementos de $\mathscr{P}(\mathscr{B}(\mathscr{H}))$ e os subespaços fechados de $\mathscr{H}$, vemos que

$\operatorname{dim} \operatorname{Ran} E \leq+\infty$ se e somente se não existe $0 \neq F \lesseqgtr E$ tal que $F \sim E$.

Podemos definir abstratamente, então, que $E \in \mathscr{P}(\Re)$ é finita se satisfaz a condição acima, que obviamente não depende da realização de $\mathfrak{R}$ em $\mathscr{H}$. Dizemos ainda que $E$ é infinita, caso contrário.

DefiniçÃo B.5 (Murray-Von Neumann) Seja $\Re$ uma álgebra de von Neumann. Uma função $d: \mathscr{P}(\mathfrak{R}) \rightarrow \overline{\mathbb{R}}_{+} \cup\{+\infty\}$ é dita ser uma função dimensional se $d(E)=$ $0 \Rightarrow E=0, E \sim F \Rightarrow d(E)=d(F) e E \leq F^{\perp} \Rightarrow d(E \vee F)=d(E+F)=d(E)+d(F)$ - tais propriedades determinam d a menos de um múltiplo $\lambda \in \mathbb{R} \backslash\{0\}$, e $d(E)<+\infty$ se e somente se $E$ for finita. Dizemos que $\mathfrak{R}$ é finita se $d(\mathbb{1})<+\infty$, e infinita caso contrário. $\mathfrak{R}$ é semifinita se todo $E \in \mathscr{P}(\mathfrak{R})$ possui uma subprojeção finita $F \neq 0$, e puramente infinita se toda $E \in \mathscr{P}(\Re)$ é infinita.

Se $\mathfrak{R}$ é um fator, dizemos que $\mathfrak{R}$ é:

- Tipo $\mathrm{I}_{n}$ se $\operatorname{Ran} d=\lambda\{0,1, \ldots, n\}, n=0, \ldots,+\infty$ (equivalentemente, $\mathfrak{R}$ é tipo I se possui uma projeção minimal $E \neq 0$, i.e., $F \neq E \Rightarrow F=E$ ). $\Re$ é finito se e somente se $n<+\infty$;

- Tipo II se for semifinito, mas não for tipo I. $\mathfrak{R} e ́$, então, tipo $\mathrm{II}_{1}$ se $\mathrm{Rand}=\lambda[0,1]$ (equivalentemente, se $\mathfrak{R}$ for finito) e tipo $\mathrm{II}_{\infty}$ se $\operatorname{Ran} d=\overline{\mathbb{R}}_{+} \cup\{+\infty\}$;

- Tipo III se for puramente infinito, i.e., $\operatorname{Ran} d=\{0,+\infty\}$.

Vários dos resultados mais avançados acerca de álgebras de vON NEUmann fazem uso do conceito de peso, que generaliza o conceito de funcional linear positivo. Dada uma $W^{*}$-álgebra $\mathfrak{R}$, um peso em $\mathfrak{R}$ é uma função $\omega: \mathfrak{R}_{+} \rightarrow \overline{\mathbb{R}}_{+} \cup\{+\infty\}$ (onde convencionamos $0 .+\infty=0$ ) que satisfaz (i) $\omega(A)+\omega(B)=\omega(A+B)$ e (ii) $\omega(\alpha A)=\alpha \omega(A)$, 
para todo $A, B \in \mathfrak{R}_{+}, \alpha \in \overline{\mathbb{R}}_{+}$. Um peso $\tau$ é dito tracial (ou simplesmente um traço) se $\omega\left(A^{*} A\right)=\omega\left(A A^{*}\right)$, para todo $A$. Denominando $\mathfrak{R}_{\omega+} \doteq\left\{A \in \mathfrak{R}_{+}: \omega(A)<+\infty\right\}$ e $\mathfrak{L}_{\omega} \doteq\left\{A \in \mathfrak{R}: \omega\left(A^{*} A\right)<+\infty\right\}$, temos imediatamente que $\mathfrak{R}_{\omega+}$ é um cone hereditário em $\mathfrak{R}_{+}$, i.e., $0 \leq A \leq B \in \mathfrak{R}_{\omega+} \Rightarrow A \in \mathfrak{R}_{\omega+}$, e $\mathfrak{R}_{\omega} \doteq \operatorname{span}_{\mathbb{C}} \mathfrak{R}_{\omega+}=\mathfrak{L}_{\omega}^{*} \mathfrak{L}_{\omega}$. Assim, o peso $\omega$ se estende por linearidade a um funcional linear positivo na $*_{\text {-álgebra }} \mathfrak{R}_{\omega}$ - em particular, se $\omega$ é um traço, então $\omega(A B)=\omega(B A)$ para todo $A, B \in \mathfrak{R}_{\omega}$. Dizemos que um peso $\omega$ é normal se satisfaz a condição (iii) do Teorema B.13, fiel se $\omega \uparrow_{\Re_{\omega}}$ for fiel, e semifinito se $\mathfrak{R}_{\omega}$ for $\sigma$-fracamente densa em $\mathfrak{R}$. É possível provar que toda álgebra de vON Neumann $\mathfrak{R}$ admite um peso normal, fiel e semifinito, e que $\mathfrak{R}$ é semifinita se e somente se admitir um traço $\tau$ normal, fiel e semifinito [Tak01, Tak03a] - a recíproca da última assertiva é imediata, pois se $E$ é uma projeção em $\mathfrak{R}_{\tau}$, então $E$ é necessariamente finita.

Álgebras de von Neumann $\mathfrak{R} \sigma$-finitas, i.e., tais que todo subconjunto $S \in \mathscr{P}(\mathscr{R})$ mutualmente ortogonal (i.e., que satisfaz $E \leq F^{\perp}$ para todo $E, F \in S$ ) é enumerável, podem ser decompostas $\mathfrak{R}$ numa "integral direta" de fatores, em termos do conjunto de projeções centrais $\mathfrak{Z}(\mathfrak{R}) \cap \mathscr{P}(\mathscr{R})$. $\mathfrak{R}$ é $\sigma$-finita se e somente se admite um estado normal e fiel $\omega$ [BR87]. Assim, pode-se dizer que, para tais álgebras, a tarefa de classificação se reduz à classificação de fatores. Um refinamento da classificação dada pela Definição B.5, para fatores de tipo III, será apresentado sucintamente no final da Seção B.2.3.

\section{B.2.3 Teoria modular de ToMITA-TAKESAKI}

Estamos agora em condições de discutir uma estrutura típica de álgebras de vON Neumann, extremamente útil e poderosa. Considere uma $\mathrm{C}^{*}$-álgebra $\mathfrak{A}$ e um estado fiel $\omega$. Identificamos então a $W^{*}$-álgebra envolvente $\mathfrak{A}^{* *}=\pi_{\omega}(\mathfrak{A})^{\prime \prime} \doteq \mathfrak{R}$ no espaço de Hilbert $\mathscr{H}_{\omega} \doteq \mathscr{H}$, o qual possui, por conseguinte, um vetor cíclico e separante $\Omega$. Definamos os operadores anti-lineares densamente definidos $S_{0}$ (já mencionado en passant na Seção B.1) e $F_{0}$ como

$$
D\left(S_{0}\right)=\mathfrak{R} \Omega, S_{0} A \Omega \doteq A^{*} \Omega ; D\left(F_{0}\right)=\mathfrak{R}^{\prime} \Omega, S_{0} A^{\prime} \Omega \doteq A^{\prime *} \Omega
$$

$\left(F_{0}\right.$ é densamente definido, pois um vetor $\Phi$ é cíclico para uma álgebra de VON NEUMANn $\mathfrak{S}$ se e somente se for separante para $\left.\mathfrak{S}^{\prime}\right)$. Segue que $S_{0} \subseteq F_{0}^{*}$ e $F_{0} \subseteq S_{0}^{*}$, e, portanto, tanto $S_{0}$ e $F_{0}$ são fecháveis. Pode-se ainda provar que [BR87]

$$
F_{0}^{*}=\bar{S}_{0} \doteq S \text { e } S_{0}^{*}=\bar{F}_{0} \doteq F .
$$

DefiniçÃo B.6 Sejam J o operador anti-unitário (i.e., $J$ é anti-linear, inversível e tal que $\langle J \Phi, J \Psi\rangle=\overline{\langle\Phi, \Psi\rangle}=\langle\Psi, \Phi\rangle$ para todo $\Phi, \Psi \in \mathscr{H})$ e $\Delta$ o operador positivo da decomposição polar $S=J \Delta^{\frac{1}{2}}$ de $S$. Dizemos que $\Delta$ é o operador modular, e $J$, a conjugação modular, associados a $\Re, \Omega$. 
O nome tem sua origem em Análise Harmônica: ao definirmos a involução da *_ álgebra de convolução das funções contínuas de suporte compacto sobre um grupo topológico localmente compacto, precisamos introduzir a derivada de RADON-NIKÓDYM da medida de HAAR invariante à direita em relação à medida de HAAR invariante à esquerda, que é denominada função modular (essa função é igual a 1 para grupos Abelianos ou compactos). Segue de (B.1) que

$$
\begin{aligned}
& \text { 2) } \Delta=F S, \Delta^{-1}=S F, F=J \Delta^{-\frac{1}{2}}, J=J^{*}\left(\text { e, portanto, } J^{2}=\mathbb{1}\right) \text { e } \Delta^{-\frac{1}{2}}=J \Delta^{\frac{1}{2}} J \\
& \text { O resultado mais importante envolvendo } J \text { e } \Delta \text { é o }
\end{aligned}
$$

TeOrema B.14 (Tomita-TAKesaki [BR87, Tak03a]) Seja $\mathfrak{R}$ uma álgebra de von Neumann em $\mathscr{H}$ com vetor cíclico e separante $\Omega$, e $\Delta$ e J respectivamente o operador modular e a conjugação modular associados a $(\Re, \Omega)$. Então,

$$
J \mathfrak{R} J=\mathfrak{R}^{\prime} \text { e } \Delta^{i t} \mathfrak{R} \Delta^{-i t}=\mathfrak{R}, \forall t \in \mathbb{R} .
$$

Em particular, $t \mapsto \Delta^{i t}$ define um grupo uniparamétrico fortemente contínuo de operadores unitários em $\mathscr{H}$, com gerador $\log \Delta$, definido pelo Teorema Espectral, que, pelo Teorema B.14, implementa um grupo $\sigma$-fracamente contínuo de automorfismos de $\mathfrak{R}$ e de $\mathfrak{R}^{\prime}$ (neste último, o sinal de $t$ é trocado), denominado grupo modular. Para $\mathfrak{R}=\pi_{\omega}(\mathfrak{A})^{\prime \prime}$, onde $\mathfrak{A}$ é uma $\mathrm{C}^{*}$-álgebra, e $\Omega$ o vetor GNS cíclico e separante associado a um estado $\omega$, escrevemos, então, para cada $x \in \mathfrak{A}$ :

$$
\begin{gathered}
j(x) \doteq \pi_{\omega}^{-1}\left(J \pi_{\omega}(x) J\right), \mathrm{e} \\
\sigma_{t}^{\omega}(x) \doteq \pi_{\omega}^{-1}\left(\Delta^{i t} \pi_{\omega}(x) \Delta^{-i t}\right) .
\end{gathered}
$$

Note que as fórmulas (B.3) e (B.4) assumem valores em $\mathfrak{A}^{* *}$, i.e., $\pi_{\omega}^{-1}$ denota, em ligeiro mas inócuo abuso de notação, a sua única extensão $\sigma$-fracamente contínua a $\pi_{\omega}(\mathfrak{A})^{\prime \prime}$. Uma fórmula da mais alta importância que segue da definição dos objetos modulares é:

$$
\begin{gathered}
\left\langle\Delta^{\frac{1}{2}} \pi_{\omega}(x) \Omega, \Delta^{\frac{1}{2}} \pi_{\omega}(y) \Omega\right\rangle=\left\langle J \pi_{\omega}\left(x^{*}\right) \Omega, J \pi_{\omega}\left(y^{*}\right) \Omega\right\rangle=\left\langle\pi_{\omega}\left(y^{*}\right) \Omega, \pi_{\omega}\left(x^{*}\right) \Omega\right\rangle \\
\Downarrow \\
\omega\left(\sigma_{i / 2}^{\omega}(x) \sigma_{-i / 2}^{\omega}(y)\right)=\omega(y x) .
\end{gathered}
$$

A teoria modular de TOMITA-TAKESAKi pode ser estendida a álgebras de VON Neumann arbitrárias se empregarmos um peso normal, fiel e semifinito ao invés de um 
estado normal e fiel. As demonstrações, contudo, são consideravelmente mais complicadas.

Por fim, há uma relação rígida entre os grupos modulares associados a dois estados (ou, mais em geral, pesos semifinitos) normais e fiéis $\phi, \omega$ sobre uma álgebra de VON Neumann $\mathfrak{R}$, dada pelo

Teorema B.15 (Connes) Existe uma familia uniparamétrica $t \mapsto \Gamma_{t} \doteq(D \phi: D \omega)_{t}$ de elementos unitários de $\mathfrak{R}$ que satisfaz $\sigma_{t}^{\phi}(A)=\Gamma_{t} \sigma_{t}^{\omega}(A) \Gamma_{t}^{*}$ e a relação de cociclo $\Gamma_{t+s}=\Gamma_{t} \sigma_{t}^{\omega}\left(\Gamma_{s}\right)$. Em particular, se $\phi=\omega\left(U . U^{*}\right)$, onde $U \in \mathfrak{R}$ é um elemento unitário, então necessariamente $\Gamma_{t}=U^{*} \sigma_{t}^{\omega}(U)$.

Prova. Ver o Teorema 2.7.16 de [BR87] e o Teorema 5.3.34 de [BR97].

A expressão $\left.S(\phi \mid \omega) \doteq \frac{d}{d t} \omega\left((D \phi: D \omega)_{t}\right)\right|_{t=+0}$ define também a entropia relativa de $\phi$ em relação a $\omega$ [Ara76, BR97, OP93]. Os cociclos definidos no Teorema B.15 são de importância fundamental na classificação de fatores de tipo III realizada por ConNeS [BR87, Tak03a]. Seja $\mathfrak{R}$ um fator $\sigma$-finito, e $\mathbb{R} \ni u \mapsto \alpha_{g}$ um grupo uniparamétrico $\sigma$-fracamente contínuo de ${ }^{*}$-automorfismos de $\mathfrak{R}$, de modo que faz sentido falar da integral $\sigma$-fraca

$$
L^{1}(\mathbb{R}) \ni f \mapsto \alpha_{f}(A) \doteq \int f(t) \alpha_{t}(A) d t, A \in \mathfrak{R} .
$$

Isto é, o funcional $\mathfrak{R}_{*} \ni \phi \mapsto \phi\left(\alpha_{f}(A)\right) \doteq \int f(t) \phi\left(\alpha_{t}(A)\right) d t$ define um elemento de $\left(\mathfrak{R}_{*}\right)^{*}=\mathfrak{R}$ para cada $A \in \mathfrak{R}, f \in L^{1}(\mathbb{R})$. Defina a subálgebra de ponto fixo $\mathfrak{R}^{\alpha} \doteq\{A \in$ $\left.\mathfrak{R}: \alpha_{t}(A)=A, \forall t \in \mathbb{R}\right\}$. O espectro de ARVESON de $\alpha$. é dado pela intersecção dos suportes das distribuições temperadas a valores em $\mathfrak{R} f \mapsto \alpha_{f}(A)$ para $A \in \mathfrak{R}$, ou, equivalentemente,

$$
\sigma(\alpha) \doteq \mathbb{R} \backslash \bigcup\left\{U \subset \mathbb{R} \text { aberto: } \alpha_{f}(A)=0, \forall A \in \Re, f \in L^{1}(\mathbb{R}), \operatorname{supp} f \subset U\right\},
$$

e o espectro de Connes é dado por

$$
\Gamma(\alpha) \doteq \bigcap_{E \in \mathscr{P}(\mathfrak{R}) \cap \Re^{\alpha}} \sigma\left(\left.\alpha\right|_{\mathfrak{R} E}\right)
$$

Consideremos agora o grupo modular $\alpha .=\sigma^{\omega}$ associado ao estado normal e fiel $\omega$. Como o espectro de um operador auto-adjunto é invariante pela ação adjunta de um operador unitário [RS80], segue do Teorema B.15 que $\Gamma(\Re) \doteq \Gamma\left(\sigma^{\omega}\right)$ independe de $\omega$. Pode-se ainda provar [BR87, Tak03a] que $\exp \Gamma(\Re)=S(\Re) \backslash\{0\}$ é um subgrupo multiplicativo

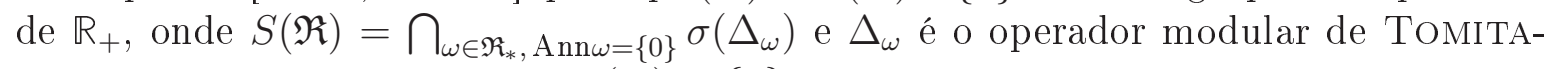
TAKESAKi associado a $\omega$, e que $\Gamma(\mathfrak{R})=\{0\}$ se e somente se $\mathfrak{R}$ for semifinito. Segue daí que as possibilidades restantes para $S(\Re)$ em virtude de sua estrutura multiplicativa 
- $S(\mathfrak{R})=\{0,1\}$

- $S(\mathfrak{R})=\{0\} \cup\left\{\lambda^{n}: n \in \mathbb{Z}\right\}, \lambda \in(0,1)$;

- $S(\mathfrak{R})=\overline{\mathbb{R}}_{+}$,

só se realizam para $\mathfrak{R}$ de tipo III.

Definição B.7 (Connes) Dizemos que $\mathfrak{R}$ é tipo $\operatorname{III}_{0}$ se $S(\mathfrak{R})=\{0,1\}$, tipo $\mathrm{III}_{\lambda}$, $\lambda \in(0,1)$, se $S(\mathfrak{R})=\{0\} \cup\left\{\lambda^{n}: n \in \mathbb{Z}\right\}, \lambda \in(0,1)$, e tipo $\mathrm{III}_{1}$ se $S(\mathfrak{R})=\overline{\mathbb{R}}_{+}$.

De particular interesse em Teoria Quântica de Campos são os fatores hiperfinitos (uma álgebra de VON NEUMANN $\mathfrak{R}$ é hiperfinita ou injetiva se for gerada pela união de uma seqüência de álgebras matriciais de dimensão crescente) de tipo $I I I_{1}$. Estes tipicamente aparecem como limites termodinâmicos de álgebras locais associadas a uma rede, tais como o fator de ARAKI-WOODS, dado pelo limite termodinâmico de álgebras de observáveis de sistemas de spin em temperatura finita [BR97]. Foi provado por HAAGERUP, completando resultados parciais anteriores de CONNES, que o fator de ArAKI-Woods é o único fator hiperfinito tipo III $_{1}$ a menos de *-isomorfismo [Tak03b]. Finalizamos esta Seção com dois critérios úteis para determinar se $\mathfrak{R}$ é tipo III.

TeOrema B.16 Dado um estado normal e fiel $\omega, \mathfrak{R}$ é tipo III se e somente se $\left.\sigma^{\omega}\right|_{\mathfrak{R} E}$ não for um grupo de automorfismos internos (i.e., não existe um grupo $\sigma$-fracamente continuo $\mathbb{R} \ni t \mapsto U_{t}$ de unitários de $\mathfrak{R}$ tal que $\sigma_{t}^{\omega}()=.U_{t}^{*} . U_{t}, \forall t \in \mathbb{R}$ ) para nenhum $E \in \mathscr{P}(\mathfrak{R}) \cap \mathfrak{Z}(\mathfrak{R})$.

Prova. Ver o Teorema 2.7.17 de [BR87] e a discussão precedendo o Teorema 5.3.35 de [BR97].

O segundo critério é devido a DRIESSLER [Dri77]:

Teorema B.17 ([Dri77]) Seja $\mathscr{H}$ um espaço de HilBerT separável, $\mathfrak{R}$ uma subálgebra de von Neumann de $\mathscr{B}(\mathscr{H})$ e $\Omega$ um vetor cíclico e separante para $\mathfrak{R}$. Suponha que existe uma subálgebra de VON NEUMANN infinita $\mathscr{R}_{1} \subset \mathfrak{R}$ e $\left\{\alpha_{n}\right\}_{n \in \mathbb{Z}_{+}}$uma seqüência de ${ }^{*}$-automorfismos de $\mathscr{B}(\mathscr{H})$ satisfazendo:

1. $\alpha_{n}\left(\Re_{1}\right) \subset \mathfrak{R}_{1}$, para todo $n$;

2. $w-\lim _{n \rightarrow \infty} \alpha_{n}\left(A_{1}\right)=\omega\left(A_{1}\right) \mathbb{1}$, para todo $A_{1} \in \mathfrak{R}_{1}, 0 \not \equiv \omega \in \mathfrak{R}_{1 *}$;

3. $s-\lim _{n \rightarrow \infty}\left[A, \alpha_{n}\left(A_{1}\right)\right]=\{0\}$, para todo $A \in \mathfrak{R}, A_{1} \in \mathfrak{R}_{1}$.

Então $\Re$ é tipo III. 
Prova. Suponha que $P \neq 0$ é uma projeção finita. Neste caso, $P \Re P$ é finita e, como vimos no final da Subseção B.2.2, possui um traço normal, fiel e semifinito $\tau$. Como $P \Omega$ é cíclico e separante para $P \Re P$ em $P \mathscr{H}$, segue do análogo do Teorema 2.5.31 em [BR87] para pesos [Tak03a] que $\tau$ é implementado por um único vetor tracial $\xi \in P \mathscr{H}$. Obtemos, então, que

$$
\lim _{n}\left\langle\xi, \alpha_{n}(A) P \alpha_{n}(B) \xi\right\rangle \stackrel{3 .)}{=} \lim _{n}\left\langle\xi, \alpha_{n}(A B) \xi\right\rangle \stackrel{2 .)}{=}\langle\xi, A B \xi\rangle=\langle\xi, B A \xi\rangle .
$$

O limite acima define, portanto, um traço normal, fiel e finito $\tau_{1}$ em $\mathfrak{M}_{1}$, i.e., $\tau_{1}(A)<+\infty$ para todo $A \in \mathfrak{R}_{1}$, implicando que $\mathfrak{R}_{1}$ é finita, o que é absurdo.

\section{B.2.4 Sistemas $\mathrm{C}^{*}$ - e $\mathrm{W}^{*}$-dinâmicos. Condição KMS}

Vamos chegar agora numa interpretação física para a fórmula (B.5). Primeiro, notar que, dado $A \in \mathfrak{R}$, as integrais $\sigma$-fracas

$$
\begin{gathered}
A_{n}=\sqrt{\frac{n}{\pi}} \int \sigma_{t}^{\omega}(A) e^{-n t^{2}} d t, n \in \mathbb{Z}_{+} \\
\left(\text {i.e., } \phi\left(A_{n}\right)=\sqrt{\frac{n}{\pi}} \int \phi\left(\sigma_{t}^{\omega}(A)\right) e^{-n t^{2}} d t \text { para todo } \phi \in \mathfrak{F}_{*}\right)
\end{gathered}
$$

definem unicamente uma seqüência de elementos $\left(A_{n}\right)$ que convergem $\sigma$-fracamente para $A$ (ver Proposição 2.5.18 de [BR87]), e tais que cada $A_{n}$ é um elemento analítico para $\sigma_{t}^{\omega}$, i.e., existe $\lambda>0$ e uma função $f_{A_{n}}: \mathbb{R}+i(-\lambda, \lambda) \rightarrow \mathfrak{R}$ tal que $f(t)=\sigma_{t}^{\omega}(A)$ para todo $t \in \mathbb{R}$ e $z \mapsto \phi(f(z))$ é analítica em $\mathbb{R}+i(-\lambda, \lambda)$ para todo $\phi \in \mathfrak{R}_{*}$. Logo, $\sigma_{t}^{\omega}$ possui um conjunto $\sigma$-fracamente denso de elementos analíticos $B$ em $\mathfrak{R}$. Para tais, escrevemos $f_{B}(z)=\sigma_{z}^{\omega}(B)$.

Retornemos a (B.5). Escolhendo $A=\mathbb{1}$ e $B$ um elemento analítico de $\mathfrak{R}$ na faixa $\mathbb{R}+i(-1 / 2,1 / 2)$ (isso sempre pode ser obtido por rescalonamento adequado de $f_{B}$ ), temos que a função $F(z)=\omega\left(\sigma_{z+i / 2}^{\omega}(B)\right)$ é contínua e limitada em $\mathbb{R}+i[-1,0]$, analítica em $\mathbb{R}+i(-1,0)$, e periódica de período $1 \mathrm{em} \Im z$, devido a (B.5). Portanto, $F$ admite uma extensão analítica limitada a todo $\mathbb{C}$ (inclusive em $\mathbb{R}+i \mathbb{Z}$, pelo princípio de reflexão de Schwarz). Portanto, pelo teorema de Liouville, $F$ deve ser constante, i.e., $F(z)=\omega(B)$ para todo $z \in \mathbb{C}$, em particular para $z=t \in \mathbb{R}$. Como os elementos analíticos são densos em $\mathfrak{R}$, segue que $\omega$ é invariante por $\sigma_{t}^{\omega}$.

Mais em geral, dados $A, B \in \mathfrak{R}$, podemos escolher seqüências $\left(A_{n}\right),\left(B_{n}\right)$ tais que $\left\|A_{n}\right\| \leq\|A\|,\left\|B_{n}\right\| \leq\|B\|$ e $A_{n}^{(*)} \Omega \rightarrow A^{(*)} \Omega, B_{n}^{(*)} \Omega \rightarrow B^{(*)} \Omega$ (isto é sempre possível pelo Teorema de Densidade de KAPLANSKy - ver [BR87], Teorema 2.4.16). Neste caso, segue de (B.5) e do Teorema de Três Linhas de Phragmén-Lindelöf (ver Teorema 
6.4 em [Lan99]) que $\omega\left(A_{n} f_{B_{n}}(z)\right)$ converge uniformemente em $\mathbb{R}+i[-1 / 2,1 / 2]$ para uma função $F_{A, B}^{\prime}$ contínua e limitada em $\mathbb{R}+i[-1 / 2,1 / 2]$ e analítica em $\mathbb{R}+i(-1 / 2,1 / 2)$ (ver Proposição 5.3.7 em [BR97] para uma demonstração mais detalhada), tal que, para todo $t \in \mathbb{R}$,

$$
F_{A, B}^{\prime}(t+i / 2)=\omega\left(A \sigma_{t}^{\omega}(B)\right) \text { e } F_{A, B}^{\prime}(t-i / 2)=\omega\left(\sigma_{t}^{\omega}(B) A\right) .
$$

Podemos reformular a assertiva acima da seguinte forma: seja $f \in \mathscr{S}(\mathbb{R})$ tal que $\hat{f} \in \mathscr{C}_{c}^{\infty}(\mathbb{R})$. Neste caso, existe $R>0$ tal que supp $\hat{f} \subset[-R, R]$ e, portanto, pelo Teorema de PALEY-WieneR [Hör90], $f$ é uma função inteira que satisfaz $|f(x+i y)|=$ $O\left(x^{-\infty} e^{R|y|}\right.$ ) para todo $x, y \in \mathbb{R}$ (de fato, esse Teorema nos diz também que essa estimativa caracteriza completamente as $f$ 's que satisfazem a hipótese acima). Podemos então reescrever (B.7) como

$$
\int f(t+i / 2) \omega\left(A \sigma_{t}^{\omega}(B)\right) d t=\int f(t-i / 2) \omega\left(\sigma_{t}^{\omega}(B) A\right) d t, \forall f: \hat{f} \in \mathscr{C}_{c}^{\infty}(\mathbb{R}) .
$$

DEFinição B.8 Sejam $\mathfrak{A}$ uma $C^{*}$-álgebra $e \mathbb{R} \ni t \mapsto \alpha_{t}$ um grupo uniparamétrico de *automorfismos de $\mathfrak{A}$, que assumimos por simplicidade ser fortemente contínuo, i.e., $\left\|\alpha_{t}(x)-x\right\| \stackrel{t \rightarrow 0}{\longrightarrow} 0$ para todo $x \in \mathfrak{A}$. O par $\left(\mathfrak{A}, \alpha_{t}\right)$ é dito ser um sistema $\mathrm{C}^{*}$-dinâmico. Se $\mathfrak{A}$ é uma álgebra de VON NEUMANN e $\alpha$ é $\sigma$-fracamente contínuo, dizemos que o $\operatorname{par}\left(\mathfrak{A}, \alpha_{t}\right)$ é um sistema $\mathrm{W}^{*}$-dinâmico.

Note que, se $\mathfrak{A}$ é uma $\mathrm{C}^{*}$-subálgebra em $\mathscr{B}(\mathscr{H})$ par algum $\mathscr{H}$, o sistema $\mathrm{C}^{*}$ dinâmico $\left(\mathfrak{A}, \alpha_{t}\right)$ se estende unicamente ao sistema $W^{*}$-dinâmico $\left(\mathfrak{A}^{\prime \prime}, \alpha_{t}\right)$, devido ao Teorema B.11.

Definição B.9 Seja $\left(\mathfrak{A}, \alpha_{t}\right)$ um sistema $C^{*}$ (resp. $W^{*}$-)dinâmico, $\beta \in \mathbb{R} \cup\{ \pm \infty\}$. Um estado (resp. normal) $\omega$ sobre $\mathfrak{A}$ é dito um estado $(\alpha, \beta)$-KMS se satisfaz a condição de Kubo, Martin e Schwinger: dados $x, y \in \mathfrak{A}$, existe uma função $F_{x, y}$ contínua $e$ limitada em $\mathbb{R}+(\operatorname{sgn} \beta) i[0,|\beta|]$ e analitica em $\mathbb{R}+(\operatorname{sgn} \beta) i(0,|\beta|)$, tal que

$$
F_{x, y}(t)=\omega\left(x \alpha_{t}(y)\right) \text { e } F_{x, y}(t+i \beta)=\omega\left(\alpha_{t}(y) x\right) .
$$

No caso $\beta=0, \omega$ satisfaz $\omega(x y)=\omega(y x)$ para todo $x, y$; dizemos então que $\omega$ é um estado tracial. No caso $\beta=+\infty$, dizemos que $\omega$ é um estado fundamental.

Segue de considerações similares às feitas para o grupo modular que um estado $(\alpha, \beta)$-KMS $\omega$ é invariante por $\alpha_{t}$, e, pela unicidade da representação GNS, existe uma única implementação de $\alpha_{t}$ por operadores unitários $U_{\alpha}(t)$ em $\mathscr{H}_{\omega}$. Uma conseqüência disto é que a (única) extensão normal de um estado $(\alpha, \beta)$-KMS em $\mathfrak{A}$ para $\mathfrak{A}^{* *}$ é também um estado $(\alpha, \beta)$-KMS para o sistema $\mathrm{W}^{*}$-dinâmico $\left(\mathfrak{A}^{* *}, \alpha_{t}\right)$, onde $\alpha_{t}$ denota a 
sua extensão $\sigma$-fracamente contínua.

A importância desse conceito se deve ao fato de que a condição KMS caracteriza o equilíbrio termodinâmico do estado $\omega$ na temperatura $T=\left(k_{B} \beta\right)^{-1}$, onde $k_{B}$ é a constante de Boltzmann. De fato, em sistemas quânticos com um número finito de graus de liberdade, a condição KMS caracteriza os estados de GIBBS $\omega_{\beta}=\frac{\operatorname{Tr}\left(e^{-\beta H} \text {. }\right)}{\operatorname{Tr}\left(e^{-\beta H}\right)}$, onde $H$ é o operador Hamiltoniano que gera $\alpha_{t}$. A condição KMS, no entanto, sobrevive ao limite termodinâmico, o que não ocorre com a caracterização de equilíbrio baseada em estados de GibBs. Sua formulação original, dada por Kubo, Martin e Schwinger, era baseada em funções de GREEN associadas a operadores de criação e aniquilação, tendo sido refraseada para sistemas $\mathrm{C}^{*}$-dinâmicos na versão dada pela Definição B.9 por HaAg, Hugenholtz e Winnink [HHW67].

Equivalentemente, pelas considerações acima, $\omega$ é um estado $(\alpha, \beta)$-KMS se e somente se, para toda $f$ tal que $\hat{f} \in \mathscr{C}_{c}^{\infty}(\mathbb{R})$,

$$
\int f(t) \omega\left(x \alpha_{t}(y)\right) d t=\int f(t+i \beta) \omega\left(\alpha_{t}(y) x\right) d t, \forall x, y \in \mathfrak{A} .
$$

O caso $\beta=+\infty$ é iluminado pela seguinte consideração: o primeiro membro de (B.9) nada mais é do que a transformada de FouRIER distribucional de $\omega\left(x \alpha_{t}(y)\right)$. Se $\operatorname{supp} \hat{f} \subset \mathbb{R}_{-}$, segue que $f(t+i \beta)$ decai exponencialmente à medida que $\beta \rightarrow+\infty$, e, portanto, o segundo membro de (B.9) tende a zero nesse limite. Assim, concluímos que o suporte da transformada de FoURIER distribucional de $\pi_{\omega}\left(\alpha_{t}(x)\right) \Omega$ está contido em $\overline{\mathbb{R}}_{+}$, justificando o nome "estado fundamental" para $\omega$.

Para $0<\beta<+\infty$, um estado $(\alpha, \beta)$-KMS $\omega$ satisfaz a seguinte "relação de flutuação-dissipação": $\omega\left(x^{*} \alpha_{t}(x)\right)$ e $\omega\left(\alpha_{t}(x) x^{*}\right)$ são distribuições positivas; pelo Teorema de BOCHNER-SCHWARTZ [RS75], as transformadas de FOURIER distribucionais

$$
\hat{\mu}_{x}(\hat{f}) \doteq \int f(t) \omega\left(x^{*} \alpha_{t}(x)\right) d t, \hat{\nu}_{x}(\hat{f}) \doteq \int f(t) \omega\left(\alpha_{t}(x) x^{*}\right) d t
$$

definem medidas de RADON em $\mathbb{R}$. Uma maneira de ver isso é usando a decomposição espectral dos operadores unitários $U_{\alpha}(t)$ que implementam $\alpha_{t}$. Podemos, então, ver que $\hat{\mu}_{x}$ e $\hat{\nu}_{x}$ são absolutamente contínuas uma em relação à outra, e a derivada de RADON-NIKÓDYM de $\hat{\mu}_{x}$ em relação a $\hat{\nu}_{x}$ é dada por

$$
\frac{d \hat{\mu}_{x}}{d \hat{\nu}_{x}}(p)=e^{-\beta p}, \text { ou, formalmente, } d \hat{\mu}_{x}(p)=e^{-\beta p} d \hat{\nu}_{x}(p)
$$

Se considerarmos as medidas com sinal $\hat{\delta}_{x, y}$ e $\hat{\phi}_{x, y}$ dadas respectivamente pelas transformadas de Fourier de $\omega\left(\left[x, \alpha_{t}(y)\right]\right)$ e $\omega\left(\left\{x, \alpha_{t}(y)\right\}\right)(\{a, b\} \doteq a b+b a$ denota o anti- 
comutador de $a, b \in \mathfrak{A})$, temos que

$$
d \hat{\delta}_{x, y}(p)=\tanh \left(\frac{\beta p}{2}\right) d \hat{\phi}_{x, y}(p)
$$

que é precisamente a relação de flutuação-dissipação que caracteriza a condição KMS, relacionando perturbações limitadas da dinâmica com flutuações das medidas de observáveis ao longo do tempo. Outras relações equivalentes a (B.10) e (B.11) podem ser analogamente obtidas entre outras funções de GREEN associadas a $\omega\left(x^{*} \alpha_{t}(y)\right)$.

Finalmente, vemos que todo estado normal e fiel $\omega$ sobre uma álgebra de VON Neumann $\mathfrak{R}$ é um estado $\left(\sigma_{-\beta t}^{\omega}, \beta\right)$-KMS. ${ }^{12}$ Este exemplo, num certo sentido, é o caso mais geral possível: conversamente, dado um estado $(\alpha, \beta)$-KMS $\omega$ sobre uma $\mathrm{C}^{*}$-álgebra $\mathfrak{A}$, segue que

LEMA B.18 $\Omega$ é separante para $\pi_{\omega}(\mathfrak{A})^{\prime \prime}$.

Prova. Primeiro, notar que, se $\mathfrak{A}$ é uma $\mathrm{C}^{*}$-subálgebra de $\mathscr{B}(\mathscr{H})$ para algum espaço de Hilbert $\mathscr{H}$ e $\omega=\langle\Omega, \Omega\rangle$ é um estado sobre $\mathfrak{A}$ tal que $\Omega$ é cíclico para $\mathfrak{A}$ e Ann $\omega$ é *-invariante, então $\Omega$ é separante para $\mathfrak{A}$ : dado $x$ tal que $\omega\left(x^{*} x\right)=0$, temos que $y x \Omega=0$ para todo $y \in \mathfrak{A}$. Mas, neste caso, $(y x)^{*} \Omega=x^{*} y^{*} \Omega=0$. Pela ciclicidade de $\Omega$, segue que $x=0$. Retornando a nossas hipóteses, considere agora a $\mathrm{C}^{*}$-subálgebra $\mathfrak{R}=\pi_{\omega}(\mathfrak{A})^{\prime \prime}$ de $\mathscr{B}\left(\mathscr{H}_{\omega}\right)$. Obviamente, $\Omega$ é cíclico para $\mathfrak{R}$. Tomando $A \in \mathfrak{R}$ tal que $\omega\left(A^{*} A\right)=0$, considere a função $F_{A^{*}, A}(z)$ como na Definição B.9. Então, $F_{A^{*}, A}(t)=\omega\left(A^{*} \alpha_{t}(A)\right)=\left\langle A \Omega, U_{\alpha}(t) A \Omega\right\rangle=0$. Pelo Teorema "Edge-of-the-Wedge" [SW00], segue que $F_{A^{*}, A}(z)=0$ para $z \in \mathbb{R}+i[0, \beta]$. Mas, neste caso, $F_{A^{*}, A}(i \beta)=\omega\left(A A^{*}\right)=0$. Portanto, pelo argumento acima, $A=0$.

Considere agora a seguinte situação: dado um sistema $W^{*}$-dinâmico $\left(\Re, \alpha_{t}\right)$ e um estado normal $(\alpha, \beta)$-KMS, segue do Lema B.18 que $\omega$ é fiel em $\pi_{\omega}(\Re)$. Entretanto, Ann $\omega$ é então um ideal $\sigma$-fracamente fechado à esquerda e à direita. Invocando a Proposição B.10, existe uma projeção $E \in \mathfrak{R} \cap \mathfrak{R}^{\prime}$ tal que Ann $\omega=(\mathbb{1}-E) \mathfrak{R}$. Assim, $\omega(\mathbb{1}-E)=\{0\}$ e $\omega$ é fiel em $\mathfrak{R} E$. Mais ainda,

$$
\omega(A E)=\omega(A)=\left\langle\Omega, \pi_{\omega}(A) \Omega\right\rangle
$$

Concluímos esta Subseção com o seguinte surpreendente resultado devido a TAKESAKI, que segue da teoria modular apresentada na Subseção B.2.3:

\footnotetext{
${ }^{12}$ Curiosamente, o trabalho de HaAg, Hugenholtz e Winnink [HHW67] precedeu por pouco o trabalho original não publicado de Tomita [Tom67], e, de certo modo, motivou a incepção da teoria modular, conforme pode ser visto pela exposição pioneira de TAKESAKI [Tak70].
} 
Teorema B.19 (Takesaki [BR97, Tak03a]) Sejam $\Re$ uma álgebra de von Neumann e $\omega$ um estado normal sobre $\mathfrak{R}$. Então, são equivalentes:

1. $\omega$ é fiel em $\pi_{\omega}(\mathfrak{R})$, i.e., existe uma projeção $E \in \mathfrak{R} \cap \mathfrak{R}^{\prime}$ tal que $\omega(\mathbb{1}-E)=0$ e $\omega\rceil_{\Re E}$ é fiel.

2. Existe um grupo $\sigma$-fracamente continuo $\alpha_{t}$ de *automorfismos de $\Re$ tal que $\omega$ é um estado KMS para o sistema $W^{*}$-dinâmico $\left(\Re, \alpha_{t}\right)$.

Mais ainda, se as condições acima são satisfeitas, segue que $\alpha_{t}(E)=E$, e $\alpha_{t}\lceil\Re E$ coincide com o grupo modular de $\mathfrak{R}$ E associado a $\omega$. Em particular, $\left.\alpha_{t}\right|_{\mathfrak{R} E}$ é unicamente determinado por $\omega$.

Prova. $\quad$ Ver o Teorema 5.3.10 de [BR97].

\section{B.3 *-álgebras localmente convexas e álgebras de BORCHERS-UHLMANN}

Lembramos, seguindo [Rud91] e [RS80], que um espaço vetorial topológico (sempre aqui assumido HAUSDORFF) $X$ (sempre sobre $\mathbb{C}$, a menos que seja dito o contrário) é localmente convexo se admite um sistema fundamental de vizinhanças abertas $\mathscr{V}=\left\{V_{\alpha} \ni 0\right\}_{\alpha \in I}$ tal que cada uma dessas vizinhanças é convexa, i.e., $t x+(1-t) y \in V_{\alpha}$ para todo $x, y \in V_{\alpha}, t \in(0,1)$. Equivalentemente, um espaço vetorial topológico é localmente convexo se admite uma família $\left\{\|\cdot\|_{\beta}\right\}_{\beta \in J}$ de seminormas (i.e., aplicações $\|.\|_{\alpha}: X \rightarrow \bar{R}_{+}$que satisfazem as mesmas propriedades de uma norma, exceto possivelmente $\|x\|=0 \Rightarrow x=0$ ) que separa pontos em $X$, i.e., dados $x \neq y \in X$, existe $\beta \in J$ tal que $\|x\|_{\beta} \neq\|y\|_{\beta}$. Um sistema de vizinhanças abertas localmente convexas da origem correspondente a esta família de seminormas é dado, por exemplo, por $V_{\beta_{1}, \ldots, \beta_{k}, n}=\left\{x \in X:\|x\|_{\beta_{i}}<n_{i}^{-1}, \forall i=1, \ldots, k, n_{i} \in \mathbb{Z}_{+}\right\}$. Conversamente, notar que todo espaço vetorial localmente convexo admite um sistema fundamental de vizinhanças abertas, convexas e balanceadas (i.e., $e^{i t} V_{\alpha}=V_{\alpha}$, para todo $t \in[0,2 \pi)$ ). Definindo $\|x\|_{\beta} \doteq \inf \left\{t>0: t^{-1} x \in V_{\beta}\right\}$, segue da continuidade da multiplicação por escalares e do fato de que $V_{\beta}$ é balanceada que $\|.\|_{\beta}$ define uma seminorma contínua - note que $\left\|V_{\beta}\right\|_{\beta} \subset[0,1)$, pois $V_{\beta}$ é aberto. Mais ainda, a família $\left\{\|\cdot\|_{\beta}\right\}_{\beta \in J}$ de seminormas separa pontos em $X$, pois para todo $x \neq 0$ existe $\beta$ tal que $x \notin V_{\beta}$ e, em particular, $\|x\|_{\beta} \geq 1$.

Nosso exemplo representativo de espaço vetorial topológico localmente convexo será $\mathscr{D}(\mathscr{O})=\mathscr{C}_{c}^{\infty}(\mathscr{O}), \mathscr{O} \subset R^{d}$ aberto, cuja topologia localmente convexa é definida da seguinte forma: esse espaço é dado pela união de subespaços

$$
\bigcup_{n=1}^{\infty} \mathscr{D}_{K_{n}}(\mathscr{O}),
$$


onde $\mathscr{D}_{K_{n}}(\mathscr{O}) \doteq\left\{f \in \mathscr{D}(\mathscr{O}): \operatorname{supp} f \subset K_{n}\right\}$ possui topologia localmente convexa dada pelas seminormas

$$
\|f\|_{k, K_{n}}=\sum_{|\alpha| \leq k} \sup _{K_{n}}\left|\partial^{(\alpha)} f\right|
$$

e $K_{n}=\bar{K}_{n} \Subset \mathscr{O}$ satisfaz $K_{n} \Subset K_{n+1}$ e $\cup_{n} K_{n}=\mathscr{O}$ (i.e., a coleção $\left\{K_{n}\right\}_{n \in \mathbb{Z}_{+}}$é uma exaustão de $\mathscr{O})$, implicando $\mathscr{D}_{K_{n}}(\mathscr{O}) \subseteq \mathscr{D}_{K_{n+1}}(\mathscr{O})$ de maneira que a topologia de $\mathscr{D}_{K_{n}}(\mathscr{O})$ é claramente induzida pela topologia de $\mathscr{D}_{K_{n+1}}(\mathscr{O})$. A topologia de $\mathscr{D}(\mathscr{O})$ é a topologia mais fina tal que as inclusões $\mathscr{D}_{K_{n}}(\mathscr{O}) \hookrightarrow \mathscr{D}(\mathscr{O})$ são contínuas para todo $n$, denominada limite indutivo $\mathrm{d}\left(\right.$ as topologias d)a coleção $\left(\mathscr{D}_{K_{n}}(\mathscr{O})\right)_{n \in \mathbb{Z}_{+}}$.

Uma coleção separante de seminormas que defina a topologia de um limite indutivo não é (note bem!) necessariamente enumerável, embora a coleção de seminormas dada acima o seja (i.e., nem sempre estas são suficientes para gerar a topologia de um limite indutivo). Se, no entanto, $\mathscr{O}$ é substituído por $K$ compacto e/ou os requerimentos de suporte são abandonados, e enumerarmos a família de seminormas dada acima como $\|\cdot\|_{k}, k=1,2, \ldots$ Definindo

$$
d(x, y) \doteq \sum_{k=1}^{\infty} \frac{\|x-y\|_{k}}{2^{k}\left(1+\|x-y\|_{k}\right)},
$$

temos que $d(.,$.$) define uma métrica invariante por translações que gera a topologia$ do espaço. Temos, não obstante, que $\mathscr{C}_{(c)}^{\infty}(\mathscr{O})$ e $\mathscr{C}_{(c)}^{\infty}(K)$ são seqüencialmente completos, i.e., toda seqüência de CAUCHY (para espaços topológicos em geral, uma seqüência $\left(x_{n}\right)$ é dita de CAuchy se, para toda vizinhança $V$ de 0 , existe $N \in \mathbb{Z}_{+}$tal que $x_{n}-x_{m} \in V$ para todo $n, m>N)$ converge. Em particular, $\mathscr{C}^{\infty}(\mathscr{O})$ e $\mathscr{C}_{(c)}^{\infty}(K)$ são espaços métricos completos (espaços de FRÉCHET). A topologia dos respectivos duais topológicos de distribuições é equivalente à topologia de convergência pontual de seqüências, sendo que tal topologia também é seqüencialmente completa.

Podemos agora dar a

DefiniçÃo B.10 Uma *-álgebra localmente convexa é uma *álgebra $\mathfrak{F}$ que, como espaço vetorial, é localmente convexa, e tal que as operações de produto e involução são continuas nesta topologia. Exigimos que as seminormas $\|\cdot\|_{\alpha}$ de $\mathfrak{F}$ satisfaçam a propriedade $C^{*}$, i.e., $\left\|x^{*} x\right\|_{\alpha}=\|x\|_{\alpha}^{2}$, para todo $\alpha$.

Vamos agora construir o exemplo que mais nos interessa, a álgebra de BorchersUhlmann [Bor62, Uhl62, Bor65]. O espaço $\mathscr{D}(\mathscr{O})$ é nuclear: existe uma única topologia localmente convexa no produto tensorial algébrico $\mathscr{D}(\mathscr{O}) \otimes \mathscr{D}(\mathscr{O}) \subset \mathscr{D}(\mathscr{O} \times \mathscr{O})$ que estende a topologia de $\mathscr{D}(\mathscr{O})$. O completamento de CAUCHY de $\mathscr{D}(\mathscr{O}) \otimes \mathscr{D}(\mathscr{O})$ nesta topologia é precisamente $\mathscr{D}(\mathscr{O} \times \mathscr{O})$. Dualmente, o Teorema do Núcleo de L. 
SCHWARTz [Hör90] asserta que a toda aplicação linear contínua $K: \mathscr{D}(\mathscr{O}) \rightarrow \mathscr{D}^{\prime}(\mathscr{O})$ corresponde um único $u \in \mathscr{D}^{\prime}(\mathscr{O} \times \mathscr{O})$ tal que $(K \phi)(\psi)=u(\phi \otimes \psi)$.

DefiniçÃo B.11 A álgebra de BorChers-Uhlmann associada a $\mathscr{O}$ é a *álgebra unital localmente convexa $\mathfrak{F}(\mathscr{O})$ dada por

$$
\mathfrak{F}(\mathscr{O}) \doteq \sum_{k=0}^{\infty} \otimes^{k} \mathscr{C}_{c}^{\infty}(\mathscr{O}),
$$

onde $\otimes^{0} \mathscr{C}_{c}^{\infty}(\mathscr{O})=\mathbb{C} \mathbb{1}$ denota a adjunção da identidade $\mathbb{1}$. Mais precisamente, é a álgebra $\overline{\mathbb{Z}}_{+}$-graduada composta de elementos $f=\left(f_{(0)}, f_{(1)}, \ldots\right)$, onde $f_{(k)} \in \otimes^{k} \mathscr{C}_{c}^{\infty}(\mathscr{O})$ e $f_{(k)}=0$ exceto para um conjunto finito de valores de $k$. As operações algébricas são dadas por:

- $\alpha f+\beta g=\left(\alpha f_{(0)}+\beta g_{(0)}, \alpha f_{(1)}+\beta g_{(1)}, \ldots,\right)$;

- $f g=f \otimes g=\left(f_{(0)} g_{(0)}, f_{(1)} g_{(0)}+f_{(0)} g_{(1)}, \ldots, \sum_{i+j=k} f_{(i)} \otimes g_{(j)}, \ldots\right)$;

- $f^{*}=\left(f_{(0)}^{*}, f_{(1)}^{*}, \ldots\right)$, onde $f_{(0)}^{*}=\overline{f_{(0)}}$ e, para $f_{(k)}=f_{(k)}^{1}\left(x_{1}\right) \cdots f_{(k)}^{k}\left(x_{k}\right), x_{i} \in \mathscr{O}, i=$ $1, \ldots, k$, definimos $f_{(k)}^{*}=\overline{f_{(k)}^{k}\left(x_{1}\right)} \cdots \overline{f_{(k)}^{1}\left(x_{k}\right)}$, estendendo a operação a $\otimes^{k} \mathscr{C}_{c}^{\infty}(\mathscr{O})$ por linearidade.

As seminormas de $\mathfrak{F}(\mathscr{O})$ são as herdadas de $\mathscr{C}_{c}^{\infty}(\mathscr{O})$. Segue imediatamente da regra de LEIBNIZ que estas seminormas satisfazem a propriedade $C^{*}$.

Um estado $\omega$ sobre $\mathfrak{F}(\mathscr{O})$ é dado por uma hierarquia de distribuições $\omega_{n} \in \mathscr{D}^{\prime}\left(\mathscr{O}^{n}\right)$, $\omega_{0}\left(f_{(0)} \mathbb{1}\right)=f_{(0)}$, sujeitas ao requerimento de positividade: $\sum_{n, m=0}^{k} \omega_{n+m}\left(f_{(n)}^{*} \otimes f_{(m)}\right) \geq 0$, $\forall f_{(n)} \in \otimes^{n} \mathscr{C}_{c}^{\infty}(\mathscr{O}), n=0, \ldots, k, k \in \overline{\mathbb{Z}}_{+}$. Note que a positividade é suficiente para garantir a continuidade fraca de $\omega_{n}$, e, de fato, uma boa dose de regularidade é garantida a priori para $\omega_{n}$. Demanda-se diretamente que as $\omega_{n}$ 's definem distribuições para incluir a possibilidade de $\omega$ não ser um funcional positivo (uma situação em que isso ocorre é o caso de campos de calibre).

A representação WightmAN-GNS de $\mathfrak{F}(\mathscr{O})$ associada a $\omega$ produz uma distribuição $\phi$ em $\mathscr{C}_{c}^{\infty}(\mathscr{O})$ que assume valores nos operadores fechados e (densamente) definidos em $\mathfrak{F}(\mathscr{O}) /$ Ann $\omega \subset \mathscr{H}_{\omega} . \phi$ satisfaz a condição de hermiticidade $\phi(\bar{f})^{*}=\phi(f)$.

Tudo o que fizemos acima passa sem mudanças essenciais de $\mathbb{R}^{d}$ para uma variedade $d$-dimensional $\mathscr{M}$ qualquer. Mais ainda, esse exemplo é a base para construirmos a álgebra de Borchers-UHLmANn associada a campos de spin arbitrário, o que será 
feito esboçadamente a seguir. Considere um fibrado vetorial $\mathscr{E} \stackrel{p}{\longrightarrow} \mathscr{M}$ com fibra $E \cong \mathbb{R}^{D}$. Dado $\mathscr{O} \subset \mathscr{M}$ aberto, tomamos

$$
\mathfrak{F}(\mathscr{O}) \doteq \sum_{k=0}^{\infty} \otimes^{k} \Gamma_{c}^{\infty}(\mathscr{O}, \mathscr{E}) .
$$

Neste caso, a função de $n$ pontos $\omega_{n}$ associada a um estado $\omega$ assume valores em $\otimes^{n} E$, e o campo obtido é uma $D$-tupla de distribuições a valores em operadores.

Chamamos aqui a atenção para o fato, extremamente importante, de que a álgebra de BorChers-Uhlmann contém apenas informação cinemática sobre a teoria, determinando o suporte, o caráter tensorial e as simetrias internas globais do campo ou multipleto de campos. Toda a informação dinâmica da teoria (equações de movimento, relações de comutação, espectro, etc.) está contida em $\omega$. Podemos alternativamente definir $\phi$ tomando o quociente de $\mathfrak{F}(\mathscr{O})$ por algum *-ideal $\mathfrak{I}$ (tratamos várias instâncias deste cenário nos Capítulos 3 e 4). Neste caso, um estado bona fide $\omega$ sobre o campo $\phi$ deve satisfazer Ann $\omega \supset \mathfrak{I}$, pois segue do Terceiro Teorema de *-Isomorfismo (Teorema B.4), suplementado pela Observação B.2, que $(\mathfrak{F}(\mathscr{O}) / \mathfrak{I}) /(\operatorname{Ann} \omega / \mathfrak{I}) \cong \mathfrak{F}(\mathscr{O}) / \operatorname{Ann} \omega$.

Pode-se construir, sob circunstâncias especiais (como, por exemplo, no caso de uma teoria quântica de campos satisfazendo os axiomas de WiGHTMAN), uma versão da teoria modular de TOMITA-TAKESAKI para *-álgebras como a álgebra de BORCHERSUhlmann. Este problema, tratado pela primeira vez por Bisognano e Wichmann [BW75, BW76], é atacado de maneira sistemática em [Ino98]. 



\section{APÊNDICE C}

\section{Categorias e funtores}

Aqui, introduzimos de maneira minimalista os conceitos necessários da teoria de categorias e funtores, uma linguagem que surgiu com a finalidade de organizar as estruturas que aparecem nas diferentes áreas da Matemática. Nossa referência para este Apêndice é o texto clássico [Lan98] de MAC LANE, um dos pais do conceito.

Cabe aqui um breve comentário sobre o uso da teoria de conjuntos feito quando se fala em categorias. Estritamente falando, o conceito de categoria ${ }^{1}$ (Definições C.1 e C.2) não faz menção essencial a nenhum dos axiomas da teoria dos conjuntos. De fato, é comum (e bastante útil) considerar categorias "grandes", i.e., em que os objetos e as setas (morfismos) constituem agregados mais gerais que conjuntos, tais como classes ${ }^{2}$. Segundo o sistema de axiomas conjuntísticos de GÖDEL e BERnAYs, a noção de classe é realizada por todas as instâncias positivas de uma determinada função proposicional que define as propriedades dos objetos da classe. Para nossas necessidades, bastarnos-á dizer que uma classe é um conjunto se cada uma das instâncias positivas que a formam se reduz a um único elemento do universo. Em suma, conjuntos são agregados "pequenos" (i.e., elementos do universo) e classes, agregados "grandes". ${ }^{3}$ Não teremos necessidade de conceitos conjuntísticos mais gerais.

\section{C.1 Rudimentos}

DEFINIÇÃO C.1 Uma metacategoria consiste de:

\footnotetext{
${ }^{1}$ Mais precisamente, de metacategoria - ver Definição C.1.

${ }^{2} \mathrm{O}$ conceito de classe se faz necessário toda vez que se tenta construir "conjuntos" com cardinalidade maior que o universo da teoria de conjuntos adotada (ingênua ou segundo os axiomas de ZERMELOFrAENKEL). O exemplo paradigmático, apontado pela primeira vez por B. Russell, é a classe dos subconjuntos do universo.

3 Existem certas categorias cujos objetos podem não constituir uma classe, como no caso de categorias de funtores (ver Seção C.2).
} 
1. objetos $a, b, c, \ldots$;

2. setas (ou morfismos) $f, g, h, \ldots$;

3. operações de domínio (ou fonte) $D($.$) e contradomínio (ou alvo) C D($.$) sobre as$ setas, resultando nos objetos $D(f), C D(f), D(g), C D(g), \ldots$ (é costume empregar a notação

$$
f: a \longrightarrow b \quad \text { ou } \quad a \stackrel{f}{\longrightarrow} b, \quad \text { para } \quad a=D(f), b=C D(f)) ;
$$

4. uma operação de identidade $i d$. $\equiv \mathbb{1}$. sobre os objetos, resultando nas setas $\mathbb{1}_{a}, \mathbb{1}_{b}, \ldots$ com $D\left(\mathbb{1}_{a}\right)=C D\left(\mathbb{1}_{a}\right) ;$

5. e uma operação de composição . o. sobre pares ordenados de setas $(f, g)$ com $D(f)=C D(g)$, resultando nas setas $f \circ g$ com $D(f \circ g)=D(g)$ e $C D(f \circ g)=$ $C D(f)$,

sendo as operações acima sujeitas aos seguintes axiomas:

CAt1 (Associatividade): Para quaisquer objetos e setas arranjados na forma

$$
a \stackrel{f}{\longrightarrow} b \stackrel{g}{\longrightarrow} c \stackrel{h}{\longrightarrow} d,
$$

temos que $h \circ(g \circ f)=(h \circ g) \circ f$;

Cat2 (lei unitária): $\quad$ Para qualquer seta $f$, temos que $\mathbb{1}_{C D(f)} \circ f=f \circ \mathbb{1}_{D(f)}=f .4$

Para nossos propósitos, será suficiente considerar uma categoria como sendo a seguinte realização de uma metacategoria dentro da teoria de conjuntos (ver a nota de rodapé 3$)$ :

DEFINIÇÃO C.2 Uma categoria $\mathscr{C}$ consiste de uma classe de objetos Obj $\mathscr{C}$ (por vezes identificada, por abuso de notação, com $\mathscr{C}$ ), e de uma classe de setas Arr $\mathscr{C}^{5}$, dotadas de operações satisfazendo os axiomas de uma metacategoria, tal que as subclasses $H_{\operatorname{Hom}}(a, b) \doteq\{f: D(f)=a, C D(f)=b\}$ são conjuntos, para todo par $a, b \in O b j \mathscr{C}$.

\footnotetext{
${ }^{4}$ Como este axioma determina $\mathbb{1}_{a}$ completamente para qualquer objeto $a$, é por vezes conveniente identificar $a$ com a seta $\mathbb{1}_{a}$. Em particular, isso torna possível definir uma metacategoria apenas por meio de setas (ver [Lan98] para uma discussão detalhada).

${ }^{5}$ Esta pode, em virtude da discussão na nota de rodapé 4 , ser considerada como uma superclasse de $O b j \mathscr{C}$. Sob este ponto de vista, seria mais adequado identificar $\mathscr{C}$ com $\operatorname{Arr} \mathscr{C}$, mas aqui nos rendemos à prática corrente.
} 
Uma subcategoria $\mathscr{D}$ de $\mathscr{C}$ consiste de uma categoria cujos objetos formam uma subclasse $\operatorname{Obj} \mathscr{D}$ de $\operatorname{Obj} \mathscr{C}$ e as setas formam uma subclasse $\operatorname{Arr} \mathscr{D}$ de $\operatorname{Arr} \mathscr{C}$, tal que as operações de $\mathscr{D}$ são herdadas de $\mathscr{C}$. $\mathscr{D}$ é uma subcategoria plena se $\operatorname{Hom}_{\mathscr{D}}(a, b)=$ $\operatorname{Hom}_{\mathscr{C}}(a, b), \forall a, b \in \mathscr{D}$. Dizemos também que uma categoria $\mathscr{C}$ é pequena se $\operatorname{Obj} \mathscr{C}_{\text {(e, }}$ portanto, $\operatorname{Arr} \mathscr{C}$ ) for um conjunto. Podemos associar a $\mathscr{C}$ a categoria oposta $\mathscr{C}^{o p}$ tal que $\operatorname{Obj} \mathscr{C}^{o p}=\operatorname{Obj} \mathscr{C}_{\text {e } H_{o m} o p}(a, b)=\operatorname{Hom}_{\mathscr{C}}(b, a)$, com a lei de composição o invertida em relação a $\mathscr{C}$.

Uma vez que as setas abstraem da categoria $\mathscr{S}$ et de conjuntos a noção de funções entre conjuntos, é natural buscar a abstração categórica das funções injetoras, sobrejetoras e bijetoras. Dizemos que uma seta $f$ é sobrejetora, épica ou simplesmente uma epi (resp. injetora, mônica ou uma mono) se dadas duas setas $g_{1}, g_{2} \in \operatorname{Hom}_{\mathscr{C}}(C D(f), b)$ (resp. $h_{1}, h_{2} \in \operatorname{Hom}_{\mathscr{C}}(a, D(f))$ ) tais que $g_{1} \circ f=g_{2} \circ f$ (resp. $f \circ h_{1}=f \circ h_{2}$ ), temos que $g_{1}=g_{2}$ (resp. $\left.h_{1}=h_{2}\right)^{6}$. Uma seta e é inversível à direita (resp. esquerda) se existe uma seta $e^{\prime}$ tal que $D\left(e^{\prime}\right)=C D(e), C D\left(e^{\prime}\right)=D(e)$ e satisfazendo $e \circ e^{\prime}=\mathbb{1}_{C D(e)}$ (resp. $e^{\prime} \circ e=\mathbb{1}_{D(e)}$ ), e simplesmente inversível (ou um isomorfismo) se ambas as propriedades são satisfeitas e toda a inversa à direita o for à esquerda e vice-versa. Neste caso, a inversa é única, o que não ocorre nos casos anteriores. Também dizemos neste caso que $D(e)$ e $C D(e)$ são isomorfos. Um grupóide é uma categoria pequena tal que toda seta é um isomorfismo.

Sejam $f$ epi e $g$ mono. Se $f \circ g=\mathbb{1}_{D(g)}$, é praxe dizer que $g$ é uma seção de $f$ (em analogia com seções de fibrados), e $f$ uma retração de $g$. Também aqui dizemos que $f$ é uma epi cindida e $g$, uma mono cindida. Neste caso, $h=g \circ f: D(f) \longrightarrow D(f)$ é uma seta idempotente (ou uma projeção), i.e., $h^{2} \doteq h \circ h=h$. Conversamente, uma seta idempotente $h \in \operatorname{Hom}_{\mathscr{C}}(a, a)$ é dita cindida por um objeto $b$ se existem uma epi $f: a \longrightarrow b$ e uma mono $g: b \longrightarrow a$ tais que $f \circ g=\mathbb{1}_{b}$ e $g \circ f=h$.

Por fim, passemos a alguns exemplos, que também nos servirão para fixar a notação para algumas categorias padrão que serão utilizadas com freqüência:

1: $\quad$ Categoria dotada de um único objeto a e uma única seta $\mathbb{1}_{a}$;

2: $\quad$ Categoria dotada de apenas dois objetos $a, b$ e três setas $\mathbb{1}_{a}, \mathbb{1}_{b}, a \stackrel{f}{\longrightarrow} b$;

\footnotetext{
${ }^{6}$ Não se buscou definir setas épicas (resp. mônicas) por meio de inversas à direita (resp. esquerda) pois, embora tal definição se aplique a $\mathscr{S}$ et, o mesmo não ocorre, por exemplo, com a categoria $\mathscr{G} r p$ de grupos - epimorfismos possuem uma inversa conjuntística à direita, mas pode não ser possível escolher um homomorfismo com essa propriedade. A definição que demos, por outro lado, comporta todos os casos de interesse.
} 
$U: \quad$ Categoria (pequena) discreta, onde $\operatorname{Obj} U=U$ é um conjunto, $\operatorname{Hom}_{U}(a, b)=\varnothing$ se $a \neq b$, e $\operatorname{Hom}_{U}(a, a)\left\{\mathbb{1}_{a}\right\}$ (em suma, podemos também escrever $U=O b j U=$ $\operatorname{Arr} U)$;

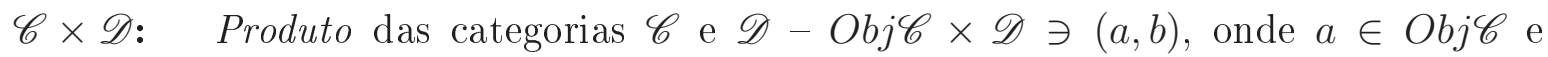

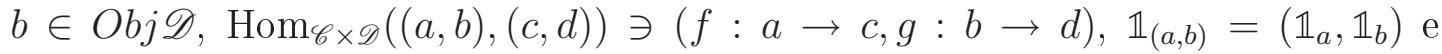
$(f, g) \circ\left(f^{\prime}, g^{\prime}\right)=\left(f \circ f^{\prime}, g \circ g^{\prime}\right)$;

$\mathscr{C}^{I}$ : Potência Cartesiana de uma categoria $\mathscr{C}$ pelo conjunto $I$ - formalmente, $\operatorname{Obj} \mathscr{C} \ni_{\ni}$

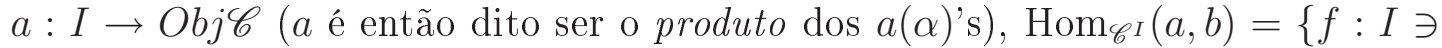
$\left.\alpha \mapsto f(\alpha) \in \operatorname{Hom}_{\mathscr{C}}(a(\alpha), b(\alpha))\right\}, \mathbb{1}_{a}: I \ni \alpha \mapsto \mathbb{1}_{a(\alpha)}$ e $(f \circ g): I \ni \alpha \mapsto f(\alpha) \circ g(\alpha) ;$

$(U, \leq): \quad$ Conjunto parcialmente ordenado $-\operatorname{Obj}(U, \leq)=U$ é o conjunto subjacente, e $\mathscr{H}_{(U, \leq)}(a, b)$, se não vazio, possui uma única seta $\leq$, denotada também por $a \leq b$, satisfazendo as propriedades usuais de uma relação de ordem, aqui devidamente refraseadas em linguagem categórica: $\mathbb{1}_{a}=a \leq a$ e $(b \leq c) \circ(a \leq b)=a \leq c$. Neste caso, $\operatorname{Arr}(U, \leq) \doteq \leq$ é dita ser uma ordem parcial em $U$. Dizemos destarte que $(U, \leq)$ é: direcionado se, dados $a, b \in U$, existe $c \in U$ tal que $a \leq c$ e $b \leq c$; e totalmente ordenado se para quaisquer $a, b \in U$ necessariamente $a \leq b$ ou $b \leq a$. O Lema de ZORN aqui fica enunciado da seguinte forma: qualquer subcategoria $\mathscr{C}$ plena e totalmente ordenada de $(U, \leq)$ possui um objeto terminal $u$, i.e., para todo $a \in \mathscr{C}$ existe uma única seta $a \leq u$ (dualmente, $a \in$ Obj $\mathscr{C}$ é um objeto inicial se para todo $b \in O b j \mathscr{C}$ existe uma única seta $a \rightarrow b)$. Um exemplo de conjunto direcionado é o conjunto das partes ordenado por inclusões $(U, \leq)=(P(X), \subseteq)$ do conjunto $X-$ em particular, esse conjunto direcionado possui um objeto terminal $X$ e objetos iniciais $\{x\}, x \in X$;

$\mathscr{S}$ et: $\quad$ Conjuntos, cujas setas são funções;

$\mathscr{G}_{r p:}$ Grupos, cujas setas são homomorfismos;

Top: $\quad$ Espaços topológicos, cujas setas são funções contínuas;

Tvs: Espaços vetoriais topológicos (no texto assumidos HAUsDorfF e localmente convexos - ver Seção B.3), cujas setas são aplicações lineares contínuas.

Outras categorias serão introduzidas no texto à medida da necessidade.

\section{C.2 Funtores e transformações naturais}

Definição C.3 Sejam as categorias $\mathscr{A}$ e $\mathscr{B}$. Um funtor covariante (resp. contravariante) de $\mathscr{A}$ em $\mathscr{B}$ é uma regra $\mathfrak{F}$ que associa a cada a $\in$ Obj $\mathscr{A}$ um objeto $\mathfrak{F}$ a $\in$ Obj $\mathscr{B}$ e a cada $f \in \operatorname{Arr} \mathscr{A}$ uma seta $\mathfrak{F} f \in \operatorname{Arr} \mathscr{B}$ satisfazendo $D(\mathfrak{F} f)=\mathfrak{F} D(f)$ (resp. 
$D(\mathfrak{F} f)=\mathfrak{F} C D(f)), C D(\mathfrak{F} f)=\mathfrak{F} C D(f)$ (resp. $C D(\mathfrak{F} f)=\mathfrak{F} D(f)$ ) e tal que, para $a, b, c \in \operatorname{Obj} \mathscr{A}$ e $f, g \in \operatorname{Arr} \mathscr{A}$ satisfazendo $a \stackrel{f}{\longrightarrow} b \stackrel{g}{\longrightarrow}$, temos que

$$
\mathfrak{F}(g \circ f)=\mathfrak{F} g \circ \mathfrak{F} f(r e s p . ~ \mathfrak{F}(g \circ f)=\mathfrak{F} f \circ \mathfrak{F} g) .
$$

É praxe escrever o funtor como uma "seta entre categorias", i.e., $\mathfrak{F}: \mathscr{A} \rightarrow \mathscr{B}$ ou mesmo $\mathscr{A} \stackrel{\mathfrak{F}}{\longrightarrow} \mathscr{B}$.

Podemos, equivalentemente, dizer que um funtor contravariante $\mathfrak{F}: \mathscr{A} \rightarrow \mathscr{B}$ é um funtor covariante $\mathfrak{F}: \mathscr{A} \rightarrow \mathscr{B}^{o p}$. Assim, quando dizemos funtor, queremos dizer apenas "funtor covariante".

$\mathfrak{F}$ é dito ser uma equivalência de categorias se for inversível, i.e., existe um funtor $\mathfrak{F}^{-1}$ tal que $\mathfrak{F}^{-1} \circ \mathfrak{F}=\mathfrak{i d} \mathfrak{A}_{\mathscr{A}}$ e $\mathfrak{F} \circ \mathfrak{F}^{-1}=\mathfrak{i d} \mathfrak{d}_{\mathscr{B}}$, onde $\mathscr{C} \stackrel{\mathfrak{i} \mathfrak{C} \mathscr{C}}{\longrightarrow} \mathscr{C}$ é o funtor identidade associado

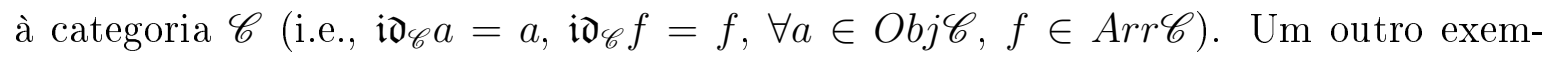
plo é o funtor $\mathfrak{i}_{\mathscr{C}, \mathscr{D}}$ dado pela inclusão de uma categoria $\mathscr{C}$ numa supercategoria $\mathscr{D}$ de $\mathscr{C}$.

Daremos agora alguns exemplos de funtores que nos serão de grande utilidade:

- Sejam $\mathscr{C}$ uma categoria e $(U, \leq)$ um conjunto parcialmente ordenado. Um funtor $\mathfrak{F}:(U, \leq) \rightarrow \mathscr{C}^{o p}$ (resp. $\left.\mathfrak{G}:(U, \leq) \rightarrow \mathscr{C}\right)$ é dito ser um pré-(co)feixe (de objetos de $\mathscr{C})$ em $U \cdot^{7}$ Se $(U, \leq)$ for direcionado, dizemos que $\mathfrak{F}$ é um sistema inverso em $\mathscr{C}$ indexado por $U$, e $\mathfrak{G}$, uma rede (de objetos de $\mathscr{C}$ ) em $U$, ou um sistema direto em $\mathscr{C}$ indexado por $U$;

- A potência Cartesiana de $\mathscr{C}$ por $I$ pode ser entendida como um funtor $\mathfrak{F}: I \rightarrow \mathscr{C}$, onde $I$ aqui é entendido como uma categoria (discreta);

- Sejam $I$ um conjunto e $\mathscr{C}$ uma categoria. O funtor diagonal $\Delta: \mathscr{C} \rightarrow \mathscr{C}^{I}$ é dado por $\operatorname{Obj}_{\mathscr{C}} \ni a \mapsto(\Delta a: I \ni \alpha \mapsto a)$ e $\operatorname{Arr} \mathscr{C} \ni f \mapsto(\Delta f: I \ni \alpha \mapsto f)$;

- Dado um funtor $\mathfrak{F}: \mathscr{C} \rightarrow \mathscr{D}$ e $d \in$ Obj $\mathscr{D}$, uma seta universal de $d$ em $\mathfrak{F}$ (resp. de $\mathfrak{F}$ em $d$ ) consiste num par $(u, f)$, onde $u \in O b j \mathscr{C}$ e $f: d \rightarrow \mathfrak{F} u($ resp. $f: \mathfrak{F} u \rightarrow d$ )

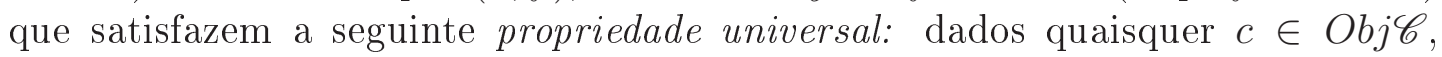
$g: d \rightarrow \mathfrak{F} c$ (resp. $g: \mathfrak{F} c \rightarrow d)$, existe uma única seta $g^{\prime}: u \rightarrow c\left(\right.$ resp. $g^{\prime}: c \rightarrow u$ ) tal que $\mathfrak{F} g^{\prime} \circ f=g$ (resp. $\left.f \circ \mathfrak{F} g^{\prime}=g\right)$. Neste caso, dizemos que $(u, f)$ é um elemento universal do funtor $\operatorname{Hom}_{\mathscr{D}}(d, \mathfrak{F}$.) : $\mathscr{C} \rightarrow \mathscr{S}$ et (a ação deste funtor sobre setas é dada por $\left.\operatorname{Hom}_{\mathscr{D}}(d, \mathfrak{F}):.(g: a \rightarrow b) \mapsto\left(\operatorname{Hom}_{\mathscr{D}}(d, \mathfrak{F} a) \ni h \mapsto \mathfrak{F} g \circ h \in \operatorname{Hom}(d, \mathfrak{F} b)\right)\right)$ - mais em geral, se $\mathfrak{H}: \mathscr{C} \rightarrow \mathscr{S}$ et, um objeto universal de $\mathfrak{H}$ consiste do par $(u, e)$,

\footnotetext{
${ }^{7} \mathrm{Na}$ verdade, a definição usual de pré-(co)feixe em $U$ consiste num funtor $\mathscr{F}:(P(U), \subseteq) \rightarrow \mathscr{C}$ ou, mais em geral, $\mathfrak{F}:(\tau(U), \subseteq) \rightarrow \mathscr{C}$, onde $\tau$ é uma topologia (possivelmente discreta, como no caso anterior) em $U$. Não é difícil ver que tal definição é englobada pela que demos acima.
} 
onde $u \in O b j \mathscr{C}$ e $e \in \mathfrak{H} u$ são tais que para todo $a \in O b j \mathscr{C}, x \in \mathfrak{H} a$ existe uma única seta $f: u \rightarrow a$ tal que $(\mathfrak{H} f)(e)=x$. Conversamente, interpretando $e$ como $e:\{*\} \ni * \mapsto(\mathfrak{H} u)(*) \doteq e$, vemos que $(u, e)$ é uma seta universal de $*$ em $\mathfrak{H}$. O caso dual citado paralelamente acima consiste apenas em trocar $\mathscr{D}$ por $\mathscr{D}^{o p}$. Exemplos de setas universais compreendem:

- Geradores de grupos livres $\left(\mathscr{C}=\mathscr{G}_{r p}, \mathscr{D}=\mathscr{S}\right.$ et e $\mathfrak{F}$ identifica um grupo com o conjunto subjacente): dado $X \in O b j \mathscr{S}$ et, seja $u$ o grupo livre gerado por $X$ e $f$ identificando $x \in X$ com o elemento correspondente de $u$;

- Coprodutos $\left(\mathscr{D}=\mathscr{C}^{I}\right.$, onde $I$ é um conjunto, e $\left.\mathfrak{F}=\Delta\right)$ : dado $a: I \ni$ $\alpha \mapsto a(\alpha)$ e $(u, f)$ uma seta universal de $a$ em $\Delta$, dizemos então que $u$ é o coproduto ou soma direta dos $a(\alpha)$ 's, por vezes denotado $u \doteq \coprod_{\alpha \in I} a(\alpha)$ ou $u \doteq \bigoplus_{\alpha \in I} a(\alpha)$, e $f(\alpha)$, a injeção de $a(\alpha)$ em $u$;

- Produtos $\left(\mathscr{D}=\mathscr{C}^{I}\right.$, onde $I$ é um conjunto, e $\left.\mathfrak{F}=\Delta\right)$ : dado $a: I \ni \alpha \mapsto a(\alpha)$ e $(u, f)$ uma seta universal de $\Delta$ em $a$, dizemos então que $u$ é o produto dos $a(\alpha)$ 's, por vezes denotado $u \doteq \prod_{\alpha \in I} a(\alpha)$, e $f(\alpha)$, a projeção de $u$ em $a(\alpha)$;

- Completamento de espaços topológicos, álgebras tensoriais livres, etc.,

e de objetos universais:

- Quociente $S / \sim$ de um conjunto $S$ por uma relação de equivalência $\sim(\mathscr{C}=$ Set, $\left.\mathfrak{H}: X \mapsto \mathfrak{H} X=\left\{f: S \rightarrow X: s, s^{\prime} \in S, s \sim s \Rightarrow f(s)=f\left(s^{\prime}\right)\right\}\right) ;$

- Produto tensorial de dois espaços vetoriais (caso particular do exemplo anterior), etc..

- Dada uma categoria $\mathscr{C}$, um conjunto direcionado $(U, \leq)$ e um sistema direto (resp. inverso) $\mathfrak{F}$ em $\mathscr{C}$ indexado por $U$, o limite indutivo (resp. projetivo),

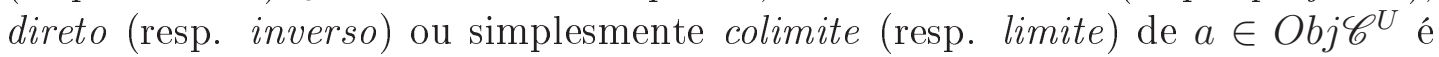
simplesmente uma seta universal $(u, f)$ de $a$ em $\Delta$ (resp. de $\Delta$ em $a$ ), tal que $f\left(\alpha^{\prime}\right)=f(\alpha) \circ \mathfrak{F}\left(\alpha^{\prime} \leq \alpha\right)$ (resp. $\left.f\left(\alpha^{\prime}\right)=\mathfrak{F}\left(\alpha^{\prime} \leq \alpha\right) \circ f(\alpha)\right)-$ denotamos

$$
u \doteq \lim _{\alpha, \vec{F}} a(\alpha)(\operatorname{resp} . u \doteq{\underset{\lim }{\alpha, \mathfrak{F}}} a(\alpha))
$$

DefiniçÃo C.4 Sejam as categorias $\mathscr{A}$ e $\mathscr{B}$, e os funtores ${ }^{8} \mathfrak{F}, \mathfrak{G}: \mathscr{A} \rightarrow \mathscr{B}$. Uma transformação natural $\Phi$ de $\mathfrak{F}$ em $\mathfrak{G}$ é uma regra que associa a cada $a \in$ Obj $\mathscr{A}$ uma seta $\Phi_{a} \in \operatorname{Arr} \mathscr{B}$ satisfazendo $D\left(\Phi_{a}\right)=\mathfrak{F} a, C D\left(\Phi_{a}\right)=\mathfrak{G} a$ e tal que, para toda $f: a \rightarrow b$, $a, b \in \operatorname{Obj} \mathscr{A}$, temos que

$$
\Phi_{a} \circ \mathfrak{F} f=\mathfrak{G} f \circ \Phi_{b}
$$


Uma transformação natural $\phi$ entre funtores $\mathfrak{F}, \mathfrak{G}$ é dita ser uma equivalência natural entre $\mathfrak{F}$ e $\mathfrak{G}$ se existe uma transformação natural $\phi^{-1}$ de $\mathfrak{G}$ em $\mathfrak{F}$ tal que $\phi_{a}^{-1} \circ \phi_{a}=\phi_{a} \circ \phi_{a}^{-1}=\mathbb{1}_{a}$. Por exemplo, um funtor $\mathfrak{F}$ de uma categoria $\mathscr{C}$ numa supercategoria $\mathscr{D}$ de $\mathscr{C}$ é dito ser esquecido se for naturalmente equivalente ao funtor de inclusão de $\mathscr{C}$ em $\mathscr{D}$, i.e., $\mathfrak{F}$ "esquece" parte da estrutura de $\mathscr{C}$.

Uma vez que a composição de transformações naturais é claramente associativa, e sempre existe, para cada funtor $\mathfrak{F}$, uma identidade $\mathbb{1}_{\mathfrak{F}}$ dada por $\left(\mathbb{1}_{\mathfrak{F}}\right)_{a} \doteq \mathbb{1}_{\mathfrak{F} a}$, podemos considerar formalmente a categoria de funtores $\mathscr{C}^{\mathscr{D}}$, com $\operatorname{Obj}_{\mathscr{C}} \mathscr{D}=\{\mathfrak{F}: \mathscr{D} \rightarrow \mathscr{C}\}$ e $\operatorname{Arr} \mathscr{C}^{\mathscr{D}}$ consistindo de todas as transformações naturais entre funtores de $\mathscr{D}$ em $\mathscr{C}$, modulo as considerações conjuntísticas no início deste Apêndice, em particular a nota de rodapé 3 . Um exemplo de tal categoria é, dada uma categoria $\mathscr{A}$, a categoria de setas $\mathscr{A}^{2}$.

\footnotetext{
${ }^{8}$ Usando a noção de categoria oposta, podemos estender a definição para funtores contravariantes.
} 



\section{APÊNDICE D}

\section{Rudimentos de homotopia}

Introduzimos aqui algumas definições e resultados básicos que são empregados em vários pontos do Capítulo 1. Nossa referência para o assunto é a Parte I (Seções 1-7) de [GH81]. Para as aplicações relacionadas a variedades diferenciáveis (isotopias, etc.), seguimos [Hir76].

\section{D.1 Aplicações homotópicas}

DEFINIÇÃo D.1 Dados dois espaços topológicos $X, Y$ e duas aplicações contínuas $f, g$ : $X \rightarrow Y$, dizemos que $f$ é homotópica a $g$ se existe uma aplicação (juntamente) contínua $F: X \times[0,1] \rightarrow Y$ tal que $F(x, 0)=f(x)$ e $F(x, 1)=g(x)$, para todo $x \in X$ (tal $F$ é dita uma homotopia entre $f$ e g). Em particular, se $A \subset X$ e $f \uparrow_{A}=g \uparrow_{A}$, dizemos que $f$ é homotópica a $g$ em relação a $A$ se podemos escolher a homotopia $F$ de maneira tal que $F(x, t)=f(x)=g(x)$ para todo $t \in[0,1]$ - neste caso, dizemos que $F$ é uma homotopia entre $f$ e $g$ em relação a $A$.

Dizemos que $X$ é contrátil se a aplicação identidade $\operatorname{id}_{X}: X \ni x \mapsto x$ é homotópica à aplicação constante $f_{p}(x): X \ni x \mapsto p$ para algum $p \in X$. X é contrátil se e somente se, dado qualquer espaço topológico $Y$, quaisquer duas aplicações contínuas $f, g: Y \rightarrow X$ são homotópicas - a implicação direta é imediata, e a implicação inversa é obtida através da homotopia $G: Y \times[0,1] \rightarrow X$ dada por $G(y, t)=F(f(y), 2 t)$ $\left(t \in\left[0, \frac{1}{2}\right]\right)$ e $G(y, t)=F(g(y), 1-2 t)\left(t \in\left[\frac{1}{2}, 1\right]\right)$, onde $F$ é uma homotopia entre id $X$ e $f_{p}$. Em conseqüência disto, um espaço topológico contrátil $X$ é sempre conexo por caminhos, i.e., dados quaisquer pontos $x, y \in X$, existe uma curva contínua ligando $x$ a $y$ - a saber, $\gamma:[0,1] \rightarrow X$ dada por $\gamma(t)=F(x, 2 t)\left(t \in\left[0, \frac{1}{2}\right]\right)$ e $\gamma(t)=F(y, 1-2 t)$ $\left(t \in\left[\frac{1}{2}, 1\right]\right)$.

Um espaço topológico contrátil $X$ é também simplesmente conexo, i.e., quaisquer duas curvas $\gamma, \gamma^{\prime}:[0,1] \ni s \rightarrow X$ tais que $\gamma(0)=\gamma^{\prime}(0)$ e $\gamma(1)=\gamma^{\prime}(1)$ são homotópicas 
com extremos fixos, i.e., homotópicas em relação a $\{0,1\}$.

Consideremos agora duas variedades diferenciáveis $\mathscr{M}, \mathscr{N}$. Dadas duas aplicações $\mathscr{C}^{\infty} f, g: \mathscr{M} \rightarrow \mathscr{N}$ e uma homotopia $F$ entre $f$ e $g$, sempre é possível regularizar $F$ de modo tal que $F$ é uma aplicação $\mathscr{C}^{\infty}$ de $\mathscr{M} \times[0,1]$ em $\mathscr{N}$. O mesmo vale se $f=g$ num subconjunto $U \subset \mathscr{M}$ e $F$ é uma homotopia entre $f$ e $g$ em relação a $U$. Assim, uma vez transportado o conceito de homotopia da categoria $\mathscr{C}^{0}$ para a categoria $\mathscr{C}^{\infty}$, podemos refiná-lo da seguinte forma:

- Se $F(., t)$ é um mergulho de $\mathscr{M}$ em $\mathscr{N}$ para todo $t \in[0,1]$ (e, em particular, $f$ e $g$ também o são), dizemos que $F$ é uma isotopia entre $f$ e $g$. Um exemplo típico de isotopia é dado por uma folheação de $K \subset \mathscr{N}$ por cópias difeomorfas de $\mathscr{M}$ neste caso, $K=F(\mathscr{M},[0,1])$ e $F\left(\mathscr{M}, t_{1}\right) \cap F\left(\mathscr{M}, t_{2}\right)=\varnothing$ se $t_{1} \neq t_{2}$.

- Se $\mathscr{M}=\mathscr{N}, F(., t)$ é um difeomorfismo para todo $t \in[0,1]$ e $f=\mathrm{id}_{\mathscr{M}}$, $\operatorname{dizemos}$ que $F$ é uma difeotopia ou isotopia ambiente. Um exemplo de difeotopia é a restrição de um grupo uniparamétrico de difeomorfismos $\mathscr{M} \times \mathbb{R} \ni(x, t) \mapsto \phi_{t}(x)$ (i.e., $\phi_{t_{1}+t_{2}}=\phi_{t_{1}} \circ \phi_{t_{2}}$ e $\phi_{0}=\mathrm{id}_{\mathscr{M}}$ ) a $\mathscr{M} \times[0,1]-$ neste caso, $g=\phi_{1}$.

\section{D.2 O grupo fundamental e espaços de recobrimento}

Definição D.2 Seja $X$ um espaço topológico e $x_{0} \in X$, denominado base. $O$ espaço de laços $\Omega\left(X, x_{0}\right)$ de $X$ baseado em $x_{0}$ consiste das curvas continuas $\gamma:[0,1] \rightarrow X$ tais que $\gamma(0)=\gamma(1)=x_{0}$. Considere a seguinte relação de equivalência $\sim$ em $\Omega\left(X, x_{0}\right)$ :

$$
\gamma_{1} \sim \gamma_{2} \Leftrightarrow \gamma_{1} \text { é homotópica a } \gamma_{2} \text { com extremos fixos, }
$$

as operações de

- Produto: $\gamma_{1}, \gamma_{2} \in \Omega\left(X, x_{0}\right) \rightarrow \gamma_{1} \gamma_{2}$ dada por

$$
\gamma_{1} \gamma_{2}(t)=\left\{\begin{array}{ll}
\gamma_{1}(2 t) & \left(t \in\left[0, \frac{1}{2}\right]\right) \\
\gamma_{2}(2 t-1) & \left(t \in\left[\frac{1}{2}, 1\right]\right)
\end{array}, e\right.
$$

- Inversão: $\gamma^{-1}(t)=\gamma(1-t)$

e o laço identidade $\gamma_{0}(t) \equiv x_{0}$ em $\Omega\left(X, x_{0}\right)$. $O$ grupo fundamental (ou primeiro grupo de homotopia) de $X$ baseado em $x_{0}$ é dado conjuntisticamente por $\pi_{1}\left(X, x_{0}\right)=$ $\Omega\left(X, x_{0}\right) / \sim \ni[\gamma]$, e com operações de grupo $\left[\gamma_{1}\right]\left[\gamma_{2}\right] \doteq\left[\gamma_{1} \gamma_{2}\right],[\gamma]^{-1} \doteq\left[\gamma^{-1}\right]$ e identidade $\mathbb{1} \doteq\left[\gamma_{0}\right]$ 
Claramente, a componente conexa por caminhos de $X$ à qual $x_{0}$ pertence é simplesmente conexa se e somente se $\pi_{1}\left(X, x_{0}\right)=\{\mathbb{1}\}$. Se $X$ é conexo por caminhos, então $\pi_{1}\left(X, x_{1}\right)$ é isomorfo a $\pi_{1}\left(X, x_{2}\right)$ para todo $x_{1}, x_{2} \in X$, e podemos então definir o grupo fundamental $\pi_{1}(X) \doteq \pi_{1}\left(X, x_{0}\right)$ de $X$.

DEFINIÇÃo D.3 Dados dois espaços topológicos $X, Y$, uma aplicação $\phi: Y \rightarrow X$ é dita de recobrimento se cada $x \in X$ possui uma vizinhança aberta $U \ni x$ tal que $\phi^{-1}(U)=\dot{U}_{\alpha} V_{\alpha}$, onde $\left\{V_{\alpha}\right\}$ é uma coleção de abertos disjuntos (Uं denota união disjunta) de $Y$ tais que $\phi \uparrow_{V_{\alpha}}$ é um homeomorfismo tal que $F\left(V_{\alpha}\right)=U$, para todo $\alpha$. Neste caso dizemos que $Y$ é um (espaço de) recobrimento de $X$ - empregamos também a notação condensada $Y \stackrel{\phi}{\longrightarrow} X . Y$ é dito universal se for conexo por caminhos $e$ simplesmente conexo.

Se $Y \stackrel{\phi}{\longrightarrow} X$ é um recobrimento do espaço topológico $X$, segue imediatamente da Definição D.3 que:

(i) $\phi^{-1}(x)$ é discreto para todo $x \in X$,

(ii) $\phi$ é um homeomorfismo local, e

(iii) $\phi$ é sobrejetora, e a topologia de $X$ é precisamente a topologia quociente de $E$ pela relação de equivalência $y_{1} \sim y_{2} \Leftrightarrow \phi\left(y_{1}\right)=\phi\left(y_{2}\right)$.

A importância do recobrimento universal decorre do fato de que, apesar de ter uma estrutura global bastante simples, ele codifica de maneira bastante conveniente o grupo fundamental do espaço topológico que recobre. Definindo o grupo das transformações de recobrimento $G(Y, X)$ de um recobrimento universal $Y$ de $X$ como o grupo dos homeomorfismos $f$ de $Y$ tais que $\phi \circ f=\phi$, pode-se provar [GH81] que $G(Y, X) \cong \pi_{1}(X)$.

Mais ainda, o recobrimento universal $Y$ de $X$ é essencialmente único: dado qualquer outro recobrimento universal $Y^{\prime} \stackrel{\phi^{\prime}}{\longrightarrow} X$, existe um homeomorfismo $f: Y^{\prime} \rightarrow Y$ tal que $\phi \circ f=\phi^{\prime}$. Podemos então falar do recobrimento universal de $X$. Conversamente, dado um grupo $G$ de homeomorfismos de um espaço topológico conexo por caminhos e simplesmente conexo $Y$ que age propriamente descontinuamente, i.e., para todo $y \in Y$ existe uma vizinhança aberta $V \ni y$ tal que $f(V) \cap V=\varnothing$ para todo $f \in G$, segue que $\pi_{1}(Y / G) \cong G$ - note que, acima, $G(Y, X)$ age propriamente descontinuamente em $Y$. Dizemos então que $X=Y / G$ é o dominio fundamental de $Y$ com respeito a $G$. 



\section{Referências Bibliográficas}

[Ae73] H. Araki, G. Elliott. On the Definition of $C^{*}$-Algebras. Publ. RimS 9 (1973) 93-112.

[AGH98a] L. Andersson, G. J. Galloway, R. Howard. A Strong Maximum Principle for Weak Solutions of Quasi-Linear Elliptic Equations with Applications to Lorentzian and Riemannian Geometry. Commun. Pure Appl. Math. L 1 (1998) 581-624. arXiv:dg-ga/9707015.

[AGH98b] - The Cosmological Time Function. Clas. Quantum Grav. 15 (1998) 309-322. arXiv: gr-qc/9709084.

[And05] M. T. Anderson, Unique Continuation Results for Ricci Curvature and Applications (2005). Preprint, arXiv:math .DG/0501067.

[And06a] -, Boundary Value Problems for Einstein Metrics, I (2006). Preprint, arXiv:math.DG/0612647.

[And06b] - On the Uniqueness and Global Dynamics of AdS Spacetimes. Clas. Quantum Grav. 23 (2006) 6935-6954. arXiv: hep-th/0605293.

[And06c] - Topics in Conformally Compact Einstein metrics. In: Perspectives in Riemannian Geometry, CRM Proc. Lecture Notes, vol. 40, 2006, 1-26. arXiv: math. DG . 0503243.

[Ara76] H. Araki. Relative Entropy of States of von Neumann Algebras. Publ. RIMS 11 (1976) 809-833.

[Ara99] - Mathematical Theory of Quantum Fields. Oxford University Press, 1999.

[Arn89] V. I. ARnold, Mathematical Methods of Classical Mechanics. 2nd. ed., Springer-Verlag, 1989.

[AS80] A. Ashtekar, A. Sen. On the Role of Space-Time Topology in Quantum Phenomena: Superselection of Charge and Emergence of Nontrivial Vacua. J. Math. Phys. 21 (1980) 526-533.

[AS02] M. Arnsdorf, L. Smolin, The Maldacena Conjecture and Rehren Duality (2002). Preprint, arXiv: hep-th/0106073. 
[BBMS00] M. Bertola, J. Bros, U. Moschella, R. Schaeffer. AdS/CFT Correspondence for n-point Functions. Nucl. Phys. B 587 (2000) 619-644. arXiv: hep-th/9908140.

[BCH73] J. M. Bardeen, B. Carter, S. W. Hawking. The Four Laws of Black Hole Mechanics. Commun. Math. Phys. 31 (1973) 161-170.

[BEE96] J. K. Beem, P. E. Ehrlich, K. L. Easley, Global Lorentzian Geometry. 2nd. ed., Marcel Dekker, 1996.

[Bek74] J. D. Bekenstein. Generalized Second Law of Thermodynamics in BlackHole Physics. Phys. Rev. D 9 (1974) 3292-3300.

[BF07] R. Brunetti, K. Fredenhagen, Towards a Background Independent Formulation of Perturbative Quantum Gravity. In: Proceedings of the "Workshop on Mathematical and Physical Aspects of Quantum Gravity", Birkhäuser, Blaubeuren, 2007, 151-159. arXiv:gr-qc/0603079.

[BFK96] R. Brunetti, K. Fredenhagen, M. Köhler. The Microlocal Spectrum Condition and Wick Polynomials of Free Fields on Curved Spacetimes. Commun. Math. Phys. 180 (1996) 633-652. arXiv:gr-qc/9510056.

[BFS00] D. Buchholz, M. Florig, S. J. Summers. The Second Law of Thermodynamics, TCP, and Einstein Causality in Anti-de Sitter Space-Time. Clas. Quantum Grav. 17 (2000) L31-L37. arXiv:hep-th/9905178.

[BFv03] R. Brunetti, K. Fredenhagen, R. Verch. The Generally Covariant Locality Principle - A New Paradigm for Local Quantum Physics. Commun. Math. Phys. 237 (2003) 31-68. arXiv:math-ph/0112041.

[BGL93] R. Brunetti, D. Guido, R. Longo. Modular Structure and Duality in Conformal Quantum Field Theory. Commun. Math. Phys. 156 (1993) 201-219. arXiv: funct-an/9302008.

[BGM71] M. Berger, P. Gauduchon, E. Mazet, Le Spectre d'une Varieté Riemannienne, Lecture Notes in Mathematics, vol. 194. Springer-Verlag, 1971.

[Bla85] B. Blackadar. Shape Theory for $C^{*}$-Algebras. Math. Scand. 56 (1985) 249-275.

[Bla06] - Operator Algebras - Theory of $C^{*}$-Algebras and von Neumann Algebras, Encyclopaedia of Mathematical Sciences, vol. 122. SpringerVerlag, 2006.

[BMT88] D. Buchholz, G. Mack, I. Todorov. The Current Algebra on the Circle as a Germ of Local Field Theories. Nucl. Phys. B (Proc. Suppl.) 5B (1988) 20-56. 
[Bor62] H.-J. Borchers. On Structure of the Algebra of Field-Operators. Nuovo Cimento 24 (1962) 214-236.

[Bor65] - On the Structure of the Algebra of Field-Operators II. J. Math. Phys. 1 (1965) 49-56.

[BOR02] D. Buchnolz, I. Ojima, H. Roos. Thermodynamic Properties of Nonequilibrium States in Quantum Field Theory. Ann. Phys. (N.Y.) 297 (2002) 219-242. arXiv: hep-ph/0105051.

[Bos05] H. Bostelmann. Operator Product Expansions as a Consequence of Phase Space Properties. J. Math. Phys. 46 (2005) 082.304.

[BR87] O. Bratteli, D. W. Robinson, Operator Algebras and Quantum Statistical Mechanics I-C*- and $W^{*}$-Algebras, Symmetry Groups, Decomposition of States. 2nd. ed., Springer-Verlag, 1987.

[BR97] -, Operator Algebras and Quantum Statistical Mechanics II - Equilibrium States, Models in Quantum Statistical Mechanics. 2nd. ed., SpringerVerlag, 1997.

[BR02] R. Bousso, L. Randall. Holographic Domains of Anti-de Sitter Space. JHEP 0204 (2002) 057. arXiv:hep-th/0112080.

[BR07] R. Brunetti, G. Ruzzi. Superselection Sectors and General Covariance. I. Commun. Math. Phys. 270 (2007) 69-108.

[BS93] D. Buchrolz, S. J. Summers. An Algebraic Characterization of Vacuum States in Minkowski Space. Commun. Math. Phys. 155 (1993) 449-458.

[BS03] A. N. Bernal, M. SÁnchez. On Smooth Cauchy Hypersurfaces and Geroch's Splitting Theorem. Commun. Math. Phys. 243 (2003) 461470. arXiv: gr-qc/0306108.

[BS05] - Smoothness of Time Functions and the Metric Splitting of Globally Hyperbolic Spacetimes. Commun. Math. Phys. 257 (2005) 43-50. arXiv: gr-qc/0401112.

[BS06] - Further Results on the Smoothability of Cauchy Hypersurfaces and Cauchy Time Functions. Lett. Math. Phys. 77 (2006) 183-197. arXiv: gr-qc/0512095.

[BS07] -. Globally Hyperbolic Spacetimes can be Defined as "Causal"instead of "Strongly Causal". Clas. Quantum Grav. 24 (2007) 745-749. arXiv: gr-qc/0611138.

[BV95] D. Buchrolz, R. Verch. Scaling Algebras and Renormalization Group in Algebraic Quantum Field Theory. Rev. Math. Phys. 7 (1995) 11951240. arXiv: hep-th/9501063. 
[BW75] J. J. Bisognano, E. H. Wichmann. On the Duality Condition for a Hermitian Scalar Field. J. Math. Phys. 16 (1975) 985-1007.

[BW76] - On the Duality Condition for Quantum Fields. J. Math. Phys. 17 (1976) 303-321.

[BY99] H.-J. Borchers, J. Yngvason. Modular Groups of Quantum Fields in Thermal States. J. Math. Phys. 40 (1999) 601-624. arXiv:math-ph/9805013.

[CK93] D. Christodoulou, S. Klainerman, The Global Nonlinear Stability of the Minkowski Space. Princeton University Press, 1993.

[DH72] J. J. DuistermaAT, L. HÖRmander. Fourier Integral Operators II. Acta Math. 128 (1972) 183-269.

[Die88] J. Dieckmann. Cauchy Surfaces in a Globally Hyperbolic Space-Time. J. Math. Phys. 29 (1988) 578-579.

[DR02] M. Dütsch, K.-H. Rehren. A Comment on the Dual Field in the AdS-CFT Correspondence. Lett. Math. Phys. 62 (2002) 171-184. arXiv: hep-th/0204123.

[DR03] - Generalized Free Fields and the AdS-CFT Correspondence. Ann. Henri Poincaré 4 (2003) 613-635. arXiv:math-ph/0209035.

[Dri77] W. Driessler. On the Type of Local Algebras in Quantum Field Theory. Commun. Math. Phys. 53 (1977) 295-297.

[Dug66] J. Dugundji, Topology. Allyn and Bacon, 1966.

[Dui96] J. J. DuistermaAt, Fourier Integral Operators, Progress in Mathematics, vol. 130. Birkhäuser, 1996.

[EG92] L. C. Evans, R. F. GARIEPy, Measure Theory and Fine Properties of Functions. CRC/Longman Press, 1992.

[FG85] C. Fefferman, C. R. Graham, Conformal Invariants. In: Élie Cartan et les mathématiques d'aujourd'hui, Astérisque, vol. hors série, Societé Mathématique de France, Lyon, 1985, 95-116.

[FH81] K. Fredenhagen, J. Hertel. Local Algebras of Observables and Pointlike Localized Fields. Commun. Math. Phys. 80 (1981) 555-561.

[FH90] K. Fredenhagen, R. HaAG. On the Derivation of the Hawking Radition Associated with the Formation of a Black Hole. Commun. Math. Phys. 127 (1990) 273.

[Fri75] F. G. Friedlander, The Wave Equation on a Curved Space-Time. Cambridge University Press, 1975. 
[Gal00] G. J. Galloway. Maximum Principles for Null Hypersurfaces and Null Splitting Theorems. Ann. Henri Poincaré 1 (2000) 543-567. arXiv:math.DG/9909158.

[Ger68] R. Geroch. What is a Singularity in General Relativity? Ann. Phys. (N.Y.) 48 (1968) 526-540.

[Ger70] —. Domain of Dependence. J. Math. Phys. 11 (1970) 437-449.

[GH81] M. J. Greenberg, J. R. HARPer, Algebraic Topology - A First Course. Addison-Wesley, 1981.

[GKP72] R. Geroch, E. H. Kronheimer, R. Penrose. Ideal Points in SpaceTime. Proc. Roy. Soc. Lond. A 327 (1972) 545-567.

[GKP98] S. S. Gubser, I. R. Klebanov, A. M. Polyakov. Gauge Theory Correlators from Non-Critical String Theory. Phys. Lett. B 428 (1998) 105-114. arXiv:hep-th/9802109.

[GL91] C. R. Graham, J. M. Lee. Einstein Metrics with Prescribed Conformal Infinity on the Ball. Adv. Math. 87 (1991) 186-225.

[GLRV01] D. Guido, R. Longo, J. E. Roberts, R. Verch. Charged Sectors, Spin and Statistics in Quantum Field Theory on Curved Spacetimes. Rev. Math. Phys. 13 (2001) 125-198. arXiv:math-ph/9906019.

[GN43] I. M. Gel'Fand, M. A. NAimark. On the Imbedding of Normed Rings into the Ring of Operators in Hilbert Space. Mat. Sb. 12 (1943) 197213.

[Gra00] C. R. Graham. Volume and Area Renormalizations for Conformally Compact Einstein Metrics. Rend. Circ. Mat. Palermo Ser. II Suppl. 63 (2000) 31-42. arXiv:math.DG/9909042.

[GS07] G. W. Gibbons, S. N. Solodukhin. The Geometry of Small Causal Diamonds. Phys. Lett. B 649 (2007) 317-324. arXiv: hep-th/0703098.

[GSWW99] G. Galloway, K. Schleich, D. M. Witt, E. Woolgar. Topological Censorship and Higher Genus Black Holes. Phys. Rev. D 60 (1999) 104.039. arXiv: gr-qc/9902061.

[GT98] D. Gilbarg, N. S. Trudinger, Elliptic Partial Differential Equations of Second Order. 2nd. ed., Springer-Verlag, 1998.

[GW00] S. GAO, R. M. WALD. Theorems on Gravitational Time Delay and Related Issues. Clas. Quantum Grav. 17 (2000) 4999-5008. arXiv: gr-qc/0007021.

[Haa96] R. HAag, Local Quantum Physics - Fields, Particles, Algebras. 2nd. ed., Springer-Verlag, 1996. 
[Had03] J. Hadamard, Lectures on Cauchy's Problem in Linear Partial Differential Equations. Dover, 1923, 1952, 2003.

[Haw71] S. W. Hawking. Gravitational Radiation from Colliding Black Holes. Phys. Rev. Lett. 26 (1971) 1344-1346.

[Haw75] -. Particle Creation by Black Holes. Commun. Math. Phys. 43 (1975) 199-220.

[HE73] S. W. Hawking, G. F. R. Ellis, The Large Scale Structure of SpaceTime. Cambridge University Press, 1973.

[HHo0] G. T. Horowitz, V. E. Hubeny. Quasinormal Modes of AdS Black Holes and the Approach to Thermal Equilibrium. Phys. Rev. D 62 (2000) 024.027. arXiv:hep-th/9909056.

[HHW67] R. HaAg, N. M. Hugenholtz, M. Winnink. On the Equilibrium States in Quantum Statistical Mechanics. Commun. Math. Phys. 5 (1967) $215-236$.

[Him05] S. Hollands, A. Ishibashi, D. Marolf. Comparison between Various Notions of Conserved Charges in Asymptotically AdS-spacetimes. Clas. Quantum Grav. 22 (2005) 2881-2920. arXiv:hep-th/0503045.

[Hir76] M. W. Hirsch, Differential Topology. Springer-Verlag, 1976.

[HK64] R. HAAG, D. KAstler. An Algebraic Approach to Quantum Field Theory. J. Math. Phys. 5 (1964) 848-861.

[Hoo93] G. 'T Hooft, Dimensional Reduction in Quantum Gravity. In: SalamFestschrift (A. Ali ET AL., ed.), World Scientific, 1993, 284-296. arXiv: gr-qc/9310026.

[Hör71] L. HöRmander. Fourier Integral Operators I. Acta Math. 127 (1971) 79-183.

[Hör90] - The Analysis of Linear Partial Differential Operators I - Distribution Theory and Fourier Analysis. 2nd. ed., Springer-Verlag, 1990.

[HS98] M. Henningson, K. Skenderis. The Holographic Weyl Anomaly. JHEP 9807 (1998) 023. arXiv:hep-th/9806087.

[HS00] - Holography and the Weyl Anomaly. Fortsch. Phys. 48 (2000) 125-128. arXiv: hep-th/9812032.

[HSS01] S. de Haro, K. Skenderis, S. Solodukhin. Holographic Reconstruction of Spacetime and Renormalization in the AdS/CFT Correspondence. Commun. Math. Phys. 217 (2001) 595-622. arXiv: hep-th/0002230. 
[Ino98] A. Inoue, Tomita-Takesaki Theory in Algebras of Unbounded Operators, Lecture Notes in Mathematics, vol. 1699. Springer-Verlag, 1998.

[Isr86] W. IsRAel. Third Law of Black Hole Dynamics: A Formulation and Proof. Phys. Rev. Lett. 57 (1986) 397-399.

[Jac85] N. Jacobson, Basic Algebra I. 2nd. ed., W. H. Freeman Co., 1985.

[Joh82] F. John, Partial Differential Equations. 4th. ed., Springer-Verlag, 1982.

[Kad00] L. P. Kadanoff, Statistical Physics - Statics, Dynamics and Renormalization. World Scientific, 2000.

[KF75] A. N. Kolmogorov, S. V. Fomin, Introductory Real Analysis (Translation by R. A. Silverman). Dover, 1975.

[Kic04] S. Kichenassamy. On a Conjecture of Fefferman and Graham. Adv. Math. 184 (2004) 268-288.

[KR86] R. V. Kadison, J. R. Ringrose, Fundamentals of the Theory of Operator Algebras - vol. 2 (Advanced Theory). Academic Press, 1986.

[Lan69] L. J. Landau. A Note on Extended Locality. Commun. Math. Phys. (1969) 246-253.

[Lan98] S. MAC LANE, Categories for the Working Mathematician. 2nd. ed., Springer-Verlag, 1998.

[Lan99] S. Lang, Complex Analysis. 4th. ed., Springer-Verlag, 1999.

[Lie67a] E. H. LiEB. Exact Solution of the Problem of the Entropy of TwoDimensional Ice. Phys. Rev. Lett. 18 (1967) 692-694.

[Lie67b] - Residual Entropy of Square Ice. Phys. Rev. 162 (1967) 162-172.

[LM75] M. LÜscher, G. MACK. Global Conformal Invariance in Quantum Field Theory. Commun. Math. Phys. 41 (1975) 203-234.

[Mal98] J. Maldacena. The Large N Limit of Superconformal Field Theories and Supergravity. Adv. Theor. Math. Phys. 2 (1998) 231-252. arXiv: hep-th/9711200.

[Min00] R. A. Minlos, Introduction to Mathematical Statistical Mechanics, University Lecture Series, vol. 19. American Mathematical Society, 2000.

[Mr03] P. Martinetti, C. Rovelli. Diamond's Temperature: Unruh Effect for Bounded Trajectories and Thermal Time Hypothesis. Clas. Quantum Grav. 20 (2003) 4919-4931.

[Müg96] M. MÜGER, Disorder Operators, Quantum Doubles, and Haag Duality in 1+1 Dimensions (1996). Seminar given at NATO ASI "Quantum Fields and Quantum Spacetime", Cargèse, arXiv:hep-th/9611131. 
[Müg98] -. Quantum Double Actions on Operator Algebras and Orbifold Quantum Field Theories. Commun. Math. Phys. 191 (1998) 137-181. arXiv: hep-th/9606175.

[Mun71] M. E. Munroe, Measure and Integration. 2nd. ed., Addison-Wesley, 1971.

[Mur90] G. J. Murphy, C*-algebras and Operator Theory. Academic Press, 1990.

[O’N83] B. O’Neill, Semi-Riemannian Geometry. Academic Press, 1983.

[OP93] M. OhyA, D. Petz, Quantum Entropy and its Use. Springer-Verlag, 1993.

[PSW93] R. Penrose, R. D. Sorkin, E. Woolgar, A Positive Mass Theorem Based on the Focusing and Retardation of Null Geodesics (1993). Preprint, arXiv:gr-qc/9301015.

[PSW02] D. N. PAge, S. Surya, E. Woolgar. Positive Mass from Holographic Causality. Phys. Rev. Lett. 89 (2002) 121.301. arXiv: hep-th/0204198.

[Reh00] K.-H. Rehren. Algebraic Holography. Ann. Henri Poincaré 1 (2000) 607623. arXiv: hep-th/9905179.

[Reh05] -, QFT Lectures on AdS-CFT. In: Proceedings of the 3rd Summer School in Modern Mathematical Physics, Institue of Physics, Belgrade, 2005, 95-118. arXiv: hep-th/0411086.

[Rib04] P. L. Ribeiro, Renormalization Group Flow in Algebraic Holography. In: Proceedings of the International Conference on Renormalization Group and Anomalies in Gravity and Cosmology (M. Asorey, I. L. Shapiro, eds.), Nucl. Phys. B (Proc. Suppl.), vol. 127, Ouro Preto, Brazil, 2004, 193-195. arXiv:hep-th/0306024.

[Rib07] -, Algebraic Holography in Asymptotically Simple, Asymptotically AdS Spacetimes. In: Rigorous Quantum Field Theory - a Festschrift in the Honour of Jacques Bros (A. Boutet DE Monvel, D. BuChHolz, D. Iagolnitzer, U. Moschella, eds.), Progress in Mathematics, vol. 251, Birkhäuser, SPhT-CEA Saclay, Paris, 2007, 253-270. arXiv:hep-th/0502096.

[Rob04] J. E. RoberTs, More Lectures in Algebraic Quantum Field Theory. In: Noncommutative Geometry - C.I.M.E. Lectures (S. DopliCHER, R. Longo, eds.), Lecture Notes in Mathematics, vol. 1831, SpringerVerlag, Martina Franca, Italy, 2004, 263-342.

[RS75] M. ReEd, B. Simon, Methods of Modern Mathematical Physics II - Fourier Analysis, Self-Adjointness. Academic Press, 1975.

[RS80] - Methods of Modern Mathematical Physics I - Functional Analysis. Revised ed., Academic Press, 1980. 
[Rud87] W. Rudin, Real and Complex Analysis. 3rd. ed., McGraw-Hill, 1987.

[Rud91] —, Functional Analysis. 2nd. ed., McGraw-Hill, 1991.

[Sch96] D. Schlingemann. On the Algebraic Theory of Soliton and Antisoliton Sectors. Rev. Math. Phys. 8 (1996) 301-326. arXiv:hep-th/9601015.

[Sch05] B. Schroer, Area Density of Localization-Entropy II: Double ConeHolography, Holographic Symmetry and its Relation to the Classical BMS Group (2005). Preprint, arXiv: hep-th/0511291.

[Sch06] - Area Density of Localization-Entropy I: the Case of WedgeLocalization. Clas. Quantum Grav. 23 (2006) 5227-5248. arXiv:hep-th/0507038.

[Seg47] I. E. Segal. Postulates for General Quantum Mechanics. Ann. Math. 48 (1947) 930-948.

[Sei77] H.-J. SeIfert. Smoothing and Extending Cosmic Time Functions. Gen. Rel. Grav. 8 (1977) 815-831.

[Sew82] G. L. SEwELL. Quantum Fields on Manifolds: PCT and Gravitationally Induced Thermal States. Ann. Phys. (N.Y.) 141 (1982) 201-224.

[Som06] C. Sommer, Algebraische Charakterisierung von Randbedingungen in der Quantenfeldtheorie (2006). Diplomarbeit, Universität Hamburg, http://www.desy.de/uni-th/lqp/psfiles/dipl-sommer.ps.gz.

[Sor79] R. Sorkin. The Quantum Electromagnetic Field in Multiply Connected Space. J. Phys. A: Math. Gen. 12 (1979) 403-421.

[Spi79] M. SPIVAK, A Comprehensive Introduction to Differential Geometry Volume 2. 2nd. ed., Publish or Perish, 1979.

[SS02] D. T. Son, A. O. Starinets. Minkowski-Space Correlators in AdS/CFT Correspondence: Recipe and Applications. JHEP 0209 (2002) 042. arXiv:hep-th/0205051.

[Str00] A. Strohmaier. On the Local Structure of the Klein-Gordon Field on Curved Spacetimes. Lett. Math. Phys. 54 (2000) 249-261. arXiv:math-ph/0008043.

[Sus95] L. Susskind. The World as a Hologram. J. Math. Phys. 36 (1995) 63776396. arXiv:hep-th/9409089.

[SV96] A. Strominger, C. Vafa. Microscopic Origin of the Bekenstein-Hawking Entropy. Phys. Lett. B 379 (1996) 99-104.

[SW00] R. F. Streater, A. S. Wightman, PCT, Spin and Statistics, and All That. Revised ed., Princeton University Press, 2000. 
[Sán05] M. SÁnchez, Causal Hierarchy of Spacetimes, Temporal Functions and Smoothness of Geroch's Splitting. A Revision. In: Proceedings of the XIII School of Differential Geometry - Part II (P. PiCcione, ed.), Matemática Contemporânea, vol. 29, Universidade de São Paulo, São Paulo, Brazil, 2005, 127-155. arXiv:gr-qc/0411143.

[Tak70] M. TAKesAkI, Tomita's Theory of Modular Hilbert Algebras and its Applications, Lecture Notes in Mathematics, vol. 128. Springer-Verlag, 1970 .

[Tak01] - Theory of Operator Algebras I, Encyclopaedia of Mathematical Sciences, vol. 124. Springer-Verlag, 2001.

[Tak03a] - , Theory of Operator Algebras II, Encyclopaedia of Mathematical Sciences, vol. 125. Springer-Verlag, 2003.

[Tak03b] - , Theory of Operator Algebras III, Encyclopaedia of Mathematical Sciences, vol. 127. Springer-Verlag, 2003.

[Tom67] M. Tomita, Quasi-Standard von Neumann Algebras (1967). Preprint.

[Uhl62] A. Uhlmann. Über die Definition der Quantenfelder nach Wightman und Haag. Wiss. Z. Karl-Marx-Univ. Leipzig Math.-Nat. Reihe 11 (1962) 213-217.

[Ver96] R. Verch, Scaling Algebras, the Renormalization Group and the Principle of Local Stability in Algebraic Quantum Field Theory. In: Proceedings of the Conference on Operator Algebras and Quantum Field Theory, Academia dei Lincei, Roma, 1996, 564-577. arXiv: funct-an/9611004.

[Wal80] R. M. WALD. Dynamics in Nonglobally Hyperbolic, Static Space-Times. J. Math. Phys. 21 (1980) 2802-2805.

[Wal84] —, General Relativity. Chicago University Press, 1984.

[Wal94] -, Quantum Field Theory in Curved Spacetime and Black Hole Thermodynamics. Chicago University Press, 1994.

[Wal97] _. "Nernst Theorem"and Black Hole Thermodynamics. Phys. Rev. D 56 (1997) 6467-6474.

[Wei96] S. WeInBerG, The Quantum Theory of Fields II: Modern Applications. Cambridge University Press, 1996.

[Whi34] H. Whitney. Analytic Extension of Differentiable Functions Defined in Closed Sets. Trans. Amer. Math. Soc. 36 (1934) 63-89.

[Wit98] E. Witten. Anti de Sitter Space and Holography. Adv. Theor. Math. Phys. 2 (1998) 253-291. arXiv:hep-th/9802150. 
[Woo94] E. Woolgar. The Positivity of Energy for Asymptotically Antide Sitter Spacetimes. Clas. Quantum Grav. 11 (1994) 1881-1900. arXiv: gr-qc/9404019.

[Zwi04] B. ZwIEBACH, An Introduction to String Theory. Cambridge University Press, 2004. 



\title{
Notas e tradução das epígrafes
}

\author{
i“"Ver o que são as coisas em si, distinguindo matéria, causa, fim." (Tr. Jaime Bruna) \\ ii A tradução literal é "A mim mesmo". Todavia, a tradução latina "oficial" para o título acabou sendo \\ o nome pelo qual estes escritos pessoais, originalmente em grego, do imperador romano tornaram-se \\ mais conhecidos - "Meditações". In: Os Pensadores, terceira edição, editora Abril Cultural, 1985. \\ iii، 'Só ouvimos as perguntas para as quais somos capazes de encontrar resposta." (Tr. Paulo César \\ iv "A Gaia Ciência". Editora Companhia das Letras, 2001.

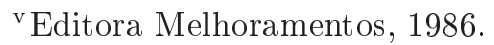 \\ vi
} de Souza)

"Então, já de todo sem juízo, veio a dar com o mais estranho pensamento com que jamais deu algum louco neste mundo, e foi que lhe pareceu conveniente e necessário, tanto para o aumento de sua honra como para o serviço de sua república, fazer-se cavaleiro andante e sair pelo mundo com suas armas e seu cavalo em busca de aventuras e do exercício em tudo aquilo que lera que os cavaleiros andantes se exercitavam, desfazendo todo gênero de agravos e pondo-se em transes e perigos que, vencidos, lhe rendessem eterno nome e fama. Imaginava-se o pobre homem já coroado pelo valor do seu braço, quando menos do império da Trebizonda; e, assim, com tais e tão gratos pensamentos, movido pelo estranho prazer que deles tirava, se deu pressa em levar o seu desejo a termo. E a primeira coisa que fez foi limpar umas armas dos bisavós que, cobertas de ferrugem e azinhavre, longos séculos havia que estavam postas e esquecidas num canto."

(Tr. Sérgio Molina)

vii "O engenhoso fidalgo dom Quixote de la Mancha". Primeira edição bilíngüe, Editora 34, 2002. viii Editora Nova Fronteira, 1988.

ix"Pois sei que o tempo é sempre o tempo / E o espaço é sempre e apenas o espaço / E que o real somente o é dentro de um tempo / E apenas para o espaço que o contém" (Tr. Ivan Junqueira)

x "Quarta-Feira de Cinzas". In: T. S. Eliot-Obra Completa, Volume I-Poesia, edição bilíngüe, editora Arx, 2004.

xi

“- Pelo que eu imagino - disse D. Quixote -, não há história humana no mundo que não tenha os seus altos e baixos, especialmente as que tratam de cavalaria, as quais nunca podem estar plenas de prósperos acontecimentos."

(Tr. Pedro Lauridsen Ribeiro, a partir da edição em espanhol da Real Academia Española (2004))

xiic Ó Deus! Eu poderia estar recluso numa casca de noz, e julgar-me rei do espaço infinito, não fossem os meus sonhos maus." (Tr. Péricles Eugênio da Silva Ramos) xiii "Hamlet, Príncipe da Dinamarca". Editora Abril Cultural, 1976. 
xiv"Há duas maneiras de se alcançar Despina: de navio ou de camelo. A cidade se apresenta de forma diferente para quem chega por terra ou por mar. (...) Cada cidade recebe a forma do deserto a que se opõe; é assim que o cameleiro e o marinheiro vêem Despina, cidade de confim entre dois desertos." (Tr. Diogo Mainardi)

xv “'As cidades e o desejo 3' (As cidades invisiveis)”. Segunda edição, editora Companhia das Letras, 2006

$\mathrm{xvi}$

"- Como pode ser isso? — respondeu D. Quixote. — Tão da essência da história é saber ao certo quantas cabras atravessaram que, errando-se uma do seu número, não podes prosseguir com ela?

- Não, senhor, de maneira alguma - respondeu Sancho -; pois quando eu perguntei a vossa mercê quantas cabras tinham passado, e me respondeu que não sabia, naquele mesmo instante me fugiu da memória o quanto me faltava dizer, e à fé que era de muita virtude e contento.

— De modo - disse D. Quixote - que a história já está acabada?

- Tão acabada como a minha mãe - disse Sancho.

- Digo-te à vera - respondeu D. Quixote - que contaste uma das mais novas fábulas, conto ou história que alguém pôde pensar no mundo, e que tal jeito de contá-la e deixá-la jamais se poderá ver nem terá visto em toda a vida, embora eu não esperasse outra coisa do teu bom discurso; mas isto não me espanta, pois talvez estes golpes que não cessam tenham embotado o teu entendimento."

(Tr. Sérgio Molina)

xvii، Vai, vai, vai, disse o pássaro: o gênero humano / não pode suportar tanta realidade. / O tempo passado e o tempo futuro / O que poderia ter sido e o que foi, / Convergem para um só fim, que é sempre presente." (Tr. Ivan Junqueira)

xviii "Quatro Quartetos". In: T. S. Eliot-Obra Completa, Volume I-Poesia, edição bilíngüe, editora Arx, 2004.

As gravuras nos frontispícios de cada Parte são recortes, feitos pelo Autor, de ilustrações de Gustave Doré para "O engenhoso fidalgo dom Quixote de la Mancha" (Primeiro Livro), de MiGuel De Cervantes SAavedra. As ilustrações, desenhadas originalmente a bico-de-pena, foram gravadas em madeira por HÉLIOdore PisAn, tendo sido empregadas na edição anotada e traduzida para o francês por Louis ViARDOT, publicada pela editora Hachette em 1863 (republicada em 1978). Por terem sido publicadas pela primeira vez há mais de 100 anos, estas ilustrações estão em domínio público, e podem ser encontradas em formato eletrônico na Wikimedia Commons (http://commons.wikimedia.org/wiki/Don_Quixote). 